Portland State University

PDXScholar

Spring 6-4-2019

\title{
Integrating Work Ability into the Organizational Science Literature: Advancing Theory and Developing the Nomological Network
}

Grant Brady

Portland State University

Follow this and additional works at: https://pdxscholar.library.pdx.edu/open_access_etds

Part of the Psychology Commons

Let us know how access to this document benefits you.

\section{Recommended Citation}

Brady, Grant, "Integrating Work Ability into the Organizational Science Literature: Advancing Theory and Developing the Nomological Network" (2019). Dissertations and Theses. Paper 5012.

https://doi.org/10.15760/etd.6888

This Dissertation is brought to you for free and open access. It has been accepted for inclusion in Dissertations and Theses by an authorized administrator of PDXScholar. Please contact us if we can make this document more accessible: pdxscholar@pdx.edu. 
Integrating Work Ability into the Organizational Science Literature:

Advancing Theory and Developing the Nomological Network

by

Grant Brady

A dissertation submitted in partial fulfillment of the requirements for the degree of

\section{Doctor of Philosophy \\ in \\ Applied Psychology}

Dissertation Committee:

Donald M. Truxillo, Chair

Pamela Tierney

David M. Cadiz

Todd Bodner

Portland State University

2019 


\begin{abstract}
As the workforce ages, enabling individuals to work effectively across the lifespan is critical for individuals, organizations, and societies. Put simply, societies and organizations are beginning to face a "new normal" in which people must continue working later in life. Investigations of work ability (WA), an individual's ability to meet the demands of their job, is a line of research that facilitates our understanding of the factors related to working successfully across the lifespan. Although research has established that WA is influenced by a range of organizational and personal factors and linked WA to retirement and disability, a number of gaps and underlying conceptual issues have limited the value of the WA research to the organizational sciences.
\end{abstract}

Through a series of three studies - a meta-analysis (Study 1a) with $k=247$ studies and $N=312,987$ individuals, a supplemental online data collection (Study 1b), a nurse sample (Study 2), and a healthcare sample (Study 3) - this dissertation draws on the JD-R model to move the WA literature forward and advance WA theory within the organizational science literature. Study 1 provides a quantitative synthesis of the WA literature, establishes its nomological network, and provides a straightforward conceptual definition of WA. This synthesis provides a roadmap for researchers and practitioners by highlighting leverage points to promote WA across the lifespan. Second, these studies answer lingering questions regarding the concept of WA. In doing so, these studies provide a clear conceptual distinction between WA as measured by the Work Ability Index (WAI), which includes health-based questions, and measures of perceived WA (PWA), which are perceptions of WA as rated by individual. PWA measures performed 
similarly to the WAI in the vast majority of analyses, lending substantial support to the use of relatively simple PWA measures. Third, Study 1 and Study 2 provide evidence that PWA explains unique variance in organizational (e.g., engagement, burnout) and personal (e.g., disability intentions, health) correlates above and beyond the established constructs of perceived fit, general self-efficacy, and job self-efficacy. Fourth, Study 2 identified age discrimination as an important yet understudied antecedent of WA and showed that PWA can serve as a mediator between age discrimination and negative outcomes (e.g., lower life satisfaction and task performance). Finally, Study 3 showed that PWA is related to supervisor ratings of task and creative performance. Taken together, these three studies situate WA within the organizational literature and provide substantial evidence of the value of WA for meeting the challenges of an aging and agediverse workforce. 


\section{Table of Contents}

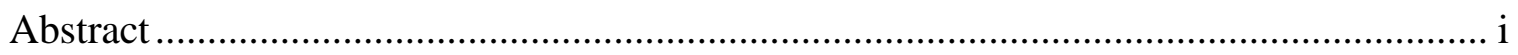

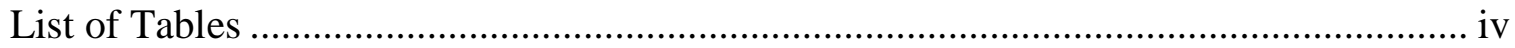

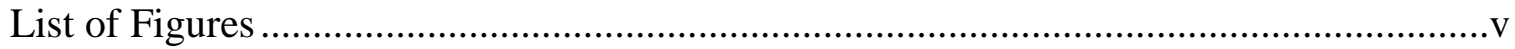

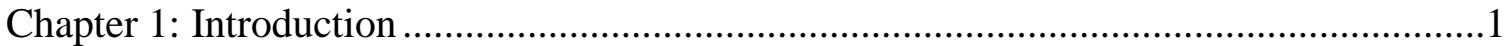

Chapter 2: Opening the Black Box: Examining the Nomological Network of Work Ability and Its Role in Organizational Research ....................................................12

Chapter 3: Age Discrimination and Job Outcomes: The Mediating Role of Perceived

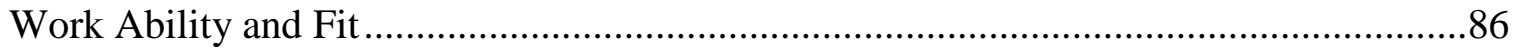

Chapter 4: Linking Perceived Work Ability to Supervisor-Rated Job Performance.......123

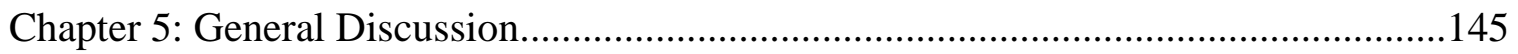

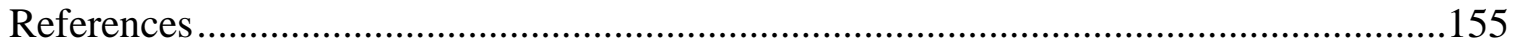

Appendix Study Level Results from Meta-Analysis ..................................................209 


\section{List of Tables}

Table 1 Random-Effects Meta-Analytic Estimates of the Relationships between Work Ability and Its Theoretical Antecedents.

Table 2 Random-Effects Meta-Analytic Estimates of the Relationships between Work Ability and its Theoretical Outcomes.............................................74

Table 3 Mixed-Effects Meta-Analytic Estimates of the Relationships Between WA and its Antecedents (by WA Measure) .............................................. 75

Table 4 Mixed-Effects Meta-Analytic Estimates of the Relationships Between WA and its Outcomes (by WA Measure) ............................................78

Table 5 Mixed-Effects Meta-Analytic Estimates of the Relationships Between WA and its Antecedents and Outcomes (by Occupation)...................................79

Table 6 Mixed-Effects Meta-Analytic Estimates of the Relationships Between WA and

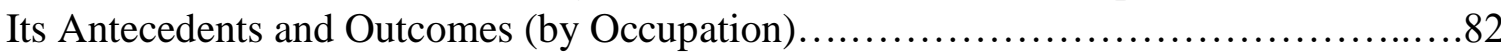

Table 7 Zero-Order Correlations among Study 2 Variables.............................83

Table 8 Sequential Multiple Regression Models Examining Incremental Variance Explained by Perceived WA in Relation to Correlates over and above Perceived Fit, General Self-Efficacy, and Job Self-Efficacy.................................. 85

Table 9 Study 2 Sample Descriptive Statistics.................................120

Table 10 Means, Standard Deviations, and Zero-Order correlations among Study 2 Variables

Table 11 Means, Standard Deviations, and Zero Order Correlations among Study 3 Variables. 


\section{List of Figures}

Figure 1 Theoretical Model of Work Ability.................................... 70

Figure 2 Results Study 1 A................................................ 71

Figure 3 Study 2 Conceptual Model...........................................119

Figure 4 Study 2 Results.............................................. 121

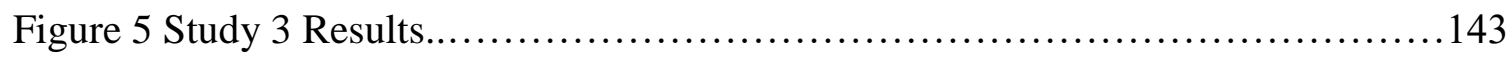

Figure 6 Study 3 Moderation Plot............................................ 144 


\section{Chapter 1: Introduction}

Older workers are a growing portion of the population, and this trend is expected to continue for the foreseeable future (Toossi, 2015; U.S. Census Bureau, 2019). As the workforce continues to age, workforce participation is also expected to continue decreasing to an estimated $61 \%$ by 2026 in the United States (U.S. Bureau of Labor Statistics, 2017). This is problematic because retirement systems and other social safety programs rely on funds from the active workforce. However, lower workforce participation likely means fewer available funds, but a larger pool of people pulling from those funds. Additionally, the loss of human capital may cause labor shortages and cause organizations to lose important institutional knowledge (e.g. "the brain drain"; Docquier \& Rapoport, 2012; Hawkes, Kolenko, Hockness, \& Diwaker, 2009). This is increasingly a concern for organizations in general (Kulik, Ryan, Harper, \& George, 2014) and among specific industries, such as nursing, where the workforce is aging, but demand for those roles is increasing as societies age and require more healthcare services (Eley et al., 2007; Sherman, Chiang-Hanisko, \& Koszalinski, 2013).

In order to address this demographic shift, two factors are becoming increasingly important to consider: First, identifying methods to promote working later in life (Kulik et al., 2014; Truxillo, Cadiz, \& Hammer, 2015) and second, gaining a better understanding of how workers are treated across the lifespan (Armstrong-Stassen \& Schlosser, 2008; Bilinska, Wegge, \& Kliegel, 2016; Fisher, Truxillo, Finkelstein, \& Wallace, 2017). One line of research aimed at better understanding how to retain workers and extend working careers is research on work ability (WA) and perceived WA (PWA; 
Feldt, Hyvönen, Mäkikangas, Kinnunen, \& Kokko, 2009; Ilmarinen et al., 1991, 1997, McGonagle, Fisher, Barnes-Farrell, \& Grosch, 2015). This research has shown that WA can predict important outcomes such as exit from the workforce via retirement and disability later in life (von Bonsdorff et al., 2011). More recently, organizational researchers have found that a range of psychosocial variables influence WA, particularly when measured as PWA (e.g., social support, job demands, personal resources; McGonagle et al., 2015; Palermo, Fuller-Tyszkiewicz, Walker, Appannah, 2013). Although older workers are an increasing portion of the US population (Toossi, 2015; U.S. Census Bureau, 2017), negative stereotypes about older workers persist (Cuddy, Norton, \& Fiske, 2005; Posthuma \& Campion, 2008) and occur both implicitly and explicitly (Nosek et al., 2007). These stereotypes include being low in competence (Cuddy et al., 2005; Fiske, Cuddy, Glick, \& Xu, 2002), less willing to engage in trainings (Ng \& Feldman, 2012), unable to learn new material, and more likely to turnover (Posthuma \& Campion, 2009). Further, age stereotypes have been shown to exist acrosscultures (Chiu, Chan, Snape, \& Redman, 2001) and are viewed as more culturally acceptable than other forms of bias (e.g., racism, sexism; Levy \& Banaji, 2002). Based on evidence that age discrimination occurs and the need to better understand how to extend working careers, it is important to understand how the treatment of older workers, specifically age discrimination or the lack thereof, influences WA and organizational outcomes.

Work Ability, Its Antecedents, and Its Outcomes

Before delving into the theoretical background of WA, it is important to understand the development of the WA construct itself. As a concept, WA was formally 
developed in Finland in the early 1980s to help understand how long individuals could be expected to continue working (Ilmarinen et al., 1991, 1997). In their initial series of studies, Ilmarinen and colleagues $(1991,1997)$ found that WA could predict actual exit behaviors including disability pension status and retirement years after the initial measurement of WA. These studies also identified a number of factors associated with increased risk for poor WA. Specifically, they found that those working in blue-collar occupations and those 55 years old or older tended to experience greater declines in WA compared to white-collar and younger workers (Ilmarinen et al., 1991, 1997).

Since the initial research, WA research has expanded dramatically and the most common measure of WA, the Work Ability Index (WAI), is now available in over 26 languages (Ilmarinen, 2009; Morschhäuser \& Sochert, 2007). Researchers have also identified a wide range of individual (e.g., personal health and core self-evaluations; McGonagle et al., 2015; Palermo et al., 2015) and organizational factors that positively (e.g., autonomy; Weigly et al., 2013) or negatively (e.g., physical job demands; von Bonsdorff, 2012) influence WA. Further, researchers have demonstrated the utility of WA measures for identifying those in need of rehabilitation services (Bethge, Radoschewski, \& Gutenbrunner, 2012b) in addition to longer term outcomes (e.g., retirement, disability status). Taken together, WA clearly has great potential for individuals, organizations, and societies in terms of being able to predict important longterm outcomes, and thus, WA has the potential to serve as a leverage point to promote working successfully across the lifespan. 
Although this research has been informative, a number of serious gaps remain in the extent WA literature. First, despite its widespread use, no studies have quantitatively synthesized the antecedents and outcomes of WA in a comprehensive manner. Second, as the WA literature has developed, the way WA is measured has shifted in fundamental ways, yet the WA literature has not sufficiently investigated how these different types of measures influence the predictive utility of WA. Third, although WA is often studied in the context of the aging workforce and age has been identified as a risk factor for poor WA, researchers have not investigated how perceived age discrimination against an individual relates to WA. Finally, although WA has been linked to self-rated performance and company level performance ratings by the CEO, the link between WA and supervisor ratings of individual performance has not been investigated. Given that job performance is often viewed as one of the most important outcomes in organizational research, this represents a key gap in the WA literature.

Through a series of three studies, I will address these issues by drawing on the job demands-resources (JD-R) model (Bakker \& Demerouti, 2007; Demerouti et al., 2001) and organizational justice theory (Gilliland, 1993; Greenberg, 1987, 1990) to better understand the antecedents and outcomes of WA, how WA is influenced by age discrimination, and how age discrimination and WA work together to influence job outcomes. Specifically, Study 1a provides a meta-analysis of the theoretical antecedents and outcomes of WA and assesses the type of WA measure and occupation as moderators to these relations. In addition, Study1b evaluates the extent to which PWA can explain unique variance in outcomes of health and organizational factors when considering 
established self-perception constructs. Study 2, extends the findings from Study 1 to link age discrimination to WA and empirically test WA as a mediator between age discrimination and retirement intentions, profession turnover intentions, and self-report job performance. Finally, Study 3 tests the relationship between WA and supervisor ratings of engagement and two facets of job performance.

Theoretical Frameworks for Understanding the Role of WA

To better understand the antecedents and outcomes of WA several theories can be considered. First, the JD-R model developed be Demerouti and colleagues (2001) is a resource based theory which posits that strain and negative workplace outcomes develop when high levels of demands are required to accomplish job tasks. One alternative theory that could be considered is conservation of resources (COR) theory (Hobfoll, 1989). Conservation of resources theory posits that under conditions of low resources, individuals seek to maintain their existing resources whereas individual seek out new resources when their resource levels are high. Given that the definition of WA is, "one's ability to meet the demands of their job" and that perceived WA (PWA) is referred to as "perceptions of one's ability to meet the demands of their job," as well as previous literature drawing on the JD-R model to understand WA (Cadiz, Brady, Truxillo, \& Rineer, 2018; McGonagle et al., 2015), I use the JD-R model to guide hypothesis development regarding WA and PWA. In addition to the JD-R model, I supplement the JD-R model with a second theory that focuses specifically on fair treatment, organizational justice theory (Gilliland, 1993; Greenberg, 1987, 1990), to better explain the relation between age discrimination and PWA. Organizational justice theory includes three primary dimensions, and suggests that unfair treatment in terms of organizational 
procedures, allocation of resources, or interpersonal treatment will have negative consequences for organizational outcomes including performance, attitudes, and retention.

Job Demands-Resources Model

In order to understand the antecedents and outcomes of WA, researchers have begun to use the JD-R model (Bakker \& Demerouti, 2007; Demerouti et al., 2001) as a theoretical framework (Cadiz et al., 2018; McGonagle et al., 2015). The JD-R model makes several important propositions. First, it posits that characteristics of a job can generally be defined as either a job resource or a job demand. Job resources refer to aspects of the job that provide growth opportunities or are helpful in accomplishing work goals. On the other hand, job demands are aspects of the job that require sustained effort or skill and come at psychological or physiological costs (Bakker \& Demerouti, 2007; Demerouti et al., 2001).

Early research using the JD-R model focused primarily on burnout and subsequently on engagement (Bakker \& Demerouti, 2007; Demerouti et al., 2001). However, since its original conceptualization, the JD-R model has been extended in a number of important ways to more thoroughly capture the psychosocial processes occurring in the workplace. First, job demands have been specified as either challenge or hindrance demands (Crawford, LePine, \& Rich, 2010) in which both demands may negatively influence wellbeing, but challenge demands may also serve a motivational purpose. In the case of age discrimination, the unfair treatment an individual receives based on their age would likely be perceived as a hindrance demand. Second, the JD-R 
model has been extended to include a health impairment process, rather than exclusively focusing on burnout, as leading to negative organizational outcomes (Bakker \& Demerouti, 2007; Demerouti \& Bakker, 2011). Third, the JD-R model has been expanded to include a motivational process, as opposed to focusing on engagement exclusively, which leads to positive organizational outcomes (Bakker \& Demerouti, 2007; Demerouti \& Bakker, 2011). In addition, the JD-R model has been expanded to account for personal resources (e.g., core self-evaluations) as factors that may influence the two primary pathways (Bakker \& Demerouti, 2007; Demerouti \& Bakker, 2011; Xanthopoulo et al., 2007).

The JD-R model is also well suited to understanding the concept of WA, including its antecedents and outcomes. As noted above, WA is defined as one's ability to meet the demands of their job (Ilmarinen et al., 1991). Similarly, the JD-R model accounts for job demands as well as job and personal resources that facilitate meeting the demands on the job. Thus, I argue that WA can be viewed as another mediating mechanism along the health impairment pathway included in the JD-R whereby job and personal resources positively influence WA and job demands negatively influence WA. This approach has recently by adopted in both empirical research (McGonagle et al., 2015; Palermo et al., 2013) as well as a qualitative overview of the WA literature (Cadiz et al., 2018).

Organizational Justice Theory

Although the JD-R model is well suited to understand the antecedents and outcomes of WA, and age discrimination can be considered a hindrance demand within the JD-R framework, age discrimination can also be view through other theoretical 
lenses. One theory that highlights the importance of considering how individuals are treated is organizational justice theory (Gilliland, 1993; Greenberg, 1987, 1990).

Organizational justice theory includes three primary dimensions of justice and metaanalytic research has shown that each of the dimensions are related to important workrelated outcomes (e.g., withdrawal, job satisfaction, job performance; Colquitt, Conlon, Wesson, Porter, \& Ng, 2001; Colquitt et al., 2013). Within the organizational justice framework, the three dimensions of justice are: procedural justice, defined as fairness in the procedures for distributing resources and making decisions (Gilliland, 1993), distributive justice, defined as fairness in the actual allocation of resources or decision outcomes (Gilliland, 1993), and interactional justice, or the fair interpersonal treatment of an individual within organizational functions (Bies \& Moag, 1986). According to organizational justice theory, violation of any of these three types of justice can lead to negative consequences. For example, violations of justice have been linked to greater withdrawal and negative reactions, whereas greater levels of justice have been linked to performance and more positive job attitudes (Colquitt et al., 2001, 2013).

Although the distinction between each aspect of justice is important, the overarching principle behind each of these dimensions is the perception of fair treatment. This commonality is demonstrated in that each of these dimensions generally relate to outcomes of interest in the same direction, but with varying magnitudes (e.g., Colquitt, 2001, 2013). As I will argue in Study 2, that overarching principle of fair treatment can be applied to age discrimination, which can be viewed as a form of unfair treatment. Specifically, age discrimination can be viewed as unfair treatment that is further specified 
as being due to one's age, and therefore, age discrimination should predict poor work outcomes in a similar pattern compared to forms of injustice that are defined more broadly.

Taken together, I argue that organizational justice theory can serve as a theoretical lens to understand how age discrimination influences work outcomes, and that age discrimination is an increasingly important factor to consider given the demographic shift to an aging and more age-diverse workforce. Tying age discrimination to WA, research has linked WA to other forms of fair treatment, such a balance between one's perceived effort and rewards they receive from their job (Bethge, Radoschewski, \& Gutenbrunner, 2012a). However, to date, the relationship between age discrimination and WA, and how age discrimination and PWA work together to influence work outcomes remains understudied.

\section{Present Studies}

Together, the three studies that makeup this dissertation provide a better understanding the antecedents and outcomes of WA, as well as how age discrimination and WA operate to influence work outcomes. These studies contribute to the literature by addressing several of the most pressing questions and gaps within the WA literature. To accomplish this, I draw on the JD-R model and organizational justice theory as lenses to make theoretically grounded hypotheses about how these variables influence one another. Given the aging and increasingly age-diverse workforce, these questions are both relevant for organizations and increasingly timely to consider.

Study 1 provides a comprehensive and multi-disciplinary meta-analysis of the extent literature. This search spans across the organizational psychology and medical 
literatures to assess the theoretical antecedents and outcomes of WA. A quantitative synthesis of this literature is critical to better understand the complete scope of variables that influence, and are influenced by, WA. In addition, Study 1 addresses a key question in the WA literature, that is, does the way in which WA is measured influence the relationships between WA and its antecedents and outcomes? Further, Study 1 evaluates occupation category as a potential moderator of these relations, another important question. Finally, Study 1 provides initial evidence that PWA can explain incremental variance in organizational outcomes, beyond perceived fit and job self-efficacy.

Study 2 extends beyond Study 1 to assess an additional potential antecedent of WA, age discrimination, and assess WA as a mediating mechanism between age discrimination and job outcomes. This process is implied, but not tested in Study 1. In addition to assessing this mediating process, Study 2 will also examine the extent to which WA remains a significant mediator between age discrimination and organizational outcomes when simultaneously considering perceived fit as a mediating mechanism. This step is critically important to better understand the relative value of WA compared to its related constructs.

Finally, Study 3 addresses an additional important gap in the WA literature. That is, although WA has been linked to exit from the workforce, it has not been linked supervisor ratings of job performance. WA has been linked to self-report ratings of performance as well as company level ratings of performance. However, those ratings are generally not used by organizations to make employee-based decisions and do not address specific facets of job performance. More specifically, studies examining the 
relationship between PWA and self-rated performance introduce inflation because one's perceptions about their ability to meet the demands of their job is likely tied to their belief about their job performance, which makes it difficult to understand the true PWA performance relationship. Given the noted limitations of research on WA and performance, Study 3 expands on existing research to more firmly link PWA to facets of performance, and evaluates age as a moderator to these relations. 
Chapter 2: Opening the Black Box: Examining the Nomological Network of Work Ability and Its Role in Organizational Research

\author{
Grant Brady, Donald M. Truxillo, and David M. Cadiz \\ Portland State University \\ Jennifer R. Rineer \\ RTI International \\ David E. Caughlin and Todd Bodner \\ Portland State University
}

(First round revision sent to: Journal of Applied Psychology)

Author Note

Grant Brady, Department of Psychology, Portland State University; Donald M. Truxillo, Department of Psychology, Portland State University; David M. Cadiz, School of Business, Portland State University; Jennifer R. Rineer, RTI International; David Caughlin, School of Business, Portland State University; Todd Bodner, Department of Psychology, Portland State University. A special thank you to Cosimo Gonnelli and Aaron Greenfield for their assistance with coding for this study. Portions of this research were supported by the National Institute of Occupational Safety and Health Grant T03OH008435 awarded to Portland State University. 


\begin{abstract}
The aging of the industrialized workforce has spurred research on how to support people working later in life. Within this context, the concept of work ability, or an employee's ability to meet their job requirements, has been introduced as an explanatory mechanism for understanding employee disability, wellbeing, attitudes, and behavior. However, the work ability concept has evolved across disparate literatures with multiple, contentdiverse measures and often with little consideration of theory or examination of its nomological network. Using the job demands-resources model as a framework, we present a meta-analytic summary $(k=247 ; N=312,987)$ of work ability's correlates and potential moderators of these relationships. Taken together, we found consistent negative relationships between job demands and work ability, and consistent positive relationships between job and personal resources and work ability. Work ability was also associated with important job outcomes including job attitudes and actual behaviors such as absenteeism and retirement. Measures of work ability that include both perceived and objective components generally showed stronger relationships than did exclusively perceptual measures, and occupation type was a significant moderator of certain relations between work ability and its correlates. We supplemented this meta-analysis with a primary data collection to examine differences between perceived work ability and the conceptually similar variables of self-efficacy and perceived fit, demonstrating that perceived work ability can explain incremental variance in job- and health-related variables. Our discussion focuses on the value of the work ability construct for both research and practice and future directions for work ability research.
\end{abstract}


Opening the Black Box: Examining the Nomological Network of Work Ability and Its Role in Organizational Research

As the industrialized workforce continues to age, an estimated $38.2 \%$ of the workforce in the United States will be at least 55 years old by 2024 (Toossi et al., 2015). This trend is a driving factor in the expected decline of the overall labor force participation rate in the United States (61\% by 2026; U.S. Bureau of Labor Statistics, 2017). The aging workforce and low rates of workforce participation place increased strain on retirement systems and other social safety net programs. To address the challenges associated with an aging workforce and lower workforce participation, it is critical to understand how to keep individuals working effectively and participating in the labor market across the lifespan. Continuing to work can also provide individuals with important benefits, such as financial (Butrica, 2011), psychological (Kulik, Ryan, Harper, \& George, 2014; Rohwedder \& Willis, 2010; Zhan, Wang, Liu, \& Shultz, 2009), and health benefits (Herzog, House, \& Morgan, 1991). In addition, organizations can benefit from older employees' positive job attitudes (Ng \& Feldman, 2008), depth of job knowledge, and higher rates of organizational citizenship behaviors (Ng \& Feldman, 2008).

Research on work ability focuses on extending working careers and promoting workforce participation. As a construct, work ability evolved in the field of occupational medicine. Work ability (WA) was originally defined as a person's physical and mental ability to meet the demands of their job (Ilmarinen, 2009; Ilmarinen, et al., 1991a). Its measurement was empirically (not theoretically) derived, but the construct has now been 
adopted by the organizational psychology literature (e.g., McGonagle, Fisher, BarnesFarrell, \& Grosch, 2015; Palermo, Fuller-Tyszkiewicz, Walker, Appannah, 2013).

Research has shown that WA is related to key employment outcomes such as retirement and future disability status (Feldt, Hyvönen, Mäkikangas, Kinnunen, \& Kokko, 2009; Tuomi, Vanhala, Nykyri \& Janhonen, 2004). Moreover, WA has been linked to important job attitudes (e.g., organizational commitment; Feldt et al., 2009), job demands (e.g., role overload; McGonagle, et al., 2015), job resources (e.g., supervisor support; Sugimura \& Thériault, 2010), and personal resources (e.g., self-efficacy, health, and coping; Palermo et al., 2013). Given the links between WA and these work-related factors, WA research is well-positioned to inform organizational practices and interventions that support individuals working effectively throughout their careers.

Although the concept of WA can influence research and practice on supporting employees across the lifespan, several questions remain regarding the WA construct. First, despite the WA literature being sufficiently mature, a quantitative synthesis of its nomological network has not been conducted. Given the multidisciplinary nature of the WA construct, this is particularly important for bridging WA research across disciplines. Second, although WA varies across occupations, there has yet to be a systematic assessment of how occupation influences the relationships between WA and its correlates. Third, although WA is clearly useful as a predictive tool, only one study has investigated the convergent and divergent validity of WA (McGonagle et al., 2015), and none to our knowledge have investigated if WA can explain unique variance in important job and health correlates compared to established constructs in the organizational 
sciences such as perceived fit, general self-efficacy, and job self-efficacy. Finally, the multidisciplinary history of WA has in many ways contributed to a rich, broad understanding of its impact on individuals and organizations; on the other hand, it has also resulted in a proliferation of WA measures that vary substantially in terms of the extent to which they include objective (e.g., diagnosed illnesses) or perceived (e.g., selfreport) aspects of WA. ${ }^{1}$ Although measuring a construct via different assessments is not necessarily a concern per se, the highly diverse content of different WA measures warrants further evaluation and is critical for interpreting WA research, identifying research gaps, and moving the WA literature forward.

In the present study, we address these issues by conducting a comprehensive, multidisciplinary (e.g., medicine, psychology), meta-analytic investigation of the WA literature. Using the job demands-resources (JD-R; Bakker \& Demerouti, 2007; Demerouti, Bakker, Nachreiner, \& Schaufeli, 2001) model as a framework, we set out to accomplish the following goals. First, we identify the theoretical antecedents and outcomes of WA across multiple disciplines and assess the strength of these relationships. Second, we evaluate the type of WA measure and occupation type as moderators between WA and its theoretical antecedents and outcomes. In doing so, we shed light on how the content of the WA measure influences the relationships between WA and its theoretical antecedents and outcomes and we discuss the implications of those differences for the utility of the construct. Finally, we examine the relationship

\footnotetext{
${ }^{1}$ When referring to the concept of work ability more broadly, we will use the abbreviation "WA." When referring to perceived work ability specifically, we will use the term "perceived WA." When referring to the Work Ability Index (WAI), which was the original measure of work ability and includes both objective and perceived components, we will use the abbreviation "WAI."
} 
between perceived WA and related constructs (e.g., perceived fit, job self-efficacy) as well as the relative value of WA compared to related constructs in explaining work and health-related factors. Taken together, our goal is to further develop the theoretical underpinnings of the WA construct, the implications of measuring it in different ways and in different contexts, and the implications of WA research for workforce participation and workplace policies.

\section{Development of the WA Construct}

The WA construct was formally developed in the 1980s by Finnish researchers studying aging, work, and retirement (Ilmarinen et al., 1991b). In their study, they found that WA declined over an 11-year period, and that these declines were particularly prevalent among older employees and those working in blue-collar jobs (Ilmarinen, Tuomi, \& Klockars, 1997). Interestingly, similar to research on other age-related differences (e.g., cognitive changes), WA trajectories varied substantially across individuals over the study period (Ilmarinen et al., 1997). These findings suggested that WA trajectories may be influenced by a number of contextual, workplace, and individual difference factors.

Although early research focused on how WA relates to health, retirement, and disability, Feldt and colleagues (2009) found that psychosocial workplace factors such as organizational climate and job control predicted WA trajectories over a 10-year period. Drawing on these findings, researchers began calling for additional work to investigate the impact of psychosocial workplace factors on WA (Feldt, et al., 2009; Ilmarinen, 2009). Since then, researchers have begun to answer these calls (e.g., Airila et al., 2014; 
McGonagle et al., 2015). However, in doing so, the manner in which WA is measured has shifted in fundamental ways.

\section{Measurement of Work Ability}

As noted, the growth in WA research has coincided with a proliferation of WA measures that contain substantially different components of WA. Specifically, the following measures have been used to assess WA: the Work Ability Index (Ilmarinen et al., 1991; Morschhäuser \& Sochert, 2006); permutations of it that assess perceived WA, such as the Work Ability Score (Ahlstrom, Grimby-Ekman, Hagberg, \& Dellve, 2010) and "perceived WA" (McGonagle et al., 2015); as well as holistic assessments of WA, such as the Work Ability-Personal Radar (WA-PR; Ilmarinen, Ilmarinen, Huuhtanen, Louhevaara, \& Näsman, 2015) and Work Ability Survey-Revised (WAS-R; Noone, Mackey, \& Bohle, 2014).

Work Ability Index (WAI)

The most commonly used measure of WA is the Work Ability Index (WAI), which has been translated into over 26 languages and is used worldwide (Ilmarinen et al., 1991a; Morschhäuser \& Sochert, 2006). The WAI was formed with the goal of assessing "How good are workers at present and in the near future and how able are they to do their job with respect to work demands, health, and mental resources?” (Ilmarinen et al., 1997). As such, it incorporates several factors aimed at assessing both objective and subjective components of WA. Despite its widespread use, the WAI is problematic for at least four reasons. First, it combines objective health indicators (e.g., actual diagnosed diseases) and perceptual assessments of WA (e.g., self-ratings) into an overall WA score, 
despite evidence suggesting that a two-factor structure is more psychometrically sound (Martus, Jakob, Rose, Seibt, \& Freude, 2010). Second, the WAI is long, containing 59 items including the health conditions checklist and taking 10-15 minutes to complete (Morschhäuser \& Sochert, 2006). Third, the WAI includes sensitive health information (e.g., the health condition checklist) that respondents may feel uncomfortable sharing, particularly with their employer. Fourth, WA dimensions are scored using different response formats (e.g., 0-10 and 1-5) and weighting systems (e.g., multiplying dimension scores based on occupation), but the scores are then summed and converted into ordinal categories (i.e. quartiles), ranging from 7 to 49 ("poor" $=7$ - 27, "moderate" $=28$ - 36, "good" $=37-43$, and "excellent" $=44-49$ ). In terms of psychometric properties, this is problematic because it leads to a loss of information and decreased measurement variability.

Perceived WA Measures

In order to address the shortcomings of the WAI, researchers have adapted it in a number of ways, typically focusing on measures of perceived WA. One common adaptation is to use a single item from the WAI: "Describe your current work ability compared to your lifetime best," which is termed the Work Ability Score (Ahlstrom et al., 2010). However, using this single item completely removes the objective health component of the WAI, and thus may not be sufficient for addressing WA. Further, using a single item likely increases measurement error due to the bias inherent in respondents' self-assessments (Donaldson \& Grant-Vallone, 2002). 
Within the organizational psychology literature, researchers have used a variety of perceived WA measures, which are permutations of the WAI. For example, Weigl and colleagues (2013) assessed WA using the following two items from the WAI: "WA in relation to the physical demands of the job" and "WA in relation to the mental demands of the job" (Weigl, Müller, Hornung, Zacher, \& Angerer, 2013). As another example, Palermo and colleagues (2013) assessed WA using a four-item measure that included two perceived WA items from the WAI plus two items in which respondents rated their expected WA five and 10 years in the future (Palermo et al., 2013). Finally, McGonagle and colleagues $(2014,2015)$ used a four-item perceived WA measure that includes the first three items from the WAI and a new item: "Current WA in relation to the social skill demands of one's job" (McGonagle et al., 2014, 2015). These studies have begun to form the foundation of WA research within the organizational sciences.

Holistic Measures of Work Ability

Additional WA measures, such as the WA-Personal Radar (WA-PR; Ilmarinen, et al., 2015) and WA Survey-Revised (WAS-R; Noone et al., 2014), are based on a more comprehensive approach to assessing WA, where contextual factors (e.g., social support, flexibility of working time) are included in the measure of WA. These measures, however, operationalize WA so broadly that they subsume theoretical antecedents of WA (e.g., work characteristics) within the WA measure. Thus, we argue that the overly broad content included in these measures leads to construct contamination. Summary 
WA has been operationalized in a number of ways, varying in terms of length, the inclusion of objective versus perceived components, and the breadth of content in the measure. Despite these differences, research has shown that WA is related to a host of important individual (e.g., health, quality of life; Ahlstrom et al., 2010) and organizational (e.g., job resources, job attitudes, retirement; McGonagle et al., 2015; Sell, 2009; Tuomi, Huuhtanen, Nykyri, \& Ilmarinen, 2001) factors. However, while there are qualitative reviews of the WA concept (e.g., Cadiz et al., 2019), there has not been a systematic empirical summary of this literature, allowing for the investigation of the nomological network of WA or how the nomological network of WA is affected when using the WAI versus measures of perceived WA. This is a substantial problem given the large and accumulating body of research using both the WAI and perceived WA. What Is Work Ability?

Taken together, our overview of WA measures poses the basic question. What is work ability? Clearly, researchers have measured WA in several ways, and this stems in part from the wide range of disparate disciplines in which the WA concept has evolved. Drawing on these different definitions, we define WA simply as an individuals' ability to meet the requirements of their job. That is, can a person fulfill the given requirements of their job? To best assess WA, one might consider both subjective and objective indicators of WA as is done with the WAI. However, assessing health conditions as an objective indicator of ability to work is questionable, as those health conditions are assessed on their own and not within the context of the specific job requirements. For example, a leg injury would have a vastly different effect on a warehouse worker compared to a software 
engineer. Thus, we argue that perceptions of WA may provide a sufficient assessment of WA and that perceptions of WA are important in their own right; this is consistent with the vast majority of constructs are evaluated within the organizational sciences (e.g., perceptions of job resources, job demands). With WA more succinctly defined, we turn to the Job Demands-Resources Model as a framework for examining the correlates of WA. Towards a Conceptual Understanding of the Work Ability Construct: The Job DemandsResources Model

Although early WA research was primarily atheoretical, researchers have recently begun applying the job demands-resources (JD-R) model (Bakker \& Demerouti, 2007; Demerouti et al., 2001) as a framework to build a theoretical understanding of WA (e.g., McGonagle et al., 2015). According to the JD-R model, job demands are characteristics of the job (e.g., workload) that require sustained effort or skill and result in physiological or psychological costs. In contrast, job resources (e.g., autonomy) are aspects of the job that aid in accomplishing work goals, reducing job demands, or encouraging personal growth (Demerouti et al., 2001). Although job demands are not necessarily detrimental, they can lead to increased strain and decreased motivation. In contrast, job resources can increase motivation, facilitate personal growth, and buffer against the negative effects of job demands (Bakker \& Demerouti, 2007). Since its initial development, researchers have extended the JD-R model to include personal resources (e.g., health, optimism) that individuals can draw upon to meet the demands of their job (Demerouti, \& Bakker, 2011; Xanthopoulou, Bakker, Demerouti, \& Schaufeli, 2007). In addition, the JD-R model includes health impairment and motivational pathways that serve as mediators between 
resources and demands and job outcomes (e.g., Bakker \& Demerouti, 2007; Demerouti \& Bakker, 2011).

Applying the JD-R Model to Work Ability

Because WA entails a person's ability to meet the demands of their job (Ilmarinen et al., 1991; Ilmarinen, 2009), the JD-R model offers a useful framework for investigating the theoretical antecedents and outcomes of WA. We contend that WA can be incorporated into the JD-R model's health impairment and motivational pathways, where WA is influenced positively by job and personal resources and negatively by job demands (see Figure 1). As such, we view the antecedents of WA as existing in four broad categories within the JD-R framework: job demands, job resources, psychosocial personal resources, and health-based personal resources. We also examined age as a fifth antecedent. Only some of these antecedents have been examined in past research on WA (e.g., McGonagle et al., 2015), and a comprehensive quantitative review of their relation to different WA measures has not been conducted. In addition, our study includes additional theoretical outcomes, such as job attitudes, job performance, work motivation, and strain, which have not been the primary focus on WA research in the past. Although we acknowledge the directionality of the relations between WA and certain correlates may be reciprocal (e.g., engagement; Airila et al., 2014), we use the JD-R framework to organize our hypotheses, and as such, discuss our hypotheses in terms of theoretical antecedents and outcomes.

Theoretical Antecedents of Work Ability 
Job demands. We identified five job demand categories related to WA: quantitative, mental/emotional, physical, environmental, and workplace mistreatment. First, quantitative job demands such as time pressure and having a large quantity of tasks require high effort, drain the resources required to meet the demands of the job, and can lead to lower WA (McGonagle et al., 2015). Second, mental/emotional demands include ambiguity, direct conflict in one's defined role, and hiding one's emotions at work (Coverman, 1989; Eatough, Chang, Miloslavic, \& Johnson, 2011). Third, physical demands require physical effort (Bakker, Demerouti, Taris, Schaufeli, \& Schreurs, 2003), while the fourth category of environmental demands includes working in taxing environments, such as those that are hot or noisy (Tuomi et al., 2004). Fifth, workplace mistreatment refers to any form of aggressive behavior or mistreatment (e.g., incivility, confrontations, bullying) by customers, coworkers, or supervisors. In line with the JD-R, we argue that these demands will be negatively related to WA because individuals need to draw on their available resources to accomplish work tasks when faced with these demands.

\section{H1: Job demands will be negatively related to WA.}

Job resources. We identified seven categories of job resources that may relate to WA: coworker support, supervisor support, job control, task resources, rewards, perceptions of justice, and organizational climate. Coworker support and supervisor support refer to perceived or actual assistance from others (Karasek, Triantis, \& Chaudhry, 1982). Job control refers to decision-making freedom (Karasek, 1979) and may aid individuals in maintaining WA throughout the lifespan as functional capacities, 
interests, and knowledge change. Task resources, such as task variety and significance, can help build WA by facilitating skills acquisition and promoting a sense of accomplishment. Similarly, rewards such as promotion and adequate compensation as well as perceptions of justice should lead to increased WA. Finally, positive organizational climate may lead to greater WA by facilitating workers in meeting their work demands. In line with the JD-R, we predict that these job resources will be positively associated with WA.

H2: Job resources will be positively related to WA.

Psychosocial personal resources. Previous research has identified personal resources as perhaps the most strongly related resources to WA (McGonagle et al., 2015). Due to the extensive literature on health-based personal resources and WA, as well as the inclusion of health indicators in the WAI, we differentiate psychosocial personal resources and health-based personal resources in our study. Psychosocial personal resources are individual factors thought to aid workers in successfully fulfilling their work roles (Xanthopoulo et al., 2007). We identified ten of these resources: core selfevaluations, job self-efficacy, conscientiousness, positive traits (e.g., optimism), cognitive functioning, social support (non-work), coping, negative traits (e.g., hostility), grit/resilience, and selection, optimization, and compensation behaviors. This set of psychosocial personal resources expands upon previous work that has investigated a range of WA antecedents to include non-work social support, SOC behaviors, and cognitive functioning. As an example, core self-evaluations, which are an individual's consistent appraisal of themselves and their abilities across situations (Judge \& Bono, 
2001), have been linked to greater WA (Palermo et al., 2013). In addition, although limited research has been conducted on WA and personality, some research has uncovered positive links between WA and personality indicators such as conscientiousness (e.g., McGonagle et al., 2015), and other positive traits (e.g., optimism; Mache, Danzer, Klapp, \& Groneberg, 2013). Psychosocial personal resources can also take the form of behaviors aimed at adapting to one's changing abilities, such as selection, optimization, and compensation (SOC) behaviors (Baltes, 1997), as well as ability-based traits (e.g., cognitive functioning; Nygård, Eskelinen, Suvanto, Tuomi, \& Ilmarinen, 1991). These resources can be drawn upon to meet the demands of the job, and thus, we argue that they will be positively related to WA.

H3: Psychosocial personal resources will be positively related to WA.

Health-based personal resources. Due to WA's roots in occupational medicine, health-based personal resources have been studied a great deal in relation to WA, and some health indicators are included in the WAI and have been commonly studied as antecedents to perceived WA (Abdolalizadeh et al., 2012; Bethge, Radoschewski, \& Gutenbrunner, 2012a; Morschhäuser \& Sochert, 2006; von Bonsdorff, Huuhtanen, Tuomi, \& Seitsamo, 2009). In line with this research, an individual's health is perhaps their most important personal resource, as it influences everyday functioning and their ability to perform job tasks. Indeed, indicators of general, physical, and mental health (e.g., self-ratings, BMI, pain symptoms) have been linked to WA (Airila, Hakanen, Punakallio, Lusa, \& Luukkonen, 2012; Bethge et al., 2012a; El Fassi et al., 2013), such that indicators of better health are positively related to WA. In addition, health behaviors 
that positively impact one's personal resources, such as proper diet, physical activity, and refraining from smoking, are also likely related to WA. For example, refraining from smoking and excessive alcohol use and increased exercise are related to better health (Rimm, Klatsky, Grobbee, \& Stampfer, 1996; Schane, Ling, \& Glantz, 2010). Thus, we argue that these health-promoting behaviors should directly improve WA by increasing an individual's resource pool which can be draw upon to meet the demands of their job.

H4: Health-based personal resources, including specific indicators of good health and positive health behaviors, will be positively related to WA.

Age. Because the aging workforce was the initial impetus for studying WA, and early research identified it as a key risk factor for poor WA (Ilmarinen et al., 1991a), we anticipate that chronological age will be negatively related to WA. Chronological age is generally associated with declines in physical abilities (Millanvoye, 1998) and certain cognitive abilities (e.g., fluid intelligence; Schaie \& Willis, 1993). Further, age is also associated with greater risk for chronic health conditions (e.g., chronic back pain;

Manchikanti, Singh, Datta, Cohen, \& Hirsch, 2008), which are associated with lower levels of WA (El Fassi et al., 2013). Thus, we view age as a proxy for other developmental and experiential constructs (e.g., physical/cognitive abilities) and hypothesize that it will be negatively related to WA.

H5: Age will be negatively related to WA.

Theoretical Outcomes of Work Ability

According to the JD-R, job and personal resources lead to positive job outcomes via motivation, whereas job demands lead to negative outcomes via strain (e.g., burnout). 
We argue that WA influences work outcomes in a similar manner and serves as a link between job resources and job demands and job outcomes. Thus, in line with the JD-R, WA should relate to job outcomes including job attitudes, performance, strain, motivation, and withdrawal behaviors. First, we identified three job attitudes - job satisfaction (McGonagle et al., 2015), as well as organizational commitment, and the negative attitude of over-commitment - as being related to WA (e.g., Palermo et al., 2013). Second, although few studies have examined the WA-job performance relationship, we argue that if an individual has low WA, it is likely that their actual job performance will suffer as well. Third, according to the JD-R, strain (e.g., burnout, fatigue) results from an imbalance of demands and resources, where an individual experiences high demands and insufficient resources to meet those demands (Bakker \& Demerouti, 2007). Thus, we expect lower levels of WA to relate to higher levels of strain including fatigue, perceived stress, and burnout. Fourth, the JD-R model posits that motivation is positively influenced by job resources (Demerouti et al., 2001), and we argue that WA operates in a similar manner, enabling individuals to allocate more resources to their work and that those with higher WA will report higher motivation (e.g., Airila et al., 2014; Hakanen et al., 2006; Emmerich, \& Rigotti, 2017). Finally, because exit from the workforce (e.g., disability, retirement) was one of the key outcomes originally investigated with WA, and WA has been shown to negatively relate to these exit attitudes and behaviors (e.g., Ilmarinen et al., 1991b, 1997), we examined the relation between WA and exit intentions and behaviors. 
H6: WA will be positively related to attitudinal, motivational, and behavioral job outcomes, and negatively related to strain and exit intentions and behaviors.

Moderators

As noted above, WA has been studied in variety of settings and measured in a number of different ways. Next, we investigate the extent to which relationships between WA and related constructs are dependent on two factors: occupation type and type of WA measure used.

Occupation type. Occupation type has been identified as a risk factor for low WA, such that those working in blue collar jobs (primarily physically demanding) have lower levels of WA compared to those in white collar jobs (primarily mentally demands; Ilmarinen et al., 1997). Conceptually, the type of occupation an individual works in may also serve as a moderator of the relationships between WA and its correlates. Given our conceptualization of WA as an individual's ability to meet the demands of their job, the types of demands associated with a given job should play a critical role in an individual's WA. For example, although we anticipate that physical and mental health will be negatively related to WA, physical health may be more important in blue-collar jobs, and as such, the expected negative relationship between physical health and WA may be stronger. In contrast, for white-collar jobs, the relation between mental health and WA may be stronger. Although we expect occupation type to moderate the relationships between WA and its correlates, we believe the specific directions will vary depending on the specific correlate, and thus we pose this as a research question. 
Research Question: Does occupation type moderate the relationships between WA and its antecedents and outcomes?

Measure type. At a conceptual level, we have discussed the need to distinguish between objective and perceived WA. Thus, we also empirically compare the nomological network of WA based on the most common types of WA measures: the WAI and perceived measures of WA. A small number of studies have descriptively compared how strongly the WAI is related to specific correlates relative to perceived WA, but these studies have been limited to specific samples and a limited number of correlates, and have not empirically tested for the incremental validity of the WAI and perceived WA (e.g., Ahlstom et al., 2010; El Fassi et al., 2013). To develop hypotheses regarding the constructs for which the WAI or perceived WA would be more strongly related, we turn to the underlying theory behind them and the content of the measures.

Measures of perceived WA generally focus on one's ability to meet the demands of their job relative to their lifetime best, their current ability to meet specific types of job demands, and may also include perceptions about one's ability to meet those same demands at some point in the future (e.g., 2 years from now). In contrast, the WAI includes assessments of perceived WA, but was developed to incorporate a wide range of factors thought to underlie WA as well. As such, the WAI includes questions about current health conditions, absences from work, as well as "mental resources" such as depressive symptoms. Thus, when comparing the nomological network of the WAI compared to perceived WA, there is likely to be substantial overlap between the two constructs but also notable differences. Specifically, when investigating health-related 
correlates (e.g., subjective health, physical activity, disability status in the future), we anticipate that the WAI will demonstrate stronger associations with those health-related correlates than perceived WA. This is also due in part to the aforementioned construct contamination associated with the WAI. In contrast, perceived WA will be more strongly associated with job-focused correlates.

Hypothesis 7: The WAI will be more strongly related to health-based correlates than measures of perceived WA.

Hypothesis 8: Measures of perceived WA will be more strongly related to jobfocused correlates than the WAI.

Distinguishing Work Ability from Related Constructs

Because the WA concept has only recently been integrated into the organizational psychology literature, it is also important to distinguish it from existing, related constructs, and determine if a measure of perceived WA adds any unique value when considering other established variables. Although WA may overlap conceptually with some established constructs, such as perceived fit, general self-efficacy, and job selfefficacy, McGonagle and colleagues (2015) provided initial support for WA as a unique construct, finding a moderate correlation between perceived WA and general selfefficacy $(r=.40)$ as well as job self-efficacy $(r=.42)$. Thus, to provide additional evidence of WA as a unique construct and demonstrate the relative value of perceived WA, we conducted a second study to evaluate the utility of perceived WA in explaining unique variance in important health and job-related correlates. 
Perceived work ability compared to perceived fit. As discussed by Kristoff-Brown and colleagues (2005), the concept of perceived fit assumes that the match or fit between one's ability and their job leads to positive outcomes (e.g., job satisfaction, commitment). Perceived fit typically entails a matching or ideal "fit" between the person and the environment, including one's desires, needs, preferences, knowledge, skills, and the job (Edwards, 1991). Although the match between ability and work environment is important and likely related to WA, there are several key distinctions. For example, an aspiring musician working as a cashier may rate their perceived fit with being a cashier as relatively poor; that is, their skills and interests do not fit well with the job they are doing. Indeed, the musician may despise their job and dream of the day they are able to quit, but continue working there out of necessity. However, in the same scenario, the musician may have quite high perceptions of their WA - that is, their ability to meet the demands of their job. Thus, even though the fit between their job and their skills is poor, they are capable of meeting the demands of their job.

Perceived work ability compared to general self-efficacy. Following a review of the self-efficacy literature, Gist and Mitchell (1992) described self-efficacy as "beliefs in one's capabilities to mobilize the motivation, cognitive resources, and courses of action to meet the given situational demands (Wood \& Bandura, 1989)." Although perceived WA and general self-efficacy share a focus on perceptions of ability, self-efficacy is distinctly motivational, with an additional focus on taking action and allocating resources to accomplish goals. In contrast, WA is focused on meeting the basic demands of the job. Importantly, generalized self-efficacy is also considered to be consistent across contexts 
and can be considered an individual difference that people carry with them, similar to a personality trait. Within the JD-R, self-efficacy has been considered a personal resource that individuals can draw upon in order to meet job demands (Bakker \& Demerouti, 2017; Xanthopoulou, Bakker, \& Fischbach, 2013). These distinctions become clear when comparing items used to assess each construct. For example, consider this item from the Chen and colleagues (2001) general self-efficacy scale: "I will be able to achieve most of the goals that I have set for myself." Now consider this item that is commonly used to assess perceived WA: "How do you rate your current work ability with respect to the mental demands of your work?" (Ilmarinen et al., 1997, McGonagle et al., 2015, Morschhäuser \& Sochert, 2006). Clearly, both constructs and their respective measures assess self-perceptions, but they also differ substantially in the aforementioned ways. Although we anticipate generalized self-efficacy to be related to perceived WA, it is perhaps better considered an individual disposition, whereas perceived WA is far more context dependent.

Perceived work ability compared to job self-efficacy. To delineate between perceived WA and job self-efficacy, we point again to conceptual definitions as well as the measures for each construct. Measures of perceived WA generally focus on meeting basic demands. Further, measures of perceived WA often include an internal comparison to one's lifetime best as well as an estimate of anticipated WA in the future. On the other hand, job self-efficacy measures typically focus on meeting goals and overcoming challenges. Thus, job self-efficacy may be best at teasing apart motivational differences separating top performers from average-level employees. However, we argue that both 
pieces of information can be quite useful for investigating important individual- and work-related research questions.

Summary. In the present meta-analytic investigation, we also examine the empirical overlap among perceived WA, perceived fit, general self-efficacy, and job selfefficacy. Based on our conceptual review of the literature, we argue that due to its roots in occupational medicine, components of internal comparison, and focus on meeting basic requirements of a job, measures of perceived WA will explain unique variance in health-related correlates (self-rated health, burnout, intentions to apply for disability) as well as performance-based correlates (engagement, task performance, organizational citizenship behaviors), even when accounting for perceived fit, general self-efficacy, and job self-efficacy. However, we do not expect perceived WA to explain unique variance in job-related correlates that have a substantially affective component, including job satisfaction, affective commitment, and turnover intentions.

Hypothesis 9: When considering the related constructs, a) perceived fit, b) general self-efficacy, and c) job self-efficacy, perceived WA will explain incremental variance in health, engagement, burnout, intentions to apply for disability, and performance.

Hypothesis 10: When considering the related constructs a) perceived fit, $b$ ) general self-efficacy, and c) job self-efficacy, perceived WA will not explain incremental variance in job satisfaction, affective commitment, or turnover intentions.

Present Study 
In the present study, we first conducted in-depth meta-analyses to evaluate Hypotheses 1-8 and our Research Question regarding differences in the correlates of WA based on occupation type. This allowed us to establish the multidisciplinary nomological network of WA, compare the nomological network of WA when using the WAI and measures of perceived WA, and evaluate occupation type as a moderator. To provide additional evidence of the value of perceived WA, we conducted a second study that assesses Hypotheses 9-10 and evaluates the unique variance explained by perceived WA, when simultaneously considering the related constructs, perceived fit, general selfefficacy, and job self-efficacy.

Literature Search

\section{Study 1A Methods}

Studies assessing WA and its correlates were collected via PsycINFO and PubMed. We searched PsycINFO because our primary focus was the use of the WA construct within the organizational psychology literature, but due to its roots in occupational medicine, we also searched the PubMed database. We used the search terms work ability, workability, and work-ability. The search results were limited to studies published after 1980 and prior to September, 2017, studies published in English, and included journal articles, dissertations, thesis projects, and book chapters. Following this initial search, we reviewed reference lists from studies included in our search and reviewed the Society for Industrial and Occupational Psychology and European Association of Work and Organizational Psychology conference programs from 20122017. Finally, we submitted a call for unpublished studies including WA via the Occupational Health Psychology and Organizational Behavior listservs. After cross- 
checking for duplicate articles, these searches yielded 1,715 unique studies, 786 from PsycINFO, 922 from Pubmed, and 7 from other sources.

Screening and Coding Procedure

Screening and coding were conducted in three phases. During the screening process, each paper was screened for inclusion of a valid measure of WA and an effect size that could be converted into a correlation coefficient. Valid measures of WA were considered those that measured WA in line with the following conceptual definition of WA: One's physical and mental ability to perform job functions relative to the demands of their job (Ilmarinen et al., 1991a; Ilmarinen, 2009). Each article was also required to have enough information available to compute a correlation coefficient between WA and at least one other variable. During the screening phase, each article was reviewed by two members of the research team. Importantly, studies using only physician-diagnosed disability as a measure of WA rather than the WAI or perceived WA measures were not included in this study. Medical diagnoses are ratings of WA by a medical professional, not the individual, and generally are not considered within the scope of WA within the psychological literature.

Following the article screening, we identified $k=247$ studies which included $n=$ 312,987 individuals to analyze in our final dataset. During the first step of the coding process, each article was individually coded by two members of the research team, and disagreements between coders were resolved by a group discussion while reviewing the article in question. This coding was done at the highest level of specificity possible. For example, in the initial coding, the related constructs of self-esteem, self-efficacy, and 
other core self-evaluations (Judge \& Bono, 2001) were coded into their narrowest construct category (e.g., self-esteem, self-efficacy). After the first phase of coding was complete, the second phase of the coding involved grouping constructs into broader categories (e.g., core self-evaluations) for the purpose of analysis based on the number of available studies and the conceptual similarity among the constructs. This final step of creating construct groupings was done with at least two members of the research team looking at each variable included to determine the appropriateness for combining them into an overarching category. Moderator Variables

Regarding the moderators, the following coding decisions were made. As a moderator, WA measure type was separated into studies that used the full WAI versus those that measured perceived WA exclusively. This distinction was used because the literature has largely utilized either the WAI or a measure of perceived WA. For the occupation type moderator variable, we were unable to use the $\mathrm{O} * \mathrm{Net}$ classification of jobs since many samples included multiple specific jobs (e.g., bank employees, office workers). Rather, we coded occupation type as white-collar, which were considered to be primarily mentally demanding occupations (e.g., office, administrative), blue-collar, which were considered to be primarily physically demanding occupations (e.g., manual labor, front-line manufacturing), nurses/healthcare (e.g., nurses, nursing assistants, doctors), which were separated out due to the unique stressors associated with working in healthcare (i.e., mentally, physically, and emotionally challenging work), and studies that included mixed occupations (e.g., nationally representative samples) or other unique 
samples of employees (e.g., police personnel). The rationale for the distinction between these occupational groups is twofold. First, although there are differences within each occupation type, theoretically, the driving factor differentiating blue collar occupations from other job types is the physical nature of these roles. Indeed, these occupations generally require different training (e.g., trade schools vs. traditional college education) and often involve comparable demands (e.g., standing, lifting, and working with hands or heavy machinery) even if the specific skillsets differ (e.g., factory line vs. construction worker). A similar argument can be made for white collar occupations being primarily mentally demanding, and healthcare being a notoriously high-demand industry, mentally, physically, and emotionally. Second, based in part on the distinction between three occupation types in the WAI - namely, (1) primarily mentally demanding, (2) primarily physically demanding, or (3) both mentally and physically demanding - researchers sometimes present results for each of these occupational groups (Ilmarinen et al., 1997) or describe their study population as consisting of jobs that fit within the white collar (e.g., administrative assistants, officers, executives; Addley et al., 2014; technical administrative workers in education; Godinho et al., 2016) or blue collar dichotomy (e.g., manufacturing employees; Aranđelović, Nikolić, \& Stamenković, 2010). Meta-Analytic Approach

The meta-analyses were conducted using the Hedges and Olkin (1985) approach using Comprehensive Meta-Analysis 3.0 (CMA; Borenstein, Hedges, Higgins, \& Rothstein, 2014), wherein we converted all effect sizes into correlation coefficients. We selected the Hedges and Olkin approach for calculating weighted average correlations - 
as opposed to a psychometric approach (see Schmidt \& Hunter, 2014) - because of limitations in the information provided by researchers publishing articles on WA and the ways in which correlates of WA were conceptualized or operationalized. Researchers using the WAI often categorize outcome scores based on the four WAI categories ("poor" $=2-27$, "moderate" $=28-36$, "good" $=37-43$, and "excellent" $=44-49)$ and do not report estimates of reliability. Additionally, many of the antecedents and outcomes are observed variables with single-item indicators (e.g., age, BMI, smoking status, retirement and disability status). Thus, correction for attenuation due to unreliability in the predictor or outcome variable was either (a) not conceptually relevant or (b) not possible due to a lack of information provided in the empirical articles. The Hedges and Olkin approach thus provides a relatively conservative estimate of the effect sizes.

After converting all of the effect sizes to correlation coefficients, the metaanalyses were conducted in line with the proposed hypotheses. Consistent with prevailing meta-analytic practices, all analyses were conducted using random-effects models (Kepes, McDaniel, Brannick, \& Banks, 2013). Consistent with recommendations by Borenstein et al. (2009), when studies included multiple measures of a broader construct, we used the arithmetic mean to generate a single effect size estimate based on each measure's correlations (Borenstein, Hedges, Higgins, \& Rothstein, 2009). Similarly, when a study assessed a given relation at multiple time points (e.g., WA and BMI at baseline and one year), the sample-weighted average correlation was computed based on the relationships across time and served as the effect size estimate. 
In 13 of the included studies, we were able to code the relationships between WA and its correlates using both the WAI and a measure of perceived WA based on the same sample of participants. In these cases, the relationships between WA and its correlates were entered as independent effect sizes for each WA measure, despite the dependence between the estimates due to being based on the same sample. Although not ideal, Scammacca et al. (2014) showed that there were minimal differences in the overall estimated effect sizes when these steps were taken. Further, given that assessing the utility of these two approaches to measuring WA is a focal research question, we treated studies that reported effect size estimates for both the WAI and perceived WA as independent, even when they were derived from the same sample. The potential for publication bias was assessed via funnel plot diagrams and Begg and Mazumdar's (1994) rank correlation test for publication bias. These tests suggested only one instance of publication bias, which was for the relation between WA and future absenteeism; thus, some caution is warranted when interpreting that relation. However, we also note that this assessment included 58 tests for publication bias, only one of which suggested bias, and thus these estimates are likely largely free of publication bias. Moderator Analyses

To identify the existence of potential moderators for the hypothesized relations, the $Q$ statistic (Rosenthal, 1991) and $I^{2}$ index (Higgins \& Thompson, 2002; HuedoMedina, Sánchez-Meca, Marín-Martínez, \& Botella, 2006) were used as indicators of effect size heterogeneity that might be explained by moderators. The $Q$ statistic indicates the variability in in the underlying "true" effect sizes, with larger values indicating more 
heterogeneity (Huedo-Medina et al., 2006; Kepes et al., 2013) and significant values signaling heterogeneity in effect sizes beyond sampling error and the presence of moderators. The $I^{2}$ index indicates the magnitude of the variability among effect sizes, and can be interpreted as the percentage of observed variability that can be attributed to "true" variability, with values closer to $100 \%$ being consistent with the presence of moderators (Huedo-Medina et al., 2006; Kepes et al., 2013). Although these tests serve as indicators of the potential presence of moderators, the moderator analyses were conducted in each case where there was a sufficient number of studies. This is supported by findings from Hedges and Pigott (2001), suggesting the power to detect heterogeneity among effect size estimates can be low in meta-analyses, particularly when $k$ is relatively small.

The $Q$ statistic was also used to assess whether effect sizes showed significant variability across levels of categorical moderator variables. For the WA measure type moderator, studies were coded as using either the WAI or a perceived WA measure. Accordingly, a significant $Q$ statistic signals statistical differences in effect sizes between studies using the WAI measure versus a perceived WA measure. Of note, the occupation type moderator was coded into four categories: white-collar, blue-collar, nursing/healthcare, and mixed or other occupations. Given that the purpose of assessing occupation as a moderator is to determine for which occupation types a given factor is more strongly related to WA, we excluded the mixed occupation group from these analyses. Occupation type as a moderator was evaluated using the $Q$ statistic via pairwise tests between the white-collar, blue-collar, and nursing/healthcare occupation types. 
Study 1A Results

Meta-analyses were used to assess Hypotheses 1-8 and our Research Question. Results of the overall estimates are presented for the correlates of WA in terms of the theoretical antecedents and outcomes in Tables 1 and 2, respectively, as well as in Figure 2. Moderator analyses, separated by the theoretical antecedents and outcomes, are presented for the type of WA (see Tables 3 and 4) and occupation type (see Tables 5 and 6). We discuss the results of the moderator analyses, evaluating Hypotheses 7 and 8 and our Research Question, within the results for each main effect. We then provide a brief summary for each at the end of the results section. For parsimony, we note here that in each of the overall estimates aside from two associations - objective health indicators and alcohol consumption - the $95 \%$ confidence interval did not include zero. In addition, the $Q$ statistic and $I^{2}$ index indicated the presence of potential moderators for most associations, with the exceptions of those with cognitive ability, coping, and grit/resilience. In the following sections, we highlight findings from each hypothesis and research question.

Theoretical Antecedents of WA

Job demands. In support of Hypothesis 1, associations between job demands and WA were consistently negative $(\overline{\boldsymbol{r}}=-.205, k=55)$. Between the five job demand categories (i.e., quantitative demands, mental/emotional demands, physical demands, environmental conditions, workplace mistreatment), however, estimates did vary to some extent, with the weakest association for environmental conditions (e.g., hot and humid workplaces $)(\overline{\boldsymbol{r}}=-.110, k=10)$ and the strongest the association for mental/emotional demands (e.g., role conflict, and hiding emotions) $(\overline{\boldsymbol{r}}=-.270, k=12)$. Although the $Q$ 
statistic and $I^{2}$ index indicated the presence of moderators, WA measure type and occupation type did not explain significant variation in the relations between job demands and WA.

Job resources. Providing strong support for Hypothesis 2, job resources were consistently and positively related to WA $(\overline{\boldsymbol{r}}=.212, k=62)$. Across the seven job resource categories (i.e., coworker support, supervisor support, job control, task resources, rewards, justice perceptions, positive organizational climates), associations ranged from $(\overline{\boldsymbol{r}}=.197, k=11)$ for organizational climate to $(\overline{\boldsymbol{r}}=.272, k=15)$ for justice perceptions. Moderator analyses showed that the relation between job resources and WA was stronger in studies using the WAI $(\overline{\boldsymbol{r}}=.245, k=26)$ than in those using perceived WA measures $(\overline{\boldsymbol{r}}=.188, k=39)(Q=3.90, p=.049)$. Additionally, with regard to occupation type, job resources were more strongly related to WA for those working in white-collar occupations $(\overline{\boldsymbol{r}}=.273, k=13)$ than blue-collar occupations $(\overline{\boldsymbol{r}}=.172, k=10)$ $(Q=7.72, p=.005)$ and to a marginal extent, nursing/healthcare occupations $(\overline{\boldsymbol{r}}=.210, k$ =13) $(Q=2.93, p=.087)$. Drilling down to specific job resources, this effect was only present for job control $(Q=13.24, p<.001)$, which was more strongly associated with WA in white-collar occupations $(\overline{\boldsymbol{r}}=.298, k=10)$ than in blue-collar occupations $(\overline{\boldsymbol{r}}=$ $.140, k=7)$. Similarly, the relation between job control and WA was stronger for those in white-collar occupations compared to those in nursing/healthcare occupations $(\overline{\boldsymbol{r}}=.200, k$ =6) $(Q=5.56, p=.018)$.

Psychosocial personal resources. In support of Hypothesis 3, psychosocial personal resources were positively related to WA $(\overline{\boldsymbol{r}}=.253, k=39)$. Across the ten 
psychosocial personal resources (i.e., core self-evaluations, job self-efficacy, conscientiousness, cognitive functioning, positive traits, grit/resilience, negative traits, social support (non-work), coping, and selection, optimization, and compensation [SOC] behaviors $)$, core-self evaluations $(\overline{\boldsymbol{r}}=.377, k=11)$ and grit/resilience $(\overline{\boldsymbol{r}}=.371, k=3)$ were most strongly related to WA, whereas coping $(\overline{\boldsymbol{r}}=.113, k=3)$ and SOC behaviors were least strongly associated with WA $(\overline{\boldsymbol{r}}=.151, k=7)$. The association between overall psychosocial personal resources and WA did not differ by WA measure type. However, negative traits were less strongly related to WA in studies using the WAI $(\overline{\boldsymbol{r}}=-.199, k=$ 3) compared to those using perceived WA measures $(\overline{\boldsymbol{r}}=-.346, k=4)(Q=4.19, p=$ .041). The relation between psychosocial personal resources and WA was not moderated by occupation.

Health-based personal resources. We identified three broad categories of healthbased personal resources in addition to health behaviors which all positively related to WA and thus, provide strong support for Hypothesis 4: general health $(\overline{\boldsymbol{r}}=.383, k=87)$, mental health $(\overline{\boldsymbol{r}}=.434, k=43)$, physical health $(\overline{\boldsymbol{r}}=.311, k=84)$, and health behaviors $(\overline{\boldsymbol{r}}=.072, k=41)$. Within these categories, we identified ten specific health indicators: non-pain symptoms (e.g., dizziness, difficulty concentrating, and stomach problems), current health status (e.g., being free from negative health conditions), previous absenteeism, sleep, depressive symptoms, anxiety, physical ability, BMI, objective indicators (e.g., cholesterol), and pain-related symptoms as well as the three specific health behaviors physical activity, smoking (tobacco), and alcohol consumption. Note, health indicators reflecting poor health, such as depressive symptoms, were recoded as 
positive when assessing the overall category of mental health and coded as negative when reporting on the specific indicator (e.g., anxiety). Among these, depressive symptoms was most strongly related to WA $(\overline{\boldsymbol{r}}=-.460, k=14)$, whereas the weakest relations were with BMI $(\overline{\boldsymbol{r}}=-.125, k=31)$, physical activity $(\overline{\boldsymbol{r}}=.113, k=29)$, and smoking $(\overline{\boldsymbol{r}}=-$ $.080, k=31$ ). Objective indicators of physical health (e.g., cholesterol, blood pressure) and alcohol consumption were not related to WA $(\overline{\boldsymbol{r}}=.139, k=5,95 \%$ CI $[-.035, .305]$ and $\overline{\boldsymbol{r}}=-.007,95 \%$ CI [-.052, .037], $k=20$, respectively).

The WA measure type moderated the association between general health and WA, such that the effect was stronger in studies where the WAI was used $(\overline{\boldsymbol{r}}=.433, k=39)$ than in those that used a perceived WA measure $(\overline{\boldsymbol{r}}=.347, k=52)(Q=4.77, p=.029)$. In addition, when using the WAI, the relation between current health status and WA was stronger $(\overline{\boldsymbol{r}}=.476, k=10)$ than when using a perceived WA measure $(\overline{\boldsymbol{r}}=.290, k=25)$ $(Q=11.14, p=.001)$. Given that diagnosed illnesses are subsumed in the WAI, this discrepancy is not surprising, but it is important to note that perceived WA measures still demonstrated a moderate negative association with health status.

Occupation type also moderated several relations. Mental health was more strongly related to WA for those working in white-collar occupations $(\overline{\boldsymbol{r}}=.529, k=9)$ than for those working in blue-collar $(\overline{\boldsymbol{r}}=.400, k=6)(Q=7.99, p=.005)$ and nursing/healthcare occupations $(\overline{\boldsymbol{r}}=.396, k=5)(Q=7.14, p=.008)$. Similarly, occupation type moderated the relation between physical health and WA, and interestingly, the relation between physical health and WA was stronger in primarily white-collar occupations $(\overline{\boldsymbol{r}}=.512, k=9)$ than in blue-collar occupations $(\overline{\boldsymbol{r}}=.262, k=$ 
20) $(Q=6.33, p=.012)$. Occupation type also moderated the relation between health behaviors and WA when comparing blue collar occupations $(\overline{\boldsymbol{r}}=.109, k=13)$ to nursing/healthcare occupations $(\overline{\boldsymbol{r}}=-.011, k=4)(Q=5.98, p=.014)$. Further, physical activity was more strongly related to WA among those in blue-collar occupations $(\overline{\boldsymbol{r}}=$ $.154, k=8)$ than those in nursing/healthcare jobs $(\overline{\boldsymbol{r}}=-.008, k=3)(Q=5.21, p=.022)$, whereas there was a marginally significant difference between those in white-collar occupations $(\overline{\boldsymbol{r}}=.160, k=3)$ and nursing/healthcare occupations $(Q=3.14, p=077)$. Finally, smoking was more strongly and negatively related to WA among workers in blue-collar occupations $(\overline{\boldsymbol{r}}=-.108, k=9)$ than those in nursing/healthcare occupations $(\overline{\boldsymbol{r}}=-.022, k=3)(Q=5.72, p=.017)$.

Age. Overall, age was negatively related to WA $(\overline{\boldsymbol{r}}=-.114, k=111)$. However, the relationship between age and WA was not moderated by the WA measure type or occupation type. Although the effect of age was small, its relationship with WA was robust and consistent across contexts, providing support for Hypothesis 5. Theoretical Outcomes of WA

In line with the JD-R model, we hypothesized that WA is associated with workrelated outcomes as well. For clarity, we present these findings according to the following categories: job attitudes, job performance, motivation, strain, and exit intentions and behaviors. As described in the following sections, we found general support for Hypothesis 6, as WA was associated with the aforementioned outcomes in the expected directions. 
Job attitudes. Overall, WA was moderately and positively related to job attitudes $(\overline{\boldsymbol{r}}=.262, k=19)$. Specifically, the job attitudes of job satisfaction $(\overline{\boldsymbol{r}}=.283, k=10)$, organizational commitment $(\overline{\boldsymbol{r}}=.256, k=6)$, and over-commitment $(\overline{\boldsymbol{r}}=-.218, k=4)$ showed similarly-sized relations with WA. Note that over-commitment is a negative attitude representing unhealthy commitment to one's work and was thus reverse coded when computing the overall effect of WA on job attitudes. The association between job attitudes and WA was not moderated by WA measure type or occupation type.

Job performance. WA was positively related to job performance $(\overline{\boldsymbol{r}}=.324, k=9)$. Although this positive relation between WA and performance is potentially meaningful, it is important to note that these estimates consisted of self-report ratings of job performance. Although the $Q$ statistic and $I^{2}$ index suggest the potential for moderators, there was an insufficient number of samples to assess WA measure type and occupation type as moderators.

Motivation. WA was positively related to motivation, $(\overline{\boldsymbol{r}}=.304, k=16)$. This relation was not moderated by WA measure type or occupation type.

Strain. Strain was assessed as measures of fatigue, burnout, and perceived stress, and was consistently and negatively related to WA $(\overline{\boldsymbol{r}}=-.396, k=39)$. Specifically, fatigue $(\overline{\boldsymbol{r}}=-.416, k=17)$ and burnout $(\overline{\boldsymbol{r}}=-.444, k=14)$ were somewhat more strongly related to WA than perceived stress $(\overline{\boldsymbol{r}}=-.347, k=13)$. WA measure type did not moderate the relation between WA and strain, but occupation type did, such that strain was more strongly related to WA for white-collar $(\overline{\boldsymbol{r}}=-.481, k=8)$ versus blue-collar occupations $(\overline{\boldsymbol{r}}=-.309, k=10)(Q=4.54, p=.03)$. 
Exit intentions and behaviors. Exit intentions were negatively related to WA $(\overline{\boldsymbol{r}}=$ $-.263, k=21)$ and were operationalized as turnover intentions and retirement intentions. Retirement intentions $(\overline{\boldsymbol{r}}=-.241, k=9)$ and turnover intentions, $(\overline{\boldsymbol{r}}=-.273, k=13)$ shared similarly sized relations with WA $(\overline{\boldsymbol{r}}=-.241, k=9$ and $\overline{\boldsymbol{r}}=-.273, k=13$, respectively). Exit behaviors were operationalized as future absenteeism, disability status, and retirement. Importantly, in all studies, these effects were estimated longitudinally with work status measured at some point in the future after WA was measured. Overall, exit behaviors were negatively related to WA $(\overline{\boldsymbol{r}}=-.257, k=20)$. Specifically, WA was related to future disability status $(\overline{\boldsymbol{r}}=-.384, k=6)$, absenteeism $(\overline{\boldsymbol{r}}=-.225, k=13)$, and retirement $(\overline{\boldsymbol{r}}=-.189, k=4)$.

The relation between WA and exit intentions trended towards being moderated by WA measure type. Specifically, when using the WAI $(\overline{\boldsymbol{r}}=-.313, k=15)$, studies showed a marginally stronger relation between WA and exit intentions when compared to those using a perceived WA measure $(\overline{\boldsymbol{r}}=-.139, k=6)(Q=3.35, p=.067)$. The relation between WA and exit intentions was not moderated by occupation type. WA measure type did not moderate the relation between WA and exit behaviors. However, WA was more strongly related to future disability status in studies using the WAI $(\overline{\boldsymbol{r}}=-.534, k=$ 4) than in those using a perceived WA measure $(\overline{\boldsymbol{r}}=-.236, k=3)(Q=5.56, p=.018)$. Notably, although the relation between WA and future disability status was markedly smaller in studies using a measure of perceived WA, perceived WA still demonstrated a significant relationship with future disability status. 
Item-level WA analysis. To further expand upon our investigation of the impact of the WA measure on the nomological network, we also wanted to determine the extent to which individual perceived WA items correlated with the theoretical antecedents and outcomes identified in this study. Based on the available studies, we were able to perform these analyses for the perceived WA item "please rate your current work ability compared to your lifetime best." Overall, these results were remarkably strong for a single-item indicator. Specifically, job demands $(\overline{\boldsymbol{r}}=-.214, k=15)$, job resources $(\overline{\boldsymbol{r}}=$ $.172, k=17)$, and health based personal resources, general health $(\overline{\boldsymbol{r}}=.360, k=35)$, mental health $(\overline{\boldsymbol{r}}=.397, k=11)$, physical health $(\overline{\boldsymbol{r}}=.230, k=19)$, and health behaviors $(\overline{\boldsymbol{r}}=.103, k=13)$ all demonstrated consistent relations with the single perceived WA item. However, when considering psychosocial personal resources, using a longer perceived WA measure demonstrated a substantially stronger relation with WA $(\overline{\boldsymbol{r}}=.275$, $k=24)$ than the single perceived WA item $(\overline{\boldsymbol{r}}=.118, k=8)$. To facilitate greater understanding of individual perceived WA items, we expand upon these findings in Study 2.

Summary of Meta-Analytic Findings

In summary, with the theoretical antecedents of WA, we found that the relations between WA and job demands were generally negative, and the relations between WA and job and psychosocial personal resources were consistently positive. The relations between WA and health-based personal resources were mixed, and WA was negatively related to age. Regarding the theoretical outcomes of WA, we found that WA positively related to job attitudes, performance, and motivation, and negatively related to strain and 
exit behaviors. Several of these relationships were moderated by WA measure type and/or occupation type.

Measure type moderator. Hypothesis 7 posited that when assessed via the WAI, WA would be more strongly related to health-based correlates than when assessed via a perceived WA measure. This hypothesis was generally supported. Studies using the WAI showed stronger relations between WA and general health. Current health status was more strongly related to WA among studies using the WAI than those using a perceived WA measure. Last, studies using the WAI showed a stronger relation to future disability status than those using a perceived WA measure. Although these findings are not surprising given the health-indicators imbedded within the WAI, it is important to note that perceived WA measures were also significantly related to the vast majority of these health-focused correlates.

Hypothesis 8 posited that job-related correlates would be more closely related to WA when assessed via a perceived WA measure than the WAI. However, this hypothesis was not supported. In fact, when considering job resources, the relation between WA and job resources was stronger among studies utilizing the WAI compared to those using a perceived WA measure. Thus, perceived WA measures provided similar results compared to the WAI when investigating job-related correlates and thus did not provide a notable advantage over using the WAI.

Occupation type moderator. There was moderate support for occupation type moderating the relations between WA and its correlates. Specifically, job resources were more strongly related to WA for those working in white-collar as opposed to blue-collar 
occupations. Drilling down to specific psychosocial job resources, this effect was only present for job control, which was more strongly associated with WA in white-collar than in blue-collar occupations. To a marginal extent, the relation between job resources and WA was also stronger among white-collar than among nursing and healthcare occupations, but when considering job control specifically, job control was more strongly related to WA in white-collar occupations than in nursing and healthcare occupations. Interestingly, when investigating the health-based personal resources of mental health and physical health, both were more strongly related to WA for those working in white-collar occupations than those in blue-collar occupations. In addition, mental health was more strongly related to WA among those working in white-collar occupations than those in nursing/healthcare occupations. Occupation type also moderated the relation between health behaviors and WA. When comparing blue-collar to nursing and healthcare occupations, health behaviors overall, physical activity, and smoking were more strongly related to WA among those in blue-collar occupations compared to those in nursing and healthcare. When comparing white-collar occupations to nursing/healthcare, there was also a marginally significant difference in the relation between physical activity and WA, such that the relation was somewhat stronger among those in white-collar occupations. Occupation type also moderated the relation between WA and strain, such that strain was more strongly related to WA for white-collar versus blue-collar occupations. 
Study 1B

Given support from Study 1 for using measures of perceived WA, we wanted to compare perceived WA with similar yet theoretically distinguishable constructs, namely perceived fit, general self-efficacy, and job self-efficacy (Hypotheses 9 and 10). Although we found in our meta-analysis that WA was related to job self-efficacy $(\overline{\boldsymbol{r}}=.352, k=6, \mathrm{~N}$ $=1,666,95 \% \mathrm{CI}[.284, .417])$ and core self-evaluations $(\overline{\boldsymbol{r}}=.377, N=5,032,95 \% \mathrm{CI}$ $[.294, .455])$ we were limited in our ability to examine Hypotheses 9 and 10 due to relatively few studies with appropriate covariates also included in the study. Therefore, to evaluate Hypotheses 9 and 10, we conducted a follow-up study to assess the discriminant validity of perceived WA relative to the theoretically related yet distinguishable constructs of perceived fit, general self-efficacy, and job self-efficacy. In doing so, we evaluated whether perceived WA explained incremental variance beyond perceived fit, general self-efficacy, and job self-efficacy in the following correlates: health, engagement, exhaustion, intentions to apply for disability, performance, job satisfaction, affective commitment, and turnover intentions. Note that our focus in Study 2 was perceived WA rather than the WAI given their similar performance with the covariates in Study 1 and the greater likelihood of using perceived WA in organizational research.

Study 1B Methods

Participants and Procedures

Data were collected via Amazon's Mechanical Turk (MTurk) from 334 participants using the following requirements, 97\% HIT (Human Intelligence Task) approval, speaking English, having current, paid employment, and being located within 
the United States. To achieve a more age-representative sample, 50 of the participants were restricted to being at least 50 years old via the TurkPrime panel study option. After removing participants due to study restrictions and missed attention check items a total of 282 participants remained. Participants were on average $42.79(S D=12.88)$ years old, $84.4 \%$ white, and $51.4 \%$ male, and the majority worked in primarily psychologically (66.7\%) or physically and psychologically demanding jobs (25.5\%). All participants were employed and worked an average of $38.59(S D=10.47)$ hours per week.

Measures

Unless noted otherwise, items were assessed on a 7-point scale with scales coded to represent higher levels of the assessed construct. All measures demonstrated good internal consistency greater than $\alpha=.75$. See Table 7 for specific values. Perceived $W A$ was evaluated using the four item measure based on the original subjective assessment of WA from the WAI (Ilmarinen et al., 1991) and expanded upon by McGonagle and colleagues $(2014,2015)$. The first item asks participants to rate their "current work ability compared to your lifetime best." The other three items ask about WA relative to specific job requirement domains via the following questions "How do you rate your current work ability with respect to the 1) mental, 2) physical, 3) interpersonal, demands of your job?" These items are scored from $(0=$ completely unable to work $)$ to $(10=$ work ability at its best). Perceived fit was evaluated using a 9-item measure from Cable and DeRue (2002). A sample item is "My abilities and training are a good fit with the requirements of my job." General self-efficacy was evaluated using an 8-item scale developed by Chen and colleagues (2001). A sample item is "When facing difficult tasks, 
I am certain that I will accomplish them." Job self-efficacy was evaluated using the job self-efficacy scale from Rigotti, Schyns, and Mohr (2008). A sample item is "Whatever comes my way in my job, I can usually handle it."

General, mental, and physical health were evaluated with three, single-item indicators asking participants to rate "Overall, how would you rate your 1) general 2) mental, and 3) physical health?" Similarly, relative health was evaluated with the item "Compared to others your age, how would you rate your health?" Exhaustion was assessed via the Oldenburg Burnout Inventory (Demorouti et al., 2001). A sample item is "During my work, I often felt emotionally drained." Disability intentions were evaluated with three items adapted from the Hom and colleagues (1984) retirement intentions scale. Engagement was evaluated using the nine-item version of the Utrecht Work Engagement Scale (UWES; Schaufeli et al., 2006). A sample item is "At my work, I feel bursting with energy." Task performance was evaluated with three items from Griffin and colleagues (2007). This measure included items such as "In my job, I carry out the core parts of my job well." Organizational citizenship behaviors toward the organization (OCBO) and the individual (OCBI) were assessed with seven items each from the scale by Williams and Anderson (1991); sample items included "At work, I help others who have heavy workloads" (OCBI) and "Give advanced notice when unable to come to work" (OCBO). Job satisfaction was assessed with three items from Cammann and colleagues (1979) such as "In general, I like working here." Affective organizational commitment was assessed with eight items from Allen and Meyer (1990), "I really feel as if this organization's problems are my own". Turnover intentions were assessed with three 
items adapted from Hom and colleagues (1984), including "I often think about quitting this organization."

\section{Study 1B Results}

To evaluate Hypotheses 9-10, a series of sequential multiple regressions were conducted. Specifically, in steps 1-3 of each model, perceived fit, general self-efficacy, and job self-efficacy were added sequentially to the equation. In step 4, perceived WA was added to the equation to determine if perceived WA explained unique variance in one of the correlates (e.g., burnout, OCBO). Due to the potential for multicollinearity among the predictor variables, we also assessed the variance inflation factor (VIF). When including all four variables in step 4, the VIF for each variable remained comfortably under the conventional rules of thumb in which a VIF of 10 is considered severe (Marquardt, 1970) and a VIF of 5 is considered potentially concerning (Menard, 1995). General self-efficacy and job self-efficacy showed the highest collinearity with VIFs equal to 2.60 and 2.55 , respectively, whereas perceived fit and perceived WA showed less multicollinearity with VIFs equal to 1.243 and 1.451 , respectively. Further, removing either general self-efficacy or job self-efficacy from the regression model did not change the pattern of results regarding the unique variance explained by perceived WA. Below, we summarize the results of these analyses. See Table 7 for the correlations among study variables, including at the individual item level, and Table 8 for results of the regression analyses. As shown in Table 7, perceived WA showed strong correlations with general self-efficacy (.53) and job self-efficacy (.51), and a lower but significant correlation with person- job fit (.28). In addition, from an item perspective, Table 7 shows that the WA 
items focused on current WA relative to lifetime best, mental demands, and interpersonal demands were significantly related to all of the correlates. Interestingly, the WA item focused on physical demands had the smallest correlations, and showed a near zero correlation with organizational commitment and turnover intentions.

When considering the four related constructs together, perceived WA explained unique variance in the majority of correlates. Specifically, perceived WA explained unique variance in general health $\left(\beta=.401, \Delta R^{2}=.111, p<.001\right)$, mental health $(\beta=$ $\left..225, \Delta R^{2}=.035, p<.001\right)$, physical health $\left(\beta=.410, \Delta R^{2}=.116, p<.001\right)$, and relative health $\left(\beta=.324, \Delta R^{2}=.072, p<.001\right)$. Similarly, perceived WA explained unique variance in exhaustion $\left(\beta=-.259, \Delta R^{2}=.046, p<.001\right)$ and disability intentions $(\beta=$ $\left..384, \Delta R^{2}=.102, p<.001\right)$

Regarding the performance-related correlates, perceived WA again explained unique variance beyond perceived fit, general self-efficacy, and job self-efficacy in engagement $\left(\beta=.122, \Delta R^{2}=.010, p=.013\right)$, task performance $\left(\beta=.205, \Delta R^{2}=.029, p<\right.$ $.001)$, OCBOs $\left(\beta=.286, \Delta R^{2}=.056, p<.001\right)$, and OCBIs $\left(\beta=.162, \Delta R^{2}=.018, p=\right.$ $.006)$.

In contrast, when considering attitudinal variables, job satisfaction $\left(\beta=.048, R^{2}=\right.$ $.02, p=.284)$, affective commitment $\left(\beta=.031, R^{2}=.001, p=.524\right)$, and turnover intentions $\left(\beta=-.040, R^{2}=.001, p=.451\right)$ perceived WA did not explain unique variance beyond the other variables. Taken together, these results generally support Hypotheses 910 and demonstrate that even when considering related factors, perceived WA provides unique value to organizational scholars. 


\section{General Discussion}

With its origins in occupational medicine, the concept of WA has recently taken root in the organizational psychology literature. However, the WA concept suffers from multiple definitions and operationalizations across disciplines, and several measures have emerged, some with questionable psychometric and theoretical qualities. The present study addresses these issues by providing a quantitative summary of the WA literature and an examination of the measurement of WA, thereby advancing the literature on WA in several ways. First, it provides a comprehensive quantitative synthesis of the WA literature to date, reaching across the domains of organizational psychology and occupational medicine and including hundreds of studies, thousands of individuals, and multiple occupations. Second, this analysis allowed us to compare perceived WA measures and the WAI in terms of their relationship with dozens of theoretical antecedents and outcomes of WA, far beyond the most comprehensive past examinations of WA (e.g., McGonagle et al., 2015). In this way, this study clarifies the WA construct. Second, this study examines whether measures of perceived WA, which have been introduced recently as more psychometrically sound and relatively easy to administer, have a notably different nomological network than the long-established WAI. Third, this study investigates the moderating role of occupation type as a boundary condition to determine if the correlates of WA vary by occupational group. Fourth, we provide an indepth examination of perceived WA at the item level and show the unique variance explained by perceived WA compared to the conceptually related constructs of perceived 
fit, general self-efficacy, and job self-efficacy. We discuss each of these contributions below.

\section{Conceptual Clarification of Work Ability}

Unlike previous research, we argue that work ability as a construct is relatively straightforward, representing an individual's ability to meet the requirements of their job. As such, WA answers the fundamental question, "Can a person fulfill the basic and essential functions required for a given job?" However, this varies by the way WA is measured: The WAI focuses on a person's perception that they can fulfill their job requirements and also their current health problems, while perceived WA is focused on the person's perceptions only. As shown in our study, the factors that influence WA both the WAI and perceived WA - are wide-reaching and at times, complex. That is, WA is influenced by a host of personal factors (e.g., health, mental and physical ability, personality, and behaviors), organizational constructs (e.g., job resources, job control, support, perceptions of fairness, job demands, environmental conditions, and workplace mistreatment). However, those relations can vary based on how WA is measured and the occupation type of the sample.

Synthesis of the WA Literature and Theory of Work Ability

To date, there has been minimal integration of the medical and organizational psychology literatures on WA, and each has developed and investigated WA within its respective silo. We have bridged this gap by integrating the findings across these disciplines, reflecting the breadth of research investigating WA. 
Theoretical antecedents of WA. We found strong support for conceptualizing the nomological network of WA through the lens of the JD-R. Specifically, job demands were negatively related to WA, whereas job and personal resources were positively related to WA. Similar to McGonagle et al. (2015), we found stronger relations between WA and personal resources, with health-related personal resources having particularly strong relationships with WA. However, we also expanded upon McGonagle et al.'s (2015) original set of theoretical antecedents in many ways. Specifically, we found that job factors of an interpersonal nature, such as perceived justice and workplace mistreatment, as well as psychosocial personal resources, such as non-work social support, cognitive functioning, coping, and SOC behaviors are also important to consider when investigating WA. In addition, we highlighted specific health focused behaviors (e.g., physical activity, refraining from smoking) that are related to WA and thus might be used to promote it. Finally, we also investigated the utility of objective health indicators (e.g., glucose levels), as alcohol consumption, finding that they showed weak or non-significant relations with WA. Ultimately, this work greatly expands upon the theoretical antecedents identified as important for WA in previous research.

Theoretical outcomes of WA. The present study also examined the relationship between WA and a number of attitudinal (e.g., job satisfaction, commitment), motivational (e.g., engagement), strain-related, and behavioral (e.g., performance, exit behaviors, future disability status) outcomes. Specifically, we found that WA was a predictor of all of these outcomes, many of which - work engagement, strain, work motivation, and performance - had not been summarized in previous research on WA 
(e.g., McGonagle et al. 2015). Thus, this research situates WA as an important construct to consider not only when investigating employee exit behaviors, but also in contexts related to promoting motivation, reducing strain, and improving job attitudes and performance.

Comparison of the WAI versus Perceived WA

Comparing the WAI to measures of perceived WA and thus developing WA theory by empirically evaluating its nomological network was a critically important goal of our study. We found that the type of WA measure only moderated the relationship between WA and its correlates in five of the 58 assessed relationships; that is, the type of WA measure generally had little effect on the study results. That said, two health-focused variables, current health status and exit from the workforce via disability or pension status, were more strongly related to the WAI than to perceived WA, which is not surprising given that the WAI includes an in-depth assessment of health problems. Thus, although the relation between perceived WA and future disability status was still significant, perceived WA may be less useful for predicting disability pension claims, specifically. That said, most differences found between perceived WA and the WAI were relatively small, suggesting that perceived WA could serve as a useful and far simpler measure than the WAI. In addition, we argue that researchers would be better served assessing perceived WA in conjunction with measures of health (e.g., condition checklist, self-rated health), as opposed to using the WAI in which perceived WA and health are intertwined within the measure. This approach would likely retain the additional predictive power associated with the WAI, while allowing researchers to gain a more 
nuanced understanding of the antecedents of future disability status. Further, given the relative brevity of administering measures of perceived WA and invasiveness of the WAI (requiring information about specific and personal medial conditions), we believe that organizational researchers are better served using measures of perceived WA for most purposes.

Our meta-analytic analysis of the single item "current WA compared to your lifetime best" demonstrated surprisingly consistent relations among important correlates of interest (e.g., job demands, job resources, health). Although, there were also some notable differences, such as psychosocial personal resources being more strongly related to longer measures of perceived WA, this finding suggests that the "current WA" item is quite important for measures of perceived WA. Indeed, the item analysis from Study 2 showed that while three WA items - current, mental, and interpersonal - were significantly related to the correlates of WA, the physical WA item showed the weakest pattern and was uncorrelated with the outcomes of organizational commitment and turnover intentions. Given that Study 2 consisted of relatively few workers in primarily physically demanding occupations, the item "WA in relation to the physical demands of you job" may not map well onto the study sample, and thus may be less valuable in that context.

Taken together, although we view the measurement of WA as an area ripe for future research, one measure of perceived WA that we view as promising is the four-item measure adapted from the WAI by McGonagle and colleagues (2014, 2015). Previous studies of perceived WA have considered current WA compared to lifetime best, current 
WA relative to specific demands (e.g., mental, physical), and anticipated future WA. However, based on the conceptual definition we have provided, we argue that focusing on perceptions of current ability with respect to occupational demands is the most theoretically sound approach to assessing WA. Thus, to properly assess WA among employees across a range of occupations, the four item measure from McGonagle and colleagues (2015) may be ideal as it taps overall current WA, and a range of specific demands. On the other hand, modifying the measure to be more targeted, focusing on overall and physical WA may be best for jobs that involve overwhelmingly physical occupations (e.g., construction workers, trade jobs), and dropping the physical demands item may be warranted in cases where very few physical demands are present. Occupation Type as a Moderator

Researchers have largely viewed certain occupations as a risk factor for poor WA (Ilmarinen et al., 1991A, 1997), and we expanded on this research by investigating the moderating role of occupation type. Although occupation type did not moderate the relations between WA and job demands, age, or job outcomes, occupation type did serve as a moderator for several other important correlates. Specifically, mental health, job control, and strain were more strongly related to WA among those in white-collar occupations compared to those in blue-collar occupations. Reflecting on these findings, there appears to be support for a matching paradigm between considering the demands of occupational contexts and correlates that are more strongly related to WA within that context. That is, white-collar occupations are characterized as being primarily mentally demanding occupations, and we found evidence that mental health is a more important 
resource for white-collar workers compared to blue-collar workers. Similarly, within blue-collar occupations and nursing/healthcare following specific safety protocols may be quite important, and as such, job control may be markedly less important for WA. Similarly, strain is most often measured as a perceptual indicator and focuses on mental fatigue or burnout and, as such, may be most important when considering WA among white-collar workers compared to blue-collar workers.

Other findings regarding occupation as a moderator were less straightforward. Specifically, physical health was less strongly related to WA among samples of bluecollar workers. This finding contradicts a matching paradigm. That is, physical health should be a resource for blue-collar workers to meet the physical demands of their job. When comparing healthcare workers to those in white collar and blue collar occupations we also found interesting results. Mental health was more strongly related to WA among white-collar workers, and there were marginally significant differences in the relations between BMI, pain symptoms, and physical activity, such that each were somewhat more strongly related to WA among white collar workers compared to nursing/healthcare workers. Further, physical activity and refraining from smoking were more strongly related to WA among those in blue-collar occupations than those in nursing/healthcare. Again, in some ways these findings are surprising. Healthcare is characterized as being particularly demanding, often encompassing long shifts and high levels of mental, physical, and emotional demands. Thus, high levels of mental health, engaging in positive health behaviors, lower BMI, and not having pain symptoms should be extremely important for healthcare workers. To explain these noted discrepancies, we 
posit that workers within blue-collar occupations and those in healthcare may be more similar to one another - not necessarily due to the occupation per se but due to selfselection and attrition. That is, if workers in either field are unable to meet the demands of their job, physical demands for blue-collar workers, and overall taxing working conditions for healthcare, they may exit the workforce entirely or shift their careers to find a better fit with their abilities. Indeed, nursing in particular has struggled to retain nurses due to the high-demand nature of the job (Buerhaus, Donelan, Ulrich, Norman, \& Dittus, 2006), an issue even more important to consider for younger nurses (Symes et al., 2005). Taken together, this pattern of results suggests the need to work further upstream, by taking active steps to promote WA earlier in workers' careers and rehabilitate those experiencing low WA, steps which will be increasingly important as the workforce ages, and for specific occupations (e.g., nurses, trade workers).

Perceived Work Ability and Related Constructs

In addition to synthesizing the WA literature, we have also provided evidence of the predictive validity of WA and the unique value of WA in relation to the perceptual constructs of perceived fit, general self-efficacy, and job self-efficacy. We found in our meta-analyses that perceived WA predicted future disability status, albeit to a lesser extent that the WAI, and that measures of perceived WA predicted other exit behaviors, strain, job satisfaction, and performance in a similar manner to the WAI. The results of Study 2 further demonstrated that perceived WA can also explain unique variance in important personal and organizational variables. Specifically, perceived WA explained unique variance beyond self-efficacy and perceived fit in general, mental, physical, and 
relative health, as well as engagement, strain, task performance, OCBIs, and OCBOs. Taken together these findings further demonstrate two key ideas. First, WA, even when assessed via perceived WA, is distinct from the aforementioned related constructs. Second, despite being conceptually related to the established constructs of self-efficacy and perceived fit, the results of Study 2 showed that perceived WA is important to consider in its own right and can provide unique value in explaining variance in a number of covariates beyond that of established perceptual variables.

Practical Implications

This study highlights the importance of WA to outcomes valued by society, organizations, and workers. Accordingly, it suggests potential leverage points for organizations to help support the WA of their workforce. These include reducing demands, increasing resources, and promoting individual behavior change efforts (e.g., exercising, smoking cessation). This study demonstrates that although in many cases the resources and demands to address are consistent across occupational groups, there are a number of areas where targeted approaches may be most influential. For example, job control is in many ways hailed as a panacea for promoting worker wellbeing, but demonstrated only modest relations with WA among blue-collar occupations and was more important for WA among white-collar workers than those in nursing and healthcare. Further, the results of this study point to WA as an important focal variable for interventions aimed at improving worker wellbeing, performance, and successful aging. Indeed, interventions to promote WA often focus on health-based factors (Pohjonen \& Ranta, 2001; Rutanen et al., 2014), but have begun to include more psychosocial factors 
(Elo, Ervasti, Kuosma, \& Mattila, 2008; Müller, Weigl, Heiden, Glaser, \& Angerer, 2012), a trend that is supported by our research and for which the present study identifies some specific points of leverage outside of commonly studied strategies (e.g., promoting fairness, non-work social support, sleep, focusing on mental health, and SOC behaviors).

The present research also demonstrates that relatively short and simple measures of WA can be valuable to employers as a diagnostic tool to take the "pulse" of their employees' ability to meet job demands. That is, perceived WA can explain unique variance in important organizational constructs (e.g., engagement, health, performance, intentions to apply for disability). Further, generally speaking, assessing perceived WA requires fewer items (e.g., 4 items; McGonagle et al., 2015) than established measures such as of perceived fit (9 items; Cable \& DeRue, 2002), general self-efficacy (8 items; Chen et al., 2003), or job self-efficacy (6 items; Rigotti et al., 2008; or 8 items; Chen, Goddard, \& Casper, 2004), further supporting the utility of perceived WA in organizational research.

Finally, given WA's predictive power, from a societal perspective monitoring the WA of the workforce could provide an economic advantage to countries and societies wishing to maintain a high-functioning workforce, while simultaneously increasing wellbeing and reducing medical, disability, and retirement costs. Indeed, as industrialized workforces age, promoting WA will be an increasingly important focus for organizations, but also for the wellbeing of societal systems (e.g., retirement and healthcare systems). In other words, the utilization and tracking of WA across multiple levels of analysis can 
have a significant impact on the workforce at the individual, organizational, and societal levels, for example as an outcome variable to be monitored following policy changes. Limitations and Future Directions

Although this study makes several important contributions, it does have some limitations. First, particularly when drawing on literature from the field of occupational medicine, some measures of WA and its correlates used relatively weak measures (e.g., single-item indicators). These measures are frequently used in the medical literature, which often categorizes data in ways that lose information (e.g., creating categories from continuous scales). However, despite these limitations, we still found strong support for nearly all of our hypothesized direct relationships, and in several cases, we identified moderators. Further, the relatively weak measures used in past research may in fact underestimate the observed relations relative to the true relations among WA and its correlates.

Second, our meta-analytic investigation was sometimes limited by the available data. As such, there are several variables that may be related to WA and are important for organizations, but we were unable to assess them because there were an insufficient number of studies that did so. Two notable examples stood out to us. First, personal resources were identified as among the most strongly related to WA. However, a large number of personality variables have not been assessed in relation to WA (e.g., proactivity, adaptability, openness, agreeableness, extraversion, and emotional intelligence). Second, although we were able to establish a relationship between WA and job performance, additional research linking WA to non-self-report measures of job 
performance, distinguishing among different types of performance (e.g., organizational citizenship behaviors, task performance, objective indicators of performance, and counterproductive work behaviors), and identifying moderators to the WA to performance relation are particularly important areas for future research.

Finally, although we have presented our findings within the JD-R theoretical framework, which implies directionality and poses the potential for moderating effects between demands and resources, our meta-analytic investigation does not test each tenet of this theory. Specifically, moderating effects were not examined between demands and resources as we did not have the primary data available to conduct such analyses. Future research should address this potential gap. In addition, this meta-analytic investigation contains studies that are both cross-sectional and longitudinal and thus does not allow us to confirm directionality. For example, it is probable that the relationship between health and WA and WA and performance may be reciprocal. Although this is a concern, it is important to note that in several cases, such as in predicting future disability claims and retirement, the effect size estimates were based entirely on longitudinal studies with WA assessed at one time point and employment status at later time point. Further, in some studies, the length of time between measurement points was more than 10 years, which is remarkable in its own right and bolsters our argument that the hypothesized effects do indeed occur in the discussed temporal order. Despite evidence suggesting the temporal manner of these relationships, we view the causal nature of these relations as a gap that warrants more research.

Conclusion 
Prior to this meta-analytic investigation, the literature on WA was lacking an integrated synthesis of the empirical research. The wide range of measures used to assess WA and the absence of a nomological network made it difficult to utilize this promising construct to its full potential. In this paper, we have identified personal and contextual factors associated with WA using the JD-R framework. We found that WA is a useful predictor not only of worker attitudes and intentions, but also of critical work and healthrelated behaviors such as turnover and disability. Although we found specific instances where the WAI measure may hold stronger predictive power than measures of perceived WA, our general finding is that significant relationships between WA and its theoretical antecedents and outcomes are present regardless of the WA measure used. Finally, we assessed the important potential moderator of occupation to determine if correlates of WA vary across occupational contexts. Taken together, these findings suggest that employers, workplace researchers, and practitioners should utilize measures of WA as a workforce management tool to anticipate future employee and organizational human resource needs, promote positive job attitudes, and enhance engagement and performance. Preventive use of WA measures can facilitate the development of interventions to maintain and enhance employees' work ability before significant problems arise. In the context of today's aging workforce and expected trend of lower workforce participation (Toossi, 2015; U.S. Bureau of Labor Statistics, 2017), this study provides the synthesis and direction needed to move this area of research forward, promoting the WA and sustained health of individuals and organizations. 
Figure 1 Theoretical Model of Work Ability

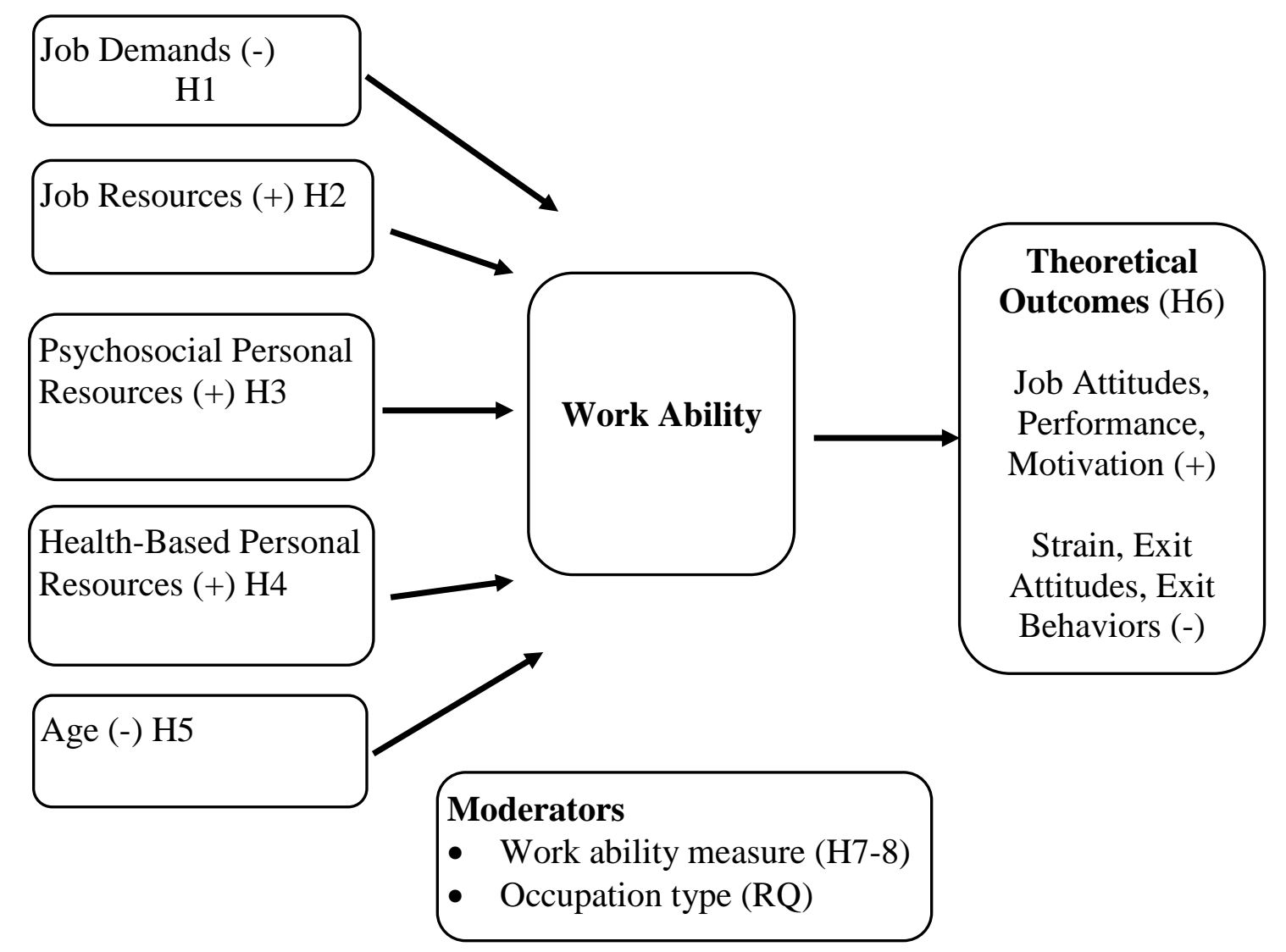

Figure 1. This model depicts the theoretical antecedents and outcomes of work ability. 
Figure 2 Results Study 1A

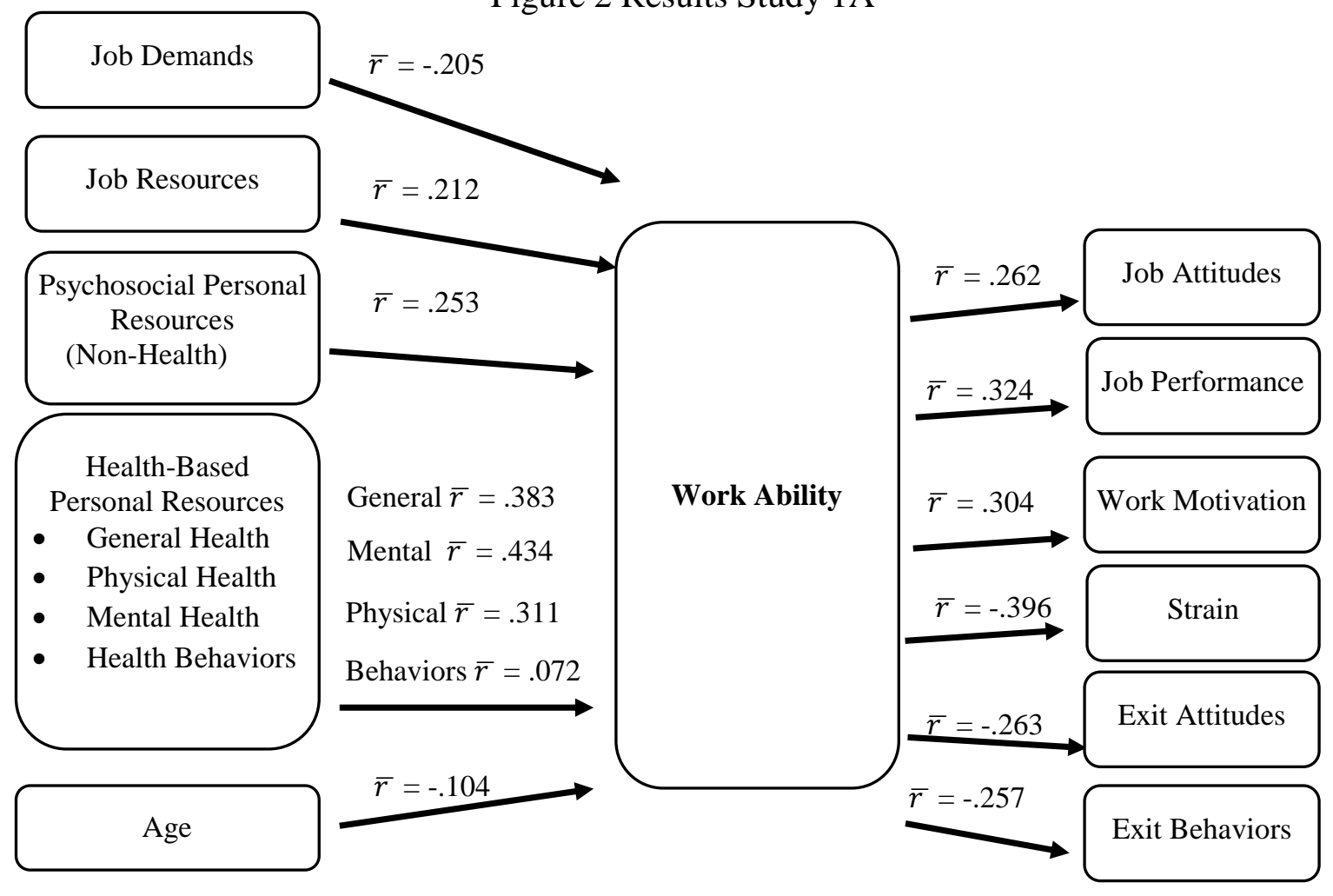

\section{Moderators}

- Work Ability Measure

- Occupation Type

Figure 2. Meta-analytic results reporting the relationships between work ability and its theoretical antecedents and outcomes. 
Table 1

Random-Effects Meta-Analytic Estimates of the Relationships between Work Ability and Its Theoretical Antecedents

\begin{tabular}{|c|c|c|c|c|c|c|c|}
\hline \multirow[b]{2}{*}{ Theoretical Antecedent } & \multirow[b]{2}{*}{$K$} & \multirow[b]{2}{*}{$N$} & \multirow[b]{2}{*}{$\bar{r}$} & \multicolumn{2}{|c|}{$95 \%$ CI $\boldsymbol{r}$} & \multirow[b]{2}{*}{$Q$} & \multirow[b]{2}{*}{$I^{2}$} \\
\hline & & & & LL & $\mathrm{UL}$ & & \\
\hline Job Demands & 55 & 52,136 & -.205 & -.247 & -.163 & $1496.71 * *$ & 96.39 \\
\hline Quantitative Demands & 28 & 29,624 & -.200 & -.246 & -.154 & $317.34 * *$ & 91.49 \\
\hline Mental/Emotional Demands & 12 & 6,402 & -.270 & -.372 & -.162 & $209.31 * *$ & 94.75 \\
\hline Physical Demands & 30 & 28,845 & -.199 & -.236 & -.161 & $294.64 * *$ & 90.16 \\
\hline Environmental Conditions & 10 & 7,620 & -.110 & -.160 & -.049 & $67.31 * *$ & 86.63 \\
\hline Workplace Mistreatment & 14 & 7,909 & -.188 & -.282 & -.090 & $225.02 * *$ & 94.22 \\
\hline Job Resources & 62 & 75,168 & .212 & .185 & .239 & $841.06^{* *}$ & 92.75 \\
\hline Coworker Support & 15 & 9,595 & .258 & .210 & .304 & $62.31 * *$ & 77.53 \\
\hline Supervisor Support & 25 & 17,830 & .225 & .185 & .265 & $169.52 * *$ & 85.84 \\
\hline Job Control & 40 & 46,614 & .218 & .186 & .249 & $443.52 * *$ & 91.21 \\
\hline Task Resources & 13 & 14,756 & .223 & .163 & .281 & $143.05^{* *}$ & 91.61 \\
\hline Rewards & 6 & 15,047 & .228 & .131 & .321 & $151.38 * *$ & 96.70 \\
\hline Justice Perceptions & 15 & 12,405 & .272 & 191 & .350 & $271.36 * *$ & 94.84 \\
\hline Org. Climate & 11 & 5,665 & .197 & .152 & .241 & $26.20 * *$ & 61.84 \\
\hline Psychosocial Personal Resources & 39 & 31,174 & .253 & .206 & .299 & $1053.94 * *$ & 96.39 \\
\hline Core Self-Evaluations & 11 & 5,032 & .377 & .294 & .455 & $84.38 * *$ & 88.15 \\
\hline Job Self-Efficacy & 6 & 1,666 & .352 & .284 & .417 & 8.17 & 38.80 \\
\hline Conscientiousness & 4 & 2,193 & .341 & .234 & .440 & $11.46^{* *}$ & 73.82 \\
\hline Positive Traits (e.g., Optimism) & 4 & 2,204 & .282 & 167 & .389 & $12.07 * *$ & 75.14 \\
\hline Cognitive Ability & 4 & 528 & .234 & .111 & .350 & $6.36 \dagger$ & 52.82 \\
\hline Coping & 3 & 9,248 & .113 & .038 & .186 & 3.87 & 48.34 \\
\hline Negative Traits (e.g., Hostility) & 7 & 3,078 & -.295 & -.362 & -.225 & $18.70^{* *}$ & 67.91 \\
\hline Grit/Resilience & 3 & 638 & .371 & .302 & .436 & .21 & .00 \\
\hline SOC Behaviors & 7 & 8,713 & .151 & .082 & .219 & $28.30 * *$ & 78.80 \\
\hline Social Support (non-work) & 8 & 5,176 & .204 & .073 & .328 & $288.96 * *$ & 97.58 \\
\hline \multicolumn{8}{|l|}{ Health-Based Personal Resources } \\
\hline General Health & 87 & 113,439 & .383 & .343 & .421 & $4136.67 * *$ & 97.92 \\
\hline Symptoms (Non-Pain) & 12 & 15,508 & -.389 & -.470 & -.301 & $306.42 * *$ & 96.41 \\
\hline Current Health Status & 32 & 56,775 & .335 & .276 & .391 & $1279.07 * *$ & 97.58 \\
\hline Previous Absenteeism ${ }^{\mathrm{a}}$ & 15 & 24,594 & -.238 & -.295 & -.180 & $158.49 * *$ & 91.17 \\
\hline Sleep & 11 & 13,348 & .282 & .220 & .342 & $74.70 * *$ & 86.61 \\
\hline Mental Health & 43 & 68,684 & .434 & .388 & .477 & $1,567.55^{* *}$ & 97.32 \\
\hline Anxiety & 6 & 4,119 & -.380 & -.452 & -.302 & $16.31 *$ & 69.34 \\
\hline Depressive symptoms & 14 & 19,338 & -.460 & -.542 & -.370 & $563.89 * *$ & 97.70 \\
\hline Physical Health & 84 & 82,421 & .311 & .266 & .354 & $3074.86 * *$ & 97.30 \\
\hline Physical Ability & 18 & 1,710 & .240 & .123 & .350 & $83.00 * *$ & 79.52 \\
\hline BMI & 31 & 37,264 & .125 & .097 & .153 & $122.10 * *$ & 75.43 \\
\hline Objective Health Indicators & 5 & 945 & .139 & -.035 & .305 & $26.23 * *$ & 84.75 \\
\hline Symptoms (Pain-Related) & 38 & 29,133 & .357 & .316 & .397 & $556.09 * *$ & 93.35 \\
\hline Health Behaviors & 41 & 50,574 & .072 & .049 & .094 & $149.06^{* *}$ & 73.17 \\
\hline Physical Activity & 29 & 15,318 & .113 & .076 & .150 & $133.01 * *$ & 78.95 \\
\hline Smoking (Tobacco) & 31 & 44,828 & -.080 & -.104 & -.056 & $101.79 * *$ & 70.53 \\
\hline
\end{tabular}


Table 1 (continued)

\begin{tabular}{|c|c|c|c|c|c|c|c|}
\hline \multirow[b]{2}{*}{ Theoretical Antecedent } & \multirow[b]{2}{*}{$K$} & \multirow[b]{2}{*}{$n$} & \multirow[b]{2}{*}{$\bar{r}$} & \multicolumn{2}{|c|}{$95 \% \mathrm{CI}$} & \multirow[b]{2}{*}{$Q$} & \multirow[b]{2}{*}{$I^{2}$} \\
\hline & & & & $\mathrm{LL}$ & UL & & \\
\hline Alcohol Consumption & 20 & 28,478 & -.007 & -.052 & .037 & $194.82 * *$ & 90.25 \\
\hline Age & 111 & 108,259 & -.114 & -.135 & -.093 & $2508.29 * *$ & 95.62 \\
\hline
\end{tabular}

Note. All analyses were conducted using random-effects models to compute the weighted average correlation $(\overline{\boldsymbol{r}})$. ** indicates $p<.01$, * indicates $p<.05$, and $\dagger$ indicates $p<.10$. ${ }^{a}$ Previous sick leave is item 5 in the WAI, however studies included in this analysis used WA measures that were either perceived WA measures, or omitted item 5 from their WA measure. In analyses where an overall estimate includes sub-analyses in the opposite direction, the effects in the opposite direction (e.g., anxiety) are recoded to represent that broader category in a positive manner (e.g., positive mental health). 
Table 2

Random-Effects Meta-Analytic Estimates of the Relationships between Work Ability and its Theoretical Outcomes

\begin{tabular}{|c|c|c|c|c|c|c|c|}
\hline \multirow[b]{2}{*}{ Theoretical Outcome } & \multirow[b]{2}{*}{$K$} & \multirow[b]{2}{*}{$n$} & \multirow[b]{2}{*}{$\overline{\boldsymbol{r}}$} & \multicolumn{2}{|c|}{$95 \%$ CI $\boldsymbol{r}$} & \multirow[b]{2}{*}{$Q$} & \multirow[b]{2}{*}{$I^{2}$} \\
\hline & & & & LL & $\mathrm{UL}$ & & \\
\hline Job Attitudes & 19 & 19,842 & .262 & .213 & .309 & $182.40 * *$ & 90.13 \\
\hline Job Satisfaction & 10 & 11,781 & 283 & .235 & .330 & $37.21 * *$ & 75.81 \\
\hline Org. Commitment & 6 & 7,084 & .256 & .152 & .353 & $80.12 * *$ & 93.76 \\
\hline Over-Commitment & 4 & 1,280 & -.218 & -.340 & -.088 & $14.77^{* * *}$ & 79.69 \\
\hline Job Performance (Self-Rated) & 9 & 24,524 & .324 & .239 & .404 & $434.12 * *$ & 98.16 \\
\hline Work Motivation & 16 & 18,241 & .304 & .225 & .379 & $397.24 * *$ & 96.22 \\
\hline Strain & 39 & 23,909 & -.396 & -.456 & -.333 & $1480.30 * *$ & 94.43 \\
\hline Fatigue & 17 & 9,211 & -.416 & -.482 & -.344 & $212.05 * *$ & 92.46 \\
\hline Burnout & 14 & 9,783 & -.444 & -.505 & -.378 & $172.76^{* *}$ & 92.48 \\
\hline Perceived Stress & 13 & 7,691 & -.347 & -.452 & -.231 & $471.64 * *$ & 97.46 \\
\hline Exit Intentions & 21 & 35,235 & -.263 & -.349 & -.173 & $580.10 * *$ & 96.52 \\
\hline Retirement Intentions & 9 & 9,060 & -.241 & -.388 & -.083 & $482.12 * *$ & 98.34 \\
\hline Turnover Intentions & 13 & 26,478 & -.273 & -.354 & -.188 & $96.87 * *$ & 87.61 \\
\hline Exit Behaviors & 20 & 44,811 & -.257 & -.324 & -.189 & $1639.07 * *$ & 98.84 \\
\hline Future Absenteeism & 13 & 30,505 & -.225 & -.305 & -.142 & $1448.57 * *$ & 99.17 \\
\hline Future Disability Status & 6 & 4,967 & -.384 & -.512 & -.239 & $150.209 * *$ & 96.67 \\
\hline Retirement & 4 & 5,598 & -.189 & -.268 & -.106 & $15.47 * *$ & 80.61 \\
\hline
\end{tabular}

Note. All analyses were conducted using random-effects models to compute the weighted average correlation $(\overline{\boldsymbol{r}})$. The Q statistic indicates heterogeneity among the effect size estimates, suggesting there are moderators to the point estimate when it is significant. ** indicates $p<.01, *$ indicates $p$ $<.05$, and $\dagger$ indicates $p<.10$. 
Table 3

Mixed-Effects Meta-Analytic Estimates of the Relationships Between WA and its Antecedents (by WA Measure)

\begin{tabular}{|c|c|c|c|c|c|c|c|}
\hline \multirow[b]{2}{*}{ Theoretical Antecedent } & \multirow[b]{2}{*}{$\begin{array}{c}\text { WA } \\
\text { Measure }\end{array}$} & \multirow[b]{2}{*}{$K$} & \multirow[b]{2}{*}{$n$} & \multirow[b]{2}{*}{$\bar{r}$} & \multicolumn{2}{|c|}{$95 \%$ CI $\overline{\boldsymbol{r}}$} & \multirow[b]{2}{*}{$Q$} \\
\hline & & & & & $\mathrm{LL}$ & UL & \\
\hline \multirow[t]{2}{*}{ Job Demands } & WAI & 27 & 12,013 & -.207 & -.268 & -.145 & .01 \\
\hline & PWA & 29 & 40,526 & -.204 & -.265 & -.144 & \\
\hline \multirow[t]{2}{*}{ Quantitative Demands } & WAI & 11 & 3,300 & -.215 & -.291 & -.136 & .21 \\
\hline & PWA & 17 & 26,320 & -.192 & -.251 & -.133 & \\
\hline Mental/Emotional & WAI & 4 & 830 & -.223 & -.345 & -.094 & 1.75 \\
\hline Demands & PWA & 8 & 5,572 & -.368 & -.524 & -.188 & \\
\hline \multirow[t]{2}{*}{ Physical Demands } & WAI & 13 & 7,286 & -.163 & -.225 & -.101 & 1.97 \\
\hline & PWA & 18 & 21,957 & -.222 & -.274 & -.168 & \\
\hline Environmental & WAI & 4 & 2,098 & -.111 & -.195 & -.025 & .00 \\
\hline Conditions & PWA & 6 & 5,522 & -.109 & -.175 & -.043 & \\
\hline Workplace & WAI & 5 & 2,121 & -.284 & -.451 & -.099 & $3.10 \dagger$ \\
\hline Mistreatment & PWA & 9 & 5,788 & -.134 & -.226 & -.039 & \\
\hline \multirow[t]{2}{*}{ Job Resources } & WAI & 26 & 25,094 & .245 & .201 & .288 & $3.90 *$ \\
\hline & PWA & 39 & 52,541 & .188 & .150 & .224 & \\
\hline \multirow[t]{2}{*}{ Coworker Support } & WAI & 4 & 1,053 & .332 & .235 & .422 & $2.98 \dagger$ \\
\hline & PWA & 11 & 8,542 & .235 & .181 & .288 & \\
\hline \multirow[t]{2}{*}{ Supervisor Support } & WAI & 6 & 3,986 & .291 & .215 & .363 & $3.62 \dagger$ \\
\hline & PWA & 21 & 15,404 & .207 & .163 & .249 & \\
\hline \multirow[t]{2}{*}{ Job Control } & WAI & 14 & 8,159 & .232 & .173 & .289 & .66 \\
\hline & PWA & 27 & 39,363 & .202 & .159 & .245 & \\
\hline \multirow[t]{2}{*}{ Task Resources } & WAI & 6 & 6,906 & .209 & .136 & .280 & .39 \\
\hline & PWA & 8 & 8,253 & .239 & .177 & .299 & \\
\hline \multirow[t]{2}{*}{ Justice Perceptions } & WAI & 10 & 5,654 & .316 & .212 & .413 & 2.04 \\
\hline & PWA & 5 & 6,751 & .186 & .033 & .330 & \\
\hline \multirow[t]{2}{*}{ Org. Climate } & WAI & 3 & 1,566 & .237 & .153 & .317 & 1.33 \\
\hline & PWA & 8 & 4,099 & .180 & .128 & .231 & \\
\hline Psychosocial Personal & WAI & 17 & 7,967 & .217 & .142 & .288 & 1.43 \\
\hline Resources & PWA & 24 & 24,330 & .275 & .213 & .334 & \\
\hline \multirow[t]{2}{*}{ Core Self-Evaluations } & WAI & 4 & 768 & .367 & .220 & .498 & .06 \\
\hline & PWA & 8 & 4,480 & .388 & .291 & .477 & \\
\hline Negative Traits & WAI & 3 & 552 & -.199 & -.314 & -.079 & $4.19 *$ \\
\hline (e.g., Hostility) & PWA & 4 & 2,526 & -.346 & -.423 & -.264 & \\
\hline
\end{tabular}


Table 3 (continued)

\begin{tabular}{|c|c|c|c|c|c|c|c|}
\hline \multirow[b]{2}{*}{ Theoretical Antecedent } & \multirow[b]{2}{*}{$\begin{array}{c}\text { WA } \\
\text { Measure }\end{array}$} & \multirow[b]{2}{*}{$K$} & \multirow[b]{2}{*}{$n$} & \multirow[b]{2}{*}{$\bar{r}$} & \multicolumn{2}{|c|}{$95 \%$ CI $\bar{r}$} & \multirow[b]{2}{*}{$Q$} \\
\hline & & & & & LL & UL & \\
\hline \multicolumn{8}{|l|}{$\begin{array}{l}\text { Health-Based Personal } \\
\text { Resources }\end{array}$} \\
\hline \multirow[t]{2}{*}{ General Health } & WAI & 39 & 42,741 & .433 & .375 & .487 & $4.77 *$ \\
\hline & PWA & 52 & 84,455 & .347 & .294 & .398 & \\
\hline \multirow[t]{2}{*}{ Symptoms (Non-Pain) } & WAI & 5 & 5,203 & -.417 & -.538 & -.280 & .37 \\
\hline & PWA & 7 & 10,305 & -.365 & -.478 & -.239 & \\
\hline \multirow[t]{2}{*}{ Current Health Status } & WAI & 10 & 17,391 & .476 & .387 & .556 & $11.14^{* *}$ \\
\hline & PWA & 25 & 52,738 & .290 & .227 & .350 & \\
\hline \multirow[t]{2}{*}{ Sleep } & WAI & 9 & 13,276 & .279 & .215 & .342 & .79 \\
\hline & PWA & 3 & 475 & .352 & .201 & .487 & \\
\hline \multirow[t]{2}{*}{ Physical Health } & WAI & 57 & 42,441 & .333 & .280 & .384 & 2.52 \\
\hline & PWA & 31 & 54,186 & .262 & .188 & .332 & \\
\hline \multirow[t]{2}{*}{ Physical Ability } & WAI & 15 & 1,538 & .253 & .128 & .371 & .32 \\
\hline & PWA & 4 & 253 & .176 & -.072 & .403 & \\
\hline \multirow[t]{2}{*}{ BMI } & WAI & 21 & 26,714 & .137 & .102 & .172 & 1.55 \\
\hline & PWA & 12 & 23,792 & .102 & .059 & .144 & \\
\hline \multirow{2}{*}{$\begin{array}{l}\text { Symptoms } \\
\text { (Pain-Related) }\end{array}$} & WAI & 20 & 21,222 & .385 & .333 & .434 & 2.59 \\
\hline & PWA & 20 & 36,885 & .326 & .273 & .376 & \\
\hline \multirow[t]{2}{*}{ Mental Health } & WAI & 27 & 28,420 & .452 & .398 & .502 & 1.43 \\
\hline & PWA & 17 & 53,119 & .399 & .328 & .466 & \\
\hline \multirow[t]{2}{*}{ Depressive symptoms } & WAI & 6 & 4,738 & .467 & -.596 & .315 & .02 \\
\hline & PWA & 8 & 14,615 & .455 & -.568 & .326 & \\
\hline \multirow[t]{2}{*}{ Health Behaviors } & WAI & 29 & 31,239 & .074 & .046 & .103 & .02 \\
\hline & PWA & 16 & 32,775 & .078 & .041 & .114 & \\
\hline \multirow[t]{2}{*}{ Physical Activity } & WAI & 20 & 8,615 & .110 & .065 & .156 & .55 \\
\hline & PWA & 12 & 7,304 & .138 & .081 & .194 & \\
\hline \multirow[t]{2}{*}{ Smoking (Tobacco) } & WAI & 23 & 28,906 & -.074 & -.103 & -.045 & .37 \\
\hline & PWA & 10 & 29,164 & -.090 & -.132 & -.048 & \\
\hline \multirow[t]{2}{*}{ Alcohol Consumption } & WAI & 13 & 11,996 & -.017 & -.075 & .042 & .10 \\
\hline & PWA & 8 & 16,885 & -.002 & -.072 & .068 & \\
\hline \multirow[t]{2}{*}{ Age } & WAI & 71 & 65,763 & -.129 & -.155 & -.103 & 2.13 \\
\hline & PWA & 45 & 58,731 & -.099 & -.130 & -.067 & \\
\hline
\end{tabular}


Table 3 (continued)

Note. $* *$ indicates $p<.01, *$ indicates $p<.05$, and $\dagger$ indicates $p<.10$. All analyses were conducted using mixed-effects models to compute the weighted average correlation $(\bar{r}) \cdot Q=$ represents the significance test for heterogeneity in the effect size estimates between the two subgroups (the full WAI compared to perceived WA). $K$ within these analyses may exceed the $k$ for the overall estimate because we separated studies that measured WA using the WAI and a measure of perceived WA into independent effects for these analyses. 
Table 4

Mixed-Effects Meta-Analytic Estimates of the Relationships Between WA and its Outcomes (by WA Measure)

\begin{tabular}{|c|c|c|c|c|c|c|c|}
\hline \multirow[b]{2}{*}{ Theoretical Outcome } & \multirow[b]{2}{*}{ WA Measure } & \multirow[b]{2}{*}{$K$} & \multirow[b]{2}{*}{$n$} & \multirow[b]{2}{*}{$\bar{r}$} & \multicolumn{2}{|c|}{$95 \%$ CI $\bar{r}$} & \multirow[b]{2}{*}{$Q$} \\
\hline & & & & & $\mathrm{LL}$ & UL & \\
\hline \multirow[t]{2}{*}{ Job Attitudes } & WAI & 12 & 10,414 & .235 & .180 & .288 & 2.46 \\
\hline & PWA & 8 & 9,379 & .299 & .239 & .356 & \\
\hline \multirow[t]{2}{*}{ Job Satisfaction } & WAI & 6 & 8,410 & .268 & .205 & .32 & .00 \\
\hline & PWA & 5 & 3,587 & .271 & .205 & .334 & \\
\hline \multirow[t]{2}{*}{ Strain } & WAI & 21 & 10,062 & -.414 & -.497 & -.324 & .69 \\
\hline & PWA & 19 & 14,754 & -.358 & -.453 & -.254 & \\
\hline \multirow[t]{2}{*}{ Fatigue } & WAI & 12 & 4,877 & -.441 & -.52 & -.355 & 1.27 \\
\hline & PWA & 5 & 4,334 & -.345 & -.485 & -.187 & \\
\hline \multirow[t]{2}{*}{ Burnout } & WAI & 5 & 3,678 & -.464 & -.553 & -.364 & .24 \\
\hline & PWA & 9 & 6,105 & -.431 & -.510 & -.345 & \\
\hline \multirow[t]{2}{*}{ Perceived Stress } & WAI & 6 & 3,202 & -.347 & -.503 & -.169 & .11 \\
\hline & PWA & 8 & 5,396 & -.308 & -.451 & -.149 & \\
\hline \multirow[t]{2}{*}{ Work Motivation } & WAI & 8 & 10,536 & .316 & .207 & .417 & .022 \\
\hline & PWA & 9 & 8,109 & .305 & .203 & .401 & \\
\hline \multirow[t]{2}{*}{ Exit Intentions } & WAI & 15 & 27,769 & -.313 & -.406 & -.213 & $3.35 \dagger$ \\
\hline & PWA & 6 & 7,466 & -.139 & -.296 & .026 & \\
\hline \multirow[t]{2}{*}{ Exit Behaviors } & WAI & 15 & 36,810 & -.280 & -.360 & -.196 & .00 \\
\hline & PWA & 7 & 10,504 & -.284 & -.398 & -.161 & \\
\hline \multirow{2}{*}{$\begin{array}{l}\text { Future Disability } \\
\text { Status }\end{array}$} & WAI & 4 & 3,349 & -.534 & -.657 & -.384 & $5.56^{*}$ \\
\hline & PWA & 3 & 2,263 & -.236 & -.45 & -.016 & \\
\hline \multirow[t]{2}{*}{ Future Absenteeism } & WAI & 9 & 24,065 & -.197 & -.286 & -.104 & 1.35 \\
\hline & PWA & 5 & 8,226 & -.285 & -.398 & -.164 & \\
\hline
\end{tabular}

Note. $* *$ indicates $p<.01, *$ indicates $p<.05$, and $\dagger$ indicates $p<.10$. All analyses were conducted using mixed-effects models to compute the weighted average correlation $(\bar{r}) \cdot Q=$ represents the significance test for heterogeneity in the effect size estimates between the two subgroups (the full WAI compared to perceived WA). $K$ within these analyses may exceed the $k$ for the overall estimate because we separated studies that measured WA using the WAI and a measure of perceived WA into independent effects for these analyses. 
Table 5

Mixed-Effects Meta-Analytic Estimates of the Relationships Between WA and its Antecedents and Outcomes (by Occupation)

\begin{tabular}{|c|c|c|c|c|c|c|c|c|}
\hline \multirow[b]{2}{*}{ Theoretical Antecedent } & \multirow[b]{2}{*}{$K$} & \multirow[b]{2}{*}{$n$} & \multirow[b]{2}{*}{$\bar{r}$} & \multicolumn{2}{|c|}{$95 \%$ CI $\bar{r}$} & \multirow{2}{*}{$\begin{array}{c}\text { White vs } \\
\text { Blue } \\
\text { Collar }\end{array}$} & \multirow{2}{*}{$\begin{array}{c}\begin{array}{c}\text { White Collar } \\
\text { vs Nursing }\end{array} \\
Q\end{array}$} & \multirow{2}{*}{$\begin{array}{c}\begin{array}{c}\text { Blue Collar vs } \\
\text { Nursing }\end{array} \\
Q\end{array}$} \\
\hline & & & & LL & UL & & & \\
\hline Job Demands & & & & & & 1.95 & .14 & 1.30 \\
\hline White Collar & 8 & 10,990 & -.277 & -.384 & -.164 & & & \\
\hline Blue Collar & 8 & 3,359 & -.161 & -.278 & -.039 & & & \\
\hline Nursing/Healthcare & 9 & 3,883 & -.244 & -.363 & -.118 & & & \\
\hline Physical Job & & & & & & .27 & .34 & .00 \\
\hline \multicolumn{9}{|l|}{ Demands } \\
\hline White Collar & 4 & 3,741 & -.215 & -.323 & -.100 & & & \\
\hline Blue Collar & 4 & 1,645 & -.179 & -.295 & -.057 & & & \\
\hline Nursing/Healthcare & 4 & 1,782 & -.189 & -.315 & -.056 & & & \\
\hline \multicolumn{9}{|l|}{ Quantitative Job } \\
\hline Demands & & & & & & & & .01 \\
\hline Blue Collar & 4 & 1,411 & -.200 & -.322 & -.071 & & & \\
\hline Nursing/Healthcare & 6 & 2,411 & -.193 & -.252 & -.134 & & & \\
\hline \multicolumn{9}{|l|}{ Workplace } \\
\hline Mistreatment & & & & & & & .52 & \\
\hline White Collar & 4 & 2,506 & -.215 & -.292 & -.136 & & & \\
\hline Nursing/Healthcare & 3 & 1,697 & -.310 & -.555 & -.016 & & & \\
\hline Job Resources & & & & & & $7.72 * *$ & $2.93 \dagger$ & .500 \\
\hline White Collar & 13 & 15,037 & .273 & .220 & .324 & & & \\
\hline Blue Collar & 10 & 3,004 & .172 & .099 & .244 & & & \\
\hline Nursing/Healthcare & 13 & 13,403 & .210 & .151 & .269 & & & \\
\hline Job Control & & & & & & $13.24 * *$ & $5.56^{*}$ & .99 \\
\hline White Collar & 10 & 13,141 & .298 & .252 & .342 & & & \\
\hline Blue Collar & 7 & 2,442 & .140 & .069 & .210 & & & \\
\hline Nursing/Healthcare & 6 & 1,421 & .200 & .122 & .277 & & & \\
\hline Justice Perceptions & & & & & & & & .095 \\
\hline Blue Collar & 4 & 1,085 & .235 & .079 & .381 & & & \\
\hline Nursing/Healthcare & 5 & 3,888 & .203 & .073 & .326 & & & \\
\hline Org. Climate & & & & & & & $.1 J$ & \\
\hline White Collar & 3 & 2,856 & .203 & .151 & .254 & & & \\
\hline Nursing/Healthcare & 3 & 260 & .228 & .102 & .348 & & & \\
\hline Psychosocial Personal & & & & & & 1.38 & .01 & .52 \\
\hline Resources & & & & & & & & \\
\hline White Collar & 7 & 3,527 & .243 & .140 & .341 & & & \\
\hline Blue Collar & 4 & 1,436 & .165 & .020 & .303 & & & \\
\hline Nursing/Healthcare & 8 & 3,116 & .238 & .136 & .336 & & & \\
\hline
\end{tabular}


Table 5 (continued)

\begin{tabular}{|c|c|c|c|c|c|c|c|c|}
\hline \multirow[b]{2}{*}{ Theoretical Antecedent } & \multirow[b]{2}{*}{$K$} & \multirow[b]{2}{*}{$n$} & \multirow[b]{2}{*}{$\bar{r}$} & \multicolumn{2}{|c|}{$95 \%$ CI $\bar{r}$} & \multirow{2}{*}{$\begin{array}{c}\text { White vs } \\
\text { Blue Collar } \\
Q\end{array}$} & \multirow{2}{*}{$\begin{array}{c}\text { White Collar } \\
\text { vs Nursing } \\
\end{array}$} & \multirow{2}{*}{$\begin{array}{c}\text { Blue Collar vs } \\
\text { Nursing } \\
Q\end{array}$} \\
\hline & & & & LL & $\mathrm{UL}$ & & & \\
\hline General Health & & & & & & $2.87 \dagger$ & .028 & $3.37 \dagger$ \\
\hline White Collar & 12 & 15,395 & .447 & .351 & .533 & & & \\
\hline Blue Collar & 14 & 9,456 & .349 & .251 & .439 & & & \\
\hline Nurses/Healthcare & 12 & 14,692 & .457 & .359 & .545 & & & \\
\hline Current Health Status & & & & & & 1.15 & .002 & .44 \\
\hline White Collar & 3 & 1,484 & .422 & .241 & .575 & & & \\
\hline Blue Collar & 3 & 1,905 & .362 & .171 & .526 & & & \\
\hline Nursing/Healthcare & 3 & 1,134 & .425 & .232 & .587 & & & \\
\hline Mental Health & & & & & & $7.99 * *$ & $7.14 * *$ & .85 \\
\hline White Collar & 9 & 12,733 & .529 & .462 & .590 & & & \\
\hline Blue Collar & 6 & 1,687 & .400 & .296 & .494 & & & \\
\hline Nurses/Healthcare & 5 & 3,241 & .396 & .282 & .500 & & & \\
\hline Physical Health & & & & & & & & \\
\hline White Collar & 9 & 5,660 & .512 & .413 & .599 & $6.33 *$ & 2.33 & .87 \\
\hline Blue Collar & 20 & 7,354 & .262 & .175 & .345 & & & \\
\hline Nursing/Healthcare & 12 & 6,850 & .330 & .227 & .427 & & & \\
\hline BMI & & & & & & .64 & $3.62 \dagger$ & .04 \\
\hline White Collar & 3 & 1,345 & .161 & .071 & .249 & & & \\
\hline Blue Collar & 12 & 4,718 & .115 & .065 & .164 & & & \\
\hline Nursing/Healthcare & 3 & 1,716 & .100 & .011 & .188 & & & \\
\hline Symptoms (Pain- & & & & & & .03 & $2.88 \dagger$ & 2.26 \\
\hline Related) & & & & & & & & \\
\hline White Collar & 5 & 2,910 & .456 & .375 & .530 & & & \\
\hline Blue Collar & 6 & 2,469 & .455 & .372 & .530 & & & \\
\hline Nursing/Healthcare & 7 & 2,388 & .351 & .266 & .430 & & & \\
\hline Health Behaviors & & & & & & .86 & 1.57 & $5.98 *$ \\
\hline White Collar & 4 & 3,171 & .065 & .000 & .129 & & & \\
\hline Blue Collar & 13 & 6,108 & .109 & .065 & .152 & & & \\
\hline Nursing/Healthcare & 4 & 2,105 & -.0111 & -.080 & .057 & & & \\
\hline Physical Activity & & & & & & .01 & $3.138 \dagger$ & $5.21 *$ \\
\hline White Collar & 3 & 1,739 & .160 & .061 & .256 & & & \\
\hline Blue Collar & 8 & 5,030 & .154 & .090 & .217 & & & \\
\hline Nursing/Healthcare & 3 & 1,651 & -.008 & -.108 & .092 & & & \\
\hline Smoking & & & & & & .00 & .89 & $5.72 *$ \\
\hline White Collar & 3 & 1,476 & -.089 & -.167 & -.010 & & & \\
\hline Blue Collar & 9 & 4,142 & -.108 & -.155 & -.060 & & & \\
\hline Nursing/Healthcare & 3 & 1,607 & -.022 & -.097 & .053 & & & \\
\hline
\end{tabular}


Table 5 (continued)

\begin{tabular}{|c|c|c|c|c|c|c|c|c|}
\hline \multirow[b]{2}{*}{ Theoretical Antecedent } & \multirow[b]{2}{*}{$K$} & \multirow[b]{2}{*}{$n$} & \multirow[b]{2}{*}{$\bar{r}$} & \multicolumn{2}{|c|}{$95 \%$ CI $\bar{r}$} & \multirow{2}{*}{$\begin{array}{c}\text { White vs } \\
\text { Blue Collar } \\
Q\end{array}$} & \multirow{2}{*}{$\begin{array}{c}\text { White Collar } \\
\text { vs Nursing } \\
Q\end{array}$} & \multirow{2}{*}{$\begin{array}{c}\text { lue Collar vs } \\
\text { Nursing } \\
Q\end{array}$} \\
\hline & & & & LL & $\mathrm{UL}$ & & & \\
\hline Drinking & & & & & & .04 & & \\
\hline White Collar & 3 & 1,766 & .016 & -.102 & .134 & & & \\
\hline Blue Collar & 5 & 2,406 & -.023 & -.128 & .083 & & & \\
\hline Age & & & & & & .14 & .03 & .25 \\
\hline White Collar & 12 & 12,378 & -.132 & -.204 & -.058 & & & \\
\hline Blue Collar & 29 & 19,071 & -.114 & -.163 & -.065 & & & \\
\hline Nursing/Healthcare & 20 & 14,394 & -.134 & -.190 & -.077 & & & \\
\hline
\end{tabular}


Table 6

Mixed-Effects Meta-Analytic Estimates of the Relationships Between WA and its Antecedents and Outcomes (by Occupation)

\begin{tabular}{|c|c|c|c|c|c|c|c|c|}
\hline \multirow[b]{2}{*}{ Theoretical Outcome } & \multirow[b]{2}{*}{$K$} & \multirow[b]{2}{*}{$n$} & \multirow[b]{2}{*}{$\bar{r}$} & \multicolumn{2}{|c|}{$95 \%$ CI $\bar{r}$} & \multirow{2}{*}{$\begin{array}{c}\begin{array}{c}\text { White vs } \\
\text { Blue Collar }\end{array} \\
Q\end{array}$} & \multirow{2}{*}{$\begin{array}{c}\begin{array}{c}\text { White Collar } \\
\text { vs Nursing }\end{array} \\
Q \\
\end{array}$} & \multirow{2}{*}{$\begin{array}{c}\text { Blue Collar vs } \\
\text { Nursing }\end{array}$} \\
\hline & & & & LL & UL & & & \\
\hline Job Attitudes & & & & & & .07 & .58 & .24 \\
\hline White Collar & 5 & 3,342 & .262 & .173 & .347 & & & \\
\hline Blue Collar & 4 & 675 & .242 & .122 & .356 & & & \\
\hline Nursing/Healthcare & 3 & 8,318 & .216 & .105 & .321 & & & \\
\hline Strain & & & & & & $4.54^{*}$ & .38 & 1.19 \\
\hline White Collar & 8 & 7,237 & -.481 & -.569 & -.382 & & & \\
\hline Blue Collar & 10 & 3,365 & -.309 & -.407 & -.204 & & & \\
\hline Nursing/Healthcare & 6 & 3,230 & -.431 & -.542 & -.307 & & & \\
\hline Fatigue & & & & & & & & .94 \\
\hline Blue Collar & 3 & 670 & -.339 & -.547 & -.093 & & & \\
\hline Nursing/Healthcare & 4 & 1,097 & -.482 & -.639 & -.286 & & & \\
\hline Motivation & & & & & & $3.49 \dagger$ & & \\
\hline White Collar & 6 & 3,871 & .220 & .066 & .364 & & & \\
\hline Blue Collar & 3 & 1,647 & .437 & .244 & .597 & & & \\
\hline Exit Behaviors & & & & & & .90 & & \\
\hline White Collar & 5 & 3,189 & -.271 & -.424 & -.103 & & & \\
\hline Blue Collar & 5 & 7,318 & -.310 & -.458 & -.145 & & & \\
\hline Future Disability & & & & & & .29 & & \\
\hline White Collar & 3 & 1,738 & -.324 & -.498 & -.124 & & & \\
\hline Blue Collar & 3 & 1,320 & -.362 & -.530 & -.167 & & & \\
\hline Exit Intentions & & & & & & & 2.29 & \\
\hline White Collar & 4 & 2,817 & -.308 & -.482 & -.111 & & & \\
\hline Nursing/Healthcare & 10 & 24,495 & -.312 & -.476 & -.126 & & & \\
\hline
\end{tabular}

Note. $* *$ indicates $p<.01$, * indicates $p<.05$, and $\dagger$ indicates $p<.10$. Only comparisons where at least two occupation groups had 3 or more effect size estimates were assessed. Where the $Q$ value are presented, $Q$ indicates the heterogeneity of the effect size estimates between those two occupational groups. For example, the first column reporting a $Q$ value indicates the difference in effect size estimates between studies using white collar vs blue collar employees. 
Table 7

Zero-Order Correlations among Study 2 Variables.

\begin{tabular}{|c|c|c|c|c|c|c|c|c|c|c|c|c|c|c|c|c|c|c|c|c|c|c|c|}
\hline & & $M(\mathrm{SD})$ & 1 & 2 & 3 & 4 & 5 & 6 & 7 & 8 & 9 & 10 & 11 & 12 & 13 & 14 & 15 & 16 & 17 & 18 & 19 & 20 & 21 \\
\hline 1 & PWA & $8.23(1.35)$ & .81 & & & & & & & & & & & & & & & & & & & & \\
\hline 2 & Current WA & $8.14(1.49)$ & .84 & - & & & & & & & & & & & & & & & & & & & \\
\hline 3 & Mental WA & $8.30(1.61)$ & .82 & .70 & - & & & & & & & & & & & & & & & & & & \\
\hline 4 & $\begin{array}{l}\text { Physical } \\
\text { WA }\end{array}$ & $8.43(1.90)$ & .77 & .53 & .43 & - & & & & & & & & & & & & & & & & & \\
\hline 5 & $\begin{array}{l}\text { Interpersonal } \\
\text { WA }\end{array}$ & $8.05(1.75)$ & .78 & .51 & .54 & .43 & - & & & & & & & & & & & & & & & & \\
\hline 6 & Perc. Fit & 5.19 (1.09) & .28 & .24 & .25 & .10 & .30 & .92 & & & & & & & & & & & & & & & \\
\hline 7 & General SE & $5.68(1.06)$ & .53 & .53 & .52 & .32 & .38 & .40 & .95 & & & & & & & & & & & & & & \\
\hline 8 & Job SE & $5.92(.81)$ & .51 & .50 & .53 & .32 & .31 & .42 & .76 & .90 & & & & & & & & & & & & & \\
\hline 9 & G. Health & $5.57(1.22)$ & .48 & .52 & .43 & .28 & .35 & .26 & .37 & .31 & - & & & & & & & & & & & & \\
\hline 10 & M. Health & $5.54(1.49)$ & .43 & .44 & .49 & .12 & .37 & .30 & .51 & .40 & .62 & - & & & & & & & & & & & \\
\hline 11 & P. Health & $5.53(1.28)$ & .44 & .48 & .36 & .27 & .33 & .20 & .29 & .23 & .87 & .56 & - & & & & & & & & & & \\
\hline 12 & R. Health & $5.51(1.40)$ & .44 & .51 & .40 & .19 & .33 & .21 & .40 & .29 & .84 & .66 & .83 & - & & & & & & & & & \\
\hline 13 & $\begin{array}{l}\text { Disability } \\
\text { Int. }\end{array}$ & $1.56(1.08)$ & -.41 & -.45 & -.32 & -.30 & -.25 & -.17 & -.25 & -.23 & -.41 & -.31 & -.43 & -.39 & .94 & & & & & & & & \\
\hline 14 & $\begin{array}{l}\text { Job } \\
\text { Satisfaction }\end{array}$ & $5.19(1.53)$ & .31 & .34 & .29 & .12 & .27 & .77 & .44 & .41 & .28 & .42 & .20 & .26 & -.17 & .94 & & & & & & & \\
\hline 15 & $\begin{array}{l}\text { Org. } \\
\text { Commit. }\end{array}$ & $4.52(1.44)$ & .22 & .21 & .18 & .05 & .29 & .74 & .30 & .29 & .19 & .28 & .17 & .22 & -.16 & .70 & .92 & & & & & & \\
\hline 16 & $\begin{array}{l}\text { Turnover } \\
\text { Int. }\end{array}$ & 3.27 (1.94) & -.18 & -.18 & -.19 & -.02 & -.21 & -.67 & -.22 & -.22 & -.15 & -.25 & -.12 & -.13 & .14 & -.72 & -.74 & .92 & & & & & \\
\hline 17 & Engagement & $5.00(1.26)$ & .39 & .40 & .35 & .12 & .33 & .69 & .51 & .44 & .35 & .45 & .29 & .35 & -.19 & .80 & .70 & -.61 & .94 & & & & \\
\hline 18 & Exhaustion & $3.43(1.22)$ & -.49 & -.46 & -.45 & -.33 & -.33 & -.47 & -.53 & -.47 & -.42 & -.51 & -.38 & -.36 & .31 & -.53 & -.43 & .40 & -.61 & .87 & & & \\
\hline 19 & Task Perf. & $6.19(.74)$ & .46 & .41 & .45 & .34 & .28 & .21 & .53 & .56 & .21 & .27 & .21 & .21 & -.22 & .20 & .10 & -.13 & .30 & -.34 & .88 & & \\
\hline 20 & OCBI & $5.49(.96)$ & .43 & .39 & .36 & .27 & .36 & .35 & .55 & .50 & .17 & .30 & .10 & .19 & -.19 & .33 & .41 & -.19 & .48 & -.30 & .42 & .88 & \\
\hline 21 & OCBO & $5.90(.81)$ & .42 & .34 & .38 & .31 & .32 & .31 & .34 & .41 & .26 & .33 & .19 & .22 & -.26 & .26 & .27 & -.23 & .36 & -.34 & .54 & .43 & .76 \\
\hline
\end{tabular}


Table 7 (continued)

Note. $\mathrm{N}=282 . p<.01$ for correlations in which $r>.15 . p<.05$ for correlations in which $r>.10$. Boldface numbers indicate internal consistency of the measure. PWA = mean from the 4-item measure of perceived WA (McGonagle et al., 2015). Current WA = current work ability compared to lifetime best. Mental WA = current work ability with respect to mental demands. Physical WA = current work ability with respect to physical demands. Interpersonal WA $=$ current work ability with respect to interpersonal demands. G. Health $=$ General health. M. Health $=$ Mental health. P. Health $=$ Physical health. R. Health = Relative health. 
Table 8

Sequential Multiple Regression Models Examining Incremental Variance Explained by Perceived WA in Relation to Correlates over and above Perceived Fit, General Self-Efficacy, and Job Self-Efficacy.

\begin{tabular}{|c|c|c|c|c|c|c|c|c|c|c|c|c|}
\hline & \multicolumn{2}{|c|}{$\begin{array}{l}\text { General } \\
\text { Health }\end{array}$} & \multicolumn{2}{|c|}{$\begin{array}{l}\text { Mental } \\
\text { Health }\end{array}$} & \multicolumn{2}{|c|}{$\begin{array}{c}\text { Physical } \\
\text { Health }\end{array}$} & \multicolumn{2}{|c|}{$\begin{array}{l}\text { Relative } \\
\text { Health }\end{array}$} & \multicolumn{2}{|c|}{ Burnout } & \multicolumn{2}{|c|}{ Dis. Intentions } \\
\hline & $\beta$ & $\Delta R^{2}$ & $\beta$ & $\Delta R^{2}$ & $\beta$ & $\Delta R^{2}$ & $\beta$ & $\Delta R^{2}$ & $\beta$ & $\Delta R^{2}$ & $\beta$ & $\Delta R^{2}$ \\
\hline $\begin{array}{l}\text { Perc. } \\
\text { Fit }\end{array}$ & $.11^{* *}$ & .07 & $.10^{* * *}$ & .09 & .08 & .04 & .05 & .04 & $-.29 * *$ & .22 & -.05 & .03 \\
\hline GSE & $.16^{* *}$ & .08 & $.39 * *$ & . 18 & .13 & .06 & $.31 * *$ & .12 & $-.27 * *$ & .14 & -.03 & .04 \\
\hline JSE & -.07 & .00 & -.05 & .00 & -.12 & .00 & -.13 & .00 & -.02 & .00 & .01 & .00 \\
\hline PWA & $.40^{* *}$ & .11 & $.23^{* *}$ & .04 & $.41^{* *}$ & .12 & $.32 * *$ & .07 & $-.20 * *$ & .05 & $-.38 * *$ & .10 \\
\hline \multirow[t]{3}{*}{$\begin{array}{l}\text { Total } \\
R^{2} \\
\end{array}$} & & .26 & & .30 & & .21 & & .24 & & .41 & & .16 \\
\hline & \multicolumn{2}{|c|}{ Engagement } & \multicolumn{2}{|c|}{$\begin{array}{c}\text { Task } \\
\text { Performance }\end{array}$} & \multicolumn{2}{|c|}{ OCBO } & \multicolumn{2}{|c|}{ OCBI } & \multicolumn{2}{|c|}{$\begin{array}{c}\text { Job } \\
\text { Satisfaction }\end{array}$} & \multicolumn{2}{|c|}{$\begin{array}{c}\text { Org. } \\
\text { Commitment }\end{array}$} \\
\hline & $\beta$ & $\Delta R^{2}$ & B & $\Delta R^{2}$ & B & $\Delta R^{2}$ & B & $\Delta R^{2}$ & $\beta$ & $\Delta R^{2}$ & $\beta$ & $\Delta R^{2}$ \\
\hline $\begin{array}{l}\text { Perc. } \\
\text { Fit }\end{array}$ & $.58^{* *}$ & .48 & -.06 & .05 & $.15^{*}$ & .10 & $.12 *$ & .12 & $.71 * *$ & .60 & $.74 * *$ & .55 \\
\hline GSE & $.26^{* *}$ & .07 & $.18^{*}$ & .23 & -.05 & .06 & $.31 * *$ & .20 & $.15^{*}$ & .02 & .04 & .00 \\
\hline JSE & .06 & .00 & $.34 * *$ & .06 & $.23^{* *}$ & .04 & .14 & .01 & -.02 & .00 & -.07 & .00 \\
\hline PWA & $.12 *$ & .01 & $.21^{* *}$ & .03 & $.29 * *$ & .06 & $.16^{* *}$ & .02 & .05 & .00 & .03 & .00 \\
\hline $\begin{array}{l}\text { Total } \\
R^{2}\end{array}$ & & .55 & & .37 & & .23 & & .35 & & .62 & & .55 \\
\hline
\end{tabular}


Chapter 3: Age Discrimination and Job Outcomes: The Mediating Role of Perceived Work Ability and Fit

The results of Study 1 indicated that fairness perceptions are positively related to PWA and that age is negatively related to PWA. Moreover, the results of Study 1 provided initial evidence that PWA is a useful construct to consider, even when considering established perceptual constructs (e.g., perceived fit, job self-efficacy). However, despite PWA frequently being studied in the context of an aging workforce, interpersonal treatment based on one's age, and specifically individual perceptions of age discrimination, had not been investigated as an antecedent to PWA or perceived fit. Given that the aging workforce has been among the primary motivations for studying PWA, it is surprising that age discrimination has not been studied more thoroughly in relation to PWA. I view this omission as a substantial gap in the PWA literature, and thus, in Study 2 I investigated the effect of age discrimination on PWA and perceived fit as well as the extent to which PWA and perceived fit serve as a mediator between age discrimination and negative outcomes.

Industrialized workforces across the world are aging (Toosi, 2015; U.S. Census Bureau, 2017), and organizations must adapt to deal with this shift to an older and more age-diverse workforce. One line or research aimed at understanding how to motivate and enable older workers to continue working is research on work ability (e.g., Ilmarinen et al., 1991, 1997; McGonagle et al., 2015; von Bonsdorff et al., 2011), which, within the organizational sciences has tended to focus on perceived work ability. Perceived work ability (PWA) refers to a person's perceptions of their ability to meet the demands of 
their job (Ilmarinen, 2009; Ilmarinen et al., 1991a). After being developed in the occupational medicine literature, research has identified a number of organizational antecedents (e.g., supervisor support; Sugimura \& Thériault, 2010; role overload; McGonagle, et al., 2015) and outcomes (e.g., retirement; Feldt, Hyvönen, Mäkikangas, Kinnunen, \& Kokko, 2009) of work ability. However, despite commonly being studied in terms of the aging workforce (Ilmarinen et al., 1991a; Feldt et al., 2009) little research has linked the treatment of older people in the workplace - either by coworkers or supervisors - to PWA (see Furunes \& Mykletun, 2010 as an exception). Unfortunately, age stereotypes are more widely accepted than other stereotypes (e.g., gender, racial; Levy \& Banaji, 2002), and these stereotypes are often quite negative. Specifically, older workers are often viewed as less competent (Cuddy et al., 2005), less able to learn new skills, more likely to turn over, and more likely to experience health problems $(\mathrm{Ng} \&$ Feldman, 2012; Posthuma \& Campion, 2009). These stereotypes serve as the root of age discrimination, which has been linked to lower levels of mental health (Yuan, 2007), job satisfaction (Marchiondo, Gonzales, \& Ran, 2015) and a desire to retire earlier (BaylSmith, \& Griffin, 2014; Snape \& Redman, 2003). Despite the negative effects of age discrimination and literature linking PWA to exit from the workforce, little research has examined the extent to which age discrimination influences workers' abilities to meet the demands of their job or the process through which age discrimination influences outcomes. This study will address these gaps.

The first issue to consider in this area of research is the direct link between age discrimination and PWA. That is, does poor treatment influence workers' perceptions of 
their ability to perform their job duties? Furunes and Mykletun (2010) were able to establish a relation between age discrimination and PWA in their age discrimination scale development paper. However, this measure was designed to assess general age discrimination within a workplace, more in line with ageism climate (e.g., Elderly workers are not expected to take part in change processes and new working methods to the same degree as their younger peers), as opposed to an individual's personal experience of age discrimination. Moreover, this study used a measure of PWA that actually included a health item "What's your health compared with others of your own age?" which as noted in Study 1 confounds the PWA measure. Moreover, this study only included a cross-sectional data collection which may increase the common method bias associated with the findings from Furnes \& Mykletun (2010). Thus, there remains a need to establish the relation between individual perceptions of age discrimination and PWA using a time-lagged design to reduce common method variance (Podsakoff, MacKenzie, Lee, \& Podsakoff, 2003).

Second, in addition to linking age discrimination to PWA, research has not expanded this link to understand the extent to which age discrimination influences work and non-work outcomes indirectly though PWA. Given that PWA is negatively related to age (Bridger \& Bennett, 2011, Camerino et al., 2006) and is often studied in the context of the aging workforce; and given that justice perceptions are negatively linked to PWA (Bethge et al., 2012b), unfair treatment due to one's age, or age discrimination, is likely an important factor to consider for the development of poor PWA, but also for its subsequent outcomes. As a construct, PWA has only recently been integrated into the 
organizational psychology literature (Cadiz, Brady, Rineer, \& Truxillo, 2018), and additional research is needed to understand the role it plays as a mediating mechanism. As described in Study 1, I argue that PWA is likely to serve as a mediating mechanism between contextual workplace factors, including age discrimination, and work and nonwork outcomes. Despite the theoretical basis for these arguments, the extant research has not adequately tested this process.

Third, although PWA serving as a mediating mechanism is important in its own right, it is also fair to question the practical value of PWA when considering established constructs. For example, job self-efficacy and perceived fit share some conceptual overlap with PWA. Indeed, as identified in Study 1 and based on research by McGonagle and colleagues (2015), the relations between PWA and related constructs, such as perceived fit and job self-efficacy, tend to be moderate in size. Study 1 provided initial evidence that PWA explains unique variance in a range of outcomes when accounting for job self-efficacy and perceived fit, suggesting that PWA does provide value beyond previously established measures. However, that merely serves as an initial step toward more adequately establishing the value of PWA when other constructs have received decades or organizational research attention and may serve a similar function. Thus, this study seeks to provide additional evidence the PWA is a useful construct to consider as a mediating mechanism when simultaneously considering the role of perceived fit. If PWA remains an important mediating mechanism, that would provide additional support for the use of PWA within the organizational sciences as it serves a role unaddressed by other established constructs. 
Taken together, this study will draw on organizational justice theory and the JD$\mathrm{R}$ model to address the three gaps I have identified in the extant research, and in doing so, this study will contribute to the literature in a number of ways. First, this study will assess the influence of age discrimination on PWA, as well as the indirect link of age discrimination to retirement intentions, professional turnover intentions, and job performance. Second, this study will formally test PWA as a mediating mechanism between age discrimination and important outcomes, a process that is implied in the findings from Study 1, with other job demands and job resources, but not formally tested. Finally, this study will investigate whether PWA remains a mediating mechanism when simultaneously considering the established construct, perceived fit. Specifically, I will test both PWA and perceived fit as mediators between age discrimination and retirement intentions, professional turnover intentions, and job performance.

\section{Theoretical Background}

\section{Organizational Justice Framework}

Organizational justice theory posits that there are three primary components of organizational justice: distributive, procedural, and interactional (Gilliland, 1993; Greenberg, 1987, 1990). These aspects of justice form the basis of how individuals perceive fair treatment, and as such, violations of these aspects of justice are considered unfair treatment or a form of injustice. Within the justice framework, distributive justice refers to fairness in the allocation of resources (Gilliland, 1993; Greenberg, 1987, 1990), whereas procedural justice is considered fairness in the process used to allocate resources (Greenberg, 1993). In addition to distributive and procedural justice, which have received considerable research attention, interactional justice refers to interpersonal fairness in 
interactions, characterized by treating other with respect, and avoiding inappropriate comments or behaviors (e.g., discriminatory acts; Bies \& Moag, 1986). Meta-analytic evidence has shown that justice is related to a host of organizational outcomes including task performance, organizational citizenship behaviors, and counterproductive workplace behaviors (Colquitt et al., 2013). Given these links, perceptions of justice within organizations are clearly important to consider. Although justice is generally studied as perceptions of fair treatment in general, I argue that injustice as a function of group membership or other non-job related characteristics, including age discrimination, can be viewed as a form of injustice as well. That is, personal experiences of unfair treatment due to one's age violate the same expectations of fairness considered in organizational justice theory, and thus, age discrimination can be viewed as a specific form of injustice.

Although violation of any of the three forms of injustice may be associated with perceived age discrimination, the often subtle, but frequently occurring nature of discriminatory behaviors (Cortina, Kabat-Farr, Leskinen, Huerta, \& Magley, 2013) may be most strongly tied to interactional injustice. Given that people are able to pick up on non-verbal cues and more subtle forms of discrimination (e.g., Cortina et al., 2013; Gaertner \& Dovidio, 2005), it is likely that these perceptions of unfair treatment violate the justice rules posed by Bies and Moag (1986) for fair interpersonal treatment which are treatment with respect and propriety of communication. As an example, having one's opinion be valued less, or being treated poorly by coworkers or supervisors due to one's age, would clearly suggest a lack of respect for the individual. Indeed, these types of 
behaviors even make up existing age-discrimination scales (e.g., Marchiondo et al., 2015).

Job Demands-Resources Model

In addition to organizational justice theory, the job demands-resources (JD-R) model (Bakker \& Demerotui, 2007; Demerouti, Bakker, Nachreiner, \& Schaufeli, 2001) provides insight into how age discrimination is expected to be related to PWA and subsequent workplaces outcomes. Specifically, the JD-R model posits that job demands are aspects of the job that require sustained effort or skill and result in physiological or psychological costs (Bakker \& Demerouti, 2007). Researchers have also delineated job demands into two categories, challenge and hindrance demands. Hindrance demands are characterized as demands that have the potential to make work unnecessarily difficult (e.g., organizational politics, role conflict), whereas challenge demands can be stressful, but also facilitate goal attainment or personal growth (e.g., high workload, time pressure; Crawford LePine, \& Rich, 2010). Crawford and colleagues (2010) found that challenge demands can be motivating and are positively related to engagement, while also being positively associated with burnout. On the other hand, hindrance demands are negatively related to engagement and positively related to burnout. Applying the concepts of job demands and challenge versus hindrance demands to age discrimination, I argue that age discrimination can be defined as a job demand because dealing with age discrimination likely requires emotional and psychological effort to manage. Moreover, I argue that age discrimination can be viewed as a hindrance demand because it does not serve to foster personal growth or goal attainment, but would instead make an individual's work 
unnecessarily difficult and present unnecessary barriers to goal attainment. Given the classification of age discrimination as a hindrance demand, I draw on the JD-R model as well as organizational justice theory to develop hypotheses linking perceived age discrimination to PWA, perceived fit, and four job outcomes, retirement intentions, profession turnover intentions, organizational citizenship behaviors, and task performance (see Figure 3 for the conceptual model).

\section{Hypothesis Development}

Age discrimination refers to unfair treatment of an individual attributed to their age (Levy \& Banaji, 2002). This may include being passed over for a promotion, having one's ideas being valued less by others, or not being invited to social events by coworkers due to one's age (Marchiando et al., 2015). This type of unfair treatment is likely to have profound effects on an individual. Although relatively little research has focused on age discrimination compared to other forms of discrimination, age discrimination has been linked to intended retirement age (e.g., Bayl-Smith, \& Griffin, 2014; Snape \& Redman, 2003), health (e.g., mental health; Yuan, 2007) and job attitudes (e.g., job satisfaction; Marchiondo, Gonzales, \& Ran, 2015). Furthermore, research has shown older workers face lower ratings across many work domains including being viewed as less productive (e.g., Ng \& Feldman, 2012; Posthuma \& Campion, 2009) despite performing as well and sometimes even better than their younger counterparts according to some performance indicators (e.g., OCBs; Ng \& Feldman, 2008). The negative stereotypes against older workers have also been shown to increase rates of stereotype threat effects, where individuals fear conforming to age-based stereotypes (e.g., unable to learn new things) 
and end up performing congruent with those stereotypes not due to lower ability, but due to the stereotype itself (Lamont, Swift, \& Abrams, 2015).

In the context of an aging workforce, it is important to extend research on age discrimination to see what additional factors it negatively influences. Specifically, important age-related factors, such as one's ability to meet the demands of their job, or PWA, should be more thoroughly studied. Research has linked other forms of discrimination to reductions in constructs related to PWA, such as self-efficacy (e.g., racism, sexism; Schmitt, Branscombe, Postmes, \& Garcia, 2014), suggesting a negative relationship between age discrimination and PWA may occur in a similar manner. In addition, although not the primary focus of their study, one study found a negative relationship between age discrimination and ratings of personal resources (e.g., beliefs about their abilities) by older workers (Zaniboni, 2015). These findings suggests that age discrimination does influence individuals' perceptions of themselves, and thus, may influence PWA as well because PWA reflects perceptions of their capacity to do their jobs. Specifically, when individuals face high levels of age discrimination, they may need to dedicate more of their resources (e.g., emotional, mental) to cope with the unfair treatment they experience, thereby, leaving fewer available resources to meet the demands of their job. Fewer available resources to meet the demands of one's job are then expected to result in lower PWA. That is, if a worker faces age discrimination they are likely to perceive the work context as being unnecessarily difficult or having unnecessary roadblocks to the completion of their work. Thus, drawing on the JD-R model, when faced with high levels of age discrimination - a hindrance demand - I posit 
that workers will need to expend more resources to meet the demands of their job, and thus report lower levels of PWA.

Hypothesis 1: Age discrimination will be negatively related to PWA. Age Discrimination and Retirement Intentions

Given that intentions are an antecedent to actual behavior (Ajzen, 1985) and that this link has been supported for retirement intentions and exit from the workforce (Feldman, 1994), attitudes towards exiting the workforce are particularly important for organizations to consider. In line with research on "push" and "pull" factors influencing retirement (Shultz, Morton, \& Weckerle, 1998), feeling ostracized from one's workplace may serve as a "push" factor that encourages an individual to exit, or at least consider exiting the workforce. This also aligns with research on organizational justice, which has found negative relationships between justice and withdrawal and negative reactions, and a positive relationship between justice and job performance (Colquitt et al., 2001; Colquitt et al., 2013).

Age Discrimination and Profession Turnover Intentions

Beyond exiting from the workforce entirely, feeling mistreated in the workplace due to one's age is also likely to have other negative consequences. Although retirement may be impossible for financial or other personal reasons, a more practical step may be to consider alternative occupations for employment. That is, if an individual no longer feels accepted or capable of working in a given role, they may turn to a role in which they feel more accepted and capable. An example of how this may occur is moving from a traditionally youth-oriented role, such as working in the fashion industry, and turning to a 
role that better fits the stereotypes of one's age, such as a banker. Similar processes have been observed among other demographic groups; for example, women who are exposed to the stereotype that women do not perform as well in math report a lower preference for majoring in math (Davies, Spencer, Quinn, \& Gerhardstein, 2002).

Age Discrimination and Job Performance

In addition to the proposed links between age discrimination and retirement and profession turnover intentions, individuals that face age discrimination may actually perform worse at work. Importantly, job performance can broadly be categorized as task performance, which includes in-role behaviors, and organizational citizenship behaviors (OCBs; Williamson \& Anderson, 1991), which involve behaviors that contribute to performance of the organization, but may not be formally defined in the employees' responsibilities (e.g., helping behaviors; Borman \& Motowidlo, 1997; Campbell \& Wiernik, 2015; Motowidlo \& Van Scotter, 1994).

Organizational citizenship behaviors. Individuals facing high levels of age discrimination may be less likely to engage in OCBs because they are being mistreated by those around them (e.g., their coworkers, supervisor) or by the organization in general (e.g., organizational policies perceived as discriminatory). Within the JD-R framework, age discrimination, which can be considered a hindrance demand, would require more resources to deal with, and as such, workers may focus more narrowly on the most critical aspects of performance and eschew informal contributions to their workplace (e.g., helping behaviors, informal training). Indeed, Colquitt and colleagues (2001) found that justice was associated with greater withdrawal behaviors. Given that OCBs are 
generally social in nature (e.g., covering a shift for a sick colleague), experiencing unfair treatment would substantially demotivate individuals to engage in OCBs which have a distinct social component to them. Thus, I argue that when an individual perceives age discrimination they will withdrawal from the social context of their workplace and engage in fewer OCBs.

Age Discrimination and Task Performance

In addition to the expected negative effect of age discrimination on OCBs, I argue that age discrimination will be negatively related to task performance. Older workers facing age discrimination may experience stereotype threat reductions in performance (Lamont, Swift, \& Abrams, 2015). However, it is perhaps more likely, that when exposed to age discrimination individuals become disengaged, unmotivated, and simply upset at their workplace or those they are working with. Indeed, research has shown that when experiencing unfair treatment, individuals may become less motivated (Zapata-Phelan, Colquitt, Scott, \& Livingston, 2009), perform worse in terms of their task performance, and even engage in counterproductive workplace behaviors (Colquitt et al., 2013). Further, within the JD-R framework, experiencing high levels of hindrance demands are expected to be negatively related to performance, of which, task performance is a key indicator. Taken together, based on organizational justice theory, the JD-R model, and extant research on age discrimination, it is hypothesized that age discrimination will be related to higher levels of retirement intentions and profession turnover intentions, and lower levels of OCBs and task performance. 
Hypothesis 2: Age discrimination will be positively related (a) retirement intentions and (b) profession turnover intentions and negatively related to $(c)$ organizational citizenship behaviors and (d) task performance.

Work Ability and Perceived Fit as Mediators

Although the direct link from age discrimination to job outcomes is important, it is also important to identify potential mechanisms that may influence these relationships. As demonstrated in Study 1, PWA is an antecedent of a range of important job outcomes including job attitudes, exit behaviors, and performance. As such, I anticipate it will be related to such outcomes in the present study. However, in the face of discrimination and unfair treatment, an individual's WA, particularly perceptions of their WA, may be harmed. That is, an individual may initially become frustrated or hurt, but over time, the experience of mistreatment may have more profound negative effects, such as reducing one's beliefs about their own abilities (i.e., people may internalize this discrimination) which would be reflected by lower levels of PWA. Indeed, discrimination has not only been linked to lower levels of self-efficacy (Schmitt et al., 2014), but also to negative health outcomes (Pascoe \& Richman, 2009). Given the strong ties between PWA and health (McGonagle et al., 2015, Nevampera et al., 2016) the negative effects of age discrimination on PWA are likely to lead to poorer workplace outcomes. In line with organizational justice theory and the literature suggesting there may be a link between age discrimination and WA, as well as evidence provided in Study 1 showing that PWA is related to important job outcomes, the following hypothesis was developed. 
Hypothesis 3: PWA will be negatively related to a) retirement intentions and b) profession turnover intentions and positively related to (c) OCBs and (d) task performance.

Hypothesis 4: The relationship between age discrimination and (a) retirement intentions, (b) profession turnover intentions, (c) OCBs, and (d) task performance will be mediated by $P W A$.

Although PWA is the primary variable of interest in this study, the conceptually related construct, perceived fit, could also serve this mediating role between age discrimination and job outcomes. Perceived fit refers to a match between one's desires, needs, and preferences, or one's knowledge, skills, and abilities, and the environment (Edwards, 1991) and has been linked to important job outcomes (e.g., job satisfaction, organizational commitment; Kristof-Brown, Zimmerman, \& Johnson, 2005). If individuals experience age discrimination they may feel that they no longer fit as well in their current role, and thus, be more likely to leave via retirement or turnover, or turnover. Indeed, some research has shown that certain forms of discrimination are related to lower levels of person-organization fit (e.g., LGBTQ; Velez \& Moradi, 2012) and lower perceptions of perceived fit, have been linked to organizational commitment and satisfaction (Kristoff-Brown et al., 2005).

In addition to identifying the role WA plays between age discrimination and job outcomes, it is critically important that research also provide evidence that WA predicts these important outcomes when considering related and established constructs (Cadiz et al., 2018). Due to the conceptual overlap between WA and perceived fit, it could be that 
when considered together the two constructs overlap in such a way that they are essentially redundant with one another and provide minimal unique value. However, despite some conceptual overlap, as detailed in Study 1, WA and perceived fit have several distinct characteristics. First, in terms of measurement, perceived fit takes into consideration an individual's values and wants, rather than strictly their ability (Cable \& DeRue, 2002). That is, an individual may have a deep desire to work in a given role, such as continuing to work as a construction worker later in life, indicating high perceived fit, but actually be struggling profoundly to meet the demands of that job (e.g., due to healthrelated declines or problems), and thus have low WA. In order to provide greater support for using PWA in the organizational sciences, it is important to demonstrate that PWA remains an important construct (i.e., mediating mechanism) when considering perceived fit - as proposed in the following hypotheses. Given the distinctions between PWA and perceived fit as well as evidence that both are expected to serve as mediators between age discrimination and outcomes of interests, I hypothesize that both PWA and perceived fit will serve as mediating mechanisms between age discrimination and the outcomes of interests.

Hypothesis 5: The relationship between age discrimination and (a) retirement intentions, (b) profession turnover intentions, (c) OCBs, and (d) task performance will be mediated by perceived fit.

Hypothesis 6: Both PWA and perceived fit, will serve as mediators between age discrimination and (a) retirement intentions, (b) professional turnover, (c) OCBs, and (d) task performance. 


\section{Method}

Sample

The study sample consisted of nurses working in the Pacific Northwest. The nurses were recruited through the Oregon Nursing Association and received \$20-30 in the form of Amazon gift cards per survey completed. Data were collected at three time points, including baseline, Time 2 (3 months later), and Time 3 (6 months after baseline). At Time 1, 121 participants completed the survey, at Time 2, 109 completed the survey, and at Time 3, 98 participants completed the survey. In total, 82 participants completed each wave of data (see Table 10 for sample descriptive statistics of the sample at baseline). Perceived age discrimination was collected at baseline, the mediators, PWA and perceived fit were collected at Time 2 (3 months), and the outcomes - retirement intentions, profession turnover intentions, organizational citizenship behaviors, and task performance - were collected at Time 3 (6 months). Note, in analyses for retirement intentions, the SEM model was estimated using only workers age 50 or older, which includes $n=50$ workers.

Measures

Perceived age discrimination was assessed using a 9-item scale from Marchiando and colleagues (Marchiondo, Gonzales, \& Ran, 2015). A sample item is "I have been treated with less respect because of my age." Items were scored on a 7 point scale from (1 $=$ strongly disagree to $7=$ strongly agree) with higher scores indicating more perceived age discrimination. As evidenced by the sample item in this scale, many items included in the age discrimination scale represent specific facets of the organizational justice 
framework (e.g., interpersonal justice for the sample item). This scale demonstrated excellent internal consistency $(\alpha=.97)$.

Perceived WA was assessed with four items drawn from Morschhäuser and Sochert (2007) and adapted by McGonagle and colleagues $(2014,2015)$. Three items are scored on a five-point scale assessing one's ability compared to the 1) mental, 2) physical, and 3) interpersonal demands of the job. A fourth item assessing one's "current work ability compared to your lifetime best" used an 11-point scale from $(0=$ completely unable to work to $10=$ work ability at its lifetime best). Scores on each item were summed together with higher scores indicating greater PWA. This scale has demonstrated acceptable internal consistency $(\alpha=.76)$.

Perceived fit was assessed with 9 items from Cable and DeRue, 2002. The scale assesses three facets of fit, person-organization, needs-supplies, and demands-abilities fit. A sample item is "My personal abilities and education provide a good match with the demands that my job places on me.” Responses were scored on a 7-point scale from $(1=$ strongly disagree to $7=$ strongly agree). Higher scores indicated greater perceptions of perceived fit. The perceived fit scale demonstrated excellent internal consistency $(\alpha=$ .91) when treated as a unidimensional scale.

Retirement intentions were assessed with three items adapted from Hom and colleagues (1984). A sample item is "I am planning on retiring in the near future." Responses are scored on a ( $1=$ strongly disagree to $5=$ strongly agree $)$ scale with higher scores indicating greater levels of retirement intentions. In this sample, the scale demonstrated good internal consistency $(\alpha=.83)$. Because retirement is likely not 
possible for younger workers, analyses reporting retirement intentions as an outcome were limited to participants that were at least 50 years old.

Professional turnover intentions were also assessed with three items adapted from Hom and colleagues (1984). A sample item is "I often think about quitting this profession." Responses are scored on a ( $1=$ strongly disagree to $5=$ strongly agree $)$ scale with higher scores indicating greater profession turnover intentions. The profession turnover intentions scale demonstrated acceptable internal consistency $(\alpha=.73)$.

Job performance. Job performance was assessed in terms of organizational citizenship behaviors (OCBs), which were self-rated and assessed with 7 items from Williams and Anderson (1991). Responses were scored from 1 to 5 (strongly disagreestrongly agree), with higher scores indicating greater performance. A sample item is "help others who have heavy workloads." Note, this scale includes 11 total items and two subscales. One subscale includes behaviors directed at other individuals (OCBIs), and the other subscale includes behaviors directed toward the organization in general (OCBOs). In the present study, the OCBO items did not correlate well with each other, resulting in a poor alpha $(\alpha=.51)$. Removing the most problematic item led to only marginal increases in internal consistency $(\alpha=.52)$. When considering all OCB items together, the scale demonstrated acceptable internal consistency $(\alpha=.75)$. However, when including only the OCBO items, a great level of internal consistency was found $(\alpha=.80)$. Overall, this suggests that within this specific context and sample, the OCBO items do not necessarily address a unified underlying concept. Thus, based on the lack of internal consistency among the items, I decided to focus on the OCBI subscale as an indicator of 
organizational citizenship behaviors for the present study. Task performance was assessed with three items from Griffin, Neal, and Parker (2007). A sample item is the extent to which you have "completed your core tasks well using the standard procedures." The scale was scored on a 1-7 (strongly disagree-strongly agree) scale, which demonstrated excellent internal consistency $(\alpha=.93)$.

Analytic Strategy

Analyses were conducted in MPus version 8.1 using structural equation modeling (SEM) with scale scores included as observed variables. Observed scale scores were utilized as opposed to observed items loading onto latent factors due to the limitation of statistical power. Analyses were conducted using Full Information Maximum Likelihood (FIML) to account for missing data. Indirect effects were assessed with bias corrected bootstrapping, using 10,000 iterations and interpretation of the indirect effects were based on the standardized $95 \%$ confidence intervals of the bootstrapping analysis. Confidence intervals that did not include zero were considered an indication of a significant indirect effect.

Three SEM models were used to evaluate Hypotheses 1-6. Model 1 included age discrimination, PWA, and the outcomes of interest in order to evaluate Hypotheses 1-4. Specifically, in Model 1 PWA was regressed on age discrimination (Hypothesis 1), each outcome was regressed on age discrimination (Hypothesis 2a-d), and the outcomes of interest were regressed on PWA (Hypothesis 3a-d). In addition, the indirect effects of age discrimination to the three outcomes through PWA were specified (Hypothesis 4a-d). The second SEM model included the outcomes of interest being regressed on age 
discrimination, perceived fit being regressed on age discrimination, and the outcomes on interest being regressed on perceived fit. Further, the indirect effect of age discrimination on the outcomes of interest through perceived fit were specified (Hypothesis 5a-d). To evaluate Hypothesis 6a-d, which incorporates both PWA and perceived fit, an SEM model specifying age discrimination as the $\mathrm{x}$ variable, PWA and perceived fit as the mediators, and retirement intentions, profession turnover intentions, task performance, and OCBIs as the outcomes was specified.

\section{Results}

As noted in the Method section, each scale demonstrated acceptable internal consistency, see Table 10 for the intercorrelations among study variables and descriptive statistics for each scale. As seen in the correlation matrix, age discrimination was negatively correlated with both PWA and perceived fit, and PWA was positively correlated with task performance. Notably, correlations were not significant unless they were approximately $r=.20$ in magnitude.

Hypothesis 1 stated that age discrimination would be negatively related to PWA. In the first SEM model, age discrimination was indeed negatively related to PWA, $\beta=$ $.308, p=.003$. Thus, Hypothesis 1 was supported, indicating higher levels of age discrimination are associated with lower levels of PWA.

Hypothesis 2 stated that age discrimination would be negatively related to a) retirement intentions and b) profession turnover intentions, but positively related to c) OCBs and d) task performance. The standardized model results indicated there was a direct effect of age discrimination on profession turnover intentions, $\beta=.277, p=.008$, 
but not on retirement intentions $\beta=.009, p=.948$, organizational citizenship behaviors, $\beta$ $=-.012, p=.882$, and task performance, $\beta=-.145, p=.133$. Thus, I found weak support for Hypothesis 2. Specifically, only Hypothesis $2 b$ was supported.

Hypothesis 3 was that PWA would be negatively related to retirement intentions and profession turnover intensions, but positively related to OCBs and task performance. Based on the standardized model results, PWA was positively related to task performance, $\beta=.236, p=.023$ and negatively related to retirement intentions $\beta=-.398, p$ $=.008$. However, the relations between PWA and profession turnover intentions, $\beta=$ $.012, p=.905$ and PWA and OCBs were not significant $\beta=.191, p=.145$. Thus, Hypothesis 3 received mixed support.

Hypothesis 4 stated that age discrimination would be indirectly related to the outcomes of interest through PWA, with PWA serving as a mediating mechanism. The indirect effect of age discrimination through PWA was significant for retirement intentions and task performance, but not for profession turnover intentions or OCBs. Specifically, the $95 \%$ confidence intervals for the indirect effects on retirement intentions [95\% CI, LL: .031, UL: .347] and task performance [95\% CI, LL: -.189, UL: -.007] did not include zero. On the other hand, the $95 \%$ confidence intervals for profession turnover intentions [95\% CI, LL: -.074, UL: .059] and OCBs [95\% CI, LL: -.186, UL: .013] did include zero, and thus, were not significant. Taken together, similar to Hypothesis 3, I found mixed support for Hypothesis 4.

Hypothesis 5 was that age discrimination would be indirectly related to outcomes of interest through perceived fit. In this case, the indirect effect of age discrimination on 
profession turnover intentions was significant, [95\% CI, LL: .003, UL: .184], however, the indirect effects on retirement intentions [95\% CI, LL:-.021, UL: .273], OCBs [95\% CI, LL: -.128, UL: .008], and task performance [95\% CI LL: -.095, UL: .021] were not significant. Overall, this finding provides minimal support for Hypothesis 5.

Hypothesis 6 was that when accounting for perceived fit and PWA together, each would still serve as a mediating mechanism between age discrimination and outcomes of interest. Overall, when considering both mediators simultaneously, the indirect effect of age discrimination through PWA was only significant for task performance, [95\% CI, LL: -.190, UL: -.012] and retirement intentions [95\% CI, LL: .024, UL: .307]. For perceived fit, the indirect effect of age discrimination was only significant for profession turnover intentions, [95\% CI, LL: 004, .204]. For the remaining indirect effects, the 95\% confidence intervals included zero, and thus, were not significant. Based on three of the possible eight indirect effects being significant, Hypothesis 6 received mixed support (see Figure 4 for the full path model with direct and indirect effects).

Summary of Results

Taken together, Hypothesis 1 which posited age discrimination would be negatively related to PWA was supported. However, for the remaining hypotheses, each received only mixed support. Hypothesis 2 which argued age discrimination would be related to the outcomes of interest was only supported for profession turnover intentions. Hypothesis 3, that PWA would be related to retirement intentions, profession turnover intentions, OCBs, and task performance, was supported in regard to task performance and retirement intentions, but not profession turnover intentions or OCBs. Hypothesis 4 
argued that age discrimination would be related to the outcomes of interest through PWA. Again, this received mixed support. Specifically, the mediated relation was significant for task performance and retirement intentions. Hypothesis 5 stated the perceived fit would mediate the relations between age discrimination and the outcomes of interest. However, the mediating effect through perceived fit was only supported for profession turnover intentions. Finally, Hypothesis 6 posited that when considering PWA and perceived fit, each would serve as mediators between age discrimination and outcomes of interest. However, this process was supported for task performance and retirement intentions through PWA and only for profession turnover intentions through perceived fit. Supplemental Analyses

Based on the lack of consistent support for Hypotheses 4-6, of PWA and perceived fit serving as mediators between age discrimination and outcomes of interest, I sought to further explore if the mediating process from age discrimination to outcomes of interests through PWA and perceived fit would be found with other outcomes. Based on organizational justice theory, perceptions of injustice (i.e., age discrimination) can have negative consequences for a range of outcomes (e.g., social exchange quality, job performance; Colquitt et al., 2013; job satisfaction, organizational commitment, turnover intentions; Cohen-Charash \& Spector, 2001). Similarly, based on the JD-R model, the negative outcomes of hindrance demands can be relatively broad and include engagement, burnout, worse job attitudes, and reduced performance (Bakker \& Demerouti, 2007; Demerouti et al., 2001). As such, I sought to evaluate a wider range of potential outcomes associated with age discrimination through PWA and perceived Fit. 
Engagement and wellbeing. First, within the JD-R model, engagement and wellbeing are often viewed as more proximal outcomes of job demands than job attitudes and performance outcomes. Thus, concerning age discrimination, which I argue is a specific hindrance demand; I first investigated the extent to which age discrimination was negatively related to engagement and wellbeing through PWA and perceived fit. Based on the JD-R model, age discrimination was expected to be negatively related to engagement and related to lower levels of wellbeing. Based on the available data, wellbeing was operationalized as burnout, stress, and presenteeism (i.e., number of days sick at work) for these subsequent analyses. In the follow up analyses, both PWA [95\% CI, LL: -.201, UL: -.013] and perceived fit [95\% CI, LL: -.146, UL: -.004] served as mediators of the relation between age discrimination and engagement. However, only PWA was a significant mediator for stress [95\% CI, LL: .017, .211] and presenteeism [95\% CI, LL: .023, UL: .265], whereas only perceived fit was a significant mediator for burnout [95\% CI, LL: .009, UL: .186].

Attitudinal variables. Second, within the context of the JD-R model, job demands are expected to be related to lower levels of positive attitudes (e.g., job and life satisfaction) and are commonly investigated as outcomes in the JD-R model. For these follow up analyses, I investigated job satisfaction, affective organizational commitment, and life satisfaction as outcomes. When accounting for both mediating variables, PWA did not mediate the relations between age discrimination and job satisfaction [95\% CI: LL:-.137, UL: .033] or organizational commitment [95\% CI: LL: -.012, UL: .154], but did mediate the relation between age discrimination and life satisfaction [95\% CI: -.198, 
UL: -.010]. On the other hand, perceived fit did mediate the relations between age discrimination and job satisfaction [95\% CI, LL:-.167, UL: -.003] and organizational commitment [95\% CI: LL: -.257, UL: -.005], but not life satisfaction [95\% CI: LL: -.107, UL: .031].

Age specific variables. Last, age discrimination and PWA are commonly studied within the context of an aging workforce. In addition, researchers have imbedded perceived fit into the context of an aging workforce (Perry, Dokko, \& Golom, 2012; Zacher, Feldman, \& Schulz, 2014). Thus, age discrimination, perceived fit, and PWA are believed to be important for working successfully later in life. As such, I conducted follow-up analyses with age specific variables as the outcomes of interest. Specifically, I included generativity motives, growth motives, and morale age for these analyses. PWA served as a mediator for growth motives [95\% CI, LL: -.220, UL: -.010] whereas perceived fit served as a mediator for morale age [95\% CI, LL: -.224, UL: -.006].

Age Discrimination across the Lifespan. Nursing is a unique context in which both older and younger nurses experience discrimination due to their age. That is, older nurses have been noted as "eating their young" whereas older nurses themselves are also discriminated against in the late stages of their career. Thus, age discrimination may yield differential effects across the lifespan. As evidence to support this narrative, I inspected the scatterplot diagram between age and age discrimination. The plot visually suggested the potential for a curvilinear relation between age and age discrimination. Thus, I computed a quadratic age variable, an age by age interaction (centered prior to computing the interaction). Through regression analysis, I input age discrimination as the dependent 
variable and found that both the centered age variable, $\beta=.240, p=.019$ as well as the quadratic age term $\beta=.303, p=.003 \Delta R^{2}=.07$ explained significant amounts of variance in age discrimination. Further, the quadratic age term explain a relatively robust $7 \%$ of the variance in age discrimination after accounting for the linear age term.

\section{Discussion}

This study makes a number of contributions to the literature and points to several important directions for future research. First, although the findings were mixed, this study does provide some evidence that PWA can serve as a mediator between age discrimination and important outcomes (e.g., task performance, retirement intentions). Second, this study shed light on the substantial negative consequences of age discrimination, a particularly important topic for the aging workforce. Third, this study expanded upon the theoretical mediators of age discrimination to outcomes, which have previously focused on perceptions of justice, to show that the negative effects of age discrimination can occur through other pathways as well.

As the workforce ages and becomes more age-diverse, the treatment of workers based on their age (age discrimination) and perceptions of older workers' ability to continue working (perceived work ability) will become increasingly important. Indeed, in this study, age discrimination was negatively related to PWA and perceived fit and positively related to profession turnover intentions. In addition, PWA was positively related to task performance, a key outcome for organizations and retirement intentions. There were also multiple indirect effects from age discrimination through PWA, including the indirect negative effect on task performance and the positive indirect effect 
on retirement intentions. Perceived fit played a mediating role between age discrimination and profession turnover intentions, but not the remaining outcomes of interest. Although the link between PWA and OCBs as well as the indirect effect from age discrimination to OCBs through PWA was not supported, in retrospect this may be somewhat less surprising. That is, PWA reflects an individual's ability to perform their job duties, which are directly applicable to one's in-role or task performance. However, PWA does not address the social context in which OCBs occur, and thus, may be unrelated or less strongly related to OCBs. Rather, factors such as social support and a positive workplace climate are likely to be more important for OCBs, whereas for in-role behaviors, PWA may play a more influential role.

Supplementary analyses revealed additional indirect effects from age discrimination through PWA and perceived fit, which were quite nuanced. For instance, among attitudinal variables, age discrimination was indirectly related to life satisfaction through PWA. On the other hand, for job satisfaction and organization commitment, two important job attitudes, perceived fit remained a significant mediator. This suggests PWA may be more important for non-work outcomes, whereas perceived fit may be particularly important for affective job attitudes. Regarding engagement and wellbeing, age discrimination was indirectly related to engagement through both PWA and perceived fit. PWA also played a mediating role for perceived stress and presenteeism, whereas perceived fit played a mediating role between age discrimination and emotional exhaustion. Finally, regarding age-specific variables, PWA played a mediating role between age discrimination and growth motives, whereas perceived fit played a 
mediating role between age discrimination and morale age (i.e. attitudes toward aging). Taken together, although the specific instances of mediation were inconsistently supported, the overarching concept that age discrimination may influence work and nonwork outcomes through PWA received moderate support.

Theoretical Contributions

Although the findings of WA as a mediator were mixed, the results of this study still support a fundamental and underlying point of extending upon organizational justice theory to incorporate specific types of justice. That is, organizational justice theory considers justice in terms of distributive, procedural, and interactional justice. However, I argue that organizational justice theory can also take the form of justice based on a specific personal characteristic as an application of interactional injustice. Specifically, age discrimination was posited as a specific form of injustice, which should lead to a range of important outcomes, and be negatively related to WA and perceived Fit. Indeed, as would be expected from other aspects of justice (e.g., distributive, procedural justice), age discrimination was negatively related to PWA, perceived fit, and profession turnover intentions. In addition, the indirect effects of age discrimination through either PWA or perceived fit were supported for job satisfaction, organizational commitment, life satisfaction, engagement, stress, burnout, presenteeism, growth motives, and morale age. Thus, this study offers initial support for integrating age discrimination research within the organizational justice theory framework.

Practical Contributions 
As the workforce ages, the ability to work in age-diverse settings will become increasingly important, and the impact of the aging workforce will be particularly hard hitting in specific fields, of which nursing is one. Thus, the present study yields important insights into the deleterious effects of age discrimination and points to why it is important for organizations to continue working to stamp out and mitigate it. Specifically, this research highlights that the negative effects of age discrimination within the field of nursing are particularly problematic. That is, age discrimination was related to greater intentions to leave the nursing profession, PWA, and perceived fit. Given that the nursing profession is facing an imminent shortage of workers, that nurses are on average somewhat older compared to workers in other professions, and that younger nurses also face age discrimination in nursing, this overarching finding is particularly worrisome.

Second, although age discrimination may be thought to have negative outcomes through justice perceptions directly, this study highlights that age discrimination may also manifest in reducing one's PWA and even their perceived fit with a given job. As such, organizations with high levels of age discrimination may seek to target age discrimination, but also to target methods for enhancing PWA and perceived fit as potential avenues for reducing the desire to leave the nursing profession, reducing burnout, and keeping nurses engaged and seeking growth opportunities within the nursing field.

Limitations and Future Directions

Although this study makes important contributions to the existing literature, it is not without limitations. First, the sample size in this study is relatively small, with only 
121 participants at Time 1 and 82 participants completing all three waves of data collection. Moreover, for the retirement intentions analyses, the analyses were further restricted to the $n=50$ employees that were at least 50 years old. The additional restriction is important as retirement intentions below age 50 seem unreasonable to consider for the vast majority of workers. However, as argued by Koopman and colleagues (2015), the use of bias corrected bootstrap analyses on such small sample may result in a relative overconfidence of the indirect effects (Koopman, Howe, Hollenbeck, \& Sin, 2015). Thus, findings from this study should be interpreted with some degree of caution. That said, the small sample and the lower statistical power resulting from it suggests that significant results are less likely to be detected. Thus, the results that were supported with the present sample are likely to also be supported with a larger sample size, and those hypotheses that were not supported should be tested in larger samples with greater statistical power. Although the sample size is a limitation, the use of FIML helps to reduce those effects and all analyses were ultimately assessed with the full 121 observations of data. In addition, although the sample size is admittedly smaller than ideal, the study was conducted in a profession of keen importance to society in general and to the aging workforce in particular: the nursing population is rapidly aging, and it is thus an occupation that is important to consider in terms of the effects of age discrimination on work ability.

Second, all of the data from this study are self-report. Thus, the performance measures as well as other indicators may be subject to self-rating biases. However, in order to compensate for this potential limitation, the antecedent, mediator, and outcome 
variables are separated by three data collection time points, and each variable is measured at the time point in which it was theoretically important to assess it. Thus, although the self-report dataset is certainly a limitation to acknowledge, self-report surveys are relatively standard practice in the organizational psychology literature and the nurses individual perception were largely the constructs of interest for the research questions addressed in this study (e.g., personal ratings of age discrimination, work ability, perceived fit). Future research should examine these issues using data collected from other sources (e.g., supervisor ratings of performance; see Study 3).

Third, nursing is a unique occupation in which both older and younger workers may face high levels of age discrimination. Indeed, based on scatter plots of age and age discrimination, it appears there may be a small group of nurses within this sample that are younger and facing high levels of age discrimination. Thus, although the overarching arguments center at times around challenges of the aging workforce, it is perhaps more appropriate to consider these challenges as those of an age-diverse workforce. Although this is potentially a concern, analysis run in which workers under age 30 were excluded yielded similar patterns of results compared to using the full sample. Future research should address these potentially different experiences with age discrimination and the extent to which age discrimination may lead to different outcomes dependent on age. Taken together, the findings from this study suggest that future research should continue to investigate the effects of age discrimination within nursing and healthcare settings for their effects on work ability, performance, and retirement decisions. 
Finally, in certain occupations, workers are treated and stereotyped as fitting differently across the lifespan, and nursing represents a unique case of this potential issue. In the primary analyses of this study, age discrimination was treated as having linear effects on the mediators and outcomes of interest. However, I found evidence that age discrimination is most prevalent at both the younger and older ends of the age spectrum. Thus, age discrimination may have differential effects on workers at different ages. For example, future research should explore if age discrimination against younger workers is the primary driver of the negative relation between age discrimination and perceived fit as well as the indirect effect from age discrimination to profession turnover intentions. Specifically, younger nurses may enter the field, experience age discrimination, and immediately question whether or not they want to remain in nursing long term. On the other hand, older nurses experiencing age discrimination may try to engage in adaptive behaviors, switch departments, or otherwise adjust their work environment, but not view a career change as a viable option. Future research should attempt to disentangle these potentially differential effects. Conclusion

This study provided insight into the existing literatures of age discrimination and PWA, two increasingly important constructs to consider as industrialized workforces age. Although hypotheses across various outcomes of interest were not always supported, age discrimination was linked directly to PWA, perceived fit, and profession turnover intentions. Further, age discrimination was shown to influence a wide range of work and non-work outcomes (e.g., life satisfaction, growth motives, engagement), indirectly 
though PWA or perceived fit. Finally, the finding that in many cases only PWA or perceived fit served as a mediating mechanism suggests that the two constructs are in fact unique, and that considering PWA or perceived fit together does yield important information that would be missed by focusing on either the more established construct, perceived fit, or the more recently studied construct in the organizational sciences, PWA. 
Figure 3 Study 2 Conceptual Model

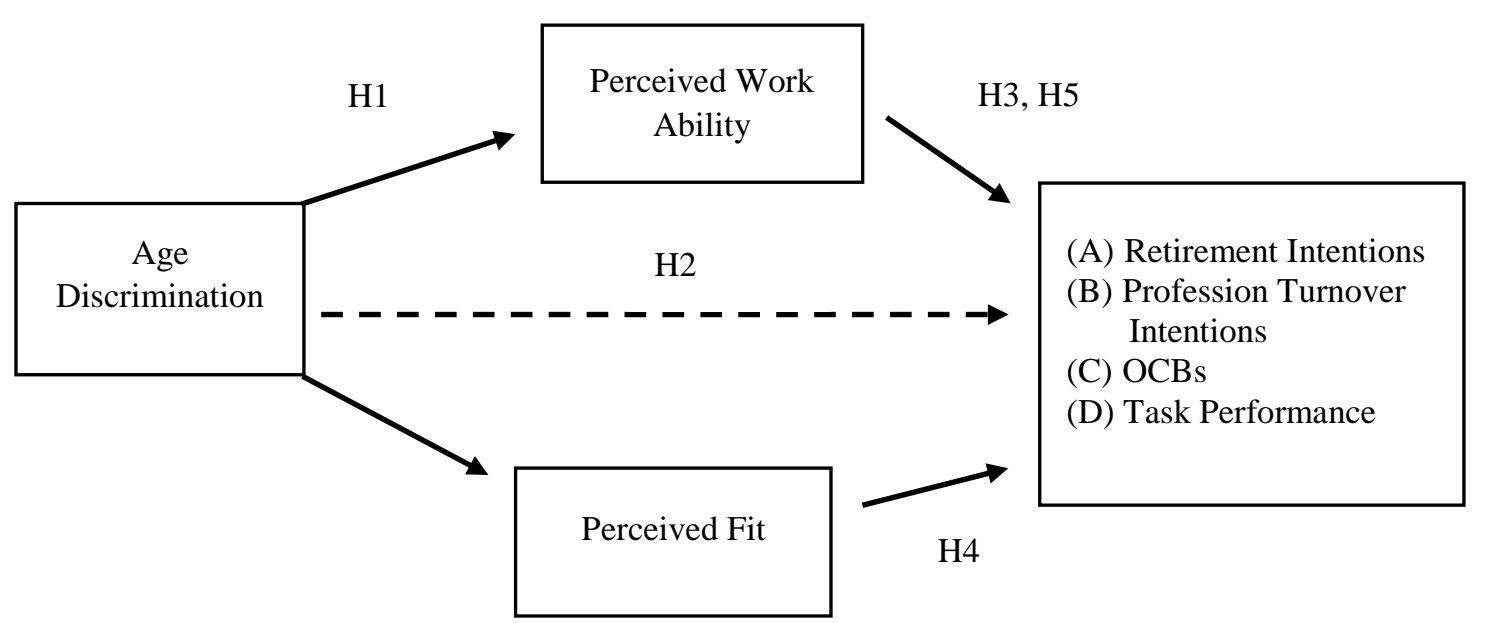

Figure 3. Theoretical model of the proposed hypotheses between age discrimination and work outcomes via two mediating variables, work ability and perceived fit. 
Table 9. Study 2 Sample Descriptive Statistics

\begin{tabular}{ll}
\hline Sample characteristic & $M(\mathrm{SD})$ or \% of the sample \\
\hline Age & $49.15(12.70)$ \\
Ethnicity (Percent White) & $115(95.04 \%)$ \\
Organizational Tenure (Years) & $11.87(11.91)$ \\
Hours Worked & $33.6(8.40)$ \\
Education & \\
$\quad$ Some college/technical school & $7(5.8 \%)$ \\
$\quad$ College 4 years or more & $70(57.9 \%)$ \\
$\quad$ Graduate Degree & $27(22.3 \%)$ \\
$\quad$ Two-year RN degree & $17(14 \%)$ \\
Job Tenure (Years) & $7.56(8.32)$ \\
Supervisory Role (yes) & $41(33.90 \%)$ \\
\hline Note. Total sample size was 121 at Time 1.
\end{tabular}


Figure 4 Study 2 Results

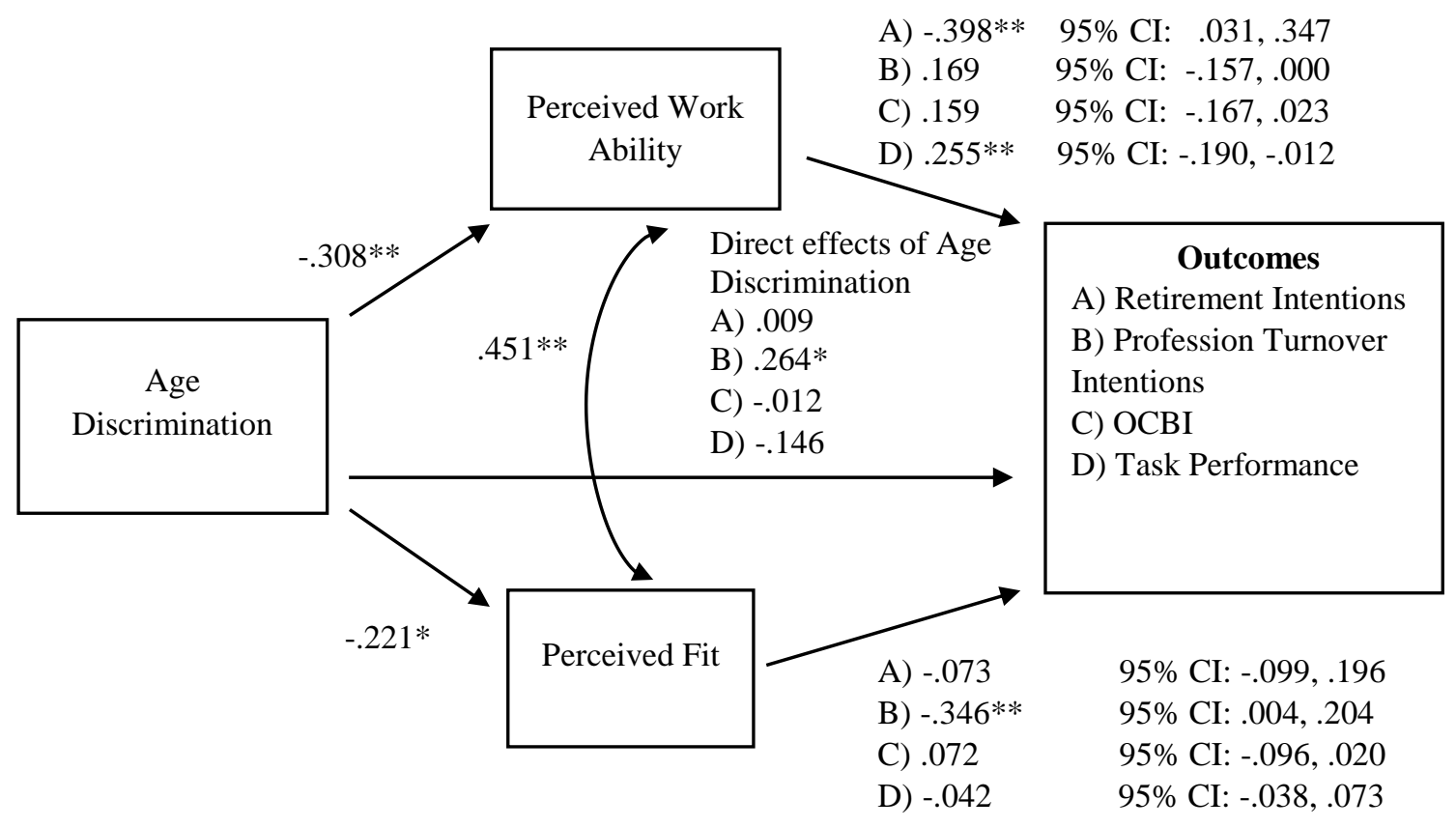

Note. A) Indicates direct effect of the mediating variable on retirement intentions, follow by the $95 \%$ CI for the indirect effect of age discrimination on retirement intentions through each respective mediator. B) Indicates direct effect of the mediating variable on profession turnover intentions, follow by the $95 \% \mathrm{CI}$ for the indirect effect of age discrimination on profession turnover intentions through each respective mediator. C) Indicates direct effect of the mediating variable on organizational citizenship behaviors, follow by the 95\% CI for the indirect effect of age discrimination on organizational citizenship behaviors through each respective mediator. D) Indicates direct effect of the mediating variable on organizational citizenship behaviors, follow by the $95 \% \mathrm{CI}$ for the indirect effect of age discrimination on organizational citizenship behaviors through each respective mediator. 
Table 10

Means, Standard Deviations, and Zero -Oder Correlations among Study 2 Variables.

\begin{tabular}{|c|c|c|c|c|c|c|c|c|}
\hline & & $\mathrm{M}(\mathrm{SD})$ & 1 & 2 & 3 & 4 & 5 & 6 \\
\hline 1 & T1 Age Discrimination & $2.47(1.51)$ & & & & & & \\
\hline 2 & T2 Perceived WA & $20.17(3.0)$ & $-.30^{* *}$ & & & & & \\
\hline 3 & T2 Perceived Fit & $5.04(1.07)$ & $-.20^{*}$ & $.48^{* *}$ & & & & \\
\hline 4 & T3 OCBI & $4.39(.55)$ & -.13 & .20 & .14 & & & \\
\hline 5 & T3 Task performance & $6.32(.74)$ & -.20 & $.27^{*}$ & .09 & $.20^{*}$ & & \\
\hline 6 & T3 P. Turnover Intentions & $1.83(.98)$ & $.26^{* *}$ & -.04 & $-.29^{* *}$ & -.15 & $-.26^{*}$ & \\
\hline 7 & T3 Retirement Intentions & $2.48(1.31)$ & .10 & -.18 & -.13 & .13 & .04 & .14 \\
\hline
\end{tabular}


Chapter 4: Linking Perceived Work Ability to Supervisor-Rated Job Performance

Following Study 1 and Study 2, one of the remaining questions in the WA literature is the extent to which WA - and PWA specifically - is related to supervisor ratings of job performance. That is, a key issue identified in the comprehensive metaanalytic investigation of the WA construct is that WA has not been linked to supervisor ratings of job performance. Although both Study 1 and Study 2 provide evidence that PWA is related to self-report ratings of performance, and thus, may also be related to supervisor rating of performance, that relation needed to be empirically explored. Thus, Study 3 (Chapter 4 of this dissertation) sought to provide evidence that PWA is indeed related to two aspects of job performance, task performance and creative performance.

As workers continue to age and the workforce becomes more age-diverse, promoting work ability (WA) will hold great importance for individuals, organizations, and society (Cadiz, Brady, Rineer, \& Truxillo, 2019; McGonagle et al., 2015). Yet in the United States, WA research and its use in practice has not caught on as quickly as it has in other countries (e.g., European nations; Bethge et al., 2012; Camerino et al., 2008; Ilmarinen et al., 1997; von Bonsdorff et al., 2011). From the perspective of US employers, this may be due in part to a focus on outcomes such as job performance and profitability and skepticism about whether WA affects these outcomes. Specifically, although research has linked WA to important long-term outcomes such as retirement and disability (Sell et al., 2009; von Bonsdorff et al., 2011), and to attitudinal variables, such as job satisfaction (Agnew et al., 2015; Camerino et al., 2008) and organizational commitment (Hakanen et al., 2008; McGonagle et al., 2015), until recently (see Von 
Bonsdorff et al., 2018 for an exception) researchers had only established links between WA and self-report ratings of job performance (Zacher \& Rudolph, 2017, Wagenaar et al., 2013). Von Bonsdorff and colleagues (2018) demonstrated that at the company level, age was related to executive perceptions of company performance through perceptions of work ability (PWA). This step toward establishing the link between PWA and performance is critical. However, as this area of research garners additional attention, it is increasingly important to a) establish evidence that PWA is related to other facets of performance and b) identify the extent to which PWA is related to performance across the lifespan.

Drawing upon the Job Demands-Resources model (JD-R), the present study will address these research gaps and contribute to the literature in a number of ways. First, this study evaluates the links between PWA and three performance indicators as rated by supervisors; engagement, task performance, and creative performance. Second, this study explores the extent to which the relations between PWA and each of the performance indicators remains consistent across the lifespan. Third, this study will more firmly place PWA within the JD-R framework by linking it to performance outcomes, which are commonly considered the outcomes of interest when investigating the JD-R model and of utmost importance to organizations.

\section{Hypothesis Development}

\section{Job Demands-Resources Model}

In line with the JD-R model, the mediating mechanisms engagement, burnout, and as I argue, PWA, stem from greater levels of job and personal resources and lower levels 
of job demands (Bakker \& Demerouti, 2007, 2011; Demerouti et al., 2001). That is, strain results from having demands that are in excess of the available resources to meet such demands, whereas motivation results primarily from having a sufficient levels of resources. As demonstrated in Study 1, research on PWA supports PWA being viewed as a mediating mechanism which is negatively related to job demands and positively related to job and personal resources. Subsequently, PWA can lead to workplaces outcomes that are negative (e.g., turnover; Rongen et al., 2014) when PWA is low, as well as positive (e.g., high job satisfaction; McGonagle et al., 2015; Wagenaar et al., 2013) when PWA is high. However, one of the specific outcomes posited by the JD-R is job performance, which is positively related to motivation and negatively related to strain (Demerouti et al., 2001. Bakker \& Demerouti, 2007, 2011). Initial research suggests that PWA may also be related to performance, however, as noted above, research on PWA and performance is in its nascent stage.

Performance Research

The notion that PWA should be strongly related to performance is supported by other areas of research as well. For example, research on performance (Campbell, McCloy, Oppler, \& Sager, 1993), job analysis (Cornelius, Carron, \& Collins, 1979; Dierdorff \& Morgeson, 2007; Levine, Ash, Hall, \& Sistrunk, 1983; Morgeson, \& Dierdorff, 2011), selection (Ryan \& Ployhart, 2014), training (Baldwin \& Ford, 1988; Blume, Ford, Baldwin, \& Huang, 2010; Salas, Tannenbaum, Kraiger, \& Smith-Jentsch, 2012) and individual abilities (Arneson, Sackett, \& Beatty, 2011) all suggest that the individual knowledge, skills, and abilities are important for understanding who will 
perform well and succeed in a given role. As such, ratings of PWA, which is defined as an individual's perceptions of their ability to meet the demands of their job (Ilmarinen et al., 1991; 1997), should be positively related to performance. That is, if individuals accurately rate their PWA, they feel that they have the necessary ability accomplish their work tasks. Although self-report ratings are subject to potential rating errors (Kruger \& Dunning, 1999; Sheldon, Dunning, \& Ames, 2014), this potential drawback can also be argued for a wide range of psychosocial variables, which are commonly evaluated as selfperceptions. Further, as demonstrated in previous research, PWA is related to other important organizational outcomes (e.g., retirement, disability status, job satisfaction; see Cadiz et al., 2018, Study 1 for a qualitative and quantitative review). Put simply, if an individual rates themselves as having poor PWA, and thus being unable to meet the demands of their job, it is unlikely that they will be rated as a high-performing employee. Importantly, to investigate the PWA to performance relation, it is also important to acknowledge that job performance is not a unidimensional outcome. Researchers have long delineated between task performance and organizational citizenship behaviors (Motowidlo \& Van Scotter, 1994), and since then, a host of other specific performance indicators have been acknowledged as important for organizations to consider (e.g., proactive behaviors, leadership behaviors; see Campbell \& Wiernik, 2015 for a review). As the nature of work shifts to be more dynamic and flexible (Morgeson \& Dierdorff, 2011; Spreitzer, Cameron, \& Garrett, 2017) the consideration of multiple performance indicators to adequately sample the criterion domain are critical to understanding the 
antecedents to specific aspects of performance. This study evaluates the relations between PWA and supervisor ratings of engagement, task performance, and creativity.

Supervisor-rated engagement. A key mediating mechanism within the JD-R framework is employee engagement (Demerouti et al., 2001. Bakker \& Demerouti, 2007, 2011). As demonstrated in Study 1 and empirical research (e.g. Airila et al., 2014; Airila, Hakanen, Punakallio, Lusa, \& Luukkonen, 2012), PWA is generally positively related to engagement. However, engagement is almost always measured from the perspective of the employee, as opposed to the perspective of supervisors. Supervisor ratings of engagement may therefore yield different pieces of information compared to self-report ratings. For example, engagement often refers to internal energy, such as vigor, dedication, and absorption (Schaufeli, Bakker, \& Salanova, 2006) or the extent to which individuals employ and express themselves at physically, cognitively, and emotionally at work (Kahn, 1990) which may not be visible to a supervisor. Yet a supervisor perceiving an employee as engaged may trigger downstream supervisor behaviors (e.g., provision of challenging work assignments) that are important for employee performance, whereas self-report ratings of engagement may not necessarily translate to observed engagement by one's supervisor. Thus, evaluating the relation between PWA and supervisor ratings of performance is important. I argue that higher levels of PWA can increase engagement because having the requisite abilities necessary to perform job duties is a vital aspect of the extent to which individuals will stay engaged at work. That is, engagement can be considered the extent to which one employs and expresses themselves physically, cognitively, and emotionally at work (Kahn, 1990). As such, having the sufficient 
physical, mental, and emotional abilities to meet the demands of the job are critical for staying engaged at work. For example, if an individual does not have the necessary ability to perform their job duties, they would be unable to express their abilities at work and thus, likely to become frustrated, unmotivated and disengaged based on the conceptual meaning of engagement from Kahn (1990). Taken together, based on the JD$\mathrm{R}$ framework as well as previous research linking PWA to self-rated engagement, I hypothesize that:

Hypothesis 1: PWA will be positively related to supervisor ratings of engagement. Task performance. In addition to the relation between PWA and engagement, additional research is needed to more firmly establish the link between PWA and supervisor ratings of task performance. Task performance refers in role behaviors that serve as a core part of one's job and that benefit the organization (Campbell, 1993; Campbell \& Wiernek, 2015; Griffin, Neal, \& Parker, 2007). As such, task performance is a key organizational outcome and among the most important for organizations to consider. PWA has been shown to be related to self-report ratings of performance (Zacher \& Rudolph, 2017), and more recently, Von Bonsdorff and colleauges (2018) demonstrated that PWA was positively related to CEO evaluations of company performance. This finding is critical in establishing the business case for PWA being important to organizations, yet only addresses company performance from the perceptions of the CEO, as opposed to more direct supervisor ratings of in-role performance. Thus, although the research on PWA and performance is growing, thus far the research has generally focused on self-report ratings and not sufficiently investigated 
the boundary conditions for the PWA to performance relation. Despite the gaps in PWA to performance research, the available research evidence as well as theoretical underpinnings of the JD-R lead me hypothesize that:

Hypothesis 2: PWA will be positively related to supervisor ratings of task performance.

Creative performance. In contrast to more routine performance behaviors such as task performance, creative performance refers to ideas, products, or procedures that are novel and have some use for an organization (Amabile, 1996). Creative performance is argued to be important for organizational effectiveness and survival (Amabile, 1996), and as jobs become more dynamic in nature, creative performance will continue to be an important dimension of performance for organizations to monitor. Importantly, creative performance involves a degree of experimentation, working through challenging circumstances, and applying novel problem solving approaches (Amabile, 1983, 1988; Tierney \& Farmer, 2011). However, as noted by Amabile (1988) creative performance builds on existing content expertise. Thus, having the requisite WA - and thus PWA - to meet the demands of one's job would serve as a necessary condition to engage in creative performance. For example, if an individual possess the capacity to adequately perform their basic job requirements, the JD-R model suggests that individuals will then have a greater amount of available resources to engage in behaviors that are nonconforming, see problems from new and insightful perspectives, and ultimately, engage in more creative behaviors. On the contrary, those with low levels of PWA may be struggling to meet the bare minimum requirements at work, and thus, not have the capacity to engage in 
additional creative behaviors. Given the preceding arguments, I posit that PWA will be positively related to creative performance.

Hypothesis 3: PWA will be positively related to supervisor ratings of creative performance

PWA and Performance across the Lifespan

Given the societal shift to an older and more age-diverse workforce, the influence of age as a boundary condition on the relations between performance and its antecedents is increasingly important to consider. As proposed in Hypotheses 1-3, PWA is expected to be related to engagement and performance. However, as demonstrated metaanalytically in Study 1, PWA is also negatively related to age, that is, as workers age they generally experience declines in PWA (e.g., Ilmarinen et al., 1991a, 1991b, 1997).

Further, there is a great deal of research suggesting that as people age, their motivations, skillsets, and even personality change (Baltes, 1987, 1999; Heckhausen, Wrosch, \& Schulz, 2010; Sameroff, 2010). Drawing on Sameroff's (2010) unified theory of development, individuals are more similar to one another at younger ages and diverge in their capacities later in life. As such, the working capacity of older workers may be more varied than the working capacity of younger workers, and thus, sustained high levels of PWA may be particularly important later in life. As potential evidence of the moderating effects of age, age has been shown to moderate the effect of stressors (Shirom, Shechter, Gilboa, Fried, \& Cooper, 2008) and resources (Yaldiz, Truxillo, Bodner, \& Hammer, 2019) such that the influence of stressors and resources on outcomes can vary across the lifespan. Tying this evidence together, I hypothesize that PWA will be a stronger 
determinant of performance among older workers. That is, as workers age, PWA will become increasingly important for success in the workplace.

Hypothesis 4: Age will moderate the relations between PWA and engagement, task performance, and creative performance, such that PWA will be more strongly related to performance among older workers.

\section{Method}

In order to assess the proposed hypotheses, data were collected at a healthcarerelated organization on the West Coast of the United States. Data were collected from organizational records, employee self-report surveys, and supervisor rating forms. In total, the data were collected from employees and supervisors across a three-month time period to maximize participation in the survey. Participants were not compensated by the research team but were provided time and available space during their work hours to complete the survey, and provided the survey link to complete the survey at home if desired. The researchers and organization leaders also informed participants that their individual responses would not be shown to organization stakeholders, and that data would only be presented to the organization stakeholders at the aggregate level. Sample

In total, 268 employees completed the employee portion of the survey. Performance ratings were obtained from 89 supervisors rating 375 employees. The final sample of matched surveys included 215 individuals who completed the WA rating scale and had their supervisor complete their performance rating form. Employee age was available from all employees through organizational records. The sample consisted of 
employees in administrative (52.2\%) and healthcare-related (e.g., physical therapist) positions $(47.8 \%)$. The average sample age was $37.29(S D=11.28)$.

Measures

Perceived work ability. Perceived work ability (PWA) was measured with a fouritem scale used by McGonagle and colleagues (2014, 2015). This scale uses three items from the original Work Ability Index (WAI; Morschhäuser \& Sochert, 2007) including: "How would you rate your current work ability compared to your lifetime best?" and an additional item that is particularly relevant in this sample, "Rate your work ability in relation to the social/interpersonal demands of the job." The first item, current WA compared to your lifetime best, is rated on a 0 (completely unable to work) - 10 (work ability at its lifetime best) point scale, and the remaining items are rated on a $1-5$ scale. Higher scores indicate greater PWA, and the scale demonstrated acceptable internal consistency in this sample $(\alpha=.79)$.

Supervisor rated engagement. Supervisor ratings of engagement were provided with three items scored on a 1 (strongly disagree) to 5 (strongly agree) scale, with higher scores indicating greater levels of engagement. This scale was developed for the present study. A sample item is "seems to be engaged in his/her work." However, the scale demonstrated less than optimal internal consistency $(\alpha=.65)$.

Job performance. Job performance as rated by supervisors was assessed as task performance and creative performance. Each scale was scored on a 1 (strongly disagree) to 5 (strongly agree) scale, with higher scores indicating greater performance ratings. Task performance was assessed with five items from Podsakoff and MacKenzie (1989) 
regarding the core duties of the employees' job. A sample is "Meets all the formal performance requirements of the job.” The task performance scale demonstrated good internal consistency $(\alpha=.87)$. Creative performance was evaluated with six items from Tierney, Farmer, and Graen (1999). A sample item is "tries out new ideas and approaches to work problems." This scale demonstrated excellent internal consistency $(\alpha=.94)$.

\section{Analytic Strategy}

In order to evaluate the proposed hypotheses, structural equation modeling (SEM) in MPlus version 8.1 was used. Specifically, for PWA and each outcome, latent variables were constructed using observed items. To account for the nested structure of the data, that is, employees are nested within supervisors, the complex modeling in MPlus was used. The complex model was chosen as opposed to the two-level model because the between-group variance attributed to the supervisors was not of primary interest for this research study. However, it was necessary to control for the nonindependence of supervisor ratings. In total, there were 89 supervisors and an average of 2.42 employees per supervisor.

Prior to conducting hypothesis testing, I assessed the measurement model for model fit. I then evaluated the standardized coefficients from the SEM model in line with the proposed hypotheses. The model used to test Hypotheses 1-3 included each PWA item loading onto a latent factor, each performance item loading onto its latent factor, and age entered as a control variable. To evaluate the proposed interaction between PWA and age on supervisor ratings of engagement and performance, I modeled the effect of the age by latent PWA variable using the XWITH command in MPlus. These steps are in line 
with the recommendations from Maslowsky, Jager, and Hemken (2015) for assessing latent variable moderators as well as recommendations from Muthen (2011) who specified that "You can use the XWITH option to estimate an interaction between a latent and observed variable." As such, the final model, which was used to evaluate Hypothesis 4, included the three latent performance-related variables which were regressed on the latent PWA variable, the observed variable age, and the moderating effect of PWA by age. All analyses were conducted using full information maximum likelihood (FIML). FIML allows estimation of missing data under the assumption of missing at random (MAR; Little \& Rubin 1987). This approach has been used in numerous research articles in top-tier journals in the organizational sciences (e.g., Koopmann, Lanaj, Wang, Zhou, \& Shi, 2016; Schaubroeck et al. 2018).

\section{Results}

Prior to evaluating the hypotheses tests, I first evaluated the fit of the model specified for Hypotheses 1-3. I specified a four-factor model with the latent variables 1) PWA (employee-rated) and supervisor ratings of 2) engagement, 3) task performance, and 4) creative performance, and their respective observed items loading onto each latent factor. The fit indices of this model were excellent, RMSEA $=.027, \mathrm{CFI}=.986, \mathrm{CLI}=$ $.983 \mathrm{SRMR}=.045$, suggesting that the measurement model fit the data well. However, although the model fit was excellent, one item did not load well onto the specified latent factor. A reversed coded item for the supervisor rated engagement scale loaded onto its latent factor poorly (.347). Indeed, this item was also part of the scale, which demonstrated the lowest level of internal consistency. Given this poor loading, I 
conducted analyses with the item included and dropped. The substantive conclusions were not influenced by retaining or dropping the poorly loading item. Thus, the following results include the poorly loading item.

After establishing that the measurement model provided acceptable fit to the data, the hypotheses were evaluated. To test Hypothesis 1, that PWA would be positively related to supervisor rated engagement, the standardized path from the latent PWA factor to the latent engagement factor was evaluated. The link between PWA and supervisorrated engagement was not significant, $p=.277$, and thus, Hypothesis 1 was not supported. To evaluate Hypothesis 2, the standardized path from the latent PWA factor to the latent task performance factor was evaluated. In support of Hypothesis 2, PWA was positively related to task performance, $\beta=.184, p=.022$. To evaluated Hypothesis 3 , that PWA would be positively related to creative performance, the standardized coefficient between the latent PWA and latent creative performance variables was evaluated. In support of Hypothesis 3, PWA was positively related to creative performance, $\beta=.159, p$ $=.029$. Taken together, these results provide additional evidence of the link between PWA and performance, while also demonstrating a potential inconsistency in the relation between PWA and engagement when engagement is not self-reported.

Hypothesis 4 posited that in the effects of PWA on each performance indicator would be moderated by employee age. To test this hypothesis, a second SEM model was constructed with the latent PWA variable, observed age variable, and the interaction term between the two included in the model. Regarding supervisor rated engagement and task performance, there was no moderating effect of age on the relation between PWA and 
engagement $(p=.409)$ and task performance $(p=.958)$. Similarly, the moderating effect between PWA and creative performance was not statistically significant $(\beta=.211, \mathrm{p}=$ .076) see Figure 6. As can be seen in Figure 6, descriptively, the creative performance of younger workers seemed to be unaffected by PWA. However, for older workers, when reporting low PWA they were rated as engaging in lower levels of creative behaviors than older workers with high levels of PWA.

Supplemental Findings

Beyond the hypothesized relations, I also conducted some follow-up analyses. When evaluating the direct effect of age on performance, a negative relation between age and creative performance was observed $(\beta=-.198, p<.01)$. However, the relations between age and supervisor ratings of engagement $\beta=.053, p=.301$ and task performance $\beta=.001, p=.967$ were not significant. This suggests that supervisors rated older workers as being less creative, but no less likely to perform well in terms of their engagement at work or their performance.

Given that the employees in the present study worked in two distinct contexts (i.e. administrative vs client-facing), I replicated the hypothesis tests from Hypotheses 1-4 with job type as a control variable. In each case, job type was not significantly related to the outcome variables. Further, when controlling for job type, there were no changes to the substantive conclusions of the hypothesis tests.

Discussion

The present study evaluated the link between PWA and supervisor ratings of engagement, task performance, and creativity. In addition, this study evaluated the 
moderating role of age on the links between PWA and each performance-related correlate. Overall, support was found for the link between PWA and two dimensions of performance (i.e., task performance and creativity). This supports the notion that PWA is indeed an important variable for organizations to consider with regard not only to longterm outcomes, but to performance as well. Interestingly, the link between PWA and supervisor rated engagement was not supported. This is at odds with previous literature which has consistently linked PWA to engagement when using self-report instruments. This may be due to the fact that engagement as a construct may not be as observable to others, and by definition is a type of self-assessment. Finally, this study found minimal evidence of the moderating role of age on the PWA to performance relations. That is, of the three potential moderating effects, only one of them approach being statistically significant. That said, the marginal effect and associated line plots do point to an interesting and potentially important point to consider, namely, that PWA relates to the creative performance of older workers more than it does for younger workers. Taken together, these findings make important practical and theoretical contributions to the literature.

Practical Contributions

As noted in the opening sections of this chapter, research on PWA in the U.S. has generally lagged behind research on PWA in other nations. However, I believe that this lack of research interest in the U.S. may largely be a function of the more health and well-being types of outcomes commonly investigated in relation to PWA. This may be due in part to a greater emphasis on the antecedents to performance and profit-based 
outcomes in the U.S. relative to the wellbeing outcomes emphasized in Europe (e.g., retirement, future disability status). In addition, a reduced interest in WA research in the U.S. may be due to the fact that compared to many European countries, there is less focus on the aging workforce in the U.S., as the aging trends in the U.S. are less acute.

Therefore, establishing the link between PWA and performance, as I do in the present study, is critical to promote the adoption of more research investigating PWA. This study thus takes an important step toward that goal, and with the findings of this study linking PWA to performance, organizations may be more likely to view supporting PWA across the lifespan as an important organizational practice.

Although it did not reach statistical significance, the interaction of PWA and age on creative performance, as well as the significant but non-hypothesized negative relation between age and creative performance, are worth discussing. There is a stereotype that older workers are unwilling to change or learn new skills (Ng \& Feldman, 2012), despite generally being unsupported empirically ( $\mathrm{Ng} \&$ Feldman, 2013). If it were accurate, that negative stereotype would suggest that the relation between age and creativity should be negative. Indeed, in this sample, it was. Putting aside the potential that this finding could be due in part to biases held by supervisors themselves, the moderating effect points to an interesting underlying interpretation. When workers reported high PWA, there were minimal differences in creative performance among younger and older workers. In fact, under conditions of high PWA, older workers were rated as engaging in slightly more creative behaviors than younger workers. However, when low PWA was reported, older workers were rated much lower on creative performance, whereas younger workers were 
rated essentially the same on creative performance regardless of their PWA. This points to a need for more research attention in this area, but also provides at least some evidence that the promotion of PWA later in life may be particularly important not only for delaying retirement but for important facets of performance for organizations.

Theoretical Contributions

Although a large body of research regarding WA and PWA has accumulated, PWA has only recently been situated with a theoretical framework, the JD-R model. Due in part to this recent integration, the final link between PWA and job performance has only been established in a handful of empirical studies. Further, nearly all of the existing research on PWA and performance has been conducted using self-report ratings of performance, and no previous research has demonstrated the relations of PWA to supervisor ratings of task performance, and creative performance. As such, the present research makes a theoretical contribution by linking PWA to supervisor ratings of task and creative performance, which more firmly plants PWA as a construct within the JD-R framework. This theoretical contribution supports future research investigating the links between PWA and other facets of performance, as well as testing the entire mediating process posited by the JD-R model.

\section{Limitations and Future Directions}

Although this study has several strengths, it also has its limitations. First, the sample was relatively young compared to many work samples, which may have inhibited the ability to detect age-related interactions. Although this is a concern, it remains notable 
that one interaction effect nearly reached statistical significance. Further, the age group one standard deviation above the mean, which is operationalized as the "older workers" in this study, was nearly 50 years old. Future research should investigate the role of PWA in terms of performance among relatively older samples of workers to further clarify the moderating effect of age on the PWA to performance relations.

A second limitation of the present study is that although this study establishes a link between PWA and two indicators of performance, a host of other facets of performance have yet to be examined. For example, this study did not include a measure of organizational citizenship behaviors, adaptive behaviors, teamwork, leadership, or other important aspects of performance. As the nature of work evolves, the ability to work in teams, be adaptive, and engage in organizational citizenship behaviors will become increasingly important. As such, this study provides initial evidence of the link between PWA and performance, yet future research should continue to elaborate on such findings.

Finally, although this study draws on the JD-R model to establish its hypotheses, it does not test PWA as a mediating mechanism explicitly. Thus, it is fair to question whether or not PWA would be observed as a mediator linking job resources and demands to performance outcomes. Although this is certainly a limitation, the direct relation of PWA to performance demonstrated in this study represents the final path posited in the JD-R model, and as such, does make an important theoretical and practical contribution to the literature. Future research should more thoroughly investigate the mediated process 
posited by the JD-R model and continue to explore moderators to the PWA to performance relations.

\section{Conclusion}

In this study I set out to evaluate a relatively straightforward, but important, set of hypotheses that link PWA to supervisor ratings of engagement, task performance, and creativity, and investigate the moderating role of age. Support was found for the relations between PWA and task and creative performance, further establishing PWA within the JD-R framework as being related to performance-based organizational outcomes. This finding provides substantial evidence for the use of PWA by organizations to not only monitor risk for retirement, but to promote critical proximal outcomes, such as task and creative performance. Follow-up analyses revealed that within this sample, age was negatively related to creative performance. However, moderator analysis and plot diagrams suggested that this effect may be because older workers who reported low levels of PWA were rated as least creative, whereas older workers with high levels of PWA were rated as just as creative as their younger counterparts. Taken together, these findings provide strong evidence for the importance of considering PWA in performance outcomes and in doing so, provide additional evidence for the utility of employee PWA for organizations. 
Table 11.

Means, Standard Deviations, and Zero Order Correlations among Study 3 Variables.

\begin{tabular}{|c|c|c|c|c|c|c|c|}
\hline & Variable & Mean (SD) & 1 & 2 & 3 & 4 & 5 \\
\hline 1 & Client Facing Job & $47.8 \%$ & & & & & \\
\hline 2 & Age & $36.78(11.00)$ & $.17 * *$ & & & & \\
\hline 3 & PWA & $4.42(.56)$ & -.02 & .05 & & & \\
\hline 4 & Engagement & $4.26(.62)$ & $.14 * *$ & -.04 & .08 & & \\
\hline 5 & Task Performance & $4.54(.57)$ & .05 & .01 & $.15^{*}$ & $.59 * *$ & \\
\hline 6 & Creativity & $4.00(.82)$ & .02 & $-.20 * *$ & $.15^{*}$ & $.49 * *$ & $.49 * *$ \\
\hline
\end{tabular}




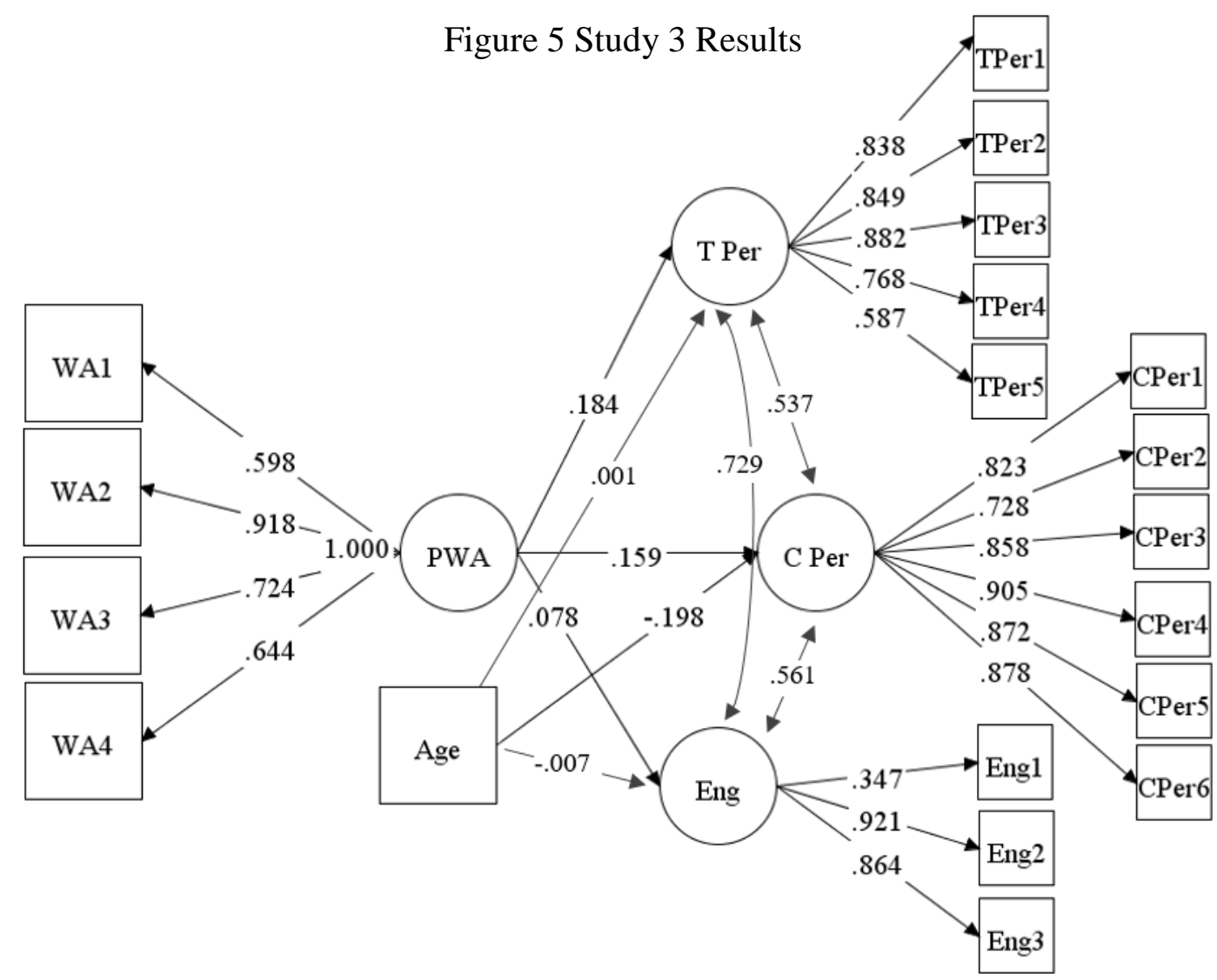

Figure 5. SEM results for Hypotheses 1-4. Testing the relations between PWA and engagement, task performance and creativity. Age entered as a control variable. 
Figure 6 Study 3 Moderation Plot

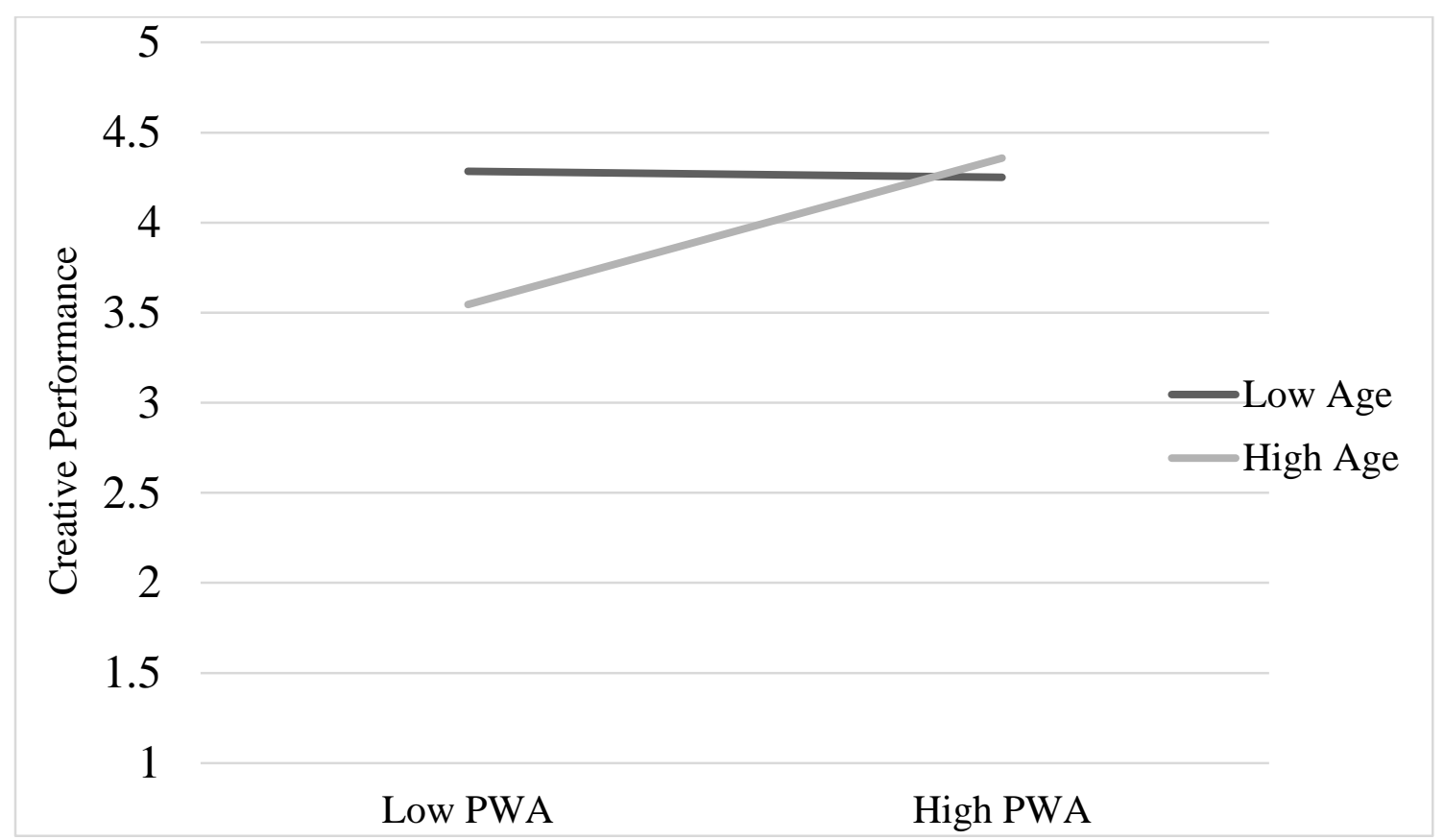

Figure 6. Interaction plot showing the moderating effect of age on the relation between PWA and creativity. 
Chapter 5: General Discussion

Society and organizations are facing an unprecedented shift to an older and more age-diverse workforce, which will be the "new normal" for the foreseeable future. To address these challenges, organizational practices and societal policies that support people working later in life are critical. Specifically, keeping employees motivated and able to work effectively across the lifespan will be critical for softening the economic and societal costs associated with a large number of workers reaching retirement age.

Research on work ability (WA) and perceived work ability (PWA) is well positioned to help address these issues, inform interventions, and serve as a tool for organizations to better support workforce participation across the lifespan. However, despite the promise of WA research and the imminent shift to an older workforce, the WA concept has yet to be fully adopted within the organizational sciences, and thus, its utility has yet to be fully realized. Together, the three studies of this dissertation highlight the value of assessing WA and PWA, and in doing so, advance WA theory and make substantial practical contributions to the literature.

Summary of Results

First, Study 1 provides a quantitative synthesis of 247 studies and 312,987 participants, supplemented by a sample of online MTurk participants. Specifically, Study 1 identified the theoretical antecedents of WA as well as its attitudinal, wellbeing, and behavioral outcomes. Overall, the results indicated that health was among the most strongly related correlates to WA, and that other personal resources (e.g., positive personality traits) and organizational factors (e.g., job demands, job resources) are also 
related to WA. This synthesis serves as a critical bridge between the medical literature, where WA research originally developed, and the organizational sciences, where the concept of WA is now gaining traction.

Study 1 also demonstrates how occupation type influences the nomological network of WA. Specifically, in several instances, correlates of WA were more strongly related to WA among white collar workers compared to blue collar workers (e.g., job resources, job control, strain) whereas health behaviors were more strongly related to WA among blue collar workers compared to those in nursing occupations (e.g., overall health behaviors, smoking). These differences have important implications for how organizations and practitioners can tailor interventions targeting the maintenance of WA. In addition, Study 1 provides evidence that measures of PWA retain a great deal of the predictive power associated with the WAI, but that in certain cases the WAI is more strongly related to the correlate of interest. Specifically, when investigating general health, current health status, and future disability status, the WAI was more strongly related to the correlate of interest than measures of PWA. Thus, in certain situations, the WAI does provide greater explanatory power, albeit at a substantially greater cost to participant time, invasiveness, and resources.

The results from Study 1 and Study 2 suggest that PWA is a unique construct, which correlates with established constructs (e.g., perceived fit, job self-efficacy, and general self-efficacy), but is unique in its own right. Specifically, Study 1 demonstrated that PWA can explain unique variance in outcomes of interest (e.g., engagement, burnout, task performance, disability intentions, and health) beyond the established 
constructs general self-efficacy, job self-efficacy, and perceived fit. Moreover, Study 2 found that PWA can serve as a mediating mechanism between age discrimination and specific outcomes (e.g., task performance, presenteeism, life satisfaction, engagement, stress) even when accounting for perceived fit. Indicating that PWA is important to consider, even when accounting for related and established psychosocial constructs.

Study 2 also demonstrated that age discrimination is an interpersonal antecedent of WA. As the workforce becomes older and more age-diverse, the results of Study 2 suggest that reducing age discrimination will play an important role in supporting workers across the lifespan. Moreover, Study 2 found evidence that the negative effect of age discrimination on PWA has downstream consequences as well. Specifically, results of Study 2 suggest that age discrimination is negatively related to PWA and that age discrimination has indirect negative effects on a range of occupational outcomes (e.g., task performance, retirement intentions, engagement, and burnout).

Finally, each of these studies expands upon the link between WA and job performance. As identified in Study 1, no studies have linked supervisor ratings of individual job performance to WA. However, self-report rating of PWA have been linked to job performance and Study 1 found evidence of this relation through meta-analysis. Study 2 provided additional evidence of the link between PWA and self-report ratings of job performance, whereas Study 3 filled a much-needed gap by linking PWA to supervisor ratings of task and creative performance. Linking PWA to supervisor ratings of performance was crucial because it helps provide a strong case for the use of PWA to 
organizations as a tool to monitor their organizational change initiatives and effectively support their workforce, while still realizing more immediate performance gains. Theoretical Contributions

The WA construct was initially developed in an atheoretical manner in the medical literature (Ilmarinen et al., 1991a, 1991b), but due in part to its utility for predicting retirement and future disability status, has recently been drawn upon by researchers in the organizational sciences (e.g., Airilia et al., 2012, 2013; McGonagle et al., 2014, 2015). However, this adoption also led to silos of WA research, culminating in the lack of a consistent definition and operationalization of the construct. Together, Studies 1-3 of this dissertation advanced WA theory by more concretely grounding WA and PWA within a scientifically accepted theory, the JD-R model. In doing so, these studies provide a succinct definition of WA as "an individual's ability to meet the requirements of their job" and provides evidence that PWA can be considered an appropriate measure of WA. More clearly defining the WA construct will help future WA research by ensuring that researchers across disciplines are assessing the same overarching construct, and therefore, help ensure that research findings can be generalized across disciplines.

A second theoretical contribution made by the present dissertation was to demonstrate that PWA is indeed a unique construct that remains important when considering other psychosocial constructs (e.g., job self-efficacy, general self-efficacy, and perceived fit). Findings from all three studies indicate that PWA is more important for in-role behaviors (i.e. task performance), whereas perceived fit, for example, appears 
to be more important for job attitudes (e.g., job satisfaction). Thus, the findings of this dissertation indicate that PWA should be utilized in conjunction with theoretically related constructs (e.g., perceived fit, job self-efficacy, and general self-efficacy) as each may relate to organizational outcomes in unique ways. The evidence that PWA is a unique construct that is useful for explaining variance in organizational outcomes should shape future theoretical research on PWA. Specifically, future theoretical research should focus on the specific instances in which job self-efficacy, perceived fit, and PWA may be most important to consider, and focus on understanding the boundary conditions of such relations.

A third theoretical contribution is the identification of PWA as a mediating mechanism between age discrimination and workplace outcomes (e.g., task performance, retirement intentions, engagement, and stress). Specifically, couching age discrimination within the JD-R framework as a hindrance demand provides additional theoretical justification for the overall model presented in Study 1, which posits that the primary role of PWA is as a mediator between job demands, job resources, and personal resources, and outcomes of interest. Previously, age discrimination research focused on justice as the mechanism through which age discrimination leads to poor outcomes. However, evidence from Study 1, which demonstrated the positive relation between justice perceptions and WA, and evidence from Study 2, which found a negative link between age discrimination and PWA, indicate that unfair treatment may influence outcomes through other mechanisms as well. Specifically, persistent age discrimination may erode workers' perceptions of their ability to do their job, and thus, negatively influence 
performance, engagement, and other important outcomes through PWA. Future theoretical research should focus on evaluating PWA as a mediating mechanism between unfair treatment and work and non-work outcomes.

Practical Contributions

In addition to the critical theoretical contributions that this dissertation makes, this dissertation makes a number of practical contributions as well. First, this set of studies suggests several avenues for researchers and organizations to focus on as intervention targets. Specifically, Study 1 suggests emphasizing the promotion of worker wellbeing, including physical activity and general, mental, and physical health, because health variables were most strongly related to WA and PWA. Second, ensuring fair interpersonal treatment also appears to be critical for maintaining WA. Both justice perceptions (Study 1) and age discrimination (Study 2) were shown to have substantial relations with PWA. Taken together, Study 1 and Study 2 indicate that organizations in which employees are treated fairly will also have a workforce with higher levels of PWA. Third, organizations must pay critical attention to both the outcomes they are interested in and the occupational demands when selecting the targets of the intervention. For example, the positive relation between job control and PWA was substantially stronger in white collar occupations compared to blue collar occupations, and thus, job control may be a less useful intervention target for blue collar workers.

A second practical contribution is that the present dissertation makes the "business case" for the use of PWA in organizational research. Indeed, each study demonstrated that PWA was related to at least one dimension of job performance. As 
noted in Chapters 1-4, research on WA within the United States has largely lagged behind WA research in European countries. This discrepancy may stem from the fact that the US is aging somewhat less rapidly than other nations and that WA has generally been studied with a focus on retirement, absenteeism, and disability status, as opposed to job performance, which may be of the greater concern among organizational researchers in the US. The combined evidence linking PWA to job performance (e.g., self-report ratings in Study 1, Study 2; supervisor ratings, Study 3), provides strong support for organizations monitoring PWA in order to promote employee performance across multiple domains (i.e. task performance, creativity). Limitations and Future Research Agenda Although the present studies make valuable contributions, there are also limitations to these studies, which point to important areas of future research. Overall, Study 1 represents a high volume of empirical research that is unmatched in the majority of organizational research. However, meta-analytic investigations inherently lack the fine-grain detail provided by rigorous and controlled empirical studies. Thus, additional research is needed to put into action the broad findings of Study 1, fine tune those findings, and make lasting contributions to the field. Specifically, to truly address the challenges associated with an aging workforce, researchers must put into action interventions that support the maintenance of WA across the lifespan. As such, future research should draw upon the findings of this dissertation to develop robust interventions targeting personal and organizational factors to promote WA across the 
lifespan, evaluate the results of those interventions, and fine-tune them to best support the aging and more age-diverse workforce of the future.

Agenda Item 1: Implement, evaluate, and fine-tune interventions based on the summary of relations between WA and its theoretical antecedents and outcomes.

Second, evidence from Studies 1-3 indicates that there is a great need to continue defining and developing the nomological network of PWA to a wider range of antecedents and outcomes. The vast majority of research on PWA and job resources and demands has focused on aspects of job control, support, or workload. However, characteristics of jobs are far more diverse than those characteristics alone. For example, research could consider job complexity, job feedback, or more creative oriented job requirements. Similarly, the amount of personal characteristics (e.g., personality traits) investigated in relation to PWA are minimal given the robust overall literature on personality and individual differences (Barrick \& Mount, 1991; Goldberg, 1990; Judge, Heller, \& Mount, 2002). Finally, interpersonal constructs, such as transformational leadership and other forms of discrimination are surprisingly absent from the WA literature. Although the WA construct itself has been studied for nearly 40 years, research on WA has only begun to take root in the organizational sciences in the past decade, and thus, the range of constructs considered in organizational research has not been fully explored in relation to WA. Thus, evidence from the present studies make substantial contributions, but also suggest that a great deal of research is still needed to better understand the nuances of the nomological network of WA. 
Agenda Item 2: Expand the nomological network of WA to include the breadth of individual differences and job characteristics represented in the organizational sciences in relation to WA.

Similar to the lack of research on the correlates of PWA, research identifying the boundary conditions of such relations is sorely needed. For instance, personality characteristics and other individual differences can serve as a boundary condition for the effect of job characteristics on outcomes of interest. That is, it remains to be seen if job demands and resources influence PWA in the same way for people with differing personalities, values, and individual traits. Similarly, research is needed to understand if PWA is predictive of its theoretical outcomes (e.g., retirement, absenteeism, job performance) across contexts, and the extent to which these relations are consistent among individuals with different underlying traits and dispositions.

Agenda Item 3: Identify the boundary conditions (i.e. moderating factors) between WA and its antecedents and outcomes.

Finally, given that the maintenance of PWA is viewed as important for successful aging, more research is needed on truly "older" samples of workers regarding PWA. Specifically, only a small number of studies have been conducted on samples of workers with a mean age of over 50. Workers in the U.S. are now required to work an additional 17 years after reaching age 50 in order be eligible for full retirement benefits, and the trend of later retirement ages will likely continue for the foreseeable future. Further, workers may remain in the workforce following their official retirement through bridge employment or other part-time work. Thus, understanding how to promote WA among 
this segment of the workforce will be critical to mitigating the economic impact of an aging workforce. Moreover, promoting WA among older workers will serve individuals well because those with the desire to continue working will be more capable of doing so.

Agenda Item 4: Conduct studies among workers at or nearing retirement age to evaluate changes in WA as workers enter retirement age.

\section{Conclusion}

The studies in this dissertation provide evidence that WA is well suited to address the forthcoming challenges associated with an aging and age-diverse workforce. The present research answers important and fundamental questions about WA as a construct, advance WA theory, and will help guide future theoretical and practical research on WA. Importantly, evidence from these studies suggests that the promotion of WA can be mutually beneficial to individuals and organizations. That is, promoting health and wellbeing provide clear benefits to individuals who as a function of being healthier are likely to have greater quality of life in addition to greater WA. In addition, the relations between WA and increased levels of performance, positive job attitudes, and later retirement ages benefit employers by providing increased productivity and a larger overall workforce. In sum, this multi-study dissertation makes clear that WA is a useful construct for individuals and organizations alike, and that it is well suited to address important research questions about the aging workforce and its societal implications in the coming decades. 


\section{References}

*Abdolalizadeh, M., Arastoo, A. A., Ghsemzadeh, R., Montazeri, A., Ahmadi, K., \& Azizi, A. (2012). The psychometric properties of an Iranian translation of the Work Ability Index (WAI) questionnaire. Journal of Occupational Rehabilitation, 22, 401-408.

*Abma, F. I., Brouwer, S., de Vries, H. J., Arends, I., Robroek, S. J., Cuijpers, M. P., \& van der Klink, J. J. (2016). The capability set for work: Development and validation of a new questionnaire. Scandinavian Journal of Work Environment \& Health, 42(1), 34-42.

*Abma, F. I., van der Klink, J. J., \& Bültmann, U. (2013). The work role functioning questionnaire 2.0 (Dutch version): examination of its reliability, validity and responsiveness in the general working population. Journal of Occupational Rehabilitation, 23(1), 135-147.

*Agnew, L., Johnston, V., Ludvigsson, M. L., Peterson, G., Overmeer, T., Johansson, G., \& Peolsson, A. (2015). Factors associated with work ability in patients with chronic whiplash-associated disorder grade II-III: A cross-sectional analysis. Journal of Rehabilitation Medicine, 47, 546-551.

*Ahlstrom, L., Grimby-Ekman, A., Hagberg, M., \& Dellve, L. (2010). The Work Ability Index and single-item question: Associations with sick leave, symptoms, and health-a prospective study of women on long-term sick leave. Scandinavian Journal of Work, Environment \& Health, 36, 404-412. 
*Airila, A., Hakanen, J., Punakallio, A., Lusa, S., \& Luukkonen, R. (2012). Is work engagement related to work ability beyond working conditions and lifestyle factors?. International Archives of Occupational and Environmental Health, 85, 915-925.

*Airila, A., Hakanen, J. J., Luukkonen, R., Lusa, S., \& Punakallio, A. (2013). Positive and negative mood trajectories and their relationship with work ability, self-rated health, and life satisfaction: A 13-year follow-up study. Journal of Occupational and Environmental Medicine, 55, 779-785.

*Airila, A., Hakanen, J. J., Schaufeli, W. B., Luukkonen, R., Punakallio, A., \& Lusa, S. (2014). Are job and personal resources associated with work ability 10 years later? The mediating role of work engagement. Work \& Stress, 28, 87-105.

*Alavinia, S. M., De Boer, A. G. E. M., Van Duivenbooden, J. C., Frings-Dresen, M. H. W., \& Burdorf, A. (2009). Determinants of work ability and its predictive value for disability. Occupational Medicine, 59, 32-37.

*Alavinia, S. M., van Duivenbooden, C., \& Burdorf, A. (2007). Influence of work-related factors and individual characteristics on work ability among Dutch construction workers. Scandinavian Journal of Work, Environment \& Health, 33, 351-357.

Amabile, T. M. (1983). The social psychology of creativity: A componential conceptualization. Journal of Personality and Social Psychology, 45(2), 357-368.

Amabile, T. M. (1988). A model of creativity and innovation in organizations. Research in Organizational Behavior, 10(1), 123-167. 
Amabile, T. M. 1996. Creativity and innovation in organizations (Vol. 5). Boston: Harvard Business School.

*Arandjelovic, M., Nikolic, M., \& Stamenkovic, S. (2010). Relationship between burnout, quality of life, and work ability index - directions in prevention. The Scientific World Journal, 10, 766-777.

Armstrong-Stassen, M., \& Schlosser, F. (2008). Benefits of a supportive development climate for older workers. Journal of Managerial Psychology, 23(4), 419-437.

Arneson, J. J., Sackett, P. R., \& Beatty, A. S. (2011). Ability-performance relationships in education and employment settings: Critical tests of the more-is-better and the good-enough hypotheses. Psychological Science, 22(10), 1336-1342.

*Assunção, A. A., Sampaio, R. F., \& Nascimento, L. (2010). Actions in small companies to promote occupational health: the case of the food and beverage sector. Brazilian Journal of Physical Therapy, 14, 52-59.

*Attarchi, M., Ghaffari, M., Abdi, A., Mirzamohammadi, E., Seyedmehdi, S. M., Rahimpour, F., Fazlalizadeh, M., \& Mohammadi, S. (2014). Assessment of the Relationship between Physical Working Conditions and Different Levels of Work Ability. Global Journal of Health Science, 6, 213-220.

Bakker, A. B., \& Demerouti, E. (2007). The job demands-resources model: State of the art. Journal of Managerial Psychology, 22, 309-328.

Bakker, A. B., \& Demerouti, E. (2017). Job demands-resources theory: taking stock and looking forward. Journal of Occupational Health Psychology, 22(3), 273-285. 
Bakker, A. B., Demerouti, E., \& Verbeke, W. (2004). Using the job demands-resources model to predict burnout and performance. Human Resource Management, 43(1), 83-104.

Bakker, A. B., Demerouti, E., Taris, T. W., Schaufeli, W. B., \& Schreurs, P. J. (2003). A multigroup analysis of the job demands-resources model in four home care organizations. International Journal of Stress Management, 10(1), 16-38.

Bakker, A. B., Schaufeli, W. B., Leiter, M. P., \& Taris, T. W. (2008). Work engagement: An emerging concept in occupational health psychology. Work \& Stress, 22(3), 187-200.

Baldwin, T. T., \& Ford, J. K. (1988). Transfer of training: A review and directions for future research. Personnel Psychology, 41(1), 63-105.

Baltes, P. B., Staudinger, U. M., \& Lindenberger, U. (1999). Lifespan psychology: Theory and application to intellectual functioning. Annual Review of Psychology, 50(1), 471-507.

Baltes, P. B. (1987). Theoretical propositions of life-span developmental psychology: On the dynamics between growth and decline. Developmental Psychology, 23(5), 611.

Baltes, P. B. (1997). On the incomplete architecture of human ontogeny: Selection, optimization, and compensation as foundation of developmental theory. American Psychologist, 52(4), 366-380. 
*Barnes-Farrell, J. L., Rumery, S. M., \& Swody, C. A. (2002). How do concepts of age relate to work and off-the-job stresses and strains? A field study of health care workers in five nations. Experimental Aging Research, 28(1), 87-98.

Barrick, M. R., \& Mount, M. K. (1991). The big five personality dimensions and job performance: a meta-analysis. Personnel Psychology, 44(1), 1-26.

Bayl-Smith, P. H., \& Griffin, B. (2014). Age discrimination in the workplace: identifying as a late-career worker and its relationship with engagement and intended retirement age. Journal of Applied Social Psychology, 44(9), 588-599.

Begg, C. B., \& Mazumdar, M. (1994). Operating characteristics of a rank correlation test for publication bias. Biometrics, 1088-1101.

*Beltrame, M. T., Magnago, T. S. B. D. S., Kirchhof, A. L. C., Marconato, C. D. S., \& Morais, B. X. (2014). Work ability in hospital housekeeping services and associated factors. Revista Gaucha de Enfermagem, 35(4), 49-57.

*Bennett, A. I., Hanley, J., Buckle, P., \& Bridger, R. S. (2011). Work demands during firefighting training: does age matter?. Ergonomics, 54, 555-564.

*Bertilsson, M., Vaez, M., Waern, M., Ahlborg Jr, G., \& Hensing, G. (2014). A prospective study on self-assessed mental well-being and work capacity as determinants of all-cause sickness absence. Journal of Occupational Rehabilitation, 25, 52-64.

*Bethge, M., \& Radoschewski, F. M. (2010). Physical and psychosocial work stressors, health-related control beliefs and work ability: Cross-sectional findings from the 
German Sociomedical Panel of Employees. International Archives of Occupational and Environmental Health, 83, 241-250.

*Bethge, M., \& Radoschewski, F. M. (2012). Adverse effects of effort-reward imbalance on work ability: Longitudinal findings from the German Sociomedical Panel of Employees. International Journal of Public Health, 57(5), 797-805.

*Bethge, M., Radoschewski, F. M., \& Gutenbrunner, C. (2012a). The Work Ability Index as a screening tool to identify the need for rehabilitation: longitudinal findings from the second German Sociomedical Panel of Employees. Journal of Rehabilitation Medicine, 44, 980-987.

*Bethge, M., Radoschewski, F. M., \& Gutenbrunner, C. (2012b). Effort-reward imbalance and work ability: cross-sectional and longitudinal findings from the Second German Sociomedical Panel of Employees. BioMed Central Public Health, 12, 1-9.

Bies, R. J., \& Moag, J. S. (1986). Interactional justice: Communication criteria of fairness. Research on Negotiation in Organizations, 1(1), 43-55.

Bilinska, P., Wegge, J., \& Kliegel, M. (2016). Caring for the elderly but not for one's own old employees? Organizational age climate, age stereotypes, and turnover intentions in young and old nurses. Journal of Personnel Psychology, 15(3), 95-.

Binnewies, C., Ohly, S., \& Niessen, C. (2008). Age and creativity at work: The interplay between job resources, age and idea creativity. Journal of Managerial Psychology, 23(4), 438-457. 
Blume, B. D., Ford, J. K., Baldwin, T. T., \& Huang, J. L. (2010). Transfer of training: A meta-analytic review. Journal of Management, 36(4), 1065-1105.

*Böerner, K., Scherf, C., Leitner-Mai, B., \& Spanner-Ulmer, B. (2012). Field study of age-differentiated strain for assembly line workers in the automotive industry. Work, 41 (1), 5160-5166.

*Bordin, G., Padalino, M. A., Perentaler, S., Castaldi, B., Maschietto, N., Michieli, P., \& Milanesi, O. (2015). Clinical profile and quality of life of adult patients after the fontan procedure. Pediatric Cardiology, 36, 1-9.

Borenstein M., Hedges L., Higgins J., Rothstein H (2009). Multiple outcomes or timepoints within a study. In: Borenstein M, Hedges LV, Higgins JPT, Rothstein HR, editors. Introduction to Meta-Analysis. Chichester, England: John Wiley; pp. $225-238$.

Borenstein, M., Hedges, L., Higgins, J. \& Rothstein, H. (2014) Comprehensive MetaAnalysis Version 2. Biostat, Englewood, New Jersey.

Borman, W. C., \& Motowidlo, S. J. (1997). Task performance and contextual performance: The meaning for personnel selection research. Human Performance, 10(2), 99-109.

*Boström, M., Sluiter, J. K., Hagberg, M., \& Grimby-Ekman, A. (2016). Opportunities for recovery at work and excellent work ability-a cross-sectional population study among young workers. BMC Public Health, 16(1), 985-996.

Brady, G., Rineer, J. R., Cadiz, D. M., \& Truxillo, D. M. (2016). Maintaining work ability to support and retain older workers. In The Aging Workforce Handbook: 
WORK ABILITY, AGE DISCRIMINATION, PERFORMANCE

Individual, Organizational, and Societal Challenges (pp. 323-353). Emerald Group Publishing Limited.

*Bridger, R. S., \& Bennett, A. I. (2011). Age and BMI interact to determine work ability in seafarers. Occupational Medicine, 61(3), 157-162.

*Bridger, R. S., Dobson, K., \& Davison, H. (2015). Using the HSE stress indicator tool in a military context. Ergonomics, 59 1-12.

*Bugajska, J., \& Sagan, A. (2014). Chronic musculoskeletal disorders as risk factors for reduced work ability in younger and ageing workers. International Journal of Occupational Safety and Ergonomics, 20, 607-615.

Butrica, B. (2011). Making a case for working longer at older ages. Public Policy \& Aging Report, 21, 20-24.

Cable, D. M., \& DeRue, D. S. (2002). The convergent and discriminant validity of subjective fit perceptions. Journal of Applied Psychology, 87(5), 875-884.

*Camerino, D., Conway, P. M., Estryn-Béhar, M., Costa, G., \& Hasselhorn, H. M. (2008). Age-dependent relationships between work ability, thinking of quitting the job, and actual leaving among Italian nurses: a longitudinal study. International Journal of Nursing Studies, 45(11), 1645-1659.

*Camerino, D., Conway, P. M., Van der Heijden, B. I. J., Estryn-Behar, M., Consonni, D., Gould, D., \& Hasselhorn, H. M. (2006). Low-perceived work ability, ageing and intention to leave nursing: a comparison among 10 European countries. Journal of Advanced Nursing, 56, 542-552. 
*Camerino, D., Conway, P. M., Sartori, S., Campanini, P., Estryn., Béhar, M., van der Heijden, B. I. J. M., \& Costa, G. (2008). Factors affecting work ability in day and shift working nurses. Chronobiology International, 25, 425-442.

Campbell, J. P., McCloy, R. A., Oppler, S. H., \& Sager, C. E. (1993). A theory of performance. Personnel selection in organizations, 3570, 35-70.

Campbell, J. P., \& Wiernik, B. M. (2015). The modeling and assessment of work performance. Annual. Review of Organizational Psychology and Organizational Behavior, 2(1), 47-74.

*Carel, R. S., Zusman, M., \& Karakis, I. (2013). Work ability index in Israeli hospital nurses: applicability of the adapted questionnaire. Experimental Aging Research, 39, 579-590.

*Chang, Y., ChenSea, M., Jang, Y., Wang, J. D. (2000). A simple self-rating assessment method of residual work capability for occupational permanent disabilities. American Journal of Industrial Medicine, 38, 539-547.

Chiu, W., Chan, A. W., Snape, E., \& Redman, T. (2001). Age stereotypes and discriminatory attitudes towards older workers: An East-West comparison. Human Relations, 54(5), 629-661.

*Chiu, M. C., Wang, M. J. J., Lu, C. W., Pan, S. M., Kumashiro, M., \& Ilmarinen, J. (2007). Evaluating work ability and quality of life for clinical nurses in Taiwan. Nursing Outlook, 55, 318-326. 
Christian, M. S., Garza, A. S., \& Slaughter, J. E. (2011). Work engagement: A quantitative review and test of its relations with task and contextual performance. Personnel Psychology, 64(1), 89-136.

*Chung, J., Park, J., Cho, M., Park, Y., Kim, D., Yang, D., \& Yang, Y. (2015). A study on the relationships between age, work experience, cognition, and work ability in older employees working in heavy industry. Journal of Physical Therapy Science, 27(1), 155-157.

*Claudi, J. A. (2012). Towards a parsimonious program theory of return to work intervention. Work, 44, 155-164.

Colquitt, J. A., Conlon, D. E., Wesson, M. J., Porter, C. O., \& Ng, K. Y. (2001). Justice at the millennium: a meta-analytic review of 25 years of organizational justice research. Journal of Applied Psychology, 86(3), 425-445.

Colquitt, J. A., Scott, B. A., Rodell, J. B., Long, D. M., Zapata, C. P., Conlon, D. E., \& Wesson, M. J. (2013). Justice at the millennium, a decade later: a meta-analytic test of social exchange and affect-based perspectives. Journal of Applied Psychology, 98(2), 199-236.

*Conway, P. M., Campanini, P., Sartori, S., Dotti, R., \& Costa, G. (2008). Main and interactive effects of shiftwork, age and work stress on health in an Italian sample of healthcare workers. Applied Ergonomics, 39, 630-639.

Cornelius III, E. T., Carron, T. J., \& Collins, M. N. (1979). Job analysis models and job classification. Personnel Psychology, 32(4), 693-708. 
Cortina, L. M., Kabat-Farr, D., Leskinen, E. A., Huerta, M., \& Magley, V. J. (2013). Selective incivility as modern discrimination in organizations: Evidence and impact. Journal of Management, 39(6), 1579-1605.

*Costa, A. F., Puga-Leal, R., \& Nunes, I. L. (2011). An exploratory study of the Work Ability Index (WAI) and its components in a group of computer workers. Work, 39(4), 357-367.

*Costa, G., \& Sartori, S. (2007). Ageing, working hours and work ability. Ergonomics, 50(11), 1914-1930.

Coverman, S. (1989). Role overload, role conflict, and stress: Addressing consequences of multiple role demands. Social Forces, 67(4), 965-982.

Crawford, E. R., LePine, J. A., \& Rich, B. L. (2010). Linking job demands and resources to employee engagement and burnout: a theoretical extension and meta-analytic test. Journal of Applied Psychology, 95(5), 834-848.

Cuddy, A. J., Norton, M. I., \& Fiske, S. T. (2005). This old stereotype: The pervasiveness and persistence of the elderly stereotype. Journal of Social Issues, 61(2), 267-285.

*D'amato, A., \& Zijlstra, F. (2010). Toward a climate for work resumption: the nonmedical determinants of return to work. Journal of Occupational and Environmental Medicine, 52(1), 67-80.

*da Silva, F. J., Felli, V. E. A., Martinez, M. C., Mininel, V. A., \& Ratier, A. P. P. (2016). Association between work ability and fatigue in Brazilian nursing workers. Work, 53(1), 225-232. 
*de Boer, A. G. E. M., Bruinvels, D. J., Tytgat, K. M. A. J., Schoorlemmer, A., Klinkenbijl, J. H. G., \& Frings-Dresen, M. H. W. (2011). Employment status and work-related problems of gastrointestinal cancer patients at diagnosis: A crosssectional study. BMJ open, 1(2), 1-8.

*de Croon, E. M., Sluiter, J. K., Nijssen, T. F., Kammeijer, M., Dijkmans, B. A. C., Lankhorst, G. J., \& Frings-Dresen, M. H. W. (2005). Work ability of Dutch employees with rheumatoid arthritis. Scandinavian Journal of Rheumatology, 34, 277-283.

*de Souza Magnago, T. S. B., de Lima, A. C. S., Prochnow, A., da Silva Ceron, M. D., Tavares, J. P., \& de Souza Urbanetto, J. (2012). Intensity of musculoskeletal pain and (in) ability to work in nursing. Revista Latino-American Enfermagem, 20, 1125-33.

*de Vries, H. J., Reneman, M. F., Groothoff, J. W., Geertzen, J. H., \& Brouwer, S. (2013). Self-reported work ability and work performance in workers with chronic nonspecific musculoskeletal pain. Journal of Occupational Rehabilitation, 23, 110.

Demerouti, E., \& Bakker, A. B. (2011). The job demands-resources model: Challenges for future research. Journal of Industrial Psychology, 37, 1-9.

Demerouti, E., Bakker, A. B., Nachreiner, F., \& Schaufeli, W. B. (2001). The job demands-resources model of burnout. Journal of Applied Psychology, 86, 499512. 
Dierdorff, E. C., \& Morgeson, F. P. (2007). Consensus in work role requirements: the influence of discrete occupational context on role expectations. Journal of Applied Psychology, 92(5), 1228.

Docquier, F., \& Rapoport, H. (2012). Globalization, brain drain, and development. Journal of Economic Literature, 50(3), 681-730.

Donaldson, S. I., \& Grant-Vallone, E. J. (2002). Understanding self-report bias in organizational behavior research. Journal of Business and Psychology, 17(2), $245-260$.

*Dukes, J. L. (2013). Health related quality of life and work ability in kidney transplant recipients (Doctoral dissertation, Saint Louis University).

Eatough, E. M., Chang, C. H., Miloslavic, S. A., \& Johnson, R. E. (2011). Relationships of role stressors with organizational citizenship behavior: A metaanalysis. Journal of Applied Psychology, 96(3), 619-632.

*Edlund, M., Gerhardsson, L., \& Hagberg, M. (2012). Physical capacity and psychological mood in association with self-reported work ability in vibrationexposed patients with hand symptoms. Journal of Occupational Medicine and Toxicology, 7, 1-7.

Edwards, J. R., (1991). Person-job fit: A conceptual integration, literature review, and methodological critique. In Cooper (Ed.) International Review of Industrial and Organizational Psychology 6, 283-357. Chichester, UK: Wiley. 
*Ekberg, K., Wåhlin, C., Persson, J., Bernfort, L., \& Öberg, B. (2011). Is mobility in the labor market a solution to sustainable return to work for some sick listed persons?. Journal of Occupational Rehabilitation, 21(3), 355.

*El Fassi, M., Bocquet, V., Majery, N., Lair, M. L., Couffignal, S., \& Mairiaux, P. (2013). Work ability assessment in a worker population: comparison and determinants of Work Ability Index and work ability score. BMC Public Health, $13,305-315$.

Eley, R., Hegney, D., Buikstra, E., Fallon, T., Plank, A., \& Parker, V. (2007). Aged care nursing in Queensland-the nurses' view. Journal of Clinical Nursing, 16(5), 860872.

*Elo, A. L., Ervasti, J., Kuosma, E., \& Mattila, P. (2008). Evaluation of an organizational stress management program in a municipal public works organization. Journal of Occupational Health Psychology, 13, 10-23.

*Elovainio, M., Kuusio, H., Aalto, A. M., Sinervo, T., \& Heponiemi, T. (2010). Insecurity and shiftwork as characteristics of negative work environment: psychosocial and behavioural mediators. Journal of Advanced Nursing, 66, 10801091.

*Emmerich, A. I., \& Rigotti, T. (2017). Reciprocal relations between work-related authenticity and intrinsic motivation, work ability and depressivity: A two-wave study. Frontiers in Psychology, 8, 1-12. 
Enders, C. K., \& Bandalos, D. L. (2001). The relative performance of full information maximum likelihood estimation for missing data in structural equation models. Structural Equation Modeling, 8(3), 430-457.

Faragher, E. B., Cass, M., \& Cooper, C. L. (2005). The relationship between job satisfaction and health: a meta-analysis. Occupational and Environmental Medicine, 62, 105-112.

Feldman, D. C. (1994). The decision to retire early: A review and conceptualization. Academy of Management Review, 19, 285-311.

*Feldt, T., Hyvönen, K., Mäkikangas, A., Kinnunen, U., \& Kokko, K. (2009). Development trajectories of Finnish managers' work ability over a 10-year follow-up period. Scandinavian Journal of Work, Environment \& Health, 35, 3747.

*Feldt, T., Hyvönen, K., Oja-Lipasti, T., Kinnunen, U., \& Salmela-Aro, K. (2012). Do work ability and job involvement channel later personal goals in retirement? An 11-year follow-up study. International Archives of Occupational and Environmental Health, 85, 547-558.

*Firoozeh, M., Saremi, M., Kavousi, A., \& Maleki, A. (2017). Demographic and occupational determinants of the work ability of firemen. Journal of Occupational Health, 59(1), 81-87.

*Fischer, F. M., Borges, F. D. S., Rotenberg, L., Latorre, M. R. D. O., Soares, N. S., Rosa, P. F. L. S., \& Landsbergis, P. (2006). Work ability of health care shift workers: what matters?. Chronobiology International, 23, 1,165-1,179. 
*Fischer, F. M., \& Martinez, M. C. (2011). Work ability among hospital food service professionals: multiple associated variables require comprehensive intervention. Work, 41, 3,746-3,752.

*Fischer, F. M., \& Martinez, M. C. (2012). Individual features, working conditions and work injuries are associated with work ability among nursing professionals. Work, 45, 509-517.

Fisher, G. G., Truxillo, D. M., Finkelstein, L. M., \& Wallace, L. E. (2017). Age discrimination: Potential for adverse impact and differential prediction related to age. Human Resource Management Review, 27(2), 316-327.

Fiske, S. T., Cuddy, A. J., Glick, P., \& Xu, J. (2002). A model of (often mixed) stereotype content: competence and warmth respectively follow from perceived status and competition. Journal of Personality and Social Psychology, 82(6), 878-

*Furu, H., Sainio, M., Ahonen, G., Hyvärinen, H. K., \& Kaukiainen, A. (2014). Cost of detecting a chronic solvent encephalopathy case by screening. Neurotoxicology, 45, 253-259.

Furunes, T., \& Mykletun, R. J. (2010). Age discrimination in the workplace: Validation of the Nordic Age Discrimination Scale (NADS). Scandinavian Journal of Psychology, 51(1), 23-30.

*Gartoulla, P., Bell, R. J., Worsley, R., \& Davis, S. R. (2016). Menopausal vasomotor symptoms are associated with poor self-assessed work ability. Maturitas, 87, 3339. 
*Geukes, M., van Aalst, M. P., Nauta, M. C., \& Oosterhof, H. (2012). The impact of menopausal symptoms on work ability. Menopause, 19, 278-282.

*Ghaddar, A., Ronda, E., \& Nolasco, A. (2011). Work ability, psychosocial hazards and work experience in prison environments. Occupational Medicine, 61(7), 503-508.

Gilliland, S. W. (1993). The perceived fairness of selection systems: An organizational justice perspective. Academy of Management Review, 18(4), 694-734.

*Glise, K., Hadzibajramovic, E., Jonsdottir, I. H., \& Ahlborg, G. (2010). Self-reported exhaustion: a possible indicator of reduced work ability and increased risk of sickness absence among human service workers. International Archives of Occupational and Environmental Health, 83(5), 511-520.

*Godinho, M. R., Greco, R. M., Teixeira, M. T. B., Teixeira, L. R., Guerra, M. R., \& Chaoubah, A. (2016). Work ability and associated factors of Brazilian technicaladministrative workers in education. BMC Research Notes, 9(1), 1-10.

Goldberg, L. R. (1990). An alternative" description of personality": the big-five factor structure. Journal of Personality and Social Psychology, 59(6), 1216-1229.

*Golubic, R., Milosevic, M., Knezevic, B., \& Mustajbegovic, J. (2009). Work related stress, education and work ability among hospital nurses. Journal of Advanced Nursing, 65, 2056-2066.

*Graafmans, J. (1998). Age, absenteeism and physical fitness in relation to work ability. Gerontechnology: A Sustainable Investment in the Future, 48, 254-265. 
Griffin, M. A., Neal, A., \& Parker, S. K. (2007). A new model of work role performance: Positive behavior in uncertain and interdependent contexts. Academy of Management Journal, 50(2), 327-347.

*Gudbergsson, S. B., Fosså, S. D., \& Dahl, A. A. (2008). Is cancer survivorship associated with reduced work engagement? A NOCWO Study. Journal of Cancer Survivorship, 2(3), 159-168.

*Guidi, S., Bagnara, S., \& Fichera, G. P. (2012). The HSE indicator tool, psychological distress and work ability. Occupational Medicine, 62, 203-209.

*Gupta, N., Jensen, B. S., Søgaard, K., Carneiro, I. G., Christiansen, C. S., Hanisch, C., \& Holtermann, A. (2014). Face validity of the single work ability item: comparison with objectively measured heart rate reserve over several days. International Journal of Environmental Research and Public Health, 11, 5,3335,348 .

*Gustafsson, K., \& Marklund, S. (2011). Consequences of sickness presence and sickness absence on health and work ability: a Swedish prospective cohort study. International Journal of Occupational Medicine and Environmental Health, 24, 153-165.

*Habibi, E., Dehghan, H., Safari, S., Mahaki, B., \& Hassanzadeh, A. (2014). Effects of work-related stress on work ability index among refinery workers. Journal of Education and Health Promotion, 3, 43-48.

*Habibi, E., Dehghan, H., Zeinodini, M., Yousefi, H., \& Hasanzadeh, A. (2012). A study on work ability index and physical work capacity on the base of fax equation VO2 
max in male nursing hospital staff in Isfahan, Iran. International Journal of Preventive Medicine, 3(11), 776-782.

*Hakanen, J. J., Bakker, A. B., \& Schaufeli, W. B. (2006). Burnout and work engagement among teachers. Journal of School Psychology, 43, 495-513.

*Hakkarainen, P., Moilanen, L., Hänninen, V., Heikkinen, J., \& Räsänen, K. (2016). Work ability among Finnish workers with type 1 diabetes. Occupational Medicine, 66(6), 446-453.

*Han, L., Shi, L., Lu, L., \& Ling, L. (2014). Work ability of Chinese migrant workers: the influence of migration characteristics. BMC Public Health, 14, 1-8.

*Hansen, K. E., Kesmodel, U. S., Baldursson, E. B., Schultz, R., \& Forman, A. (2013). The influence of endometriosis-related symptoms on work life and work ability: a study of Danish endometriosis patients in employment. European Journal of Obstetrics \& Gynecology and Reproductive Biology, 169, 331-339.

Hammond, M. M., Neff, N. L., Farr, J. L., Schwall, A. R., \& Zhao, X. (2011). Predictors of individual-level innovation at work: A meta-analysis. Psychology of Aesthetics, Creativity, and the Arts, 5(1), 90-105.

Heckhausen, J., Wrosch, C., \& Schulz, R. (2010). A motivational theory of life-span development. Psychological Review, 117(1), 32-82.

*Hedenrud, T., Löve, J., Staland-Nyman, C., \& Hensing, G. (2014). Frequent headache and work ability: a population-based study in Sweden. Journal of Occupational and Environmental Medicine, 56(5), 472-476. 
Hedges, L. V., \& Olkin, I. (1985). Statistical methods for meta-analysis. Orlando, FL: Academic Press.

Hedges, L. V., \& Pigott, T. D. (2001). The power of statistical tests in metaanalysis. Psychological Methods, 6(3), 203-217.

*Hellemans, C., \& Lapthorn, B. (2016). Antecedents of work ability in the cleaning sector: From health, competence and pleasure at work to working conditions. International Journal of Workplace Health Management, 9(3), 328339.

*Heponiemi, T., Kouvonen, A., Vänskä, J., Halila, H., Sinervo, T., Kivimäki, M., \& Elovainio, M. (2008). Health, psychosocial factors and retirement intentions among Finnish physicians. Occupational Medicine, 58, 406-412.

Herzog, A., House, J. S., \& Morgan, J. N. (1991). Relation of work and retirement to health and well-being in older age. Psychology and Aging, 6, 202-211.

Higgins, J. P. T., \& Thompson, S. G. (2002). Quantifying heterogeneity in a metaanalysis. Statistics in Medicine, 21, 1539-1558. doi:10.1002/sim.1186

Hinkin, T. R. (1995). A review of scale development practices in the study of organizations. Journal of Management, 21(5), 967-988.

Hobfoll, S. E. (1989). Conservation of resources: A new attempt at conceptualizing stress. American Psychologist, 44(3), 513-524.

*Holm, H. V., Fosså, S. D., Hedlund, H., \& Dahl, A. A. (2013). Study of generic quality of life in patients operated on for post-prostatectomy incontinence. International Journal of Urology, 20(9), 889-895. 
*Holtermann, A., Hansen, J. V., Burr, H., \& Søgaard, K. (2010). Prognostic factors for long-term sickness absence among employees with neck-shoulder and low-back pain. Scandinavian Journal of Work, Environment \& Health, 36, 34-41.

*Hoonakker, P., \& Van Duivenbooden, C. (2012). Predicting long-term absenteeism from work in construction industry: a longitudinal study. Work, 41(1), 3765-3770.

Huedo-Medina, T. B., Sánchez-Meca, J., Marín-Martínez, F., \& Botella, J. (2006). Assessing heterogeneity in meta-analysis: Q statistic or $\mathrm{I}^{2}$ index? Psychological Methods, 11, 193-206.

Humphrey, S. E., Nahrgang, J. D., \& Morgeson, F. P. (2007). Integrating motivational, social, and contextual work design features: a meta-analytic summary and theoretical extension of the work design literature. Journal of Applied Psychology, 92(5), 1332-1356.

*Ilmarinen, J., \& Tuomi, K. (1992). Work ability of aging workers. Scandinavian Journal of Work, Environment \& Health, 8-10.

Ilmarinen, J. (2009). Work ability — a comprehensive concept for occupational health research and prevention. Scandinavian Journal of Work, Environment \& Health, $35,1-5$.

*Ilmarinen, V., Ilmarinen, J., Huuhtanen, P., Louhevaara, V., \& Näsman, O. (2015). Examining the factorial structure, measurement invariance and convergent and discriminant validity of a novel self-report measure of work ability: work abilitypersonal radar. Ergonomics, 58, 1445-1460. 
Ilmarinen, J., Tuomi, K., Eskelinen, L., Nygård, C. H., Huuhtanen, P., \& Klockars, M. (1991a). Background and objectives of the Finnish research project on aging workers in municipal occupations. Scandinavian Journal of Work, Environment \& Health, 17, 7-11.

*Ilmarinen, J., Tuomi, K., Eskelinen, L., Nygård, C. H., Huuhtanen, P., \& Klockars, M. (1991b). Summary and recommendations of a project involving cross-sectional and follow-up studies on the aging worker in Finnish municipal occupations IlmI(1981-1985). Scandinavian Journal of Work, Environment \& Health, 17, $135-141$.

Ilmarinen, J., Tuomi, K., \& Klockars, M. (1997). Changes in the work ability of active employees over an 11-year period. Scandinavian Journal of Work, Environment \& Health, 23, 49-57.

Janssen, O., \& Van Yperen, N. W. (2004). Employees' goal orientations, the quality of leader-member exchange, and the outcomes of job performance and job satisfaction. Academy of Management Journal, 47(3), 368-384.

*Ję dryka-Góral, A., Bugajska, J., Łastowiecka, E., Najmiec, A., Rell-Bakalarska, M., Bownik, I., \& Kochmański, M. (2006). Work ability in ageing workers suffering from chronic diseases. International Journal of Occupational Safety and Ergonomics, 12, 17-30.

Judge, T. A., \& Bono, J. E. (2001). Relationship of core self-evaluations traits—-selfesteem, generalized self-efficacy, locus of control, and emotional stability — with 
job satisfaction and job performance: A meta-analysis. Journal of Applied Psychology, 86, 80-92.

Judge, T. A., Heller, D., \& Mount, M. K. (2002). Five-factor model of personality and job satisfaction: A meta-analysis. Journal of Applied Psychology, 87(3), 530-541.

Judge, T. A., \& Kammeyer-Mueller, J. D. (2012). Job attitudes. Annual Review of Psychology, 63, 341-367.

Judge, T. A., \& Piccolo, R. F. (2004). Transformational and transactional leadership: a meta-analytic test of their relative validity. Journal of Applied Psychology, 89(5), $755-768$.

*Kabat-Farr, D., Walsh, B. M., \& McGonagle, A. K. Uncivil supervisors and perceived work ability: The joint moderating roles of job involvement and grit. Journal of Business Ethics, 1-15.

*Kaewboonchoo, O., \& Ratanasiripong, P. (2015). Psychometric properties of the Thai version of the work ability index (Thai WAI). Journal of Occupational Health, $57,371-377$.

*Kaewboonchoo, O., Isahak, M., Susilowati, I., Phuong, T. N., Morioka, I., Harncharoen, K., \& Ratanasiripong, P. (2016). Work ability and its related factors among workers in small and medium enterprises: Comparison among four ASEAN countries. Asia Pacific Journal of Public Health, 28(5), 438-449.

Kahn, W. A. (1990). Psychological conditions of personal engagement and disengagement at work. Academy of Management Journal, 33(4), 692-724. 
*Kaleta, D., Makowiec-Dąbrowska, T., \& Jegier, A. N. N. A. (2004). Leisure-time physical activity, cardiorespiratory fitness and work ability: A study in randomly selected residents of Łódź. International Journal Occupational Medicine \& Environmental Health, 17(4), 457-64.

*Kaleta, D., Makowiec-Dąbrowska, T., \& Jegier, A. (2006). Lifestyle index and work ability. International Journal of Occupational Medicine and Environmental Health, 19, 170-177.

Karasek, R.A. (1979). Job demands, job decision latitude, and mental strain: Implications for job redesign. Administrative Science Quarterly, 24, 285-308. doi: $10.2307 / 2392498$.

Karasek, R. A., Triantis, K. P., \& Chaudhry, S. S. (1982). Coworker and supervisor support as moderators of associations between task characteristics and mental strain. Journal of Organizational Behavior, 3(2), 181-200.

*Karttunen, J. P., \& Rautiainen, R. H. (2009). Work ability index among Finnish dairy farmers. Journal of Agricultural Safety and Health, 15(4), 353-364.

*Karttunen, J. P., \& Rautiainen, R. H. (2011). Risk factors and prevalence of declined work ability among dairy farmers. Journal of Agricultural Safety and Health, 17, 243-257.

*Karvala, K., Nordman, H., Luukkonen, R., \& Uitti, J. (2014). Asthma related to workplace dampness and impaired work ability. International Archives of Occupational and Environmental Health, 87, 1-11. 
Kepes, S., McDaniel, M. A., Brannick, M. T., \& Banks, G. C. (2013). Meta-analytic reviews in the organizational sciences: Two meta-analytic schools on the way to MARS (the Meta-Analytic Reporting Standards). Journal of Business and Psychology, 28, 123-143.

*Kinnunen, U., Parkatti, T., \& Rasku, A. (1994). Occupational well-being among aging teachers in Finland. Scandinavian Journal of Educational Research, 38, 315-332.

*Kinnunen, U., Parkatti, T., \& Rasku, A. (1994). Occupational well-being among aging teachers in Finland. Scandinavian Journal of Educational Research, 38(3-4), 315332.

Kooij, D. T., Tims, M., \& Kanfer, R. (2015). Successful aging at work: The role of job crafting. In Aging Workers and the Employee-Employer Relationship (pp. 145161). New York: Springer.

*Koolhaas, W., van der Klink, J. J., de Boer, M. R., Groothoff, J. W., \& Brouwer, S. (2014). Chronic health conditions and work ability in the ageing workforce: the impact of work conditions, psychosocial factors and perceived health. International Archives of Occupational and Environmental Health, 87(4), 433-443.

Koopman, J., Howe, M., Hollenbeck, J. R., \& Sin, H. P. (2015). Small sample mediation testing: Misplaced confidence in bootstrapped confidence intervals. Journal of Applied Psychology, 100(1), 194-202.

Koopmann, J., Lanaj, K., Wang, M., Zhou, L., \& Shi, J. (2016). Nonlinear effects of team tenure on team psychological safety climate and climate strength: Implications for 
average team member performance. Journal of Applied Psychology, 101(7), 940957.

Kristof-Brown, A. L., Zimmerman, R. D., \& Johnson, E. C. (2005). Consequences of individuals' fit at work: A meta-analysis of person-job, person-organization, person-group, and person-supervisor fit. Personnel Psychology, 58(2), 281-342.

Kruger, J., \& Dunning, D. (1999). Unskilled and unaware of it: how difficulties in recognizing one's own incompetence lead to inflated self-assessments. Journal of personality and social psychology, 77(6), 1121-1134.

*Kujala, V., Tammelin, T., Remes, J., Vammavaara, E., Ek, E., \& Laitinen, J. (2006). Work ability index of young employees and their sickness absence during the following year. Scandinavian Journal of Work, Environment \& Health, 75-84.

*Kuoppala, J., Lamminpää, A., Väänänen-Tomppo, I., \& Hinkka, K. (2011). Employee well-being and sick leave, occupational accident, and disability pension: A cohort study of civil servants. Journal of Occupational and Environmental Medicine, 53(6), 633-640.

Kulik, C. T., Ryan, S., Harper, S., \& George, G. (2014). Aging populations and management. Academy of Management Journal, 57, 929-935.

*Kuusio, H., Heponiemi, T., Aalto, A. M., Sinervo, T., \& Elovainio, M. (2012). Differences in well-being between GPs, medical specialists, and private physicians: The role of psychosocial factors. Health Services Research, 47, 68-85.

*Labbafinejad, Y., Ghaffari, M., Bahadori, B., Mohammadi, S., Abdi, A., Namvar, M., \& Attarchi, M. (2014). The effect of sleep disorder on the work ability of workers in 
a car accessories manufacturing plant. Medical Journal of the Islamic Republic of Iran, 28, 111-119.

*Laitinen, J., Näyhä, S., \& Kujala, V. (2005). Body mass index and weight change from adolescence into adulthood, waist-to-hip ratio and perceived work ability among young adults. International Journal of Obesity, 29, 697-702.

Lamont, R. A., Swift, H. J., \& Abrams, D. (2015). A review and meta-analysis of agebased stereotype threat: Negative stereotypes, not facts, do the damage. Psychology and Aging, 30(1), 180-193.

*Larsson, A., Karlqvist, L., Westerberg, M., \& Gard, G. (2012). Identifying work ability promoting factors for home care aides and assistant nurses. BMC Musculoskeletal Disorders, 13, 1-11.

*Lastowiecka, E., Bugajska, J., Najmiec, A., Rell-Bakalarska, M., Bownik, I., \& JędrykaGóral, A. (2006). Occupational work and quality of life in osteoarthritis patients. Rheumatology International, 27(2), 131-139.

*Leggett, S., Van Der Zee-Neuen, A., Boonen, A., Beaton, D. E., Bojinca, M., Bosworth, A., \& Lacaille, D. (2016). Test-retest reliability and correlations of 5 global measures addressing at-work productivity loss in patients with rheumatic diseases. The Journal of Rheumatology, 43(2), 433-439.

*Leijten, F. R., van den Heuvel, S. G., Ybema, J. F., van der Beek, A. J., Robroek, S. J., \& Burdorf, A. (2014). The influence of chronic health problems on work ability and productivity at work: A longitudinal study among older employees. Scandinavian Journal of Work, Environment \& Health, 473-482. 
Levine, E. L., Ash, R. A., Hall, H., \& Sistrunk, F. (1983). Evaluation of job analysis methods by experienced job analysts. Academy of Management Journal, 26(2), 339-348.

Levy, B. R., \& Banaji, M. R. (2002). Implicit ageism. Ageism: Stereotyping and Prejudice Against Older Persons, 49-75.

*Li, H., Liu, Z., Liu, R., Li, L., \& Lin, A. (2015). The relationship between work stress and work ability among power supply workers in Guangdong, China: A crosssectional study. BMC Public Health, 16(1), 123.

*Lian, Y., Xiao, J., Liu, Y., Ning, L., Guan, S., Ge, H., \& Liu, J. (2015). Associations between insomnia, sleep duration and poor work ability. Journal of Psychosomatic Research, 78(1), 45-51.

*Lin, S., Wang, Z., \& Wang, M. (2006). Work ability of workers in western China: reference data. Occupational Medicine, 56, 89-93.

*Lindbohm, M. L., Taskila, T., Kuosma, E., Hietanen, P., Carlsen, K., Gudbergsson, S., \& Gunnarsdottir, H. (2012). Work ability of survivors of breast, prostate, and testicular cancer in Nordic countries: a NOCWO study. Journal of Cancer Survivorship, 6(1), 72-81.

*Lindegård, A., Larsman, P., Hadzibajramovic, E., \& Ahlborg, G. (2014). The influence of perceived stress and musculoskeletal pain on work performance and work ability in Swedish health care workers. International Archives of Occupational and Environmental Health, 87(4), 373-379. 
*Lindström, I., Pallasaho, P., Luukkonen, R., Suojalehto, H., Karjalainen, J., Lauerma, A., \& Karjalainen, A. (2011). Reduced work ability in middle-aged men with asthma from youth-a 20-year follow-up. Respiratory Medicine, 105(6), 950-955.

Little, R. J., \& Rubin, D. B. (1987). Statistical analysis with missing data. New York: Wiley \& Sons.

Lord, R. L., \& Farrington, P. A. (2006). Age-related differences in the motivation of knowledge workers. Engineering Management Journal, 18(3), 20-26.

*Löve, J., Holmgren, K., Torén, K., \& Hensing, G. (2012). Can work ability explain the social gradient in sickness absence: a study of a general population in Sweden. BMC Public Health, 12(1), 163-172.

*Lundin, A., Leijon, O., Vaez, M., Hallgren, M., \& Torgén, M. (2017). Predictive validity of the Work Ability Index and its individual items in the general population. Scandinavian Journal of Public Health, 45(4), 350-356.

*Lundin, A., Kjellberg, K., Leijon, O., Punnett, L., \& Hemmingsson, T. (2016). The association between self-assessed future work ability and long-term sickness absence, disability pension and unemployment in a general working population: a 7-year follow-up study. Journal of Occupational Rehabilitation, 26(2), 195-203.

*Mache, S., Danzer, G., Klapp, B. F., \& Groneberg, D. A. (2013). Surgeons' work ability and performance in surgical care: relations between organisational predictors, work engagement and work ability. Langenbeck's Archives of Surgery, 398, 317325. 
*Madeleine, P., Vangsgaard, S., Andersen, J. H., Ge, H. Y., \& Arendt-Nielsen, L. (2013). Computer work and self-reported variables on anthropometrics, computer usage, work ability, productivity, pain, and physical activity. BMC Musculoskeletal Disorders, 14, 1-12.

*Magnago, T., Lima, A., Prochnow, A., Ceron, M., Tavares, J. P., \& Urbanetto, J. D. S. (2012). Intensity of musculoskeletal pain and (in) ability to work in nursing. Revista Latino-Americana de Enfermagem, 20, 1125-1133.

Manchikanti, L., Singh, V., Datta, S., Cohen, S. P., \& Hirsch, J. A. (2008). Comprehensive review of epidemiology, scope, and impact of spinal pain. Pain Physician, 12, 35-70.

Marchiondo, L. A., Gonzales, E., \& Ran, S. Development and validation of the workplace age discrimination scale. Journal of Business and Psychology, 1-21.

*Marklund, S., Bolin, M., \& von Essen, J. (2008). Can individual health differences be explained by workplace characteristics?-A multilevel analysis. Social Science \& Medicine, 66(3), 650-662.

*Marqueze, E. C., Voltz, G. P., Borges, F. N., \& Moreno, C. R. (2008). A 2-year followup study of work ability among college educators. Applied Ergonomics, 39, 640645.

*Martinez, M. C., Latorre, M. D. R. D. D., \& Fischer, F. M. (2009). Validity and reliability of the Brazilian version of the Work Ability Index questionnaire. Revista de Saude Publica, 43, 525-532. 
Martus, P., Jakob, O., Rose, U., Seibt, R., \& Freude, G. (2010). A comparative analysis of the Work Ability Index. Occupational Medicine, 60(7), 517-524.

Maslowsky, J., Jager, J., \& Hemken, D. (2015). Estimating and interpreting latent variable interactions: A tutorial for applying the latent moderated structural equations method. International Journal of Behavioral Development, 39(1), 8796.

*McGonagle, A. K., \& Barnes-Farrell, J. L. (2014). Chronic illness in the workplace: Stigma, identity threat and strain. Stress and Health, 30, 310-321.

*McGonagle, A.K., Barnes-Farrell, J.L., Di Milia, L., Fischer, F.M., Hobbs, B., IskraGolec, I., Kaliterna, L., \& Smith, L. (2014). Demands, resources, and work ability: A cross-national examination of health care workers. European Journal of Work and Organizational Psychology, 23, 830-846.

*McGonagle, A.K., Fisher, G.G., Barnes-Farrell, J.L., and Grosch, J.W. (2015). Individual and work factors related to perceived work ability and labor force outcomes. Journal of Applied Psychology, 100, 376-398.

*Mehrdad, R., Mazloumi, A., Arshi, S., \& Kazemi, Z. (2016). Work Ability Index among healthcare personnel in a university hospital in Tehran, Iran. Work, 53(4), 851857.

*Milani, D., \& Monteiro, M. S. (2012). Musculoskeletal symptoms and work ability among agricultural machinery operators. Work, 41, 5,721-5,724. 
Millanvoye, M. (1998). Ageing of the organism before sixty years of age. In: J.C., Marquie, D. Paumes Cau-Bareille, \& S. Volkoff, (Eds.) Working with Age (pp. 133-161). London: Taylor \& Francis Inc.

*Milosevic, M., Golubic, R., Knezevic, B., Golubic, K., Bubas, M., \& Mustajbegovic, J. (2011). Work ability as a major determinant of clinical nurses' quality of life. Journal of Clinical Nursing, 20, 2931-2938.

Moghimi, D., Zacher, H., Scheibe, S., \& Van Yperen, N. W. (2017). The selection, optimization, and compensation model in the work context: A systematic review and meta-analysis of two decades of research. Journal of Organizational Behavior, 38(2), 247-275.

*Mohammadi, S., Ghaffari, M., Abdi, A., Bahadori, B., Mirzamohammadi, E., \& Attarchi, M. (2015). Interaction of Lifestyle and Work Ability Index in Blue Collar Workers. Global Journal of Health Science, 7, 90-97.

*Möller, L. M., Brands, R., Sluiter, J. K., Schouten, J., Wit, F. W., Reiss, P., \& Stolte, I. G. (2016). Prevalence and determinants of insufficient work ability in older HIVpositive and HIV-negative workers. International Archives of Occupational and Environmental Health, 89(4), 699-709.

*Momsen, A. M. H., Jensen, O. K., Nielsen, C. V., \& Jensen, C. (2014). Multiple somatic symptoms in employees participating in a randomized controlled trial associated with sickness absence because of nonspecific low back pain. The Spine Journal, 14(12), 2868-2876. 
*Monteiro, M. S., Ilmarinen, J., \& Filho, H. R. C. (2006). Work ability of workers in different age groups in a public health institution in Brazil. International Journal of Occupational Safety and Ergonomics, 12(4), 417-427.

*Monteiro, M. S., \& Alexandre, N. M. C. (2009). Work ability and low back pain among workers from a public health institution. Revista Gaúcha de Enfermagem, 30, 297-302.

Morgeson, F. P., \& Dierdorff, E. C. (2011). Work analysis: From technique to theory. APA Handbook of Industrial and Organizational Psychology, 2, 3-41.

Morgeson, F. P., \& Humphrey, S. E. (2006). The Work Design Questionnaire (WDQ): developing and validating a comprehensive measure for assessing job design and the nature of work. Journal of Applied Psychology, 91(6), 1321-1339.

Morschhäuser, M., \& Sochert, R. (2006). Healthy work in an ageing Europe. Federal Association of Company Health Insurance Funds, Essen, Germany, 1-76.

Motowidlo, S. J., \& Van Scotter, J. R. (1994). Evidence that task performance should be distinguished from contextual performance. Journal of Applied Psychology, 79(4), 475-480.

*Müller, A., Heiden, B., Herbig, B., Poppe, F., \& Angerer, P. (2016). Improving wellbeing at work: A randomized controlled intervention based on selection, optimization, and compensation. Journal of Occupational Health Psychology, 21, 169-181. 
*Müller, A., Weigl, M., Heiden, B., Glaser, J., \& Angerer, P. (2012). Promoting work ability and well-being in hospital nursing: The interplay of age, job control, and successful ageing strategies. Work, 41, 5137-5144.

*Müller, A., Weigl, M., Heiden, B., Herbig, B., Glaser, J., \& Angerer, P. (2013).

Selection, optimization, and compensation in nursing: exploration of job-specific strategies, scale development, and age-specific associations to work ability. Journal of Advanced Nursing, 69, 1630-1642.

*Neri, L., Rocca, R. L., Gallieni, M., Brancaccio, D., Cozzolino, M., Colombi, A., \& Burroughs, T. E. (2009). Occupational stress is associated with impaired work ability and reduced quality of life in patients with chronic kidney failure. The International Journal of Artificial Organs, 32, 291-298.

*Netterstrøm, B., Eller, N. H., \& Borritz, M. (2015). Prognostic factors of returning to work after sick leave due to work-related common mental disorders: a one-and three-year follow-up study. BioMed Research International, 2015.

*Neupane, S., Virtanen, P., Leino-Arjas, P., Miranda, H., Siukola, A., \& Nygård, C. H. (2013). Multi-site pain and working conditions as predictors of work ability in a 4-year follow-up among food industry employees. European Journal of Pain, 17, 444-451.

*Neupane, S., Virtanen, P., Luukkaala, T., Siukola, A., \& Nygård, C. H. (2014). A fouryear follow-up study of physical working conditions and perceived mental and physical strain among food industry workers. Applied Ergonomics, 45(3), 586591. 
*Nevanperä, N., Seitsamo, J., Ala-Mursula, L., Remes, J., Hopsu, L., Auvinen, J., \& Laitinen, J. (2016). Perceived work ability in the light of long-term and stressrelated unhealthy behaviors - a prospective cohort study. International Journal of Behavioral Medicine, 23(2), 179-189.

Ng, T. W., \& Feldman, D. C. (2008). The relationship of age to ten dimensions of job performance. Journal of Applied Psychology, 93(2), 392-423.

Ng, T. W., \& Feldman, D. C. (2012). Evaluating six common stereotypes about older workers with meta-analytical data. Personnel Psychology, 65(4), 821-858.

Ng, T. W., \& Feldman, D. C. (2013). A meta-analysis of the relationships of age and tenure with innovation-related behavior. Journal of Occupational and Organizational Psychology, 86(4), 585-616.

*Niessen, M. A., Laan, E. L., Robroek, S. J., Essink-Bot, M. L., Peek, N., Kraaijenhagen, R. A., \& Burdorf, A. (2013). Determinants of participation in a web-based health risk assessment and consequences for health promotion programs. Journal of Medical Internet Research, 15, 1-16.

*Nilsson, S., \& Ekberg, K. (2013). Employability and work ability: Returning to the labour market after long-term absence. Work: A Journal of Prevention, Assesment and Rehabilitation, 44, 449-457.

Ng, T. W., \& Feldman, D. C. (2008). The relationship of age to ten dimensions of job performance. Journal of Applied Psychology, 93, 392-423.

Noone, J. H., Mackey, M.G., \& Bohle, P. (2014). Work ability in Australia - pilot study: A report to Safe Work Australia. Canberra: Safe Work Australia. 
*Nygård, C. H., Eskelinen, L., Suvanto, S., Tuomi, K., \& Ilmarinen, J. (1991). Associations between functional capacity and work ability among elderly municipal employees. Scandinavian Journal of Work, Environment \& Health, $122-127$.

*Oakman, J., Neupane, S., \& Nygård, C. H. (2016). Does age matter in predicting musculoskeletal disorder risk? An analysis of workplace predictors over 4 years. International Archives of Occupational and Environmental Health, 89(7), 11271136.

*Oakman, J., \& Wells, Y. (2016). Working longer: What is the relationship between person-environment fit and retirement intentions?. Asia Pacific Journal of Human Resources, 54(2), 207-229.

*Oberlinner, C., Yong, M., Nasterlack, M., Pluto, R. P., \& Lang, S. (2015). Combined effect of back pain and stress on work ability. Occupational Medicine, 65(2), 147153.

*Ohta, M., Eguchi, Y., Inoue, T., Honda, T., Morita, Y., Konno, Y., \& Kumashiro, M. (2015). Effects of bench step exercise intervention on work ability in terms of cardiovascular risk factors and oxidative stress: a randomized controlled study. International Journal of Occupational Safety and Ergonomics, 21, 141-149.

*Ohta, M., Kumashiro, M., Eguchi, Y., Morita, Y., Konno, Y., \& Yamato, H. (2014). The relationship between work ability and oxidative stress in Japanese workers. Ergonomics, 57(8), 1265-1273. 
Oldham, G. R., \& Cummings, A. (1996). Employee creativity: Personal and contextual factors at work. Academy of Management Journal, 39(3), 607-634.

*Padula, R. S., Comper, M. L., Moraes, S. A., Sabbagh, C., Pagliato Junior, W., \& Perracini, M. R. (2013). The work ability index and functional capacity among older workers. Brazilian Journal of Physical Therapy, 17, 382-391.

*Palermo, J., Fuller-Tyszkiewicz, M., Walker, A., \& Appannah, A. (2013). Primary-and secondary-level organizational predictors of work ability. Journal of Occupational Health Psychology, 18, 220-229.

Pedersen, B. K., \& Saltin, B. (2006). Evidence for prescribing exercise as therapy in chronic disease. Scandinavian Journal of Medicine \& Science in Sports, 16, 3-63.

Penedo, F. J., \& Dahn, J. R. (2005). Exercise and well-being: a review of mental and physical health benefits associated with physical activity. Current Opinion in Psychiatry, 18, 189-193.

*Perkiö-Mäkelä, M. M. (2000). Finnish farmers' self-reported morbidity, work ability, and functional capacity. Annals of Agricultural and Environmental Medicine, 7(1), 11-16.

Perry, E. L., Dokko, G., \& Golom, F. D. (2012). The aging worker and personenvironment fit. The Oxford Handbook of Work and Aging, 187-212.

*Phongamwong C, Deema H. The impact of multi-site musculoskeletal pain on work ability among health care providers. (2015). Journal of Occupational Medicine and Toxicology, 1, 1-10. 
*Pilger, A., Haslacher, H., Ponocny-Seliger, E., Perkmann, T., Böhm, K., Budinsky, A., \& Winker, R. (2014). Affective and inflammatory responses among orchestra musicians in performance situation. Brain, Behavior, and Immunity, 37, 23-29.

*Pit, S. W., \& Hansen, V. (2014). Factors influencing early retirement intentions in Australian rural general practitioners. Occupational Medicine, 64, 297-304

*Plat, M. C. J., Frings-Dresen, M. H., \& Sluiter, J. K. (2010). Clinimetric quality of the fire fighting simulation test as part of the Dutch fire fighters Workers' Health Surveillance. BMC Health Services Research, 10(1), 32-41.

*Plat, M. C. J., Frings-Dresen, M. H., \& Sluiter, J. K. (2010). Reproducibility and validity of the stair-climb test for fire fighters. International Archives of Occupational and Environmental Health, 83(7), 725-731.

*Plat, M. C. J., Frings-Dresen, M. H., \& Sluiter, J. K. (2012). Impact of chronic diseases on work ability in ageing firefighters. Journal of Occupational Health, 54, 158163.

Podsakoff, P. M., \& MacKenzie, S. B. 1989. A second generation measure of organizational citizenship behavior. Unpublished manuscript, Indiana University, Bloomington

Podsakoff, P. M., MacKenzie, S. B., Lee, J., \& Podsakoff, N. P. (2003). Common method biases in behavioral research: A critical review of the literature and recommended remedies. Journal of Applied Psychology, 88(5), 879-903. 
*Pohjonen, T. (2001). Perceived work ability of home care workers in relation to individual and work-related factors in different age groups. Occupational Medicine, 51, 209-217.

Posthuma, R. A., \& Campion, M. A. (2009). Age stereotypes in the workplace: Common stereotypes, moderators, and future research directions. Journal of Management, 35(1), 158-188.

Pascoe, E. A., \& Smart Richman, L. (2009). Perceived discrimination and health: a metaanalytic review. Psychological Bulletin, 135(4), 531-554.

*Pranjić, N., Maleš-Bilić, L., Beganlić, A., \& Mustajbegović, J. (2006). Mobbing, stress, and work ability index among physicians in Bosnia and Herzegovina: survey study. Croatian Medical Journal, 47(5), 750-758.

*Punakallio, A., Lusa, S., \& Luukkonen, R. (2004). Functional, postural and perceived balance for predicting the work ability of firefighters. International Archives of Occupational and Environmental Health, 77(7), 482-490.

Radkiewicz, P., Widerszal-Bazyl, M., \& NEXT-Study Group. (2005). Psychometric properties of Work Ability Index in the light of comparative survey study. International Congress Series, 1280, 304-309.

*Ramada, J. M., Serra, C., Amick, B. C., Abma, F. I., Castaño, J. R., Pidemunt, G., \& Delclos, G. L. (2014). Reliability and validity of the work role functioning questionnaire (Spanish version). Journal of Occupational Rehabilitation, 24(4), 640-649. 
*Reeuwijk, K. G., Robroek, S. J., Niessen, M. A., Kraaijenhagen, R. A., Vergouwe, Y., \& Burdorf, A. (2015). The prognostic value of the work ability index for sickness absence among office workers. PloS One, 10(5), e0126969.

Rich, B. L., Lepine, J. A., \& Crawford, E. R. (2010). Job engagement: Antecedents and effects on job performance. Academy of Management Journal, 53(3), 617-635.

*Riedel, N., Müller, A., \& Ebener, M. (2015). Applying strategies of selection, optimization, and compensation to maintain work ability - a psychosocial resource complementing the Job Demand-Control Model? Results from the representative lid: A cohort study on work, age, and health in Germany. Journal of Occupational and Environmental Medicine, 57, 552-561.

*Riethmeister, V., Brouwer, S., van der Klink, J., \& Bültmann, U. (2015). Work, eat and sleep: towards a healthy ageing at work program offshore. BMC Public Health, 16(1), 134-145.

Rimm, E. B., Klatsky, A., Grobbee, D., \& Stampfer, M. J. (1996). Review of moderate alcohol consumption and reduced risk of coronary heart disease: is the effect due to beer, wine, or spirits?. British Medical Journal, 312, 731-736.

*Rineer, J. R. (2015). Supporting the aging workforce: The impact of psychosocial workplace characteristics on employees' work ability (Doctoral dissertation, Portland State University).

*Roelen, C. A., Heymans, M. W., Twisk, J. W., van der Klink, J. J., Groothoff, J. W., \& van Rhenen, W. (2014). Work Ability Index as tool to identify workers at risk of premature work exit. Journal of Occupational Rehabilitation, 24, 747-754. 
Rohwedder, S., \& Willis, R. J. (2010). Mental retirement. The Journal of Economic Perspectives, 24, 119-138. doi:10.1257/jep.24.1.119.

*Rolander, B., Jonker, D., Winkel, J., Sandsjö, L., Balogh, I., Svensson, E., \& Ekberg, K. (2013). Working conditions, health and productivity among dentists in Swedish public dental care-a prospective study during a 5-year period of rationalization. Ergonomics, 56, 1376-1386.

*Rongen, A., Robroek, S. J., Heijden, B. I., Schouteten, R., Hasselhorn, H. M., \& Burdorf, A. (2014). Influence of work-related characteristics and work ability on changing employer or leaving the profession among nursing staff. Journal of Nursing Management, 22(8), 1065-1075.

*Rongen, A., Robroek, S. J., Schaufeli, W., \& Burdorf, A. (2014). The contribution of work engagement to self-perceived health, work ability, and sickness absence beyond health behaviors and work-related factors. Journal of Occupational and Environmental Medicine, 56, 892-897.

Rosenthal, R. (1991). Meta-analytic procedures for social research. Newbury Park, CA: Sage.

*Rostamabadi, A., Zamanian, Z., \& Sedaghat, Z. (2017). Factors associated with work ability index (WAI) among intensive care units'(ICUs') nurses. Journal of Occupational Health, 59(2), 147-155.

*Rostamabadi, A., Mazloumi, A., \& Rahimi Foroushani, A. (2014). Work Ability Index (WAI) and Its Health-related Determinants among Iranian Farmers Working in Small Farm Enterprises. Journal of Occupational Health, 56, 478-484. 
Rudolph, C. W., Katz, I. M., Lavigne, K. N., \& Zacher, H. (2017). Job crafting: A metaanalysis of relationships with individual differences, job characteristics, and work outcomes. Journal of Vocational Behavior, 102, 112-138.

*Ruitenburg, M. M., Frings-Dresen, M. H., \& Sluiter, J. K. (2012). The prevalence of common mental disorders among hospital physicians and their association with self-reported work ability: a cross-sectional study. BMC Health Services Research, 12, 292-298.

Ryan, A. M., \& Ployhart, R. E. (2014). A century of selection. Annual Review of Psychology, 65, 693-717.

*Sahlin, E., Ahlborg, G., Matuszczyk, J. V., \& Grahn, P. (2014). Nature-based stress management course for individuals at risk of adverse health effects from workrelated stress — effects on stress related symptoms, workability and sick leave. International Journal of Environmental Research and Public Health, 11, $6,586-6,611$.

Salas, E., Tannenbaum, S. I., Kraiger, K., \& Smith-Jentsch, K. A. (2012). The science of training and development in organizations: What matters in practice. Psychological Science in the Public Interest, 13(2), 74-101.

*Salonen, P., Arola, H., Nygård, C. H., Huhtala, H., \& Koivisto, A. M. (2003). Factors associated with premature departure from working life among ageing food industry employees. Occupational Medicine, 53, 65-68.

*Saltychev, M., Laimi, K., Pentti, J., Kivimäki, M., \& Vahtera, J. (2014). Short-and longterm changes in perceived work ability after interdisciplinary rehabilitation of 
chronic musculoskeletal disorders: prospective cohort study among 854 rehabilitants. Clinical Rehabilitation, 28, 592-603.

Sameroff, A. (2010). A unified theory of development: A dialectic integration of nature and nurture. Child Development, 81(1), 6-22.

*Sampaio, R. F., Coelho, C. M., Barbosa, F. B., Mancini, M. C., \& Parreira, V. F. (2009). Work ability and stress in a bus transportation company in Belo Horizonte, Brazil. Ciência \& Saúde Coletiva, 14, 287-296.

Sanchez, J. I., \& Levine, E. L. (2012). The rise and fall of job analysis and the future of work analysis. Annual Review of Psychology, 63, 397-425.

*Sauni, R., Toivio, P., Pääkkönen, R., Malmström, J., \& Uitti, J. (2015). Work disability after diagnosis of hand-arm vibration syndrome. International Archives of Occupational and Environmental Health, 88(8), 1061-1068.

Scammacca, N., Roberts, G., \& Stuebing, K. K. (2014). Meta-analysis with complex research designs dealing with dependence from multiple measures and multiple group comparisons. Review of Educational Research, 84, 328-364.

Schaie, K. W., \& Willis, S. L. (1993). Age difference patterns of psychometric intelligence in adulthood: Generalizability within and across ability domains. Psychology and Aging, 8, 44-55.

Schane, R. E., Ling, P. M., \& Glantz, S. A. (2010). Health effects of light and intermittent smoking a review. Circulation, 121, 1518-1522.

*Schouten, L. S., Joling, C. I., van der Gulden, J. W., Heymans, M. W., Bültmann, U., \& Roelen, C. A. (2015). Screening manual and office workers for risk of long-term 
sickness absence: cut-off points for the Work Ability Index. Scandinavian Journal of Work, Environment \& Health, 41, 36-42.

Shultz, K. S. (2003). Bridge employment: Work after retirement. In G. A. Adams \& T. A. Beehr (Eds.), Retirement: Reasons, Processes, and Results (pp. 215-241). New York: Springer.

Shultz, K. S., Morton, K. R., \& Weckerle, J. R. (1998). The influence of push and pull factors on voluntary and involuntary early retirees' retirement decision and adjustment. Journal of Vocational Behavior, 53(1), 45-57.

Schaufeli, W. B., Bakker, A. B., \& Salanova, M. (2006). The measurement of work engagement with a short questionnaire: A cross-national study. Educational and Psychological Measurement, 66(4), 701-716

*Schulz, H., Zacher, H., \& Lippke, S. (2017). The importance of team health climate for health-related outcomes of white-collar workers. Frontiers in Psychology, 8, 74. doi: 10.3389/fpsyg.2017.00074.

*Seibt, R., Spitzer, S., Blank, M., \& Scheuch, K. (2009). Predictors of work ability in occupations with psychological stress. Journal of Public Health, 17, 9-18.

Sell, L. (2009). Predicting long-term sickness absence and early retirement pension from self-reported work ability. International Archives of Occupational and Environmental Health, 82(9), 1133-1138.

*Sell, L., Lund, H. L., Holtermann, A., \& Søgaard, K. (2014). The interactions between pain, pain-related fear of movement and productivity. Occupational Medicine, 64, 376-381. 
Sheldon, O. J., Dunning, D., \& Ames, D. R. (2014). Emotionally unskilled, unaware, and uninterested in learning more: Reactions to feedback about deficits in emotional intelligence. Journal of Applied Psychology, 99(1), 125-137.

Sherman, R. O., Chiang-Hanisko, L., \& Koszalinski, R. (2013). The ageing nursing workforce: A global challenge. Journal of Nursing Management, 21(7), 899-902.

*Shiri, R., Kaila-Kangas, L., Ahola, K., Kivekäs, T., Viikari-Juntura, E., Heliövaara, M., \& Leino-Arjas, P. (2013). The relation of co-occurring musculoskeletal pain and depressive symptoms with work ability. Journal of Occupational and Environmental Medicine, 55, 1,281-1,285.

Shirom, A., Shechter Gilboa, S., Fried, Y., \& Cooper, C. L. (2008). Gender, age and tenure as moderators of work-related stressors' relationships with job performance: A meta-analysis. Human Relations, 61(10), 1371-1398.

*Sluiter, J. K., \& Frings-Dresen, M. H. (2008). Quality of life and illness perception in working and sick-listed chronic RSI patients. International Archives of Occupational and Environmental Health, 81, 495-501.

*Smolander, J., Sörensen, L., Pekkonen, M., \& Alén, M. (2009). Muscle performance, work ability and physical functioning in middle-aged men. Occupational Medicine, 60(1), 78-80.

Snape, E., \& Redman, T. (2003). Too old or too young? The impact of perceived age discrimination. Human Resource Management Journal, 13(1), 78-89. 
Sonnentag, S., \& Fritz, C. (2007). The Recovery Experience Questionnaire: development and validation of a measure for assessing recuperation and unwinding from work. Journal of Occupational Health Psychology, 12(3), 204-221.

*Sörensen, L., Honkalehto, S., Kallinen, M., Pekkonen, M., Louhevaara, V., Smolander, J., \& Alén, M. (2007). Are cardiorespiratory fitness and walking performance associated with self-reported quality of life and work ability?. International Journal of Occupational Medicine and Environmental Health, 20, 257-264.

*Sörensen, L. E., Pekkonen, M. M., Männikkö, K. H., Louhevaara, V. A., Smolander, J., \& Alén, M. J. (2008). Associations between work ability, health-related quality of life, physical activity and fitness among middle-aged men. Applied Ergonomics, 39, 786-791.

Sörensen, L., Smolander, J., Louhevaara, V., Korhonen, O., \& Oja, P. (2000). Physical activity, fitness and body composition of Finnish police officers: a 15-year follow-up study. Occupational Medicine, 50(1), 3-10.

*Sormunen, E., Remes, J., Hassi, J., Pienimaki, T., \& Rintamaki, H., (2009). Factors associated with self-estimated work ability and musculoskeletal symptoms among male and female workers in cooled food-processing facilities. Industrial Health, $47,271-282$.

Spector, P. E. (1986). Perceived control by employees: A meta-analysis of studies concerning autonomy and participation at work. Human Relations, 39, 10051016. 
Spector, P. E., Dwyer, D. J., \& Jex, S. M. (1988). Relation of job stressors to affective, health, and performance outcomes: a comparison of multiple data sources. Journal of Applied Psychology, 73(1), 11-19.

Spreitzer, G. M. (1995). Psychological empowerment in the workplace: Dimensions, measurement, and validation. Academy of Management Journal, 38(5), 14421465.

Spreitzer, G. M., Cameron, L., \& Garrett, L. (2017). Alternative work arrangements: Two images of the new world of work. Annual Review of Organizational Psychology and Organizational Behavior, 4, 473-499.

*Stordeur, S., D'hoore, W., \& NEXT-Study Group. (2007). Organizational configuration of hospitals succeeding in attracting and retaining nurses. Journal of Advanced Nursing, 57(1), 45-58.

Sturman, M. C. (2003). Searching for the inverted U-shaped relationship between time and performance: Meta-analyses of the experience/performance, tenure/performance, and age/performance relationships. Journal of Management, 29(5), 609-640.

*Sugimura, H., \& Thériault, G. (2010). Impact of supervisor support on work ability in an IT company. Occupational Medicine, 60, 451-457.

*Sun, J., Buys, N., \& Wang, X. (2013). Depression in employees in privately owned enterprises in China: is it related to work environment and work ability?. International Journal of Environmental Research and Public Health, 10, 1152-1167. 
*Szlachta, E., Gawlik-Chmiel, B., \& Kallus, K. W. (2012). Do the long-term unemployed regard themselves as able to work?. Journal of Public Health, 20, 505-511.

*Tavakoli-Fard, N., Mortazavi, S. A., Kuhpayehzadeh, J., \& Nojomi, M. (2016). Quality of life, work ability and other important indicators of women's occupational health. International Journal of Occupational Medicine \& Environmental Health, 29(1), 77-84.

Tierney, P., \& Farmer, S. M. (2002). Creative self-efficacy: Its potential antecedents and relationship to creative performance. Academy of Management Journal, 45(6), $1137-1148$.

Tierney, P., \& Farmer, S. M. (2011). Creative self-efficacy development and creative performance over time. Journal of Applied Psychology, 96(2), 277-293.

Toossi, M. (2015). Labor force projections to 2024: The labor force is growing, but slowly. Monthly Labor Review, 1-36. Retrieved from http://www.bls.gov/opub/mlr/2015/article/pdf/labor-force-projections-to2024.pdf.

*Torp, S., Nielsen, R. A., Gudbergsson, S. B., \& Dahl, A. A. (2012). Worksite adjustments and work ability among employed cancer survivors. Supportive Care in Cancer, 20, 2,149-2,156.

Truxillo, D. M., Cadiz, D. M., \& Hammer, L. B. (2015). Supporting the aging workforce: A review and recommendations for workplace intervention research. Annual Review of Organizational Psychology and Organizational Behavior, 2(1), 351381. 
*Tuomi, K., Huuhtanen, P., Nykyri, E., \& Ilmarinen, J. (2001). Promotion of work ability, the quality of work and retirement. Occupational Medicine-Oxford, 51, 318-324.

Tuomi, K., Vanhala, S., Nykyri, E., \& Janhonen, M. (2004). Organizational practices, work demands and the well-being of employees: a follow-up study in the metal industry and retail trade. Occupational Medicine, 54, 115-121. Doi:

10.1093/occmed/kqh005

Uniform guidelines on employee selection procedures, 43 Fed. Reg. 38290-38315 (1978).

United States Bureau of Labor Statistics (2017). Employment projections 2016-2026.

Bureau of Labor Statistics. Retrieved from:

https://www.bls.gov/news.release/pdf/ecopro.pdf.

*van de Vijfeijke, H., Leijten, F. R., Ybema, J. F., van den Heuvel, S. G., Robroek, S. J., van der Beek, A. J., \& Taris, T. W. (2013). Differential effects of mental and physical health and coping style on work ability: a 1-year follow-up study among aging workers. Journal of Occupational and Environmental Medicine, 55(10), $1238-1243$.

*van den Berg, T. I., Alavinia, S. M., Bredt, F. J., Lindeboom, D., Elders, L. A., \& Burdorf, A. (2008). The influence of psychosocial factors at work and life style on health and work ability among professional workers. International Archives of Occupational and Environmental Health, 81, 1,029-1,036.

*van den Berg, T. I., Robroek, S. J., Plat, J. F., Koopmanschap, M. A., \& Burdorf, A. (2011). The importance of job control for workers with decreased work ability to 
remain productive at work. International Archives of Occupational and Environmental Health, 84(6), 705-712.

*van Holland, B. J., Soer, R., de Boer, M. R., Reneman, M. F., \& Brouwer, S. (2015). Workers' health surveillance in the meat processing industry: work and health indicators associated with work ability. Journal of Occupational Rehabilitation, 25, 618-626.

*van Muijen, P., Duijts, S. F., Bonefaas-Groenewoud, K., van der Beek, A. J., \& Anema, J. R. (2014). Factors associated with work disability in employed cancer survivors at 24-month sick leave. BMC Cancer, 14(1), 236-246.

*Vasconcelos, S. P., Fischer, F. M., Reis, A. O. A., \& Moreno, C. R. D. C. (2011). Factors associated with work ability and perception of fatigue among nursing personnel from Amazonia. Revista Brasileira de Epidemiologia, 14, 688-697.

*Vastamäki, J., Wolff, H. G., Paul, K. I., \& Moser, K. (2014). Sense of coherence mediates the effects of low work ability on mental distress during unemployment. Journal of Workplace Behavioral Health, 29, 317-332.

*Vastamäki, J., Moser, K., \& Paul, K. I. (2009). How stable is sense of coherence? Changes following an intervention for unemployed individuals. Scandinavian Journal of Psychology, 50, 161-171.

*Vedovato, T. G., \& Monteiro, I. (2014). Health conditions and factors related to the work ability of teachers. Industrial Health, 52(2), 121-128. 
Velez, B. L., \& Moradi, B. (2012). Workplace support, discrimination, and personorganization fit: Tests of the theory of work adjustment with LGB individuals. Journal of Counseling Psychology, 59(3), 399.

*Verhoef, J. A., Miedema, H. S., Bramsen, I., \& Roebroeck, M. E. (2012). Using the work limitations questionnaire in patients with a chronic condition in the Netherlands. Journal of Occupational and Environmental Medicine, 54(10), 1293-1299.

*Vindholmen, S., Høigaard, R., Espnes, G. A., \& Seiler, S. (2014). Return to work after vocational rehabilitation: does mindfulness matter?. Psychology Research and Behavior Management, 7, 77-88.

*Viotti, S., Guidetti, G., Loera, B., Martini, M., Sottimano, I., \& Converso, D. (2017). Stress, work ability, and an aging workforce: A study among women aged 50 and over. International Journal of Stress Management, 24(1), 98-121.

*von Bonsdorff, M. E., Huuhtanen, P., Tuomi, K., \& Seitsamo, J. (2009). Predictors of employees' early retirement intentions: an 11-year longitudinal study. Occupational Medicine, 60, 94-100.

*von Bonsdorff, M. B., Seitsamo, J., Ilmarinen, J., Nygård, C. H., von Bonsdorff, M. E., \& Rantanen, T. (2011). Work ability in midlife as a predictor of mortality and disability in later life: a 28-year prospective follow-up study. Canadian Medical Association Journal, 183, E235-E242.

*von Bonsdorff, M. E., Vanhala, S., Seitsamo, J., Janhonen, M., \& Husman, P. (2010). Employee well-being, early-retirement intentions, and company 
performance. Journal of Occupational and Environmental Medicine, 52(12), 1255-1261.

*von Bonsdorff, M. E., von Bonsdorff, M. B., Zhou, Z. E., Kauppinen, M., Miettinen, M., Rantanen, T., \& Vanhala, S. (2014). Organizational justice, selection, optimization with compensation, and nurses' work ability. Journal of Occupational and Environmental Medicine, 56(3), 326-330.

*von Bonsdorff, M. E., Kokko, K., Seitsamo, J., von Bonsdorff, M. B., Nygård, C. H., Ilmarinen, J., \& Rantanen, T. (2011). Work strain in midlife and 28-year work ability trajectories. Scandinavian Journal of Work, Environment \& Health, 455463.

*WåHlin-Norgren, C., Ekberg, K., \& Öberg, B. (2011). Is an expert diagnosis enough for assessment of sick leave for employees with musculoskeletal and mental disorders?. Disability and Rqehabilitation, 33(13-14), 1147-1156.

*Wagenaar, A. F., Kompier, M. A., Houtman, I. L., van den Bossche, S. N., \& Taris, T. W. (2015). Who gets fired, who gets re-hired: the role of workers' contract, age, health, work ability, performance, work satisfaction and employee investments. International Archives of Occupational and Environmental Health, 88, 321-334.

*Walker, E. J., Jackson, C. A., Egan, H. H., \& Tonkin, M. (2015). Workability and mental wellbeing among therapeutic prison officers. Occupational Medicine, 65, $549-551$. 
*Walsh, I. A., Oishi, J., \& Coury, H. J. (2008). Clinical and functional aspects of workrelated musculoskeletal disorders among active workers. Revista de Saude Publica, 42, 108-116.

Wang, G., Oh, I. S., Courtright, S. H., \& Colbert, A. E. (2011). Transformational leadership and performance across criteria and levels: A meta-analytic review of 25 years of research. Group \& Organization Management, 36(2), 223-270.

*Weigl, M., Müller, A., Hornung, S., Zacher, H., \& Angerer, P. (2013). The moderating effects of job control and selection, optimization, and compensation strategies on the age-work ability relationship. Journal of Organizational Behavior, 34, 607628.

*Weng, L. C., Huang, H. L., Wang, Y. W., Lee, W. C., Chen, K. H., \& Yang, T. Y. (2014). The effect of self-efficacy, depression and symptom distress on employment status and leisure activities of liver transplant recipients. Journal of Advanced Nursing, 70, 1573-1583.

*Wilke, C., Ashton, P., Elis, T., Biallas, B., \& Froböse, I. (2015). Analysis of work ability and work-related physical activity of employees in a medium-sized business. BMC Research Notes, 8(1), 803-808.

Xanthopoulou, D., Bakker, A. B., Demerouti, E., \& Schaufeli, W. B. (2007). The role of personal resources in the job demands-resources model. International Journal of Stress Management, 14, 121-141. 
*Yong, M., Nasterlack, M., Pluto, R. P., Elmerich, K., Karl, D., \& Knauth, P. (2010). Is health, measured by work ability index, affected by 12-hour rotating shift schedules?. Chronobiology International, 27, 1,135-1,148.

Yuan, A. S. V. (2007). Perceived age discrimination and mental health. Social Forces, 86(1), 291-311.

Zacher, H., Feldman, D. C., \& Schulz, H. (2014). Age, occupational strain, and wellbeing: A person-environment fit perspective. In The role of demographics in occupational stress and well being (pp. 83-111). Emerald Group Publishing Limited.

*Zacher, H. \& Rudolph, C. W. (in press). Just a mirage: On the incremental predictive validity of subjective age. Work, Aging, and Retirement.

Zaniboni, S. (2015). The interaction between older workers' personal resources and perceived age discrimination affects the desired retirement age and the expected adjustment. Work, Aging and Retirement, 1, 266-273.

Zapata-Phelan, C. P., Colquitt, J. A., Scott, B. A., \& Livingston, B. (2009). Procedural justice, interactional justice, and task performance: The mediating role of intrinsic motivation. Organizational Behavior and Human Decision Processes, 108(1), 93105.

Zhan, Y., Wang, M., Liu, S., \& Shultz, K. S. (2009). Bridge employment and retirees' health: A longitudinal investigation. Journal of Occupational Health Psychology, 14, 374-389.

*Indicates that the study was included in the meta-analysis from Study 1. 
Appendix Study Level Results from Meta-Analysis

\begin{tabular}{|c|c|c|c|c|c|}
\hline Citation & $\begin{array}{l}\text { Occupation } \\
\text { Type }\end{array}$ & $\begin{array}{c}\text { WA } \\
\text { Measure }\end{array}$ & Correlate & $n$ & $r$ \\
\hline \multirow{6}{*}{$\begin{array}{l}\text { Abdolalizadeh, M., } \\
\text { Arastoo, A. A., } \\
\text { Ghsemzadeh, R., } \\
\text { Montazeri, A., Ahmadi, K., } \\
\text { \& Azizi, A. (2012). The } \\
\text { psychometric properties of } \\
\text { an Iranian translation of the } \\
\text { Work Ability Index (WAI) } \\
\text { questionnaire. Journal of } \\
\text { Occupational } \\
\text { Rehabilitation, 22(3), 401- } \\
\text { 408. }\end{array}$} & \multirow[t]{6}{*}{$\begin{array}{l}\text { Nursing/Hea } \\
\text { lthcare }\end{array}$} & \multirow[t]{6}{*}{ WAI } & $\begin{array}{l}\text { General } \\
\text { Health }\end{array}$ & 236 & .723 \\
\hline & & & $\begin{array}{l}\text { Mental } \\
\text { Health }\end{array}$ & 236 & .420 \\
\hline & & & $\begin{array}{l}\text { Physical } \\
\text { Health }\end{array}$ & 236 & .620 \\
\hline & & & $\begin{array}{l}\text { Pain/Muscul } \\
\text { oskeletal } \\
\text { Symptoms }\end{array}$ & 236 & .580 \\
\hline & & & $\begin{array}{l}\text { Physical } \\
\text { Ability }\end{array}$ & 236 & .650 \\
\hline & & & Fatigue & 236 & -.510 \\
\hline \multirow{5}{*}{$\begin{array}{l}\text { Abma, F. I., Brouwer, S., } \\
\text { de Vries, H. J., Arends, I., } \\
\text { Robroek, S. J., Cuijpers, M. } \\
\text { P., \& van der Klink, J. J. } \\
\text { (2016). The capability set } \\
\text { for work: development and } \\
\text { validation of a new } \\
\text { questionnaire. } \\
\text { Scandinavian Journal of } \\
\text { Work Environment \& } \\
\text { Health, 42(1), 34-42. }\end{array}$} & \multirow[t]{5}{*}{ Mixed/Other } & \multirow[t]{5}{*}{ PWA** } & $\begin{array}{l}\text { Mental/Emot } \\
\text { ional } \\
\text { Demands }\end{array}$ & 1157 & -.149 \\
\hline & & & $\begin{array}{l}\text { Physical } \\
\text { Demands }\end{array}$ & 1157 & -.188 \\
\hline & & & $\begin{array}{l}\text { Previous } \\
\text { Absenteeism }\end{array}$ & 1157 & -.294 \\
\hline & & & Age & 1157 & -.080 \\
\hline & & & $\begin{array}{l}\text { Job } \\
\text { Performance }\end{array}$ & 1157 & .469 \\
\hline \multirow{2}{*}{$\begin{array}{l}\text { Abma, F. I., van der Klink, } \\
\text { J. J., \& Bültmann, U. } \\
\text { (2013). The work role } \\
\text { functioning questionnaire } \\
2.0 \text { (dutch version): } \\
\text { Examination of its }\end{array}$} & \multirow[t]{2}{*}{ Mixed/Other } & \multirow[t]{2}{*}{ PWA* } & $\begin{array}{l}\text { Mental/Emot } \\
\text { ional } \\
\text { Demands }\end{array}$ & 275 & -.323 \\
\hline & & & $\begin{array}{l}\text { Physical } \\
\text { Demands }\end{array}$ & 275 & -.433 \\
\hline
\end{tabular}




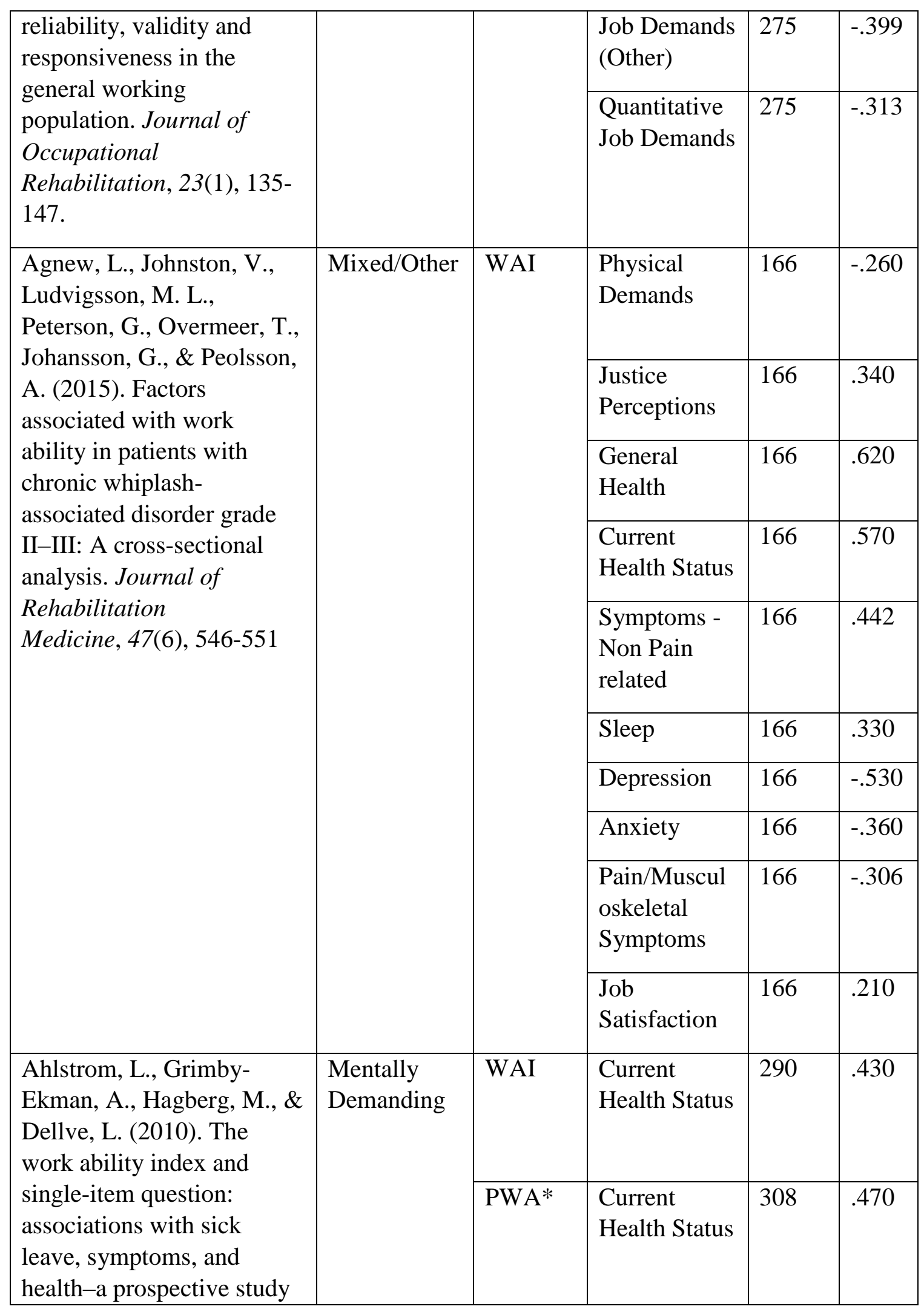




\begin{tabular}{|c|c|c|c|c|c|}
\hline $\begin{array}{l}\text { of women on long-term } \\
\text { sick leave. Scandinavian } \\
\text { Journal of Work, } \\
\text { Environment \& Health, } \\
36(5), 404-412 \text {. }\end{array}$ & & & & & \\
\hline \multirow{17}{*}{$\begin{array}{l}\text { Airila, A., Hakanen, J., } \\
\text { Punakallio, A., Lusa, S., \& } \\
\text { Luukkonen, R. (2012). Is } \\
\text { work engagement related to } \\
\text { work ability beyond } \\
\text { working conditions and } \\
\text { lifestyle factors?. } \\
\text { International Archives of } \\
\text { Occupational and } \\
\text { Environmental Health, } \\
\text { 85(8), 915-925. } \dagger\end{array}$} & \multirow[t]{11}{*}{ Mixed/Other } & \multirow[t]{11}{*}{ PWA* } & $\begin{array}{l}\text { Physical } \\
\text { Demands }\end{array}$ & 403 & -.060 \\
\hline & & & Job Demands & 403 & -.327 \\
\hline & & & $\begin{array}{l}\text { Supervisor } \\
\text { Support }\end{array}$ & 403 & .163 \\
\hline & & & $\begin{array}{l}\text { Task } \\
\text { Resources }\end{array}$ & 403 & .166 \\
\hline & & & Sleep & 403 & .368 \\
\hline & & & BMI & 403 & -.130 \\
\hline & & & Exercise & 403 & .195 \\
\hline & & & Drinking & 403 & -.075 \\
\hline & & & Smoking & 403 & -.080 \\
\hline & & & Age & 403 & -.245 \\
\hline & & & $\begin{array}{l}\text { Work } \\
\text { Motivation }\end{array}$ & 403 & .260 \\
\hline & \multirow[t]{6}{*}{ Mixed/Other } & \multirow[t]{6}{*}{ WAI } & $\begin{array}{l}\text { Physical } \\
\text { Demands }\end{array}$ & 403 & -.110 \\
\hline & & & Job Demands & 403 & -.300 \\
\hline & & & $\begin{array}{l}\text { Supervisor } \\
\text { Support }\end{array}$ & 403 & .190 \\
\hline & & & $\begin{array}{l}\text { Task } \\
\text { Resources }\end{array}$ & 403 & .290 \\
\hline & & & Sleep & 403 & .370 \\
\hline & & & BMI & 403 & -.150 \\
\hline
\end{tabular}




\begin{tabular}{|c|c|c|c|c|c|}
\hline & & & $\begin{array}{l}\text { Work } \\
\text { Motivation }\end{array}$ & 403 & .410 \\
\hline & & & Exercise & 403 & .150 \\
\hline & & & Drinking & 403 & -.070 \\
\hline & & & Smoking & 403 & -.100 \\
\hline & & & Age & 403 & -.330 \\
\hline $\begin{array}{l}\text { Airila, A., Hakanen, J. J., } \\
\text { Luukkonen, R., Lusa, S., \& } \\
\text { Punakallio, A. (2013). } \\
\text { Positive and Negative }\end{array}$ & Mixed/Other & WAI & $\begin{array}{l}\text { General } \\
\text { Health }\end{array}$ & 360 & .600 \\
\hline $\begin{array}{l}\text { Mood Trajectories and } \\
\text { Their Relationship With } \\
\text { Work Ability, Self-Rated } \\
\text { Health, and Life } \\
\text { Satisfaction: A 13-Year } \\
\text { Follow-Up Study. Journal } \\
\text { of Occupational and } \\
\text { Environmental } \\
\text { Medicine, 55(7), 779-785.† }\end{array}$ & & & Fatigue & 360 & -.278 \\
\hline $\begin{array}{l}\text { Airila, A., Hakanen, J. J., } \\
\text { Schaufeli, W. B., }\end{array}$ & Mixed/Other & PWA & $\begin{array}{l}\text { Workplace } \\
\text { Mistreatment }\end{array}$ & 403 & -.075 \\
\hline $\begin{array}{l}\text { A., \& Lusa, S. (2014). Are } \\
\text { job and personal resources }\end{array}$ & & & $\begin{array}{l}\text { Core Self } \\
\text { Evaluations }\end{array}$ & 403 & .195 \\
\hline $\begin{array}{l}\text { associated with work } \\
\text { ability } 10 \text { years later? The } \\
\text { mediating role of work } \\
\text { engagement. Work \& } \\
\text { Stress, } 28(1), 87-105 . \dagger\end{array}$ & & & $\begin{array}{l}\text { Work } \\
\text { Motivation }\end{array}$ & & \\
\hline $\begin{array}{l}\text { Alavinia, S. M., Van Den } \\
\text { Berg, T. I., Van } \\
\text { Duivenbooden, C., Elders, } \\
\text { L. A., \& Burdorf, A. } \\
\text { (2009). Impact of work- } \\
\text { related factors, lifestyle, } \\
\text { and work ability on } \\
\text { sickness absence among }\end{array}$ & Blue Collar & WAI & $\begin{array}{l}\text { Future } \\
\text { Absenteeism }\end{array}$ & 5872 & -.103 \\
\hline
\end{tabular}




\begin{tabular}{|c|c|c|c|c|c|}
\hline $\begin{array}{l}\text { Dutch construction } \\
\text { workers. Scandinavian } \\
\text { Journal of Work, } \\
\text { Environment \& Health, } \\
\text { 35(5), 325-333. }\end{array}$ & & & & & \\
\hline $\begin{array}{l}\text { Alavinia, S. M., De Boer, } \\
\text { A. G. E. M., Van } \\
\text { Duivenbooden, J. C., } \\
\text { Frings-Dresen, M. H. W., } \\
\text { \& Burdorf, A. (2009). } \\
\text { Determinants of work } \\
\text { ability and its predictive } \\
\text { value for } \\
\text { disability. Occupational } \\
\text { Medicine, 59(1), 32-37. }\end{array}$ & Blue Collar & WAI & $\begin{array}{l}\text { Future } \\
\text { Disability }\end{array}$ & 606 & -.543 \\
\hline \multirow{2}{*}{$\begin{array}{l}\text { Arandjelovic, M., Nikolic, } \\
\text { M., \& Stamenkovic, S. } \\
\text { (2010). Relationship } \\
\text { between burnout, quality of } \\
\text { life, and work ability } \\
\text { index-directions in } \\
\text { prevention. The Scientific } \\
\text { World Journal, 10, 766- } \\
777 .\end{array}$} & \multirow[t]{2}{*}{ Blue Collar } & \multirow[t]{2}{*}{ WAI } & Age & 489 & -.034 \\
\hline & & & Burnout & 489 & -.154 \\
\hline \multirow{6}{*}{$\begin{array}{l}\text { Assunção, A. A., Sampaio, } \\
\text { R. F., \& Nascimento, L. } \\
\text { (2010). Actions in small } \\
\text { companies to promote } \\
\text { occupational health: the } \\
\text { case of the food and } \\
\text { beverage sector. Brazilian } \\
\text { Journal of Physical } \\
\text { Therapy, 14(1), 52-59. }\end{array}$} & \multirow[t]{6}{*}{ Mixed/Other } & \multirow[t]{6}{*}{ WAI } & $\begin{array}{l}\text { Social } \\
\text { Support } \\
\text { (non-work) }\end{array}$ & 378 & .120 \\
\hline & & & Sleep & 378 & .308 \\
\hline & & & $\begin{array}{l}\text { Mental } \\
\text { Health }\end{array}$ & 378 & .356 \\
\hline & & & $\begin{array}{l}\text { Physical } \\
\text { Health }\end{array}$ & 378 & .330 \\
\hline & & & $\begin{array}{l}\text { Pain/Muscul } \\
\text { oskeletal } \\
\text { Symptoms }\end{array}$ & 378 & -.485 \\
\hline & & & Age & 378 & -.254 \\
\hline
\end{tabular}




\begin{tabular}{|c|c|c|c|c|c|}
\hline & & & Fatigue & 378 & -.304 \\
\hline \multirow{5}{*}{$\begin{array}{l}\text { Attarchi, M., Ghaffari, M., } \\
\text { Abdi, A., } \\
\text { Mirzamohammadi, E., } \\
\text { Seyedmehdi, S. M., } \\
\text { Rahimpour, F., \& } \\
\text { Mohammadi, S. (2014). } \\
\text { Assessment of the } \\
\text { Relationship between } \\
\text { Physical Working } \\
\text { Conditions and Different } \\
\text { Levels of Work } \\
\text { Ability. Global Journal of } \\
\text { Health Science, 6(4), 213- } \\
\text { 220. }\end{array}$} & \multirow[t]{5}{*}{ Blue Collar } & \multirow[t]{5}{*}{ WAI } & $\begin{array}{l}\text { Physical } \\
\text { Demands }\end{array}$ & 641 & -.087 \\
\hline & & & BMI & 641 & -.259 \\
\hline & & & Exercise & 641 & .194 \\
\hline & & & Smoking & 641 & -.146 \\
\hline & & & Age & 641 & -.122 \\
\hline $\begin{array}{l}\text { Barnes-Farrell, J. L., } \\
\text { Rumery, S. M., \& Swody, } \\
\text { C. A. (2002). How do } \\
\text { concepts of age relate to } \\
\text { work and off-the-job } \\
\text { stresses and strains? A field } \\
\text { study of health care } \\
\text { workers in five } \\
\text { nations. Experimental } \\
\text { Aging Research, 28(1), 87- } \\
\text { 98. }\end{array}$ & $\begin{array}{l}\text { Nurses/Healt } \\
\text { hcare }\end{array}$ & PWA & Age & 880 & . 160 \\
\hline \multirow{6}{*}{$\begin{array}{l}\text { Beltrame, M. T., Magnago, } \\
\text { T. S. B. D. S., Kirchhof, A. } \\
\text { L. C., Marconato, C. D. S., } \\
\text { \& Morais, B. X. (2014). } \\
\text { Work ability in hospital } \\
\text { housekeeping services and } \\
\text { associated factors. Revista } \\
\text { Gaucha de } \\
\text { Enfermagem, 35(4), 49-57. }\end{array}$} & \multirow[t]{6}{*}{$\begin{array}{l}\text { Physically } \\
\text { Demanding }\end{array}$} & \multirow[t]{6}{*}{ WAI } & $\begin{array}{l}\text { General } \\
\text { Health }\end{array}$ & 157 & .148 \\
\hline & & & $\begin{array}{l}\text { Mental } \\
\text { Health }\end{array}$ & 157 & .315 \\
\hline & & & BMI & 157 & -.046 \\
\hline & & & Smoking & 130 & -.009 \\
\hline & & & Drinking & 157 & .240 \\
\hline & & & Age & 157 & -.095 \\
\hline
\end{tabular}




\begin{tabular}{|c|c|c|c|c|c|}
\hline \multirow{5}{*}{$\begin{array}{l}\text { Bennett, A. I., Hanley, J., } \\
\text { Buckle, P., \& Bridger, R. S. } \\
\text { (2011). Work demands } \\
\text { during firefighting training: } \\
\text { does age } \\
\text { matter?. Ergonomics, 54(6) } \\
\text {, 555-564. }\end{array}$} & \multirow[t]{5}{*}{ Mixed/Other } & \multirow[t]{5}{*}{ WAI } & $\begin{array}{l}\text { Physical } \\
\text { Health }\end{array}$ & 48 & .550 \\
\hline & & & $\begin{array}{l}\text { Physical } \\
\text { Ability }\end{array}$ & 48 & .390 \\
\hline & & & BMI & 48 & .530 \\
\hline & & & Exercise & 48 & .294 \\
\hline & & & Age & 48 & -.300 \\
\hline \multirow{5}{*}{$\begin{array}{l}\text { Bertilsson, M., Vaez, M., } \\
\text { Waern, M., Ahlborg Jr, G., } \\
\text { \& Hensing, G. (2014). A } \\
\text { prospective study on self- } \\
\text { assessed mental well-being } \\
\text { and work capacity as } \\
\text { determinants of all-cause } \\
\text { sickness absence. Journal } \\
\text { of Occupational } \\
\text { Rehabilitation, 25(1), 52- } \\
64 .\end{array}$} & \multirow[t]{5}{*}{ Mixed/Other } & \multirow[t]{5}{*}{ PWA } & $\begin{array}{l}\text { Core Self } \\
\text { Evaluations }\end{array}$ & 2502 & .292 \\
\hline & & & $\begin{array}{l}\text { Symptoms - } \\
\text { Non Pain } \\
\text { related }\end{array}$ & 2502 & .352 \\
\hline & & & $\begin{array}{l}\text { Pain/Muscul } \\
\text { oskeletal } \\
\text { Symptoms }\end{array}$ & 2502 & -.260 \\
\hline & & & $\begin{array}{l}\text { Future } \\
\text { Absenteeism }\end{array}$ & 2408 & -.129 \\
\hline & & & Fatigue & 2502 & -.320 \\
\hline \multirow{3}{*}{$\begin{array}{l}\text { Bethge, M., \& } \\
\text { Radoschewski, F. M. } \\
\text { (2010). Physical and } \\
\text { psychosocial work } \\
\text { stressors, health-related } \\
\text { control beliefs and work } \\
\text { ability: cross-sectional } \\
\text { findings from the German } \\
\text { Sociomedical Panel of } \\
\text { Employees. International } \\
\text { Archives of Occupational } \\
\text { and Environmental } \\
\text { Health, 83(3), 241-250. } \dagger\end{array}$} & \multirow[t]{3}{*}{ Mixed/Other } & \multirow[t]{3}{*}{ WAI } & $\begin{array}{l}\text { Physical } \\
\text { Demands }\end{array}$ & 1348 & -.375 \\
\hline & & & & & \\
\hline & & & $\begin{array}{l}\text { Justice } \\
\text { Perceptions }\end{array}$ & 1348 & .329 \\
\hline $\begin{array}{l}\text { Bethge, M., \& } \\
\text { Radoschewski, F. M. } \\
\text { (2012). Adverse effects of } \\
\text { effort-reward imbalance on }\end{array}$ & Mixed/Other & WAI & $\begin{array}{l}\text { Mental } \\
\text { Health }\end{array}$ & 603 & .610 \\
\hline
\end{tabular}




\begin{tabular}{|c|c|c|c|c|c|}
\hline $\begin{array}{l}\text { work ability: longitudinal } \\
\text { findings from the German } \\
\text { Sociomedical Panel of } \\
\text { Employees. International } \\
\text { Journal of Public } \\
\text { Health, 57(5), 797-805. } \dagger\end{array}$ & & & $\begin{array}{l}\text { Physical } \\
\text { Health }\end{array}$ & 603 & .607 \\
\hline \multirow{6}{*}{$\begin{array}{l}\text { Bethge, M., Radoschewski, } \\
\text { F. M., \& Gutenbrunner, C. } \\
\text { (2012). Effort-reward } \\
\text { imbalance and work ability: } \\
\text { cross-sectional and } \\
\text { longitudinal findings from } \\
\text { the Second German } \\
\text { Sociomedical Panel of } \\
\text { Employees. BMC Public } \\
\text { Health, 12(1), 875. } \dagger\end{array}$} & \multirow[t]{6}{*}{ White Collar } & \multirow[t]{6}{*}{ WAI } & $\begin{array}{l}\text { Physical } \\
\text { Demands }\end{array}$ & 1501 & -.129 \\
\hline & & & Job Demands & 1501 & -.260 \\
\hline & & & Job Control & 1501 & .303 \\
\hline & & & $\begin{array}{l}\text { Justice } \\
\text { Perceptions }\end{array}$ & 1501 & .434 \\
\hline & & & $\begin{array}{l}\text { Health } \\
\text { Behaviors }\end{array}$ & 1501 & .042 \\
\hline & & & Age & 1501 & -.013 \\
\hline \multirow{8}{*}{$\begin{array}{l}\text { Bethge, M., Radoschewski, } \\
\text { F. M., \& Gutenbrunner, C. } \\
\text { (2012). The Work Ability } \\
\text { Index as a screening tool to } \\
\text { identify the need for } \\
\text { rehabilitation: longitudinal } \\
\text { findings from the Second } \\
\text { German Sociomedical } \\
\text { Panel of Employees. } \\
\text { Journal of Rehabilitation } \\
\text { Medicine, 44(11), 980- } \\
\text { 987. } \dagger\end{array}$} & \multirow[t]{8}{*}{ White Collar } & \multirow[t]{8}{*}{ WAI } & $\begin{array}{l}\text { General } \\
\text { Health }\end{array}$ & 1036 & .425 \\
\hline & & & $\begin{array}{l}\text { Mental } \\
\text { Health }\end{array}$ & 1036 & .435 \\
\hline & & & $\begin{array}{l}\text { Pain/Muscul } \\
\text { oskeletal } \\
\text { Symptoms }\end{array}$ & 1036 & -.480 \\
\hline & & & $\begin{array}{l}\text { Physical } \\
\text { Health }\end{array}$ & 1036 & .495 \\
\hline & & & Fatigue & 1036 & -.530 \\
\hline & & & $\begin{array}{l}\text { Retirement } \\
\text { Intentions }\end{array}$ & 1036 & -.710 \\
\hline & & & $\begin{array}{l}\text { Future } \\
\text { Absenteeism }\end{array}$ & 1036 & -.550 \\
\hline & & & $\begin{array}{l}\text { Future } \\
\text { Disability } \\
\text { Status }\end{array}$ & 1036 & -.420 \\
\hline
\end{tabular}




\begin{tabular}{|c|c|c|c|c|c|}
\hline \multirow{5}{*}{$\begin{array}{l}\text { Bordin, G., Padalino, M. } \\
\text { A., Perentaler, S., Castaldi, } \\
\text { B., Maschietto, N., } \\
\text { Michieli, P., \& Milanesi, O. } \\
\text { (2015). Clinical profile and } \\
\text { quality of life of adult } \\
\text { patients after the Fontan } \\
\text { procedure. Pediatric } \\
\text { Cardiology, 36(6), 1261- } \\
1269 .\end{array}$} & \multirow[t]{5}{*}{ Mixed/Other } & \multirow[t]{5}{*}{ WAI } & $\begin{array}{l}\text { General } \\
\text { Health }\end{array}$ & 35 & .611 \\
\hline & & & $\begin{array}{l}\text { Mental } \\
\text { Health }\end{array}$ & 35 & .502 \\
\hline & & & $\begin{array}{l}\text { Physical } \\
\text { Health }\end{array}$ & 35 & .564 \\
\hline & & & $\begin{array}{l}\text { Pain/Muscul } \\
\text { oskeletal } \\
\text { Symptoms }\end{array}$ & 35 & -.534 \\
\hline & & & Fatigue & 35 & -.496 \\
\hline $\begin{array}{l}\text { Börner, K., Scherf, C., } \\
\text { Leitner-Mai, B., \& } \\
\text { Spanner-Ulmer, B. (2011). } \\
\text { Field study of age- } \\
\text { differentiated strain for } \\
\text { assembly line workers in } \\
\text { the automotive industry. } \\
\text { Work, 41, 5160-5166. }\end{array}$ & Blue Collar & WAI & Age & 23 & .103 \\
\hline $\begin{array}{l}\text { Boström, M., Sluiter, J. K., } \\
\text { Hagberg, M., \& Grimby- } \\
\text { Ekman, A. (2016). } \\
\text { Opportunities for recovery } \\
\text { at work and excellent work } \\
\text { ability-a cross-sectional } \\
\text { population study among } \\
\text { young workers. BMC } \\
\text { Public Health, 16(1), 985- } \\
\text { 996. }\end{array}$ & Mixed/Other & PWA & Job Demands & 2351 & -.050 \\
\hline $\begin{array}{l}\text { Bridger, R. S., \& Bennett, } \\
\text { A. I. (2011). Age and BMI } \\
\text { interact to determine work } \\
\text { ability in } \\
\text { seafarers. Occupational } \\
\text { Medicine, 61(3), 157-162. }\end{array}$ & Mixed/Other & WAI & Age & 38 & -.330 \\
\hline $\begin{array}{l}\text { Bridger, R. S., Dobson, K., } \\
\text { \& Davison, H. (2016). }\end{array}$ & Mixed/Other & PWA** & $\begin{array}{l}\text { Workplace } \\
\text { Mistreatment }\end{array}$ & 602 & -.294 \\
\hline
\end{tabular}




\begin{tabular}{|c|c|c|c|c|c|}
\hline \multirow{7}{*}{$\begin{array}{l}\text { Using the HSE stress } \\
\text { indicator tool in a military } \\
\text { context. Ergonomics, } 59(2) \text {, } \\
\text { 195-206. }\end{array}$} & & & $\begin{array}{l}\text { Mental/Emot } \\
\text { ional } \\
\text { Demands }\end{array}$ & 432 & -.351 \\
\hline & & & $\begin{array}{l}\text { Job Demands } \\
\text { (Other) }\end{array}$ & 423 & -.295 \\
\hline & & & $\begin{array}{l}\text { Supervisor } \\
\text { Support }\end{array}$ & 485 & .388 \\
\hline & & & Job Control & 391 & .229 \\
\hline & & & $\begin{array}{l}\text { Coworker } \\
\text { Support }\end{array}$ & 538 & .328 \\
\hline & & & $\begin{array}{l}\text { Current } \\
\text { Health Status }\end{array}$ & 1025 & .090 \\
\hline & & & Age & 1025 & -.090 \\
\hline \multirow{3}{*}{$\begin{array}{l}\text { Bugajska, J., \& Sagan, A. } \\
\text { (2014). Chronic } \\
\text { Musculoskeletal Disorders } \\
\text { as Risk Factors for } \\
\text { Reduced Work Ability in } \\
\text { Younger and Ageing } \\
\text { Workers. International } \\
\text { Journal of Occupational } \\
\text { Safety and Ergonomics, } \\
\text { 20(4), 607-615. }\end{array}$} & \multirow[t]{3}{*}{ Mixed/Other } & \multirow[t]{2}{*}{ WAI } & $\begin{array}{l}\text { General } \\
\text { Health }\end{array}$ & 1449 & .281 \\
\hline & & & Age & 1449 & -.147 \\
\hline & & PWA** & Age & 1449 & -.142 \\
\hline $\begin{array}{l}\text { Camerino, D., Conway, P. } \\
\text { M., Estryn-Béhar, M., } \\
\text { Costa, G., \& Hasselhorn, } \\
\text { H. M. (2008). Age- } \\
\text { dependent relationships } \\
\text { between work ability, } \\
\text { thinking of quitting the job, } \\
\text { and actual leaving among } \\
\text { Italian nurses: a } \\
\text { longitudinal study. } \\
\text { International Journal of } \\
\text { Nursing Studies, 45(11), } \\
\text { 1645-1659. }\end{array}$ & $\begin{array}{l}\text { Nurses/Healt } \\
\text { hcare }\end{array}$ & WAI & Age & 5,422 & -.060 \\
\hline
\end{tabular}




\begin{tabular}{|c|c|c|c|c|c|}
\hline \multirow{4}{*}{$\begin{array}{l}\text { Camerino, D., Conway, P. } \\
\text { M., Sartori, S., Campanini, } \\
\text { P., Estryn-Béhar, M., van } \\
\text { der Heijden, B. I. J. M., \& } \\
\text { Costa, G. (2008). Factors } \\
\text { affecting work ability in } \\
\text { day and shift-working } \\
\text { nurses. Chronobiology } \\
\text { international, 25(2-3), 425- } \\
\text { 442. (NEXT Study) }\end{array}$} & \multirow{4}{*}{$\begin{array}{l}\text { Nurses/Healt } \\
\text { hcare }\end{array}$} & \multirow[t]{4}{*}{ WAI } & Reward & 7,332 & .280 \\
\hline & & & Sleep & 7,414 & .330 \\
\hline & & & $\begin{array}{l}\text { Work } \\
\text { Motivation }\end{array}$ & 7,425 & .230 \\
\hline & & & $\begin{array}{l}\text { Job } \\
\text { Satisfaction }\end{array}$ & 7,136 & .195 \\
\hline \multirow{10}{*}{$\begin{array}{l}\text { Camerino, D., Conway, P. } \\
\text { M., Van der Heijden, B. I. } \\
\text { J., Estryn-Behar, M., } \\
\text { Consonni, D., Gould, D., \& } \\
\text { Hasselhorn, H. M. (2006). } \\
\text { Low-perceived work } \\
\text { ability, ageing and intention } \\
\text { to leave nursing: a } \\
\text { comparison among } 10 \\
\text { European } \\
\text { countries. Journal of } \\
\text { Advanced Nursing, 56(5), } \\
\text { 542-552. (NEXT study) }\end{array}$} & $\begin{array}{l}\text { Nurses/Healt } \\
\text { hcare }\end{array}$ & WAI & $\begin{array}{l}\text { Turnover } \\
\text { Intentions }\end{array}$ & 3,121 & -.527 \\
\hline & $\begin{array}{l}\text { Nurses/Healt } \\
\text { hcare }\end{array}$ & WAI & $\begin{array}{l}\text { Turnover } \\
\text { Intentions }\end{array}$ & 2,565 & -.377 \\
\hline & $\begin{array}{l}\text { Nurses/Healt } \\
\text { hcare }\end{array}$ & WAI & $\begin{array}{l}\text { Turnover } \\
\text { Intentions }\end{array}$ & 1,820 & -.251 \\
\hline & $\begin{array}{l}\text { Nurses/Healt } \\
\text { hcare }\end{array}$ & WAI & $\begin{array}{l}\text { Turnover } \\
\text { Intentions }\end{array}$ & 2,105 & -.360 \\
\hline & $\begin{array}{l}\text { Nurses/Healt } \\
\text { hcare }\end{array}$ & WAI & $\begin{array}{l}\text { Turnover } \\
\text { Intentions }\end{array}$ & 1,780 & -.419 \\
\hline & $\begin{array}{l}\text { Nurses/Healt } \\
\text { hcare }\end{array}$ & WAI & $\begin{array}{l}\text { Turnover } \\
\text { Intentions }\end{array}$ & 3,705 & -.290 \\
\hline & $\begin{array}{l}\text { Nurses/Healt } \\
\text { hcare }\end{array}$ & WAI & $\begin{array}{l}\text { Turnover } \\
\text { Intentions }\end{array}$ & 1,301 & -.227 \\
\hline & $\begin{array}{l}\text { Nurses/Healt } \\
\text { hcare }\end{array}$ & WAI & $\begin{array}{l}\text { Turnover } \\
\text { Intentions }\end{array}$ & 3,205 & -.284 \\
\hline & $\begin{array}{l}\text { Nurses/Healt } \\
\text { hcare }\end{array}$ & WAI & $\begin{array}{l}\text { Turnover } \\
\text { Intentions }\end{array}$ & 3,017 & -.210 \\
\hline & $\begin{array}{l}\text { Nurses/Healt } \\
\text { hcare }\end{array}$ & WAI & $\begin{array}{l}\text { Turnover } \\
\text { Intentions }\end{array}$ & 1,876 & -.137 \\
\hline $\begin{array}{l}\text { Carel, R. S., Zusman, M., } \\
\text { \& Karakis, I. (2013). Work }\end{array}$ & $\begin{array}{l}\text { Nurses/Healt } \\
\text { hcare }\end{array}$ & WAI & $\begin{array}{l}\text { Current } \\
\text { Health Status }\end{array}$ & 515 & .306 \\
\hline
\end{tabular}




\begin{tabular}{|c|c|c|c|c|c|}
\hline \multirow{3}{*}{$\begin{array}{l}\text { Ability Index in Israeli } \\
\text { Hospital Nurses: } \\
\text { Applicability of the } \\
\text { Adapted } \\
\text { Questionnaire. Experiment } \\
\text { al Aging Research, 39(5), } \\
\text { 579-590. }\end{array}$} & & & $\begin{array}{l}\text { Pain/Muscul } \\
\text { oskeletal } \\
\text { Symptoms }\end{array}$ & 515 & -.289 \\
\hline & & & Smoking & 505 & -.017 \\
\hline & & & Age & 515 & -.170 \\
\hline \multirow{2}{*}{$\begin{array}{l}\text { Chang, Y., ChenSea, M., } \\
\text { Jang, Y., Wang, J. D. } \\
\text { (2000). A simple self-rating } \\
\text { assessment method of } \\
\text { residual work capability for } \\
\text { occupational permanent } \\
\text { disabilities. American } \\
\text { Journal of Industrial } \\
\text { Medicine, 38, 539-547. }\end{array}$} & Mixed/Other & WAI & $\begin{array}{l}\text { Cognitive/M } \\
\text { ental Ability }\end{array}$ & 152 & .390 \\
\hline & & & $\begin{array}{l}\text { Mental } \\
\text { health }\end{array}$ & 167 & .230 \\
\hline \multirow{4}{*}{$\begin{array}{l}\text { Chiu, M. C., Wang, M. J. } \\
\text { J., Lu, C. W., Pan, S. M., } \\
\text { Kumashiro, M., \& } \\
\text { Ilmarinen, J. (2007). } \\
\text { Evaluating work ability and } \\
\text { quality of life for clinical } \\
\text { nurses in Taiwan. Nursing } \\
\text { Outlook, 55(6), 318-326. }\end{array}$} & $\begin{array}{l}\text { Nurses/Healt } \\
\text { hcare }\end{array}$ & WAI & $\begin{array}{l}\text { Social } \\
\text { Support } \\
\text { (non-work) }\end{array}$ & 1,534 & .355 \\
\hline & & & $\begin{array}{l}\text { Mental } \\
\text { Health }\end{array}$ & 1534 & .370 \\
\hline & & & $\begin{array}{l}\text { Physical } \\
\text { Health }\end{array}$ & 1534 & .390 \\
\hline & & & Age & 1367 & .036 \\
\hline \multirow{2}{*}{$\begin{array}{l}\text { Chung, J., Park, J., Cho, } \\
\text { M., Park, Y., Kim, D., } \\
\text { Yang, D., \& Yang, Y. } \\
\text { (2015). A study on the } \\
\text { relationships between age, } \\
\text { work experience, cognition, } \\
\text { and work ability in older } \\
\text { employees working in } \\
\text { heavy industry. Journal of } \\
\text { Physical Therapy Science, } \\
27(1), 155 .\end{array}$} & Blue Collar & WAI & $\begin{array}{l}\text { Cognitive/M } \\
\text { ental Ability }\end{array}$ & 100 & .210 \\
\hline & & & Age & 100 & . 090 \\
\hline
\end{tabular}




\begin{tabular}{|c|c|c|c|c|c|}
\hline \multirow{12}{*}{$\begin{array}{l}\text { Claudi, J. A. (2012). } \\
\text { Towards a parsimonious } \\
\text { program theory of return to } \\
\text { work intervention. Work, } \\
44(2), 155-164 \text {. }\end{array}$} & \multirow[t]{12}{*}{ Blue Collar } & \multirow[t]{12}{*}{ WAI } & $\begin{array}{l}\text { Coworker } \\
\text { Support }\end{array}$ & 118 & .358 \\
\hline & & & $\begin{array}{l}\text { Justice } \\
\text { Perceptions }\end{array}$ & 118 & .138 \\
\hline & & & $\begin{array}{l}\text { Core Self } \\
\text { Evaluations }\end{array}$ & 118 & .322 \\
\hline & & & Coping & 118 & .147 \\
\hline & & & $\begin{array}{l}\text { Negative } \\
\text { Traits/Emoti } \\
\text { ons }\end{array}$ & 118 & .114 \\
\hline & & & $\begin{array}{l}\text { Social } \\
\text { Support } \\
\text { (non-work) }\end{array}$ & 118 & .315 \\
\hline & & & $\begin{array}{l}\text { Mental } \\
\text { Health }\end{array}$ & 118 & .352 \\
\hline & & & $\begin{array}{l}\text { Physical } \\
\text { Health }\end{array}$ & 118 & .387 \\
\hline & & & Exercise & 118 & .208 \\
\hline & & & $\begin{array}{l}\text { Perceived } \\
\text { Stress }\end{array}$ & 118 & -.499 \\
\hline & & & Age & 118 & .089 \\
\hline & & & $\begin{array}{l}\text { Over } \\
\text { Commitment }\end{array}$ & 118 & -.086 \\
\hline \multirow{3}{*}{$\begin{array}{l}\text { Conway, P. M., Campanini, } \\
\text { P., Sartori, S., Dotti, R., \& } \\
\text { Costa, G. (2008). Main and } \\
\text { interactive effects of } \\
\text { shiftwork, age and work } \\
\text { stress on health in an Italian } \\
\text { sample of healthcare } \\
\text { workers. Applied } \\
\text { Ergonomics, 39(5), 630- } \\
639 .\end{array}$} & \multirow[t]{3}{*}{$\begin{array}{l}\text { Nurses/Healt } \\
\text { hcare }\end{array}$} & \multirow[t]{3}{*}{ WAI } & $\begin{array}{l}\text { Justice } \\
\text { Perceptions }\end{array}$ & 576 & .245 \\
\hline & & & Age & 576 & -.082 \\
\hline & & & $\begin{array}{l}\text { Over } \\
\text { Commitment }\end{array}$ & 576 & -.138 \\
\hline
\end{tabular}




\begin{tabular}{|c|c|c|c|c|c|}
\hline \multirow[t]{9}{*}{$\begin{array}{l}\text { Coomer et al. } 2017- \\
\text { Unpublished }\end{array}$} & \multirow[t]{9}{*}{ Blue Collar } & \multirow[t]{9}{*}{ WAI } & $\begin{array}{l}\text { Core Self } \\
\text { Evaluations }\end{array}$ & 311 & .490 \\
\hline & & & $\begin{array}{l}\text { Conscientiou } \\
\text { sness }\end{array}$ & 311 & .200 \\
\hline & & & $\begin{array}{l}\text { Negative } \\
\text { Traits/Emoti } \\
\text { ons }\end{array}$ & 311 & -.310 \\
\hline & & & $\begin{array}{l}\text { Personal } \\
\text { Resources } \\
\text { (Other) }\end{array}$ & 311 & .088 \\
\hline & & & $\begin{array}{l}\text { Mental } \\
\text { Health }\end{array}$ & 311 & .490 \\
\hline & & & Age & 311 & -.020 \\
\hline & & & $\begin{array}{l}\text { Work } \\
\text { Motivation }\end{array}$ & 311 & .420 \\
\hline & & & $\begin{array}{l}\text { Perceived } \\
\text { Stress }\end{array}$ & 311 & -.340 \\
\hline & & & $\begin{array}{l}\text { Job } \\
\text { Satisfaction }\end{array}$ & 311 & .320 \\
\hline $\begin{array}{l}\text { Costa, A. F., Puga-Leal, R., } \\
\& \text { Nunes, I. L. (2011). An } \\
\text { exploratory study of the } \\
\text { Work Ability Index (WAI) } \\
\text { and its components in a } \\
\text { group of computer workers. } \\
\text { Work, 39(4), 357-367. }\end{array}$ & White Collar & PWA & Age & 50 & -.352 \\
\hline $\begin{array}{l}\text { Costa, G., \& Sartori, S. } \\
\text { (2007). Ageing, working } \\
\text { hours and work ability. } \\
\text { Ergonomics, 50(11), 1914- } \\
1930 .\end{array}$ & Mixed/Other & WAI & Age & 429 & -.191 \\
\hline $\begin{array}{l}\text { D'amato, A., \& Zijlstra, F. } \\
\text { (2010). Toward a climate } \\
\text { for work resumption: the } \\
\text { nonmedical determinants of }\end{array}$ & Mixed/Other & PWA & $\begin{array}{l}\text { General } \\
\text { Health }\end{array}$ & 708 & .214 \\
\hline
\end{tabular}




\begin{tabular}{|c|c|c|c|c|c|}
\hline $\begin{array}{l}\text { return to work. Journal of } \\
\text { Occupational and } \\
\text { Environmental } \\
\text { Medicine, 52(1), 67-80. }\end{array}$ & & & & & \\
\hline \multirow{2}{*}{$\begin{array}{l}\text { da Silva, F. J., Felli, V. E. } \\
\text { A., Martinez, M. C., } \\
\text { Mininel, V. A., \& Ratier, } \\
\text { A. P. P. (2016). Association } \\
\text { between work ability and } \\
\text { fatigue in brazilian nursing } \\
\text { workers. Work: Journal of } \\
\text { Prevention, Assessment \& } \\
\text { Rehabilitation, 53(1), 225- } \\
\text { 232. }\end{array}$} & \multirow[t]{2}{*}{$\begin{array}{l}\text { Nurses/Healt } \\
\text { hcare }\end{array}$} & \multirow[t]{2}{*}{ WAI } & Fatigue & 100 & -.462 \\
\hline & & & Age & 100 & -.099 \\
\hline $\begin{array}{l}\text { de Boer, A. G. E. M., } \\
\text { Bruinvels, D. J., Tytgat, K. } \\
\text { M. A. J., Schoorlemmer, } \\
\text { A., Klinkenbijl, J. H. G., \& } \\
\text { Frings-Dresen, M. H. W. } \\
\text { (2011). Employment status } \\
\text { and work-related problems } \\
\text { of gastrointestinal cancer } \\
\text { patients at diagnosis: a } \\
\text { cross-sectional study. BMJ } \\
\text { Open, } 1(2) \text {, e000190. }\end{array}$ & Mixed/Other & PWA* & $\begin{array}{l}\text { Current } \\
\text { Health Status }\end{array}$ & 45 & .526 \\
\hline \multirow{5}{*}{$\begin{array}{l}\text { De Croon, E. M., Sluiter, J. } \\
\text { K., Nijssen, T. F., } \\
\text { Kammeijer, M., Dijkmans, } \\
\text { B. A. C., Lankhorst, G. J., } \\
\text { \& Frings-Dresen, M. H. W. } \\
\text { (2005). Work ability of } \\
\text { Dutch employees with } \\
\text { rheumatoid } \\
\text { arthritis. Scandinavian } \\
\text { Journal of } \\
\text { Rheumatology, 34(4), 277- } \\
\text { 283. }\end{array}$} & \multirow[t]{5}{*}{ Mixed/Other } & \multirow[t]{5}{*}{ PWA* } & $\begin{array}{l}\text { Physical } \\
\text { Demands }\end{array}$ & 77 & -.539 \\
\hline & & & $\begin{array}{l}\text { Quantitative } \\
\text { Job Demands }\end{array}$ & 76 & -.142 \\
\hline & & & Job Control & 72 & .291 \\
\hline & & & $\begin{array}{l}\text { Coworker } \\
\text { Support }\end{array}$ & 72 & .443 \\
\hline & & & Fatigue & 78 & -.659 \\
\hline
\end{tabular}




\begin{tabular}{|c|c|c|c|c|c|}
\hline \multirow{2}{*}{$\begin{array}{l}\text { de Souza Magnago, T. S. } \\
\text { B., de Lima, A. C. S., } \\
\text { Prochnow, A., da Silva } \\
\text { Ceron, M. D., Tavares, J. } \\
\text { P., \& de Souza Urbanetto, } \\
\text { J. (2012). Intensity of } \\
\text { musculoskeletal pain and } \\
\text { (in) ability to work in } \\
\text { nursing1. Rev. Latino-Am. } \\
\text { Enfermagem, 20(6), 1125- } \\
33 .\end{array}$} & \multirow[t]{2}{*}{$\begin{array}{l}\text { Nurses/Healt } \\
\text { hcare }\end{array}$} & \multirow[t]{2}{*}{ WAI } & $\begin{array}{l}\text { Pain/Muscul } \\
\text { oskeletal } \\
\text { Symptoms }\end{array}$ & 498 & .266 \\
\hline & & & Age & 498 & -.220 \\
\hline $\begin{array}{l}\text { de Vries, H. J., Reneman, } \\
\text { M. F., Groothoff, J. W., } \\
\text { Geertzen, J. H., \& } \\
\text { Brouwer, S. (2013). Self- } \\
\text { reported work ability and } \\
\text { work performance in } \\
\text { workers with chronic } \\
\text { nonspecific } \\
\text { musculoskeletal } \\
\text { pain. Journal of } \\
\text { Occupational } \\
\text { Rehabilitation,23(1), 1-10. }\end{array}$ & Mixed/Other & PWA & Age & 119 & -.029 \\
\hline \multirow{6}{*}{$\begin{array}{l}\text { Deller et al. (2017) } \\
\text { Unpublished }\end{array}$} & \multirow{6}{*}{$\begin{array}{l}\text { White Collar } \\
\text { (Sample 1) }\end{array}$} & \multirow[t]{6}{*}{ WAI } & Job Control & 1093 & .330 \\
\hline & & & $\begin{array}{l}\text { Supervisor } \\
\text { Support }\end{array}$ & 1093 & .297 \\
\hline & & & $\begin{array}{l}\text { Task } \\
\text { Resources }\end{array}$ & 1093 & .230 \\
\hline & & & $\begin{array}{l}\text { Social } \\
\text { Support } \\
\text { (non-work) }\end{array}$ & 1093 & .190 \\
\hline & & & $\begin{array}{l}\text { Mental } \\
\text { Health }\end{array}$ & 1093 & .640 \\
\hline & & & $\begin{array}{l}\text { Physical } \\
\text { Health }\end{array}$ & 1093 & .830 \\
\hline
\end{tabular}




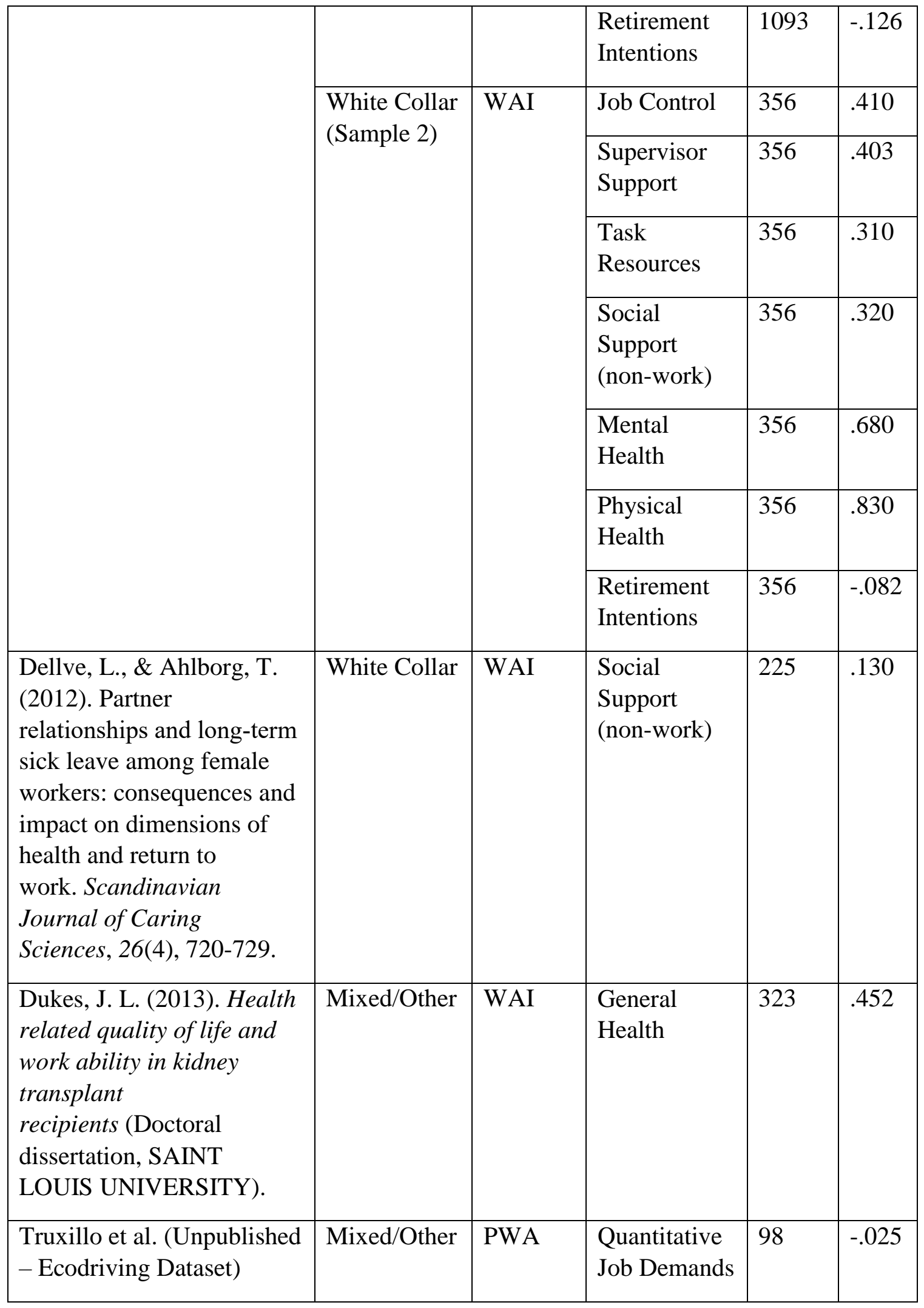




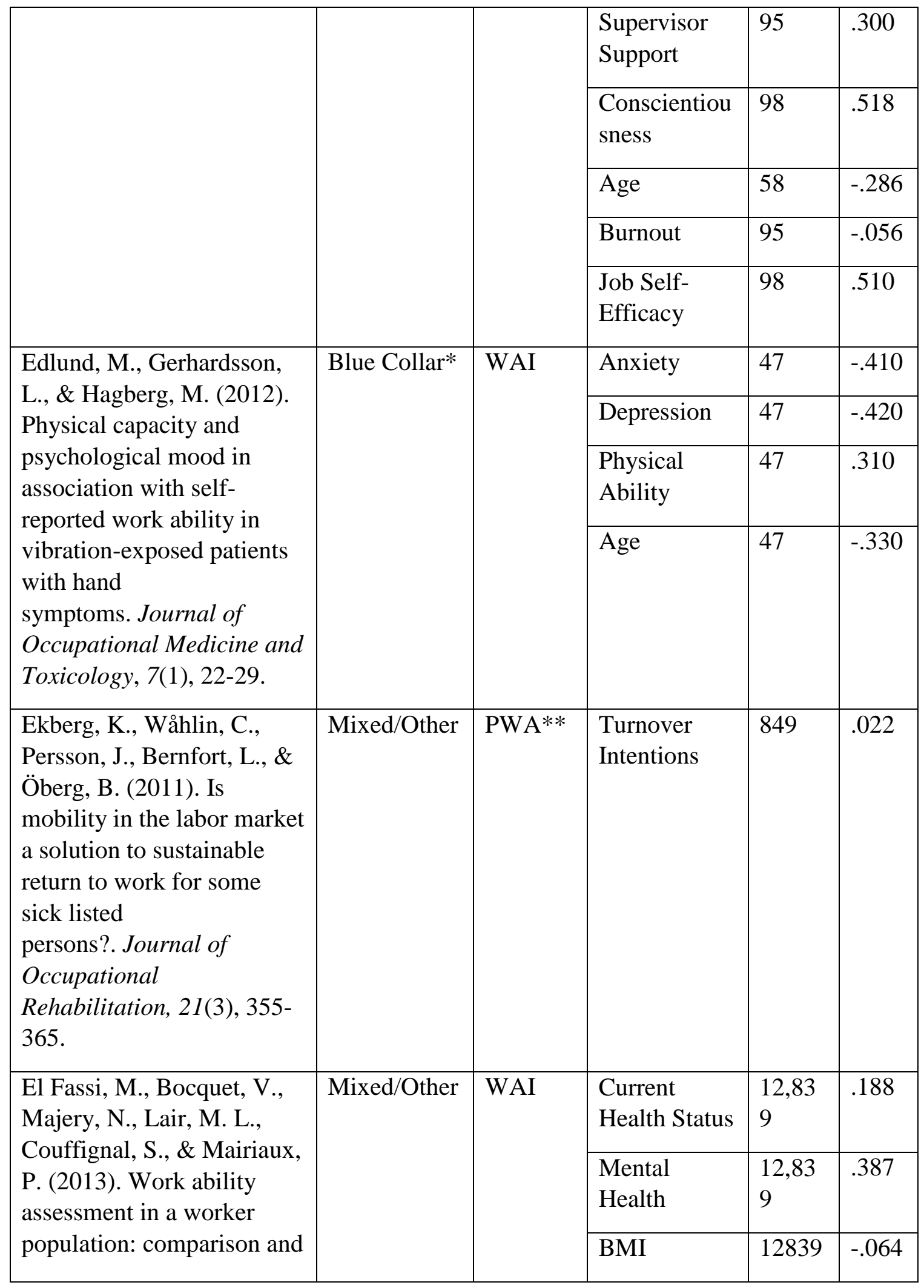




\begin{tabular}{|c|c|c|c|c|c|}
\hline \multirow{9}{*}{$\begin{array}{l}\text { determinants of Work } \\
\text { Ability Index and Work } \\
\text { Ability score. BMC public } \\
\text { health, } 13(1), 305 .\end{array}$} & & & $\begin{array}{l}\text { Pain/Muscul } \\
\text { oskeletal } \\
\text { Symptoms }\end{array}$ & 12839 & -.360 \\
\hline & & & Smoking & 12839 & -.057 \\
\hline & & & Age & 12839 & -.187 \\
\hline & Mixed/Other & PWA** & $\begin{array}{l}\text { Current } \\
\text { Health Status }\end{array}$ & 12839 & .268 \\
\hline & & & $\begin{array}{l}\text { Mental } \\
\text { Health }\end{array}$ & 12839 & .310 \\
\hline & & & $\begin{array}{l}\text { Pain/Muscul } \\
\text { oskeletal } \\
\text { Symptoms }\end{array}$ & 12839 & -.281 \\
\hline & & & BMI & 12839 & -.057 \\
\hline & & & Smoking & 12839 & -.039 \\
\hline & & & Age & 12839 & -.192 \\
\hline \multirow{9}{*}{$\begin{array}{l}\text { Elo, A. L., Ervasti, J., } \\
\text { Kuosma, E., \& Mattila, P. } \\
\text { (2008). Evaluation of an } \\
\text { organizational stress } \\
\text { management program in a } \\
\text { municipal public works } \\
\text { organization. Journal of } \\
\text { Occupational Health } \\
\text { Psychology, 13(1), 10-23. }\end{array}$} & \multirow[t]{9}{*}{ Blue Collar } & \multirow[t]{9}{*}{ WAI } & $\begin{array}{l}\text { Quantitative } \\
\text { Job Demands }\end{array}$ & 625 & -.140 \\
\hline & & & Job Control & 625 & .240 \\
\hline & & & $\begin{array}{l}\text { Supervisor } \\
\text { Support }\end{array}$ & 625 & .220 \\
\hline & & & $\begin{array}{l}\text { Task } \\
\text { Resources }\end{array}$ & 625 & .160 \\
\hline & & & $\begin{array}{l}\text { Justice } \\
\text { Perceptions }\end{array}$ & 625 & .310 \\
\hline & & & $\begin{array}{l}\text { Organization } \\
\text { al Climate }\end{array}$ & 625 & .300 \\
\hline & & & Age & 625 & -.300 \\
\hline & & & Burnout & 625 & -.540 \\
\hline & & & $\begin{array}{l}\text { Perceived } \\
\text { Stress }\end{array}$ & 625 & -.490 \\
\hline
\end{tabular}




\begin{tabular}{|c|c|c|c|c|c|}
\hline $\begin{array}{l}\text { Elovainio, M., Kuusio, H., } \\
\text { Aalto, A. M., Sinervo, T., } \\
\& \text { Heponiemi, T. (2010). } \\
\text { Insecurity and shiftwork as } \\
\text { characteristics of negative } \\
\text { work environment: } \\
\text { psychosocial and } \\
\text { behavioural } \\
\text { mediators. Journal of } \\
\text { Advanced Nursing, 66(5), } \\
\text { 1080-1091. }\end{array}$ & $\begin{array}{l}\text { Nurses/Healt } \\
\text { hcare }\end{array}$ & PWA** & $\begin{array}{l}\text { Justice } \\
\text { Perceptions }\end{array}$ & 2092 & .020 \\
\hline \multirow{2}{*}{$\begin{array}{l}\text { Emmerich, A. I., \& Rigotti, } \\
\text { T. (2017). Reciprocal } \\
\text { relations between work- } \\
\text { related authenticity and } \\
\text { intrinsic motivation, work } \\
\text { ability and depressivity: A } \\
\text { two-wave study. Frontiers } \\
\text { in Psychology, 8, 1-12. }\end{array}$} & \multirow[t]{2}{*}{ White Collar } & \multirow[t]{2}{*}{ PWA* } & Depression & 780 & -.529 \\
\hline & & & $\begin{array}{l}\text { Work } \\
\text { Motivation }\end{array}$ & 780 & .365 \\
\hline \multirow{5}{*}{$\begin{array}{l}\text { Feldt, T., Hyvönen, K., } \\
\text { Mäkikangas, A., Kinnunen, } \\
\text { U., \& Kokko, K. (2009). } \\
\text { Development trajectories of } \\
\text { Finnish managers' work } \\
\text { ability over a 10-year } \\
\text { follow-up period. } \\
\text { Scandinavian journal of } \\
\text { work, environment \& } \\
\text { health, 37-47.† }\end{array}$} & \multirow[t]{5}{*}{ White Collar } & \multirow[t]{5}{*}{ WAI } & Job Control & 818 & .210 \\
\hline & & & $\begin{array}{l}\text { Organization } \\
\text { al Climate }\end{array}$ & 818 & .190 \\
\hline & & & Age & 818 & -.217 \\
\hline & & & Motivation & 785 & -.02 \\
\hline & & & $\begin{array}{l}\text { Organization } \\
\text { al } \\
\text { Commitment }\end{array}$ & 818 & .124 \\
\hline \multirow{2}{*}{$\begin{array}{l}\text { Feldt, T., Hyvönen, K., } \\
\text { Oja-Lipasti, T., Kinnunen, } \\
\text { U., \& Salmela-Aro, K. } \\
\text { (2012). Do work ability and } \\
\text { job involvement channel } \\
\text { later personal goals in } \\
\text { retirement? An 11-year } \\
\text { follow-up study. } \\
\text { International Archives of } \\
\text { Occupational and }\end{array}$} & \multirow[t]{2}{*}{ White Collar } & \multirow[t]{2}{*}{ WAI } & $\begin{array}{l}\text { Work } \\
\text { Motivation }\end{array}$ & 120 & .10 \\
\hline & & & Retirement & 120 & -.260 \\
\hline
\end{tabular}




\begin{tabular}{|c|c|c|c|c|c|}
\hline $\begin{array}{l}\text { Environmental Health, } \\
85(5), 547-558 . \dagger\end{array}$ & & & & & \\
\hline \multirow{4}{*}{$\begin{array}{l}\text { Firoozeh, M., Saremi, M., } \\
\text { Kavousi, A., \& Maleki, A. } \\
\text { (2017). Demographic and } \\
\text { occupational determinants } \\
\text { of the work ability of } \\
\text { firemen. Journal of } \\
\text { Occupational } \\
\text { Health, 59(1), 81-87. }\end{array}$} & \multirow[t]{4}{*}{ Mixed/Other } & \multirow[t]{4}{*}{ WAI } & BMI & 351 & -.187 \\
\hline & & & Exercise & 354 & .206 \\
\hline & & & Smoking & 361 & -.116 \\
\hline & & & Age & 354 & -.277 \\
\hline \multirow{8}{*}{$\begin{array}{l}\text { Fischer, F. M., \& Martinez, } \\
\text { M. C. (2011). Work ability } \\
\text { among hospital food } \\
\text { service professionals: } \\
\text { multiple associated } \\
\text { variables require } \\
\text { comprehensive } \\
\text { intervention. Work, 41, } \\
\text { 3746-3752. }\end{array}$} & \multirow[t]{8}{*}{ Blue Collar } & \multirow[t]{8}{*}{ WAI } & $\begin{array}{l}\text { Workplace } \\
\text { Mistreatment }\end{array}$ & 72 & -.169 \\
\hline & & & $\begin{array}{l}\text { Quantitative } \\
\text { Job Demands }\end{array}$ & 75 & .032 \\
\hline & & & Job Control & 72 & .033 \\
\hline & & & $\begin{array}{l}\text { Coworker } \\
\text { Support }\end{array}$ & 74 & .384 \\
\hline & & & $\begin{array}{l}\text { Justice } \\
\text { Perceptions }\end{array}$ & 68 & .502 \\
\hline & & & BMI & 74 & -.038 \\
\hline & & & Age & 76 & .159 \\
\hline & & & $\begin{array}{l}\text { Over } \\
\text { Commitment }\end{array}$ & 75 & -.332 \\
\hline \multirow{6}{*}{$\begin{array}{l}\text { Fischer, F. M., \& Martinez, } \\
\text { M. C. (2013). Individual } \\
\text { features, working } \\
\text { conditions and work } \\
\text { injuries are associated with } \\
\text { work ability among nursing } \\
\text { professionals. Work, 45(4), } \\
\text { 509-517. }\end{array}$} & \multirow[t]{6}{*}{$\begin{array}{l}\text { Nurses/Healt } \\
\text { hcare }\end{array}$} & \multirow[t]{6}{*}{ WAI } & $\begin{array}{l}\text { Workplace } \\
\text { Mistreatment }\end{array}$ & 490 & -.208 \\
\hline & & & $\begin{array}{l}\text { Quantitative } \\
\text { Job Demands }\end{array}$ & 506 & -.271 \\
\hline & & & Job Demands & 505 & -.329 \\
\hline & & & Job Control & 502 & .068 \\
\hline & & & $\begin{array}{l}\text { Coworker } \\
\text { Support }\end{array}$ & 503 & .324 \\
\hline & & & Reward & 505 & .318 \\
\hline
\end{tabular}




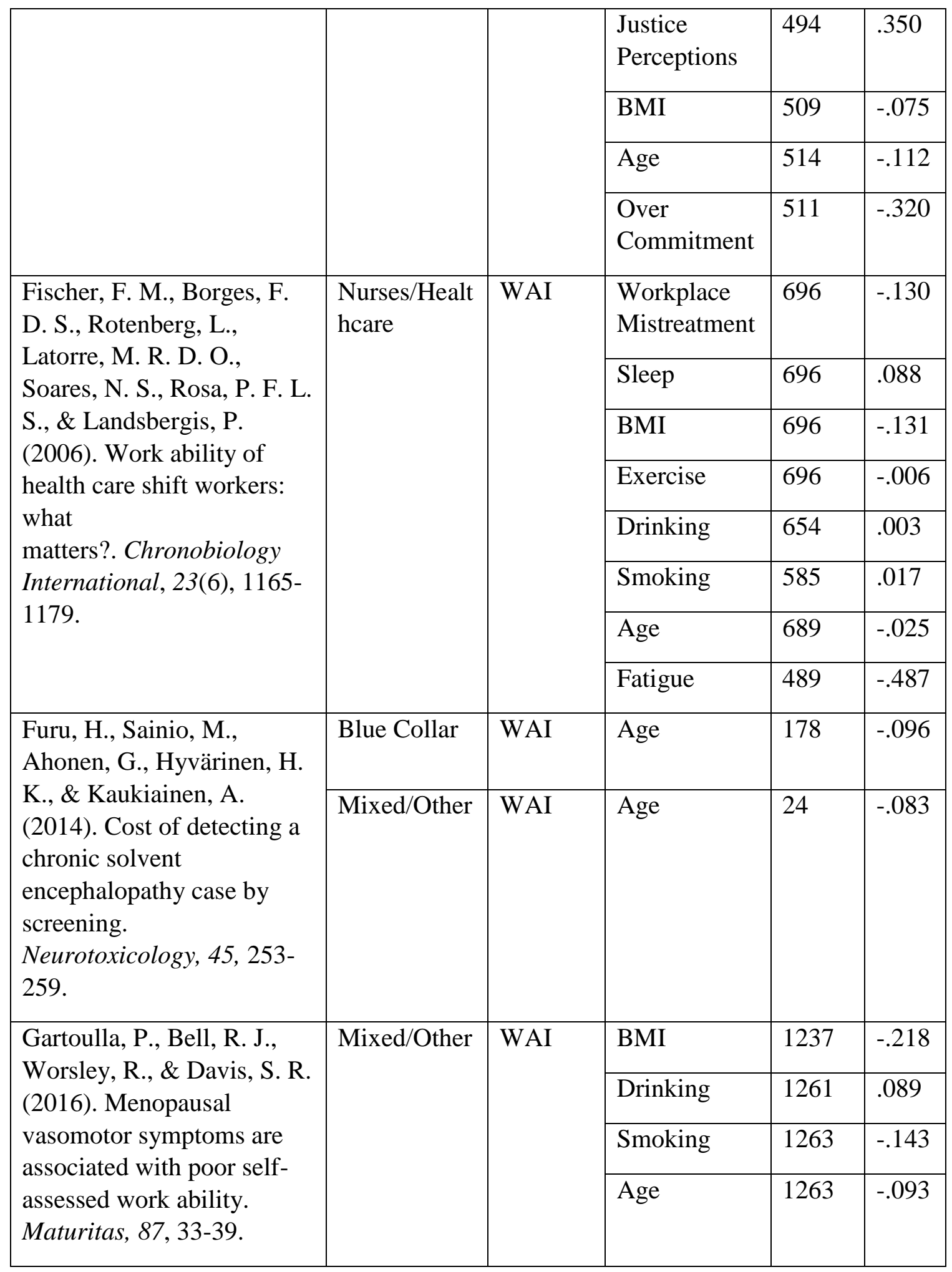




\begin{tabular}{|c|c|c|c|c|c|}
\hline \multirow{4}{*}{$\begin{array}{l}\text { Geukes, M., van Aalst, M. } \\
\text { P., Nauta, M. C., \& } \\
\text { Oosterhof, H. (2012). The } \\
\text { impact of menopausal } \\
\text { symptoms on work ability. } \\
\text { Menopause, 19(3), 278- } \\
\text { 282. }\end{array}$} & \multirow[t]{4}{*}{$\begin{array}{l}\text { Nurses/Healt } \\
\text { hcare }\end{array}$} & \multirow[t]{4}{*}{ WAI } & $\begin{array}{l}\text { Symptoms - } \\
\text { Non Pain } \\
\text { related }\end{array}$ & 211 & -.365 \\
\hline & & & $\begin{array}{l}\text { Mental } \\
\text { Health }\end{array}$ & 197 & .570 \\
\hline & & & Depression & 197 & -.520 \\
\hline & & & Anxiety & 211 & -.530 \\
\hline $\begin{array}{l}\text { Ghaddar, A., Ronda, E., \& } \\
\text { Nolasco, A. (2011). Work } \\
\text { ability, psychosocial } \\
\text { hazards and work } \\
\text { experience in prison } \\
\text { environments. Occupationa } \\
\text { l Medicine, 61, 503-508. }\end{array}$ & Mixed/Other & WAI & Age & 439 & -.242 \\
\hline $\begin{array}{l}\text { Glise, K., Hadzibajramovic, } \\
\text { E., Jonsdottir, I. H., \& } \\
\text { Ahlborg, G. (2010). Self- } \\
\text { reported exhaustion: a } \\
\text { possible indicator of } \\
\text { reduced work ability and } \\
\text { increased risk of sickness } \\
\text { absence among human } \\
\text { service } \\
\text { workers. International } \\
\text { Archives of Occupational } \\
\text { and Environmental } \\
\text { Health, 83(5), 511-520. }\end{array}$ & Mixed/Other & WAI & $\begin{array}{l}\text { General } \\
\text { Health }\end{array}$ & 2010 & .494 \\
\hline \multirow{3}{*}{$\begin{array}{l}\text { Godinho, M. R., Greco, R. } \\
\text { M., Teixeira, M. T. B., } \\
\text { Teixeira, L. R., Guerra, M. } \\
\text { R., \& Chaoubah, A. (2016). } \\
\text { Work ability and associated } \\
\text { factors of Brazilian } \\
\text { technical-administrative } \\
\text { workers in education. BMC } \\
\text { Research Notes, 9(1), 1-10. }\end{array}$} & \multirow[t]{3}{*}{ White Collar } & \multirow[t]{3}{*}{ WAI } & $\begin{array}{l}\text { Social } \\
\text { support } \\
\text { (General at } \\
\text { work) }\end{array}$ & 579 & .238 \\
\hline & & & $\begin{array}{l}\text { Social } \\
\text { Support } \\
\text { (non-work }\end{array}$ & 565 & .190 \\
\hline & & & $\begin{array}{l}\text { General } \\
\text { Health }\end{array}$ & 590 & .300 \\
\hline
\end{tabular}




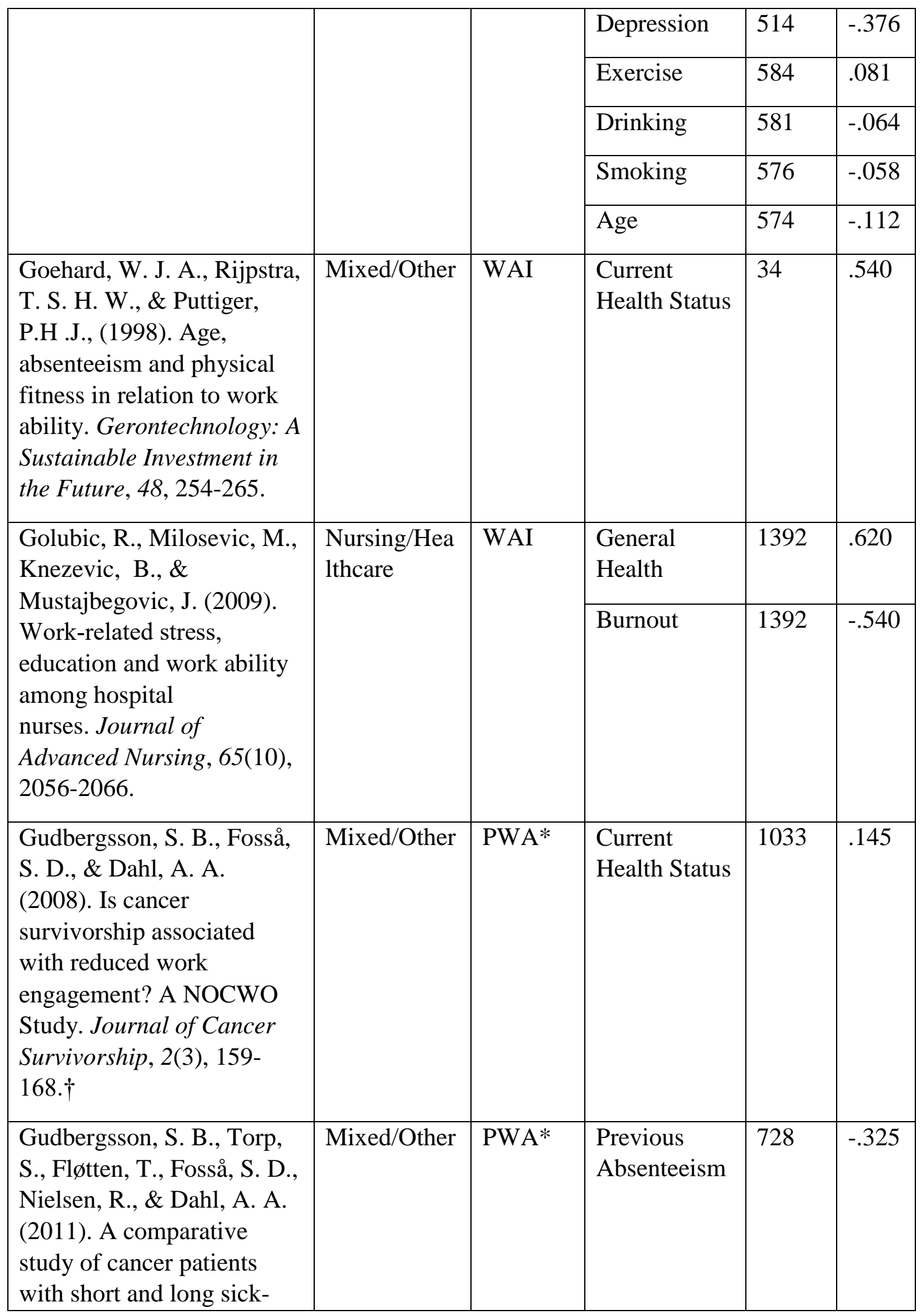




\begin{tabular}{|c|c|c|c|c|c|}
\hline $\begin{array}{l}\text { leave after primary } \\
\text { treatment. Acta } \\
\text { Oncologica, 50(3), 381- } \\
389 . \dagger\end{array}$ & & & & & \\
\hline \multirow{7}{*}{$\begin{array}{l}\text { Guidi, S., Bagnara, S., \& } \\
\text { Fichera, G. P. (2012). The } \\
\text { HSE indicator tool, } \\
\text { psychological distress and } \\
\text { work ability. Occupational } \\
\text { Medicine, 62(3), 203-209. }\end{array}$} & \multirow[t]{7}{*}{ White Collar } & \multirow[t]{7}{*}{ WAI } & $\begin{array}{l}\text { Workplace } \\
\text { Mistreatment }\end{array}$ & 352 & -.290 \\
\hline & & & Job Demands & 352 & -.331 \\
\hline & & & $\begin{array}{l}\text { Mental/Emot } \\
\text { ional } \\
\text { Demands }\end{array}$ & 352 & -.380 \\
\hline & & & Job Control & 352 & .390 \\
\hline & & & $\begin{array}{l}\text { Supervisor } \\
\text { Support }\end{array}$ & 352 & .320 \\
\hline & & & $\begin{array}{l}\text { Coworker } \\
\text { Support }\end{array}$ & 352 & .300 \\
\hline & & & $\begin{array}{l}\text { Mental } \\
\text { Health }\end{array}$ & 352 & .559 \\
\hline \multirow{9}{*}{$\begin{array}{l}\text { Gupta, N., Jensen, B. S., } \\
\text { Søgaard, K., Carneiro, I. } \\
\text { G., Christiansen, C. S., } \\
\text { Hanisch, C., \& } \\
\text { Holtermann, A. (2014). } \\
\text { Face validity of the single } \\
\text { work ability item: } \\
\text { comparison with } \\
\text { objectively measured heart } \\
\text { rate reserve over several } \\
\text { days. International Journal } \\
\text { of Environmental Research } \\
\text { and Public Health, 11(5), } \\
\text { 5333-5348. }\end{array}$} & \multirow{4}{*}{$\begin{array}{l}\text { Blue Collar } \\
\text { (Sample 1) }\end{array}$} & \multirow[t]{4}{*}{ PWA } & Job Control & 53 & .165 \\
\hline & & & BMI & 53 & -.021 \\
\hline & & & $\begin{array}{l}\text { Physical } \\
\text { Ability }\end{array}$ & 53 & -.110 \\
\hline & & & Age & 53 & .005 \\
\hline & \multirow{5}{*}{$\begin{array}{l}\text { Blue Collar } \\
\text { (Sample 2) }\end{array}$} & \multirow[t]{5}{*}{ PWA } & Job Control & 74 & .219 \\
\hline & & & BMI & 74 & .043 \\
\hline & & & $\begin{array}{l}\text { Physical } \\
\text { Ability }\end{array}$ & 74 & .330 \\
\hline & & & Age & 74 & .255 \\
\hline & & & $\begin{array}{l}\text { Smoking } \\
\text { (both } \\
\text { samples) }\end{array}$ & 127 & -.067 \\
\hline
\end{tabular}




\begin{tabular}{|c|c|c|c|c|c|}
\hline $\begin{array}{l}\text { Gustafsson, K., \& } \\
\text { Marklund, S. (2011). } \\
\text { Consequences of sickness } \\
\text { presence and sickness } \\
\text { absence on health and work } \\
\text { ability: a Swedish } \\
\text { prospective cohort } \\
\text { study. International } \\
\text { Journal of Occupational } \\
\text { Medicine and } \\
\text { Environmental Health, } \\
24(2), 153-165 . \dagger\end{array}$ & Mixed/Other & PWA & $\begin{array}{l}\text { Previous } \\
\text { Absenteeism }\end{array}$ & 2181 & -.239 \\
\hline $\begin{array}{l}\text { Gustafsson, K., \& } \\
\text { Marklund, S. (2014). } \\
\text { Associations between } \\
\text { health and combinations of } \\
\text { sickness presence and } \\
\text { absence. Occupational } \\
\text { Medicine, 64(1), 49-55.† }\end{array}$ & Mixed/Other & PWA & $\begin{array}{l}\text { Future } \\
\text { Absenteeism }\end{array}$ & 1727 & -.695 \\
\hline \multirow{4}{*}{$\begin{array}{l}\text { Habibi, E., Dehghan, H., } \\
\text { Safari, S., Mahaki, B., \& } \\
\text { Hassanzadeh, A. (2014). } \\
\text { Effects of work-related } \\
\text { stress on work ability index } \\
\text { among refinery workers. } \\
\text { Journal of Education and } \\
\text { Health Promotion, 3(1), } \\
\text { 18-25. }\end{array}$} & \multirow[t]{4}{*}{ Blue Collar } & \multirow[t]{4}{*}{ WAI } & $\begin{array}{l}\text { Physical } \\
\text { Demands }\end{array}$ & 171 & -.198 \\
\hline & & & $\begin{array}{l}\text { Social } \\
\text { support } \\
\text { (General at } \\
\text { work) }\end{array}$ & 171 & .157 \\
\hline & & & $\begin{array}{l}\text { Perceived } \\
\text { Stress }\end{array}$ & 171 & -.156 \\
\hline & & & $\begin{array}{l}\text { Organization } \\
\text { al } \\
\text { Commitment }\end{array}$ & 171 & .213 \\
\hline $\begin{array}{l}\text { Habibi, E., Dehghan, H., } \\
\text { Zeinodini, M., Yousefi, H., } \\
\text { \& Hasanzadeh, A. (2012). } \\
\text { A study on work ability } \\
\text { index and physical work } \\
\text { capacity on the base of fax } \\
\text { equation VO2 max in male } \\
\text { nursing hospital staff in }\end{array}$ & $\begin{array}{l}\text { Nurses/Healt } \\
\text { hcare }\end{array}$ & WAI & Age & 228 & -.840 \\
\hline
\end{tabular}




\begin{tabular}{|c|c|c|c|c|c|}
\hline $\begin{array}{l}\text { Isfahan, Iran. International } \\
\text { Journal of Preventive } \\
\text { Medicine, } 3(11), 776-782\end{array}$ & & & & & \\
\hline \multirow{15}{*}{$\begin{array}{l}\text { Hakanen, J. J., Bakker, A. } \\
\text { B., \& Schaufeli, W. B. } \\
\text { (2006). Burnout and work } \\
\text { engagement among } \\
\text { teachers. Journal of School } \\
\text { Psychology, 43(6), 495- } \\
\text { 513. }\end{array}$} & \multirow[t]{11}{*}{$\begin{array}{l}\text { White Collar } \\
\text { (Sample 1) }\end{array}$} & \multirow[t]{11}{*}{$\mathrm{PWA}^{* *}$} & $\begin{array}{l}\text { Workplace } \\
\text { Mistreatment }\end{array}$ & 1019 & -.150 \\
\hline & & & $\begin{array}{l}\text { Physical } \\
\text { Demands }\end{array}$ & 1019 & -.200 \\
\hline & & & $\begin{array}{l}\text { Quantitative } \\
\text { Job Demands }\end{array}$ & 1019 & -.150 \\
\hline & & & Job Control & 1019 & .210 \\
\hline & & & $\begin{array}{l}\text { Supervisor } \\
\text { Support }\end{array}$ & 1019 & .150 \\
\hline & & & $\begin{array}{l}\text { Task } \\
\text { Resources }\end{array}$ & 1019 & .190 \\
\hline & & & $\begin{array}{l}\text { Organization } \\
\text { al Climate }\end{array}$ & 1019 & .165 \\
\hline & & & $\begin{array}{l}\text { General } \\
\text { Health }\end{array}$ & 1019 & .360 \\
\hline & & & $\begin{array}{l}\text { Work } \\
\text { Motivation }\end{array}$ & 1019 & .415 \\
\hline & & & Burnout & 1019 & -.465 \\
\hline & & & $\begin{array}{l}\text { Organization } \\
\text { al } \\
\text { Commitment }\end{array}$ & 1019 & .260 \\
\hline & \multirow[t]{4}{*}{$\begin{array}{l}\text { White Collar } \\
\text { (Sample 2) }\end{array}$} & \multirow[t]{4}{*}{ PWA** } & $\begin{array}{l}\text { Workplace } \\
\text { Mistreatment }\end{array}$ & 1019 & -.160 \\
\hline & & & $\begin{array}{l}\text { Physical } \\
\text { Demands }\end{array}$ & 1019 & -.260 \\
\hline & & & $\begin{array}{l}\text { Quantitative } \\
\text { Job Demands }\end{array}$ & 1019 & -.270 \\
\hline & & & Job Control & 1019 & .290 \\
\hline
\end{tabular}




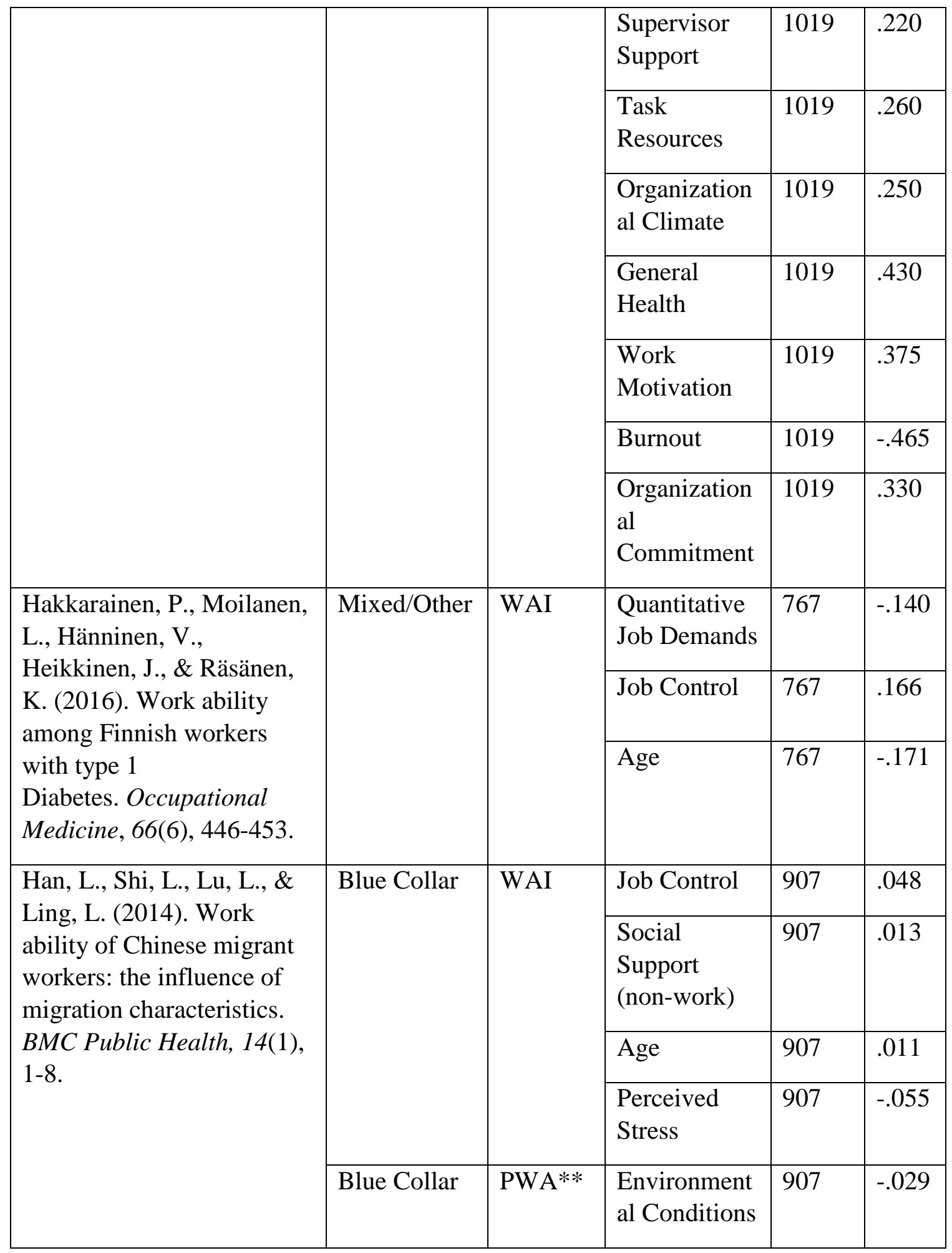




\begin{tabular}{|c|c|c|c|c|c|}
\hline & & & $\begin{array}{l}\text { Social } \\
\text { Support } \\
\text { (non-work) }\end{array}$ & 907 & .011 \\
\hline & & & Job Control & 907 & .043 \\
\hline & & & Age & 907 & .008 \\
\hline & & & $\begin{array}{l}\text { Perceived } \\
\text { Stress }\end{array}$ & 907 & -.023 \\
\hline $\begin{array}{l}\text { Hansen, K. E., Kesmodel, } \\
\text { U. S., Baldursson, E. B., }\end{array}$ & Mixed/Other & WAI & $\begin{array}{l}\text { Previous } \\
\text { Abstenteeism }\end{array}$ & 1070 & -.555 \\
\hline $\begin{array}{l}\text { Schultz, R., \& Forman, A. } \\
\text { (2013). The influence of } \\
\text { endometriosis-related } \\
\text { symptoms on work life and }\end{array}$ & & & $\begin{array}{l}\text { Symptoms - } \\
\text { Non Pain } \\
\text { related }\end{array}$ & 1070 & -.383 \\
\hline work ability: a study of & & & Depression & 1070 & -.637 \\
\hline $\begin{array}{l}\text { patients in employment. } \\
\text { European Journal of } \\
\text { Obstetrics \& Gynecology }\end{array}$ & & & $\begin{array}{l}\text { Pain/Muscul } \\
\text { oskeletal } \\
\text { Symptoms }\end{array}$ & 1070 & -.457 \\
\hline and Reproductive Biology, & & & Age & 1070 & -.017 \\
\hline & & & Fatigue & 1070 & -.530 \\
\hline & & & $\begin{array}{l}\text { Perceived } \\
\text { Stress }\end{array}$ & 1070 & -.490 \\
\hline $\begin{array}{l}\text { Hellemans, C., \& Lapthorn, } \\
\text { B. (2016). Antecedents of }\end{array}$ & Blue Collar & WAI & $\begin{array}{l}\text { Physical } \\
\text { demands }\end{array}$ & 603 & -.270 \\
\hline $\begin{array}{l}\text { sector: From health, } \\
\text { competence and pleasure at }\end{array}$ & & & $\begin{array}{l}\text { Quantitative } \\
\text { Job Demands }\end{array}$ & 603 & -.290 \\
\hline $\begin{array}{l}\text { work to working } \\
\text { conditions. International }\end{array}$ & & & Job control & 603 & .210 \\
\hline $\begin{array}{l}\text { Journal of Workplace } \\
\text { Health Management, 9(3), } \\
\text { 328-339. }\end{array}$ & & & $\begin{array}{l}\text { Job } \\
\text { Resources } \\
\text { (Other) }\end{array}$ & 603 & 200 \\
\hline & & & $\begin{array}{l}\text { General } \\
\text { Health }\end{array}$ & 603 & .470 \\
\hline
\end{tabular}




\begin{tabular}{|c|c|c|c|c|c|}
\hline & & & $\begin{array}{l}\text { Work } \\
\text { Motivation }\end{array}$ & 603 & .430 \\
\hline $\begin{array}{l}\text { Heponiemi, T., Kouvonen, } \\
\text { A., Vänskä, J., Halila, H., } \\
\text { Sinervo, T., Kivimäki, M., } \\
\text { \& Elovainio, M. (2008). } \\
\text { Health, psychosocial } \\
\text { factors and retirement } \\
\text { intentions among Finnish } \\
\text { physicians. Occupational } \\
\text { Medicine, 58(6), 406-412. }\end{array}$ & $\begin{array}{l}\text { Nurses/Healt } \\
\text { hcare }\end{array}$ & PWA** & $\begin{array}{l}\text { Retirement } \\
\text { Intentions }\end{array}$ & 1383 & -.210 \\
\hline $\begin{array}{l}\text { Holm, H. V., Fosså, S. D., } \\
\text { Hedlund, H., \& Dahl, A. A. } \\
\text { (2013). Study of generic } \\
\text { quality of life in patients } \\
\text { operated on for post- } \\
\text { prostatectomy } \\
\text { incontinence. International } \\
\text { Journal of Urology, 20(9), } \\
\text { 889-895. }\end{array}$ & Mixed/Other & $\mathrm{PWA}^{* *}$ & $\begin{array}{l}\text { General } \\
\text { Health }\end{array}$ & 85 & .476 \\
\hline \multirow{2}{*}{$\begin{array}{l}\text { Holtermann, A., Hansen, J. } \\
\text { V., Burr, H., \& Søgaard, K. } \\
\text { (2010). Prognostic factors } \\
\text { for long-term sickness } \\
\text { absence among employees } \\
\text { with neck-shoulder and } \\
\text { low-back pain. } \\
\text { Scandinavian Journal of } \\
\text { Work, Environment \& } \\
\text { Health, 36(1), 34-41. }\end{array}$} & Mixed/Other & PWA* & $\begin{array}{l}\text { Pain/Muscul } \\
\text { oskeletal } \\
\text { Symptoms }\end{array}$ & 848 & -.137 \\
\hline & Mixed/Other & PWA* & $\begin{array}{l}\text { Pain/Muscul } \\
\text { oskeletal } \\
\text { Symptoms }\end{array}$ & 676 & -.121 \\
\hline $\begin{array}{l}\text { Hoonakker, P., \& Van } \\
\text { Duivenbooden, C. (2012). } \\
\text { Predicting long-term } \\
\text { absenteeism from work in } \\
\text { construction industry: a } \\
\text { longitudinal } \\
\text { study. Work, 41(1), 3765- } \\
3770 .\end{array}$ & Mixed/Other & WAI & $\begin{array}{l}\text { Future } \\
\text { Absenteeism }\end{array}$ & 8418 & -.092 \\
\hline
\end{tabular}




\begin{tabular}{|c|c|c|c|c|c|}
\hline \multirow{2}{*}{$\begin{array}{l}\text { Ilmarinen, J., \& Tuomi, K. } \\
\text { (1992). Work ability of } \\
\text { aging } \\
\text { workers. Scandinavian } \\
\text { Journal of Work, } \\
\text { Environment \& Health, 23, } \\
\text { 8-10. (FLAME) } \dagger\end{array}$} & $\begin{array}{l}\text { Mixed/Other } \\
\text { (Sample 1) }\end{array}$ & WAI & $\begin{array}{l}\text { Future } \\
\text { Disability } \\
\text { Status }\end{array}$ & 733 & -.749 \\
\hline & $\begin{array}{l}\text { Mixed/Other } \\
\text { (Sample 2) }\end{array}$ & WAI & $\begin{array}{l}\text { Future } \\
\text { Disability } \\
\text { Status }\end{array}$ & 849 & -.675 \\
\hline \multirow{10}{*}{$\begin{array}{l}\text { Ilmarinen, V., Ilmarinen, J., } \\
\text { Huuhtanen, P., Louhevaara, } \\
\text { V., \& Näsman, O. (2015). } \\
\text { Examining the factorial } \\
\text { structure, measurement } \\
\text { invariance and convergent } \\
\text { and discriminant validity of } \\
\text { a novel self-report measure } \\
\text { of work ability: work } \\
\text { ability-personal radar. } \\
\text { Ergonomics, 58(8), 1445- } \\
\text { 1460. }\end{array}$} & \multirow[t]{10}{*}{ Mixed/Other } & \multirow[t]{10}{*}{ PWA** } & Job Control & 3,754 & .310 \\
\hline & & & $\begin{array}{l}\text { Supervisor } \\
\text { Support }\end{array}$ & 3,754 & .350 \\
\hline & & & $\begin{array}{l}\text { Coworker } \\
\text { Support }\end{array}$ & 3,754 & .300 \\
\hline & & & $\begin{array}{l}\text { Justice } \\
\text { Perceptions }\end{array}$ & 3,754 & .380 \\
\hline & & & $\begin{array}{l}\text { Task } \\
\text { Resources }\end{array}$ & 3,754 & .290 \\
\hline & & & Reward & 3,754 & .370 \\
\hline & & & $\begin{array}{l}\text { General } \\
\text { Health }\end{array}$ & 3,754 & .680 \\
\hline & & & Age & 3,754 & -.140 \\
\hline & & & $\begin{array}{l}\text { Organization } \\
\text { al } \\
\text { Commitment }\end{array}$ & 3,754 & .400 \\
\hline & & & $\begin{array}{l}\text { Work } \\
\text { Motivation }\end{array}$ & 3,754 & .460 \\
\hline \multirow{5}{*}{$\begin{array}{l}\text { Ję dryka-Góral, A., } \\
\text { Bugajska, J., Łastowiecka, } \\
\text { E., Najmiec, A., Rell- } \\
\text { Bakalarska, M., Bownik, I., } \\
\text { \& Kochmański, M. (2006). } \\
\text { Work ability in ageing } \\
\text { workers suffering from } \\
\text { chronic diseases. } \\
\text { International Journal of }\end{array}$} & \multirow[t]{2}{*}{ Mixed/Other } & \multirow[t]{2}{*}{ WAI } & Job Demands & 191 & -.104 \\
\hline & & & Age & 265 & -.104 \\
\hline & Mixed/Other & WAI & Job Demands & 65 & .068 \\
\hline & \multirow[t]{2}{*}{ Mixed/Other } & \multirow[t]{2}{*}{ WAI } & Job Demands & 112 & -.137 \\
\hline & & & Age & 135 & .124 \\
\hline
\end{tabular}




\begin{tabular}{|c|c|c|c|c|c|}
\hline $\begin{array}{l}\text { Occupational Safety and } \\
\text { Ergonomics, } 12(1), 17-30 .\end{array}$ & & & & & \\
\hline \multirow{8}{*}{$\begin{array}{l}\text { Kabat-Farr, D., Walsh, B. } \\
\text { M., \& McGonagle, A. K. } \\
\text { (2017). Uncivil supervisors } \\
\text { and perceived work ability: } \\
\text { The joint moderating roles } \\
\text { of job involvement and } \\
\text { grit. Journal of Business } \\
\text { Ethics, doi:http://dx.doi.org } \\
\text {.proxy.lib.pdx.edu/10.1007/ } \\
\text { s10551-017-3604-5 }\end{array}$} & \multirow[t]{4}{*}{ Mixed/Other } & \multirow[t]{4}{*}{ PWA } & $\begin{array}{l}\text { Workplace } \\
\text { Mistreatment }\end{array}$ & 399 & -.285 \\
\hline & & & Grit & 399 & .360 \\
\hline & & & $\begin{array}{l}\text { Negative } \\
\text { Traits/Emoti } \\
\text { ons }\end{array}$ & 399 & .370 \\
\hline & & & $\begin{array}{l}\text { Work } \\
\text { Motivation }\end{array}$ & 399 & -.020 \\
\hline & \multirow[t]{4}{*}{$\begin{array}{l}\text { Mentally } \\
\text { Demanding }\end{array}$} & \multirow[t]{4}{*}{ PWA } & $\begin{array}{l}\text { Workplace } \\
\text { Mistreatment }\end{array}$ & 116 & -.360 \\
\hline & & & Grit & 116 & .400 \\
\hline & & & $\begin{array}{l}\text { Negative } \\
\text { Traits/Emoti } \\
\text { ons }\end{array}$ & 116 & .370 \\
\hline & & & $\begin{array}{l}\text { Work } \\
\text { Motivation }\end{array}$ & 116 & -.020 \\
\hline \multirow{5}{*}{$\begin{array}{l}\text { Kaewboonchoo, O., \& } \\
\text { Ratanasiripong, P. (2015). } \\
\text { Psychometric properties of } \\
\text { the Thai version of the } \\
\text { work ability index (Thai } \\
\text { WAI). Journal of } \\
\text { Occupational Health, } \\
57(4), 371-377 .\end{array}$} & \multirow[t]{5}{*}{ Mixed/Other } & \multirow[t]{5}{*}{ WAI } & $\begin{array}{l}\text { Current } \\
\text { Health Status }\end{array}$ & 2744 & .570 \\
\hline & & & $\begin{array}{l}\text { Symptoms - } \\
\text { Non Pain } \\
\text { related }\end{array}$ & 2744 & -.315 \\
\hline & & & $\begin{array}{l}\text { Mental } \\
\text { Health }\end{array}$ & 2744 & .199 \\
\hline & & & Depression & 2744 & -.274 \\
\hline & & & Anxiety & 2744 & -.314 \\
\hline \multirow{2}{*}{$\begin{array}{l}\text { Kaewboonchoo, O., Isahak, } \\
\text { M., Susilowati, I., Phuong, } \\
\text { T. N., Morioka, I., } \\
\text { Harncharoen, K., \& } \\
\text { Ratanasiripong, P. (2016). }\end{array}$} & \multirow[t]{2}{*}{$\begin{array}{l}\text { Mixed/Other } \\
\text { (Sample 1) }\end{array}$} & \multirow[t]{2}{*}{ WAI } & $\begin{array}{l}\text { Environment } \\
\text { al Conditions }\end{array}$ & 517 & -.127 \\
\hline & & & $\begin{array}{l}\text { Physical } \\
\text { Demands }\end{array}$ & 517 & -.119 \\
\hline
\end{tabular}




\begin{tabular}{|c|c|c|c|c|c|}
\hline \multirow{19}{*}{$\begin{array}{l}\text { Work ability and its related } \\
\text { factors among workers in } \\
\text { small and medium } \\
\text { enterprises: Comparison } \\
\text { among four ASEAN } \\
\text { countries. Asia-Pacific } \\
\text { Journal of Public } \\
\text { Health, } 28(5), 438-449 . \\
\text { doi:http://dx.doi.org.proxy.1 } \\
\text { ib.pdx.edu/10.1177/101053 } \\
\text { 9516651957 }\end{array}$} & & & $\begin{array}{l}\text { Pain/Muscul } \\
\text { oskeletal } \\
\text { Symptoms }\end{array}$ & 517 & -.225 \\
\hline & & & Exercise & 517 & -.012 \\
\hline & & & Drinking & 517 & .036 \\
\hline & & & Smoking & 517 & .056 \\
\hline & & & Age & 517 & -.115 \\
\hline & \multirow[t]{7}{*}{$\begin{array}{l}\text { Mixed/Other } \\
\text { (Sample 2) }\end{array}$} & \multirow[t]{7}{*}{ WAI } & $\begin{array}{l}\text { Environment } \\
\text { al Conditions }\end{array}$ & 545 & -.104 \\
\hline & & & $\begin{array}{l}\text { Physical } \\
\text { Demands }\end{array}$ & 545 & -.060 \\
\hline & & & $\begin{array}{l}\text { Pain/Muscul } \\
\text { oskeletal } \\
\text { Symptoms }\end{array}$ & 545 & -.148 \\
\hline & & & Exercise & 545 & .173 \\
\hline & & & Drinking & 545 & -.083 \\
\hline & & & Smoking & 545 & -.098 \\
\hline & & & Age & 545 & -.080 \\
\hline & \multirow[t]{7}{*}{ Mixed/Other } & \multirow[t]{7}{*}{ WAI } & $\begin{array}{l}\text { Environment } \\
\text { al Conditions }\end{array}$ & 500 & -.188 \\
\hline & & & $\begin{array}{l}\text { Physical } \\
\text { Demands }\end{array}$ & 500 & -.064 \\
\hline & & & $\begin{array}{l}\text { Pain/Muscul } \\
\text { oskeletal } \\
\text { Symptoms }\end{array}$ & 500 & -.289 \\
\hline & & & Exercise & 500 & -.031 \\
\hline & & & Drinking & 500 & .065 \\
\hline & & & Smoking & 500 & .018 \\
\hline & & & Age & 500 & -.004 \\
\hline
\end{tabular}




\begin{tabular}{|c|c|c|c|c|c|}
\hline & Mixed/Other & WAI & $\begin{array}{l}\text { Environment } \\
\text { al Conditions }\end{array}$ & 536 & -.025 \\
\hline & & & $\begin{array}{l}\text { Physical } \\
\text { Demands }\end{array}$ & 536 & -.041 \\
\hline & & & $\begin{array}{l}\text { Pain/Muscul } \\
\text { oskeletal } \\
\text { Symptoms }\end{array}$ & 536 & -.258 \\
\hline & & & Exercise & 536 & .102 \\
\hline & & & Drinking & 536 & -.127 \\
\hline & & & Smoking & 536 & .026 \\
\hline & & & Age & 536 & -.022 \\
\hline Kaleta, D. O. R. O. T. A., & Mixed/Other & PWA & Exercise & 103 & .199 \\
\hline E. R. E. S. A., \& Jegier, A. & ( Sampie 1) & & BMI & 198 & -.300 \\
\hline $\begin{array}{l}\text { N. N. A. (2004). Leisure- } \\
\text { time physical activity, }\end{array}$ & & $\begin{array}{l}\text { both } \\
\text { subsets }\end{array}$ & $\begin{array}{l}\text { Physical } \\
\text { Ability }\end{array}$ & 198 & .400 \\
\hline and work ability: A study & & of & Exercise & 103 & .310 \\
\hline $\begin{array}{l}\text { in randomly selected } \\
\text { residents of Łódź. }\end{array}$ & & & Smoking & 103 & -.205 \\
\hline International Journal of & & & Age & 198 & -.600 \\
\hline $\begin{array}{l}\text { Occupational Medicine and } \\
\text { Environmental Health, }\end{array}$ & Mixed/Other & PWA & Exercise & 95 & .291 \\
\hline 17(4), 457-64. (Merged & & WAI & Exercise & 95 & .302 \\
\hline & & & Smoking & 95 & -.601 \\
\hline $\begin{array}{l}\text { Kaleta, D., Makowiec- } \\
\text { Dąbrowska, T., \& Jegier, } \\
\text { A. (2006). Lifestyle index } \\
\text { and work } \\
\text { ability. International } \\
\text { Journal of Occupational } \\
\text { Medicine and } \\
\text { Environmental } \\
\text { Health, 19(3), 170-177. }\end{array}$ & $\begin{array}{l}\text { See Kaleta et } \\
\text { al. (2004) }\end{array}$ & & & & \\
\hline & Blue Collar & WAI & Sleep & 399 & .157 \\
\hline
\end{tabular}




\begin{tabular}{|c|c|c|c|c|c|}
\hline \multirow{2}{*}{$\begin{array}{l}\text { Karttunen, J. P., \& } \\
\text { Rautiainen, R. H. (2009). } \\
\text { Work ability index among } \\
\text { Finnish dairy farmers. } \\
\text { Journal of Agricultural } \\
\text { Safety and Health, 15(4), } \\
\text { 353-364. }\end{array}$} & & & Drinking & 399 & .154 \\
\hline & & & Age & 399 & -.312 \\
\hline \multirow{6}{*}{$\begin{array}{l}\text { Karvala, K., Nordman, H., } \\
\text { Luukkonen, R., \& Uitti, J. } \\
\text { (2014). Asthma related to } \\
\text { workplace dampness and } \\
\text { impaired work ability. } \\
\text { International Archives of } \\
\text { Occupational and } \\
\text { Environmental Health, } \\
87(1), 1-11 .\end{array}$} & \multirow[t]{6}{*}{ Mixed/Other } & \multirow[t]{6}{*}{ PWA** } & $\begin{array}{l}\text { Supervisor } \\
\text { Support }\end{array}$ & 607 & .172 \\
\hline & & & $\begin{array}{l}\text { Organization } \\
\text { al Climate }\end{array}$ & 610 & .136 \\
\hline & & & $\begin{array}{l}\text { Symptoms - } \\
\text { Non Pain } \\
\text { related }\end{array}$ & 712 & -.462 \\
\hline & & & Depression & 875 & -.661 \\
\hline & & & Smoking & 690 & -.163 \\
\hline & & & Age & 728 & -.093 \\
\hline \multirow{4}{*}{$\begin{array}{l}\text { Kinnunen, U., Parkatti, T., } \\
\text { \& Rasku, A. (1994). } \\
\text { Occupational well-being } \\
\text { among aging teachers in } \\
\text { Finland. Scandinavian } \\
\text { Journal of Educational } \\
\text { Research, 38(3-4), 315- } \\
\text { 332. }\end{array}$} & \multirow[t]{4}{*}{ White Collar } & \multirow[t]{4}{*}{ WAI } & $\begin{array}{l}\text { Symptoms - } \\
\text { Non Pain } \\
\text { related }\end{array}$ & 1012 & -.560 \\
\hline & & & $\begin{array}{l}\text { Mental } \\
\text { Health }\end{array}$ & 1012 & .475 \\
\hline & & & Burnout & 1012 & -.500 \\
\hline & & & $\begin{array}{l}\text { Job Self- } \\
\text { Efficacy }\end{array}$ & 1012 & .310 \\
\hline $\begin{array}{l}\text { Koolhaas, W., van der } \\
\text { Klink, J. J., de Boer, M. R., } \\
\text { Groothoff, J. W., \& } \\
\text { Brouwer, S. (2014). } \\
\text { Chronic health conditions } \\
\text { and work ability in the } \\
\text { ageing workforce: the } \\
\text { impact of work conditions, } \\
\text { psychosocial factors and } \\
\text { perceived health. }\end{array}$ & Mixed/Other & $\mathrm{PWA}^{* *}$ & $\begin{array}{l}\text { Current } \\
\text { Health Status }\end{array}$ & 5247 & .303 \\
\hline
\end{tabular}




\begin{tabular}{|c|c|c|c|c|c|}
\hline $\begin{array}{l}\text { International Archives of } \\
\text { Occupational and } \\
\text { Environmental Health, } \\
87(4), 433-443 \text {. }\end{array}$ & & & & & \\
\hline \multirow{2}{*}{$\begin{array}{l}\text { Kujala, V., Tammelin, T., } \\
\text { Remes, J., Vammavaara, } \\
\text { E., Ek, E., \& Laitinen, J. } \\
\text { (2006). Work ability index } \\
\text { of young employees and } \\
\text { their sickness absence } \\
\text { during the following year. } \\
\text { Scandinavian Journal of } \\
\text { Work, Environment \& } \\
\text { Health, 31(1), 75-84. }\end{array}$} & Mixed/Other & WAI & $\begin{array}{l}\text { Future } \\
\text { Absenteeism }\end{array}$ & 2021 & $\begin{array}{l}- \\
0.16 \\
1\end{array}$ \\
\hline & Mixed/Other & WAI & $\begin{array}{l}\text { Future } \\
\text { Absenteeism }\end{array}$ & 1704 & $\begin{array}{l}- \\
0.17 \\
9\end{array}$ \\
\hline \multirow{2}{*}{$\begin{array}{l}\text { Kuoppala, J., Lamminpää, } \\
\text { A., Väänänen-Tomppo, I., } \\
\& \text { Hinkka, K. (2011). } \\
\text { Employee well-being and } \\
\text { sick leave, occupational } \\
\text { accident, and disability } \\
\text { pension: a cohort study of } \\
\text { civil servants. Journal of } \\
\text { Occupational and } \\
\text { Environmental } \\
\text { Medicine, 53(6), 633-640. }\end{array}$} & \multirow[t]{2}{*}{ Mixed/Other } & \multirow[t]{2}{*}{ WAI } & $\begin{array}{l}\text { Future } \\
\text { Absenteeism }\end{array}$ & 916 & -.238 \\
\hline & & & $\begin{array}{l}\text { Future } \\
\text { Disability }\end{array}$ & 916 & -.410 \\
\hline \multirow{2}{*}{$\begin{array}{l}\text { Kuusio, H., Heponiemi, T., } \\
\text { Aalto, A. M., Sinervo, T., } \\
\text { \& Elovainio, M. (2012). } \\
\text { Differences in Well-being } \\
\text { between GPs, Medical } \\
\text { Specialists, and Private } \\
\text { Physicians: The Role of } \\
\text { Psychosocial } \\
\text { Factors. Health Services } \\
\text { Research, 47(1), 68-85. }\end{array}$} & \multirow[t]{2}{*}{$\begin{array}{l}\text { Nurses/Healt } \\
\text { hcare }\end{array}$} & \multirow[t]{2}{*}{ PWA** } & $\begin{array}{l}\text { General } \\
\text { Health }\end{array}$ & 2047 & .540 \\
\hline & & & $\begin{array}{l}\text { Mental } \\
\text { Health }\end{array}$ & 2047 & .470 \\
\hline \multirow{2}{*}{$\begin{array}{l}\text { Labbafinejad, Y., Ghaffari, } \\
\text { M., Bahadori, B., } \\
\text { Mohammadi, S., Abdi, A., }\end{array}$} & \multirow[t]{2}{*}{ Blue Collar } & \multirow[t]{2}{*}{ WAI } & Sleep & 728 & .301 \\
\hline & & & BMI & 728 & .243 \\
\hline
\end{tabular}




\begin{tabular}{|c|c|c|c|c|c|}
\hline \multirow{3}{*}{$\begin{array}{l}\text { Namvar, M., \& Attarchi, } \\
\text { M. (2014). The effect of } \\
\text { sleep disorder on the work } \\
\text { ability of workers in a car } \\
\text { accessories manufacturing } \\
\text { plant. Medical Journal of } \\
\text { the Islamic Republic of } \\
\text { Iran, 28(1), 727-734. }\end{array}$} & & & Exercise & 728 & .021 \\
\hline & & & Smoking & 728 & -.165 \\
\hline & & & Age & 728 & -.093 \\
\hline \multirow{4}{*}{$\begin{array}{l}\text { Laitinen, J., Näyhä, S., \& } \\
\text { Kujala, V. (2005). Body } \\
\text { mass index and weight } \\
\text { change from adolescence } \\
\text { into adulthood, waist-to-hip } \\
\text { ratio and perceived work } \\
\text { ability among young } \\
\text { adults. International } \\
\text { Journal of Obesity, 29(6), } \\
697-702 \text {. }\end{array}$} & \multirow[t]{4}{*}{ Mixed/Other } & \multirow[t]{4}{*}{ WAI } & $\begin{array}{l}\text { Physical } \\
\text { Health }\end{array}$ & 2651 & .053 \\
\hline & & & BMI & 2651 & $\begin{array}{l}- \\
.041 \\
6\end{array}$ \\
\hline & & & Smoking & 5,436 & -.081 \\
\hline & & & Drinking & 5,436 & .056 \\
\hline \multirow{8}{*}{$\begin{array}{l}\text { Larsson, A., Karlqvist, L., } \\
\text { Westerberg, M., \& Gard, } \\
\text { G. (2012). Identifying work } \\
\text { ability promoting factors } \\
\text { for home care aides and } \\
\text { assistant nurses. BMC } \\
\text { Musculoskeletal Disorders, } \\
\text { 13(1), 1-11. }\end{array}$} & \multirow[t]{6}{*}{$\begin{array}{l}\text { Nurses/Healt } \\
\text { hcare }\end{array}$} & \multirow[t]{6}{*}{ PWA } & $\begin{array}{l}\text { Physical } \\
\text { Demands }\end{array}$ & 79 & -.300 \\
\hline & & & $\begin{array}{l}\text { Quantitative } \\
\text { Job Demands }\end{array}$ & 79 & -.200 \\
\hline & & & $\begin{array}{l}\text { Organization } \\
\text { al Climate }\end{array}$ & 79 & .240 \\
\hline & & & Age & 79 & .060 \\
\hline & & & $\begin{array}{l}\text { Pain/Muscul } \\
\text { oskeletal } \\
\text { Symptoms }\end{array}$ & 79 & -.460 \\
\hline & & & $\begin{array}{l}\text { Job Self- } \\
\text { Efficacy }\end{array}$ & 79 & .450 \\
\hline & \multirow[t]{2}{*}{$\begin{array}{l}\text { Nurses/Healt } \\
\text { hcare }\end{array}$} & \multirow[t]{2}{*}{ PWA } & $\begin{array}{l}\text { Physical } \\
\text { Demands }\end{array}$ & 58 & -.310 \\
\hline & & & $\begin{array}{l}\text { Quantitative } \\
\text { Job Demands }\end{array}$ & 58 & -.330 \\
\hline
\end{tabular}




\begin{tabular}{|c|c|c|c|c|c|}
\hline & & & $\begin{array}{l}\text { Organization } \\
\text { al Climate }\end{array}$ & 58 & .270 \\
\hline & & & $\begin{array}{l}\text { Job Self- } \\
\text { Efficacy }\end{array}$ & 58 & .380 \\
\hline & & & Age & 58 & -.320 \\
\hline & & & $\begin{array}{l}\text { Pain/Muscul } \\
\text { oskeletal } \\
\text { Symptoms }\end{array}$ & 58 & -.280 \\
\hline $\begin{array}{l}\text { Łastowiecka, E., Bugajska, } \\
\text { J., Najmiec, A., Rell- } \\
\text { Bakalarska, M., Bownik, I., }\end{array}$ & Mixed/Other & WAI & $\begin{array}{l}\text { Current } \\
\text { Health Status }\end{array}$ & 166 & .134 \\
\hline $\begin{array}{l}\text { \& Jędryka-Góral, A. } \\
\text { (2006). Occupational work } \\
\text { and quality of life in } \\
\text { osteoarthritis } \\
\text { patients. Rheumatology } \\
\text { International, 27(2), 131- } \\
139 .\end{array}$ & & & Age & 164 & .119 \\
\hline $\begin{array}{l}\text { Leggett, S., Van Der Zee- } \\
\text { Neuen, A., Boonen, A., } \\
\text { Beaton, D. E., Bojinca, M., } \\
\text { Bosworth, A., \& Lacaille, }\end{array}$ & $\begin{array}{l}\text { Nurses/Healt } \\
\text { hcare }\end{array}$ & PWA* & $\begin{array}{l}\text { General } \\
\text { Health }\end{array}$ & 65 & .610 \\
\hline $\begin{array}{l}\text { D. (2016). Test-retest } \\
\text { reliability and correlations } \\
\text { of } 5 \text { global measures } \\
\text { addressing at-work } \\
\text { productivity loss in patients } \\
\text { with rheumatic } \\
\text { diseases. The Journal of } \\
\text { Rheumatology, 43(2), 433- } \\
439 .\end{array}$ & & & $\begin{array}{l}\text { Job } \\
\text { performance }\end{array}$ & 62 & .599 \\
\hline $\begin{array}{l}\text { Leijten, F. R. M., van, d. } \\
\text { H., Ybema, J. F., van, d. B., } \\
\text { Robroek, S. J. W., \& } \\
\text { Burdorf, A. (2014). The } \\
\text { influence of chronic health } \\
\text { problems on work ability } \\
\text { and productivity at work: A }\end{array}$ & Mixed/Other & PWA & $\begin{array}{l}\text { Job } \\
\text { performance }\end{array}$ & 8411 & .230 \\
\hline
\end{tabular}




\begin{tabular}{|c|c|c|c|c|c|}
\hline $\begin{array}{l}\text { longitudinal study among } \\
\text { older } \\
\text { employees. Scandinavian } \\
\text { Journal of Work, } \\
\text { Environment \& } \\
\text { Health, } 40(5), 473-482 . \\
\text { doi:http://dx.doi.org.proxy.1 } \\
\text { ib.pdx.edu/10.5271/sjweh.3 } \\
444\end{array}$ & & & & & \\
\hline \multirow{6}{*}{$\begin{array}{l}\text { Li, H., Liu, Z., Liu, R., Li, } \\
\text { L., \& Lin, A. (2015). The } \\
\text { relationship between work } \\
\text { stress and work ability } \\
\text { among power supply } \\
\text { workers in Guangdong, } \\
\text { China: a cross-sectional } \\
\text { study. BMC Public } \\
\text { Health, 16(1), 123-131. }\end{array}$} & \multirow[t]{6}{*}{ Mixed/Other } & \multirow[t]{6}{*}{ PWA } & Job Demands & 805 & -.145 \\
\hline & & & Job Control & 805 & .250 \\
\hline & & & Reward & 805 & .220 \\
\hline & & & $\begin{array}{l}\text { Job } \\
\text { Resources } \\
\text { (Other) }\end{array}$ & 805 & .170 \\
\hline & & & $\begin{array}{l}\text { Mental } \\
\text { Health }\end{array}$ & 805 & .430 \\
\hline & & & Age & 805 & -.020 \\
\hline $\begin{array}{l}\text { Lian, Y., Xiao, J., Liu, Y., } \\
\text { Ning, L., Guan, S., Ge, H., } \\
\text { \& Liu, J. (2015). } \\
\text { Associations between } \\
\text { insomnia, sleep duration } \\
\text { and poor work } \\
\text { ability. Journal of } \\
\text { Psychosomatic } \\
\text { Research, 78(1), 45-51. }\end{array}$ & Blue Collar & WAI & Sleep & 2798 & .170 \\
\hline \multirow{2}{*}{$\begin{array}{l}\text { Lin, S., Wang, Z., \& Wang, } \\
\text { M. (2006). Work ability of } \\
\text { workers in western China: } \\
\text { reference data. } \\
\text { Occupational Medicine, } \\
56(2), 89-93 .\end{array}$} & Mixed/Other & WAI & Age & 4405 & -.146 \\
\hline & Mixed/Other & WAI & Age & 5813 & -.202 \\
\hline $\begin{array}{l}\text { Lindbohm, M. L., Taskila, } \\
\text { T., Kuosma, E., Hietanen, } \\
\text { P., Carlsen, K., }\end{array}$ & Mixed/Other & PWA** & $\begin{array}{l}\text { Current } \\
\text { Health Status }\end{array}$ & 4158 & .077 \\
\hline
\end{tabular}




\begin{tabular}{|c|c|c|c|c|c|}
\hline $\begin{array}{l}\text { Gudbergsson, S., \& } \\
\text { Gunnarsdottir, H. (2012). } \\
\text { Work ability of survivors of } \\
\text { breast, prostate, and } \\
\text { testicular cancer in Nordic } \\
\text { countries: a NOCWO } \\
\text { study. Journal of Cancer } \\
\text { Survivorship, 6(1), 72-81. }\end{array}$ & & & & & \\
\hline \multirow{2}{*}{$\begin{array}{l}\text { Lindegård, A., Larsman, P., } \\
\text { Hadzibajramovic, E., \& } \\
\text { Ahlborg, G. (2014). The } \\
\text { influence of perceived } \\
\text { stress and musculoskeletal } \\
\text { pain on work performance } \\
\text { and work ability in Swedish } \\
\text { health care } \\
\text { workers. International } \\
\text { Archives of Occupational } \\
\text { and Environmental } \\
\text { Health, 87(4), 373-379. }\end{array}$} & \multirow[t]{2}{*}{$\begin{array}{l}\text { Nurses/Healt } \\
\text { hcare }\end{array}$} & \multirow[t]{2}{*}{ PWA* } & $\begin{array}{l}\text { Pain/Muscul } \\
\text { oskeletal } \\
\text { Symptoms }\end{array}$ & 740 & -.166 \\
\hline & & & $\begin{array}{l}\text { Perceived } \\
\text { Stress }\end{array}$ & 741 & -.094 \\
\hline $\begin{array}{l}\text { Lindström, I., Pallasaho, P., } \\
\text { Luukkonen, R., Suojalehto, } \\
\text { H., Karjalainen, J., } \\
\text { Lauerma, A., \& } \\
\text { Karjalainen, A. (2011). } \\
\text { Reduced work ability in } \\
\text { middle-aged men with } \\
\text { asthma from youth-a 20- } \\
\text { year follow-up. Respiratory } \\
\text { Medicine, 105(6), 950-955. }\end{array}$ & Mixed/Other & PWA** & $\begin{array}{l}\text { Current } \\
\text { Health Status }\end{array}$ & 1001 & .127 \\
\hline $\begin{array}{l}\text { Löve, J., Holmgren, K., } \\
\text { Torén, K., \& Hensing, G. } \\
\text { (2012). Can work ability } \\
\text { explain the social gradient } \\
\text { in sickness absence: a study } \\
\text { of a general population in } \\
\text { Sweden. BMC Public } \\
\text { Health, } 12(1), 1-9 \text {. }\end{array}$ & Mixed/Other & PWA & $\begin{array}{l}\text { Current } \\
\text { Health Status }\end{array}$ & 5807 & .243 \\
\hline
\end{tabular}




\begin{tabular}{|c|c|c|c|c|c|}
\hline \multirow{10}{*}{$\begin{array}{l}\text { Lundin, A., Kjellberg, K., } \\
\text { Leijon, O., Punnett, L., \& } \\
\text { Hemmingsson, T. (2016). } \\
\text { The association between } \\
\text { self-assessed future work } \\
\text { ability and long-term } \\
\text { sickness absence, disability } \\
\text { pension and unemployment } \\
\text { in a general working } \\
\text { population: A 7-year } \\
\text { follow-up study. Journal of } \\
\text { Occupational } \\
\text { Rehabilitation, 26(2), 195- } \\
\text { 203. } \\
\text { doi:http://dx.doi.org.proxy.1 } \\
\text { ib.pdx.edu/10.1007/s10926 } \\
\text {-015-9603-4 }\end{array}$} & \multirow[t]{10}{*}{ Mixed/Other } & \multirow[t]{10}{*}{ PWA } & $\begin{array}{l}\text { Physical } \\
\text { Demands }\end{array}$ & 12013 & -.158 \\
\hline & & & $\begin{array}{l}\text { Quantitative } \\
\text { Job Demands }\end{array}$ & 12064 & -.143 \\
\hline & & & Job control & $\begin{array}{l}12,07 \\
5\end{array}$ & .178 \\
\hline & & & $\begin{array}{l}\text { Current } \\
\text { Health Status }\end{array}$ & 12064 & .158 \\
\hline & & & $\begin{array}{l}\text { Previous } \\
\text { Absenteeism }\end{array}$ & 12064 & -.106 \\
\hline & & & $\begin{array}{l}\text { Mental } \\
\text { Health }\end{array}$ & 12065 & .189 \\
\hline & & & $\begin{array}{l}\text { Pain/Muscul } \\
\text { oskeletal } \\
\text { Symptoms }\end{array}$ & 12064 & -.200 \\
\hline & & & Drinking & 12064 & -.063 \\
\hline & & & Smoking & 12064 & -.103 \\
\hline & & & Age & 12065 & .013 \\
\hline \multirow{2}{*}{$\begin{array}{l}\text { Lundin, A., Leijon, O., } \\
\text { Vaez, M., Hallgren, M., \& } \\
\text { Torgén, M. (2017). } \\
\text { Predictive validity of the } \\
\text { Work Ability Index and its } \\
\text { individual items in the } \\
\text { general } \\
\text { population. Scandinavian } \\
\text { Journal of Public } \\
\text { Health, 45(4), 350-356. }\end{array}$} & \multirow[t]{2}{*}{ Mixed/Other } & WAI & $\begin{array}{l}\text { Future } \\
\text { Absenteeism }\end{array}$ & 1786 & -.298 \\
\hline & & PWA* & $\begin{array}{l}\text { Future } \\
\text { Absenteeism }\end{array}$ & 1786 & -.206 \\
\hline $\begin{array}{l}\text { Mache, S., Danzer, G., } \\
\text { Klapp, B. F., \& Groneberg, }\end{array}$ & $\begin{array}{l}\text { Nurses/Healt } \\
\text { hcare }\end{array}$ & WAI & $\begin{array}{l}\text { Quantitative } \\
\text { Job Demands }\end{array}$ & 123 & -.180 \\
\hline
\end{tabular}




\begin{tabular}{|c|c|c|c|c|c|}
\hline \multirow{11}{*}{$\begin{array}{l}\text { D. A. (2013). Surgeons' } \\
\text { work ability and } \\
\text { performance in surgical } \\
\text { care: relations between } \\
\text { organisational predictors, } \\
\text { work engagement and work } \\
\text { ability. Langenbeck's } \\
\text { Archives of Surgery, } \\
\text { 398(2), 317-325. }\end{array}$} & & & $\begin{array}{l}\text { Mental/Emot } \\
\text { ional } \\
\text { Demands }\end{array}$ & 123 & -.237 \\
\hline & & & Job Control & 123 & .355 \\
\hline & & & $\begin{array}{l}\text { Organization } \\
\text { al Climate }\end{array}$ & 123 & .200 \\
\hline & & & $\begin{array}{l}\text { Task } \\
\text { Resources }\end{array}$ & 123 & .270 \\
\hline & & & $\begin{array}{l}\text { Job } \\
\text { Resources } \\
\text { (Other) }\end{array}$ & 123 & .360 \\
\hline & & & $\begin{array}{l}\text { Core Self } \\
\text { Evaluations }\end{array}$ & 123 & .140 \\
\hline & & & $\begin{array}{l}\text { Positive } \\
\text { Affect }\end{array}$ & 123 & .150 \\
\hline & & & Resilience & 123 & .380 \\
\hline & & & $\begin{array}{l}\text { Negative } \\
\text { Traits/Emoti } \\
\text { ons }\end{array}$ & 123 & -.100 \\
\hline & & & Age & 123 & -.227 \\
\hline & & & $\begin{array}{l}\text { Work } \\
\text { Motivation }\end{array}$ & 123 & .430 \\
\hline \multirow{2}{*}{$\begin{array}{l}\text { Madeleine, P., Vangsgaard, } \\
\text { S., Andersen, J. H., Ge, H. } \\
\text { Y., \& Arendt-Nielsen, L. } \\
\text { (2013). Computer work and } \\
\text { self-reported variables on } \\
\text { anthropometrics, computer } \\
\text { usage, work ability, } \\
\text { productivity, pain, and } \\
\text { physical activity. BMC } \\
\text { Musculoskeletal Disorders, } \\
\text { 14(1), 1-9. }\end{array}$} & White Collar & PWA & $\begin{array}{l}\text { Pain/Muscul } \\
\text { oskeletal } \\
\text { Symptoms }\end{array}$ & 140 & -.400 \\
\hline & White Collar & PWA & $\begin{array}{l}\text { Pain/Muscul } \\
\text { oskeletal } \\
\text { Symptoms }\end{array}$ & 549 & -.470 \\
\hline
\end{tabular}




\begin{tabular}{|c|c|c|c|c|c|}
\hline $\begin{array}{l}\text { Magnago, T. S. B. D. S., } \\
\text { Lima, A. C. S. D., } \\
\text { Prochnow, A., Ceron, M. } \\
\text { D. D. S., Tavares, J. P., \& } \\
\text { Urbanetto, J. D. S. (2012). } \\
\text { Intensity of } \\
\text { musculoskeletal pain and } \\
\text { (in) ability to work in } \\
\text { nursing. Revista Latino- } \\
\text { Americana de Enfermagem, } \\
\text { 20(6), 1125-1133. }\end{array}$ & $\begin{array}{l}\text { Nurses/Healt } \\
\text { hcare }\end{array}$ & WAI & $\begin{array}{l}\text { Pain/Muscul } \\
\text { oskeletal } \\
\text { Symptoms }\end{array}$ & 262 & -.401 \\
\hline $\begin{array}{l}\text { Marklund, S., Bolin, M., \& } \\
\text { von Essen, J. (2008). Can } \\
\text { individual health } \\
\text { differences be explained by } \\
\text { workplace } \\
\text { characteristics?-A } \\
\text { multilevel analysis. Social } \\
\text { Science \& Medicine, 66(3), } \\
650-662 .\end{array}$ & Mixed/Other & WAI & $\begin{array}{l}\text { Task } \\
\text { Resources }\end{array}$ & 4306 & .062 \\
\hline \multirow{2}{*}{$\begin{array}{l}\text { Marqueze, E. C., Voltz, G. } \\
\text { P., Borges, F. N., \& } \\
\text { Moreno, C. R. (2008). A 2- } \\
\text { year follow-up study of } \\
\text { work ability among college } \\
\text { educators. Applied } \\
\text { Ergonomics, } 39(5), 640- \\
645 .\end{array}$} & \multirow[t]{2}{*}{ White Collar } & \multirow[t]{2}{*}{ WAI } & Age & 60 & .300 \\
\hline & & & $\begin{array}{l}\text { Job } \\
\text { Satisfaction }\end{array}$ & 154 & .230 \\
\hline \multirow{3}{*}{$\begin{array}{l}\text { Martinez, M. C., Latorre, } \\
\text { M. D. R. D. D., \& Fischer, } \\
\text { F. M. (2009). Validity and } \\
\text { reliability of the Brazilian } \\
\text { version of the Work Ability } \\
\text { Index questionnaire. }\end{array}$} & \multirow[t]{3}{*}{ Mixed/Other } & \multirow[t]{3}{*}{ WAI } & $\begin{array}{l}\text { General } \\
\text { Health }\end{array}$ & 475 & .490 \\
\hline & & & $\begin{array}{l}\text { Mental } \\
\text { Health }\end{array}$ & 475 & .400 \\
\hline & & & $\begin{array}{l}\text { Physical } \\
\text { health }\end{array}$ & 475 & .480 \\
\hline
\end{tabular}




\begin{tabular}{|c|c|c|c|c|c|}
\hline \multirow[t]{2}{*}{$\begin{array}{l}\text { Revista de Saude Publica, } \\
43(3), 525-532 .\end{array}$} & & & $\begin{array}{l}\text { Pain/Muscul } \\
\text { oskeletal } \\
\text { Symptoms }\end{array}$ & 475 & -.382 \\
\hline & & & Fatigue & 475 & -.390 \\
\hline \multirow[t]{4}{*}{$\begin{array}{l}\text { McGonagle et al. } \\
\text { (Unpublished) }\end{array}$} & \multirow[t]{4}{*}{ Mixed/Other } & \multirow[t]{4}{*}{ PWA } & $\begin{array}{l}\text { Negative } \\
\text { Traits/Emoti } \\
\text { ons }\end{array}$ & 355 & .430 \\
\hline & & & $\begin{array}{l}\text { Pain/Muscul } \\
\text { oskeletal } \\
\text { Symptoms }\end{array}$ & 355 & -.430 \\
\hline & & & Burnout & 355 & -.490 \\
\hline & & & $\begin{array}{l}\text { Job } \\
\text { Satisfaction }\end{array}$ & 355 & .320 \\
\hline \multirow{5}{*}{$\begin{array}{l}\text { McGonagle, A. K., \& } \\
\text { Barnes-Farrell, J. L. (2014). } \\
\text { Chronic illness in the } \\
\text { workplace: Stigma, identity } \\
\text { threat and strain. Stress and } \\
\text { Health, 30(4), 310-321. }\end{array}$} & \multirow[t]{5}{*}{ Mixed/Other } & \multirow[t]{5}{*}{ PWA** } & Job Control & 203 & . 240 \\
\hline & & & $\begin{array}{l}\text { Supervisor } \\
\text { Support }\end{array}$ & 203 & . 180 \\
\hline & & & $\begin{array}{l}\text { Perceived } \\
\text { Stress }\end{array}$ & 203 & -.370 \\
\hline & & & $\begin{array}{l}\text { Job Self- } \\
\text { Efficacy }\end{array}$ & 203 & .260 \\
\hline & & & $\begin{array}{l}\text { General } \\
\text { Health }\end{array}$ & 203 & .360 \\
\hline \multirow{4}{*}{$\begin{array}{l}\text { McGonagle, A. K., Barnes- } \\
\text { Farrell, J. L., Di Milia, L., } \\
\text { Fischer, F. M., Hobbs, B. } \\
\text { B., Iskra-Golec, I., \& } \\
\text { Smith, L. (2014). Demands, } \\
\text { resources, and work ability: } \\
\text { A cross-national } \\
\text { examination of health care } \\
\text { workers. European Journal } \\
\text { of Work and } \\
\text { Organizational }\end{array}$} & \multirow[t]{4}{*}{$\begin{array}{l}\text { Nurses/Healt } \\
\text { hcare }\end{array}$} & \multirow[t]{4}{*}{ PWA } & $\begin{array}{l}\text { Environment } \\
\text { al Conditions }\end{array}$ & 1225 & -.200 \\
\hline & & & $\begin{array}{l}\text { Mental/Emot } \\
\text { ional } \\
\text { Demands }\end{array}$ & 1225 & .000 \\
\hline & & & $\begin{array}{l}\text { Physical } \\
\text { Demands }\end{array}$ & 1225 & -.090 \\
\hline & & & $\begin{array}{l}\text { Quantitative } \\
\text { Job Demands }\end{array}$ & 1225 & -.140 \\
\hline
\end{tabular}




\begin{tabular}{|c|c|c|c|c|c|}
\hline \multirow[t]{4}{*}{$\begin{array}{l}\text { Psychology, 23(6), 830- } \\
846 .\end{array}$} & & & $\begin{array}{l}\text { Supervisor } \\
\text { Support }\end{array}$ & 1225 & .150 \\
\hline & & & $\begin{array}{l}\text { Task } \\
\text { Resources }\end{array}$ & 1225 & .250 \\
\hline & & & $\begin{array}{l}\text { General } \\
\text { Health }\end{array}$ & 1225 & .420 \\
\hline & & & Age & 1225 & .050 \\
\hline \multirow{12}{*}{$\begin{array}{l}\text { McGonagle, A. K., Fisher, } \\
\text { G. G., Barnes-Farrell, J. L., } \\
\& \text { Grosch, J. W. (2014). } \\
\text { Individual and work factors } \\
\text { related to perceived work } \\
\text { ability and labor force } \\
\text { outcomes. Journal of } \\
\text { Applied Psychology, } \\
\text { 100(2), 376-398. }\end{array}$} & \multirow[t]{12}{*}{$\begin{array}{l}\text { Mixed/Other } \\
\text { (Sample 1) }\end{array}$} & \multirow[t]{12}{*}{ PWA } & $\begin{array}{l}\text { Workplace } \\
\text { Mistreatment }\end{array}$ & 1656 & .040 \\
\hline & & & $\begin{array}{l}\text { Environment } \\
\text { al Conditions }\end{array}$ & 1656 & -.100 \\
\hline & & & $\begin{array}{l}\text { Physical } \\
\text { Demands }\end{array}$ & 1656 & -.135 \\
\hline & & & $\begin{array}{l}\text { Mental/Emot } \\
\text { ional } \\
\text { Demands }\end{array}$ & 1656 & -.110 \\
\hline & & & $\begin{array}{l}\text { Quantitative } \\
\text { Job Demands }\end{array}$ & 1656 & -.087 \\
\hline & & & Job Control & 1656 & .177 \\
\hline & & & $\begin{array}{l}\text { Supervisor } \\
\text { Support }\end{array}$ & 1656 & .210 \\
\hline & & & $\begin{array}{l}\text { Coworker } \\
\text { Support }\end{array}$ & 1656 & .230 \\
\hline & & & $\begin{array}{l}\text { Conscientiou } \\
\text { sness }\end{array}$ & 1656 & .340 \\
\hline & & & $\begin{array}{l}\text { Positive } \\
\text { Affect }\end{array}$ & 1656 & .380 \\
\hline & & & $\begin{array}{l}\text { Negative } \\
\text { Traits/Emoti } \\
\text { ons }\end{array}$ & 1656 & -.290 \\
\hline & & & $\begin{array}{l}\text { General } \\
\text { Health }\end{array}$ & 1656 & .360 \\
\hline
\end{tabular}




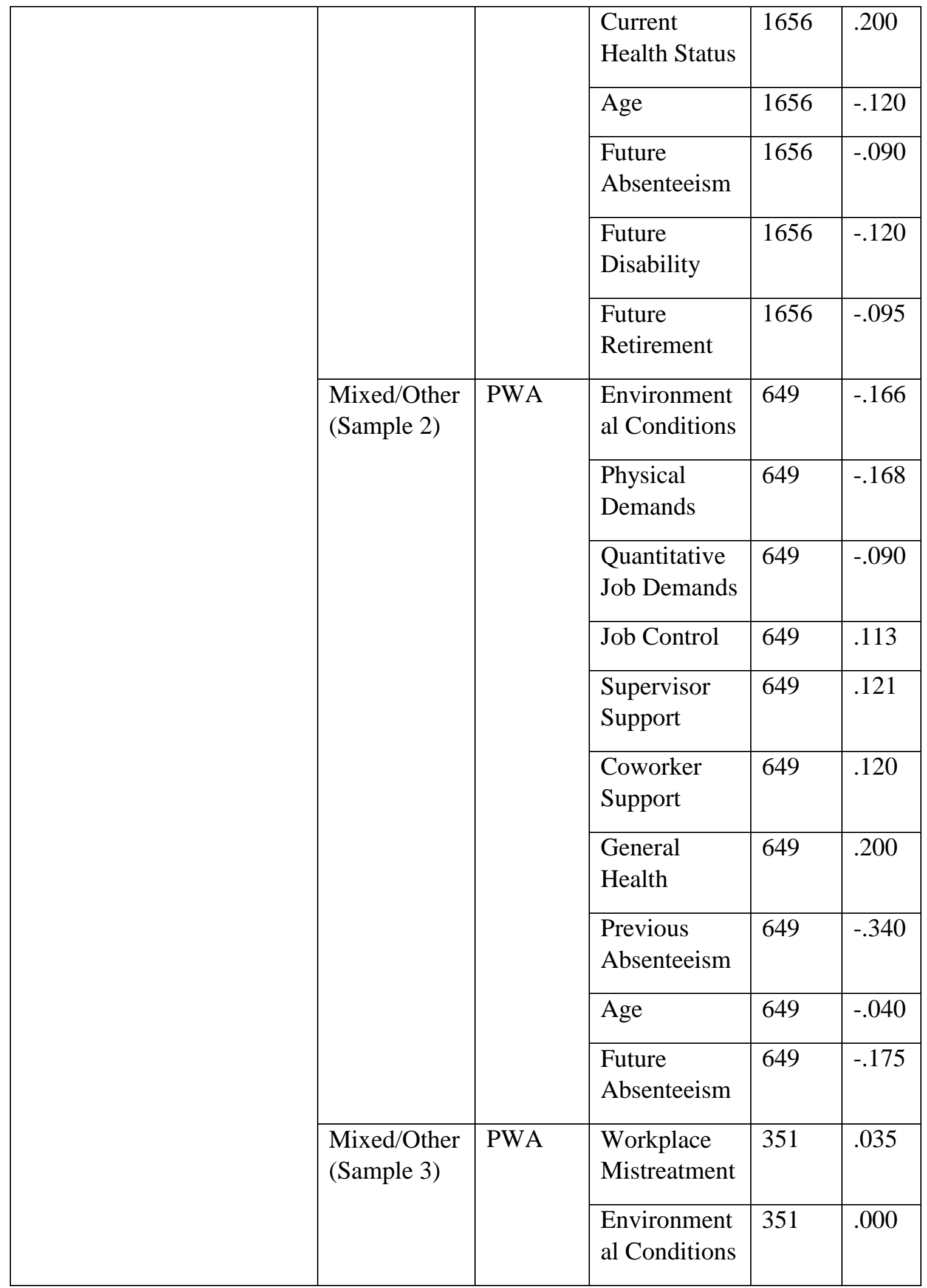




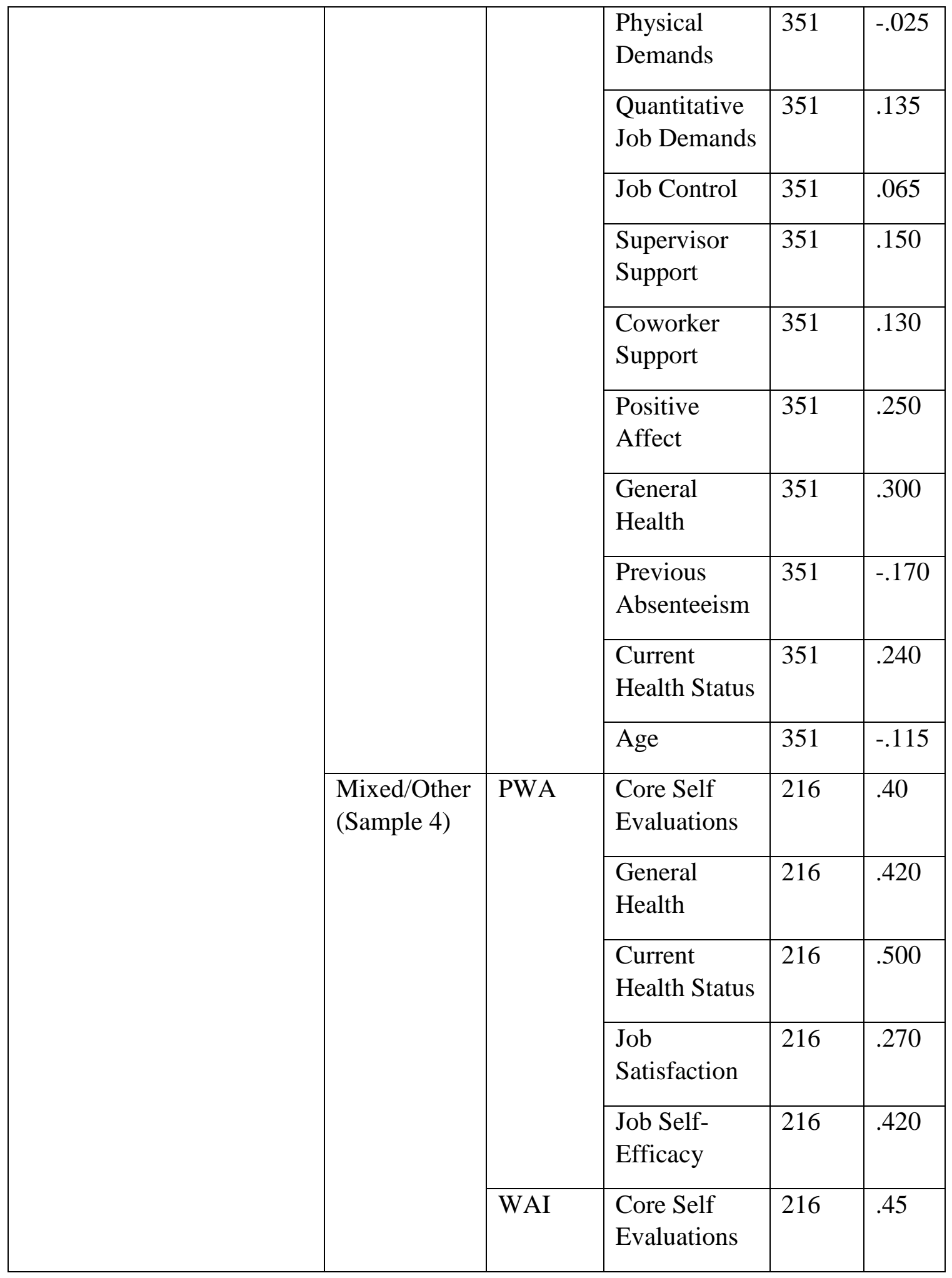




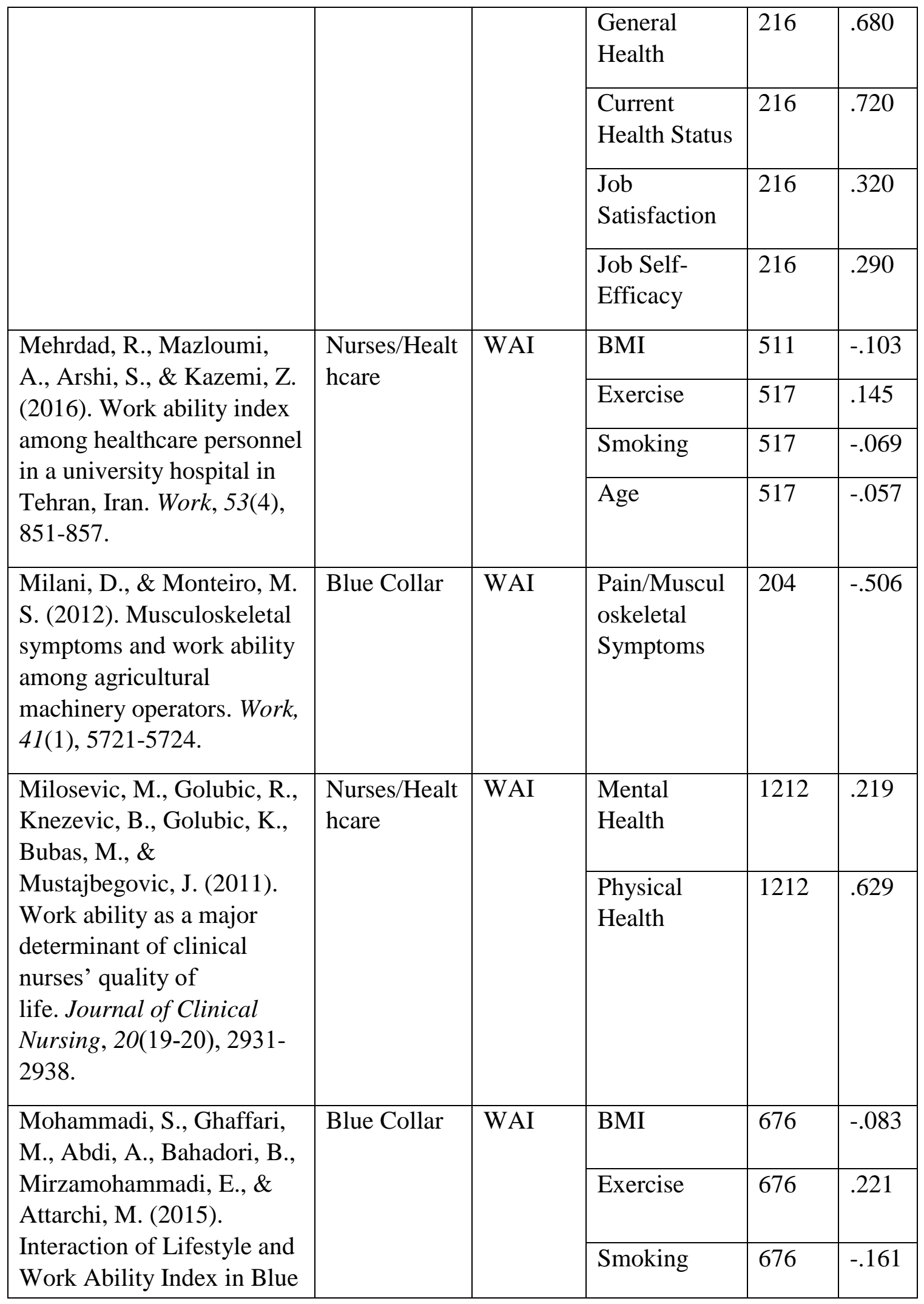




\begin{tabular}{|c|c|c|c|c|c|}
\hline \multicolumn{6}{|l|}{$\begin{array}{l}\text { Collar Workers. Global } \\
\text { Journal of Health Science, } \\
\text { 7(3), 90-97. }\end{array}$} \\
\hline \multirow{5}{*}{$\begin{array}{l}\text { Möller, L. M., Brands, R., } \\
\text { Sluiter, J. K., Schouten, J., } \\
\text { Wit, F. W., Reiss, P., \& } \\
\text { Stolte, I. G. (2016). } \\
\text { Prevalence and } \\
\text { determinants of insufficient } \\
\text { work ability in older HIV- } \\
\text { positive and HIV-negative } \\
\text { workers. International } \\
\text { Archives of Occupational } \\
\text { and Environmental } \\
\text { Health, 89(4), 699-709. }\end{array}$} & \multirow[t]{5}{*}{ Mixed/Other } & \multirow[t]{5}{*}{ PWA** } & $\begin{array}{l}\text { Current } \\
\text { Health Status }\end{array}$ & 623 & .086 \\
\hline & & & $\begin{array}{l}\text { Previous } \\
\text { Abstenteeism }\end{array}$ & 623 & -.160 \\
\hline & & & Depression & 623 & -.345 \\
\hline & & & Exercise & 623 & .072 \\
\hline & & & Drinking & 623 & -.066 \\
\hline $\begin{array}{l}\text { Momsen, A. M. H., Jensen, } \\
\text { O. K., Nielsen, C. V., \& } \\
\text { Jensen, C. (2014). Multiple } \\
\text { somatic symptoms in } \\
\text { employees participating in } \\
\text { a randomized controlled } \\
\text { trial associated with } \\
\text { sickness absence because } \\
\text { of nonspecific low back } \\
\text { pain. The Spine } \\
\text { Journal, 14(12), 2868- } \\
\text { 2876. }\end{array}$ & Mixed/Other & PWA & $\begin{array}{l}\text { Symptoms - } \\
\text { Non Pain } \\
\text { related }\end{array}$ & 282 & -.205 \\
\hline \multirow{4}{*}{$\begin{array}{l}\text { Monteiro, M. S., Ilmarinen, } \\
\text { J., \& Filho, H. R. C. } \\
\text { (2006). Work ability of } \\
\text { workers in different age } \\
\text { groups in a public health } \\
\text { institution in } \\
\text { Brazil. International } \\
\text { Journal of Occupational } \\
\text { Safety and } \\
\text { Ergonomics, 12(4), 417- } \\
427 . \dagger\end{array}$} & \multirow[t]{4}{*}{ Mixed/Other } & \multirow[t]{4}{*}{ WAI } & BMI & 628 & -.085 \\
\hline & & & Exercise & 651 & .113 \\
\hline & & & Smoking & 651 & -.041 \\
\hline & & & Age & 866 & -.050 \\
\hline
\end{tabular}




\begin{tabular}{|c|c|c|c|c|c|}
\hline \multirow{2}{*}{$\begin{array}{l}\text { Monteiro, M. S., \& } \\
\text { Alexandre, N. M. C. } \\
\text { (2009). Work ability and } \\
\text { low back pain among } \\
\text { workers from a public } \\
\text { health institution. Revista } \\
\text { Gaúcha de Enfermagem, } \\
\text { 30(2), 297-311.† }\end{array}$} & \multirow[t]{2}{*}{ Mixed/Other } & WAI & $\begin{array}{l}\text { Pain/Muscul } \\
\text { oskeletal } \\
\text { Symptoms }\end{array}$ & 651 & -.468 \\
\hline & & PWA* & $\begin{array}{l}\text { Pain/Muscul } \\
\text { oskeletal } \\
\text { Symptoms }\end{array}$ & 651 & -.163 \\
\hline \multirow[t]{13}{*}{ Cadiz et al. (Unpublished) } & \multirow[t]{13}{*}{ Mixed/Other } & \multirow[t]{13}{*}{ PWA } & Job Control & 503 & .328 \\
\hline & & & $\begin{array}{l}\text { Supervisor } \\
\text { Support }\end{array}$ & 503 & .364 \\
\hline & & & $\begin{array}{l}\text { Justice } \\
\text { Perceptions }\end{array}$ & 503 & .255 \\
\hline & & & $\begin{array}{l}\text { Task } \\
\text { Resources }\end{array}$ & 503 & .232 \\
\hline & & & $\begin{array}{l}\text { Core Self- } \\
\text { Evaluations }\end{array}$ & 503 & .474 \\
\hline & & & $\begin{array}{l}\text { SOC } \\
\text { Behaviors }\end{array}$ & 503 & .284 \\
\hline & & & Burnout & 303 & -.396 \\
\hline & & & $\begin{array}{l}\text { Perceived } \\
\text { Stress }\end{array}$ & 303 & -.428 \\
\hline & & & Motivation & 503 & .393 \\
\hline & & & $\begin{array}{l}\text { Job } \\
\text { Satisfaction }\end{array}$ & 303 & .233 \\
\hline & & & $\begin{array}{l}\text { Organization } \\
\text { al } \\
\text { Commitment }\end{array}$ & 303 & .220 \\
\hline & & & $\begin{array}{l}\text { Job } \\
\text { Performance }\end{array}$ & 303 & .482 \\
\hline & & & $\begin{array}{l}\text { Retirement } \\
\text { Intentions }\end{array}$ & 303 & -.206 \\
\hline
\end{tabular}




\begin{tabular}{|c|c|c|c|c|c|}
\hline & & & $\begin{array}{l}\text { Turnover } \\
\text { Intentions }\end{array}$ & 303 & -.270 \\
\hline \multirow{2}{*}{$\begin{array}{l}\text { Müller, A., Heiden, B., } \\
\text { Herbig, B., Poppe, F., \& } \\
\text { Angerer, P. (2016). } \\
\text { Improving well-being at } \\
\text { work: A randomized } \\
\text { controlled intervention } \\
\text { based on selection, } \\
\text { optimization, and } \\
\text { compensation. Journal of } \\
\text { Occupational Health } \\
\text { Psychology, 21(2), 169- } \\
\text { 182. }\end{array}$} & \multirow[t]{2}{*}{$\begin{array}{l}\text { Nurses/Healt } \\
\text { hcare }\end{array}$} & \multirow[t]{2}{*}{ PWA } & Job Control & 57 & .199 \\
\hline & & & $\begin{array}{l}\text { Mental } \\
\text { Health }\end{array}$ & 57 & .467 \\
\hline \multirow{3}{*}{$\begin{array}{l}\text { Müller, A., Weigl, M., } \\
\text { Heiden, B., Glaser, J., \& } \\
\text { Angerer, P. (2012). } \\
\text { Promoting work ability and } \\
\text { well-being in hospital } \\
\text { nursing: The interplay of } \\
\text { age, job control, and } \\
\text { successful ageing } \\
\text { strategies. Work, 4l(1), } \\
\text { 5137-5144. }\end{array}$} & \multirow[t]{3}{*}{$\begin{array}{l}\text { Nurses/Healt } \\
\text { hcare }\end{array}$} & \multirow[t]{3}{*}{ PWA } & Job Control & 438 & .180 \\
\hline & & & Exercise & 438 & .170 \\
\hline & & & Age & 438 & -.120 \\
\hline \multirow{3}{*}{$\begin{array}{l}\text { Müller, A., Weigl, M., } \\
\text { Heiden, B., Herbig, B., } \\
\text { Glaser, J., \& Angerer, P. } \\
\text { (2013). Selection, } \\
\text { optimization, and } \\
\text { compensation in nursing: } \\
\text { exploration of job-specific } \\
\text { strategies, scale } \\
\text { development, and age- } \\
\text { specific associations to } \\
\text { work ability. Journal of } \\
\text { Advanced Nursing, 69(7), } \\
\text { 1630-1642. }\end{array}$} & \multirow[t]{3}{*}{$\begin{array}{l}\text { Nurses/Healt } \\
\text { hcare }\end{array}$} & \multirow[t]{3}{*}{ PWA* } & $\begin{array}{l}\text { Quantitative } \\
\text { Job Demands }\end{array}$ & 420 & -.160 \\
\hline & & & $\begin{array}{l}\text { Physical } \\
\text { Demands }\end{array}$ & 420 & -.170 \\
\hline & & & $\begin{array}{l}\text { SOC } \\
\text { Behaviors }\end{array}$ & 420 & .174 \\
\hline
\end{tabular}




\begin{tabular}{|c|c|c|c|c|c|}
\hline $\begin{array}{l}\text { Neri, L., Gallieni, M., } \\
\text { Cozzolino, M., Rocca-rey, } \\
\text { L., Brambilla, G., Colombi, } \\
\text { A., \& Brancaccio, D. } \\
\text { (2005). work ability and } \\
\text { health status in dialysis } \\
\text { patients. The International } \\
\text { Journal of Artificial } \\
\text { Organs, 28(6), 554-556. }\end{array}$ & Mixed/Other & WAI & $\begin{array}{l}\text { General } \\
\text { Health }\end{array}$ & 40 & .530 \\
\hline \multirow{9}{*}{$\begin{array}{l}\text { Netterstrøm, B., Eller, N. } \\
\text { H., \& Borritz, M. (2015). } \\
\text { Prognostic factors of } \\
\text { returning to work after sick } \\
\text { leave due to work-related } \\
\text { common mental disorders: } \\
\text { A one-and three-year } \\
\text { follow-up study. Biomed } \\
\text { Research } \\
\text { International, 2015, 1-7. }\end{array}$} & \multirow[t]{9}{*}{ Mixed/Other } & \multirow[t]{9}{*}{ PWA** } & $\begin{array}{l}\text { Workplace } \\
\text { Mistreatment }\end{array}$ & 223 & .031 \\
\hline & & & Job Demands & 223 & -.083 \\
\hline & & & Job Control & 223 & .032 \\
\hline & & & $\begin{array}{l}\text { Supervisor } \\
\text { Support }\end{array}$ & 223 & .101 \\
\hline & & & $\begin{array}{l}\text { Coworker } \\
\text { Support }\end{array}$ & 223 & .063 \\
\hline & & & Reward & 223 & .044 \\
\hline & & & $\begin{array}{l}\text { Current } \\
\text { Health Status }\end{array}$ & 223 & .304 \\
\hline & & & $\begin{array}{l}\text { Symptoms - } \\
\text { Non Pain } \\
\text { related }\end{array}$ & 223 & -.253 \\
\hline & & & $\begin{array}{l}\text { Absenteeism/ } \\
\text { Sick Leave - } \\
\text { Previous }\end{array}$ & 223 & -.079 \\
\hline \multirow{4}{*}{$\begin{array}{l}\text { Neupane, S., Virtanen, P., } \\
\text { Leino-Arjas, P., Miranda, } \\
\text { H., Siukola, A., \& Nygård, } \\
\text { C. H. (2013). Multi-site } \\
\text { pain and working } \\
\text { conditions as predictors of } \\
\text { work ability in a 4-year } \\
\text { follow-up among food } \\
\text { industry }\end{array}$} & \multirow[t]{4}{*}{ Mixed/Other } & \multirow[t]{4}{*}{ PWA** } & $\begin{array}{l}\text { Physical } \\
\text { demands }\end{array}$ & 734 & -.186 \\
\hline & & & $\begin{array}{l}\text { Environment } \\
\text { al Conditions }\end{array}$ & 734 & -.153 \\
\hline & & & Job Control & 734 & .094 \\
\hline & & & $\begin{array}{l}\text { Supervisor } \\
\text { Support }\end{array}$ & 628 & .158 \\
\hline
\end{tabular}




\begin{tabular}{|c|c|c|c|c|c|}
\hline \multirow{3}{*}{$\begin{array}{l}\text { employees. European } \\
\text { Journal of Pain, 17(3), } \\
444-451 .\end{array}$} & & & $\begin{array}{l}\text { Organization } \\
\text { al Climate }\end{array}$ & 734 & .075 \\
\hline & & & BMI & 734 & -.130 \\
\hline & & & Exercise & 734 & .101 \\
\hline $\begin{array}{l}\text { Neupane, S., Virtanen, P., } \\
\text { Luukkaala, T., Siukola, A., } \\
\& \text { Nygård, C. H. (2014). A } \\
\text { four-year follow-up study } \\
\text { of physical working } \\
\text { conditions and perceived } \\
\text { mental and physical strain } \\
\text { among food industry } \\
\text { workers. Applied } \\
\text { Ergonomics, 45(3), 586- } \\
\text { 591. }\end{array}$ & Blue Collar & PWA* & Age & 248 & .027 \\
\hline \multirow{2}{*}{$\begin{array}{l}\text { Nevanperä, N., Seitsamo, } \\
\text { J., Ala-Mursula, L., Remes, } \\
\text { J., Hopsu, L., Auvinen, J., } \\
\text { \& Laitinen, J. (2016). } \\
\text { Perceived Work Ability in } \\
\text { the Light of Long-Term } \\
\text { and Stress-Related } \\
\text { Unhealthy Behaviors-a } \\
\text { Prospective Cohort } \\
\text { Study. International } \\
\text { Journal of Behavioral } \\
\text { Medicine, 23(2), 179-189. }\end{array}$} & \multirow[t]{2}{*}{ Mixed/Other } & \multirow[t]{2}{*}{ PWA** } & $\begin{array}{l}\text { Job } \\
\text { Resources } \\
\text { (Other) }\end{array}$ & 4429 & .019 \\
\hline & & & BMI & 5654 & .104 \\
\hline \multirow{2}{*}{$\begin{array}{l}\text { Niessen, M. A., Laan, E. } \\
\text { L., Robroek, S. J., Essink- } \\
\text { Bot, M. L., Peek, N., } \\
\text { Kraaijenhagen, R. A., \& } \\
\text { Burdorf, A. (2013). } \\
\text { Determinants of } \\
\text { participation in a web- } \\
\text { based health risk } \\
\text { assessment and } \\
\text { consequences for health } \\
\text { promotion }\end{array}$} & \multirow[t]{2}{*}{ White Collar } & \multirow[t]{2}{*}{ PWA* } & $\begin{array}{l}\text { General } \\
\text { Health }\end{array}$ & 2473 & .290 \\
\hline & & & $\begin{array}{l}\text { Perceived } \\
\text { Stress }\end{array}$ & 2473 & -.280 \\
\hline
\end{tabular}




\begin{tabular}{|c|c|c|c|c|c|}
\hline $\begin{array}{l}\text { programs. Journal of } \\
\text { Medical Internet } \\
\text { Research, 15(8), e151. }\end{array}$ & & & & & \\
\hline \multirow{2}{*}{$\begin{array}{l}\text { Nilsson, S., \& Ekberg, K. } \\
\text { (2013). Employability and } \\
\text { work ability: Returning to } \\
\text { the labour market after } \\
\text { long-term absence. Work: A } \\
\text { journal of Prevention, } \\
\text { Assesment and } \\
\text { Rehabilitation, 44(4), 449- } \\
\text { 457. }\end{array}$} & \multirow[t]{2}{*}{ Mixed/Other } & \multirow[t]{2}{*}{ WAI } & $\begin{array}{l}\text { General } \\
\text { Health }\end{array}$ & 109 & .670 \\
\hline & & & Age & 109 & .260 \\
\hline \multirow{2}{*}{$\begin{array}{l}\text { Nygård, C. H., Eskelinen, } \\
\text { L., Suvanto, S., Tuomi, K., } \\
\text { \& Ilmarinen, J. (1991). } \\
\text { Associations between } \\
\text { functional capacity and } \\
\text { work ability among elderly } \\
\text { municipal employees. } \\
\text { Scandinavian Journal of } \\
\text { Work, Environment \& } \\
\text { Health, 17(1), 122-127. }\end{array}$} & \multirow[t]{2}{*}{ Mixed/other } & \multirow[t]{2}{*}{ WAI } & $\begin{array}{l}\text { Cognitive } \\
\text { Ability }\end{array}$ & 116 & .129 \\
\hline & & & $\begin{array}{l}\text { Physical } \\
\text { Ability }\end{array}$ & 100 & .199 \\
\hline $\begin{array}{l}\text { Oakman, J., Neupane, S., \& } \\
\text { Nygård, C. H. (2016). Does } \\
\text { age matter in predicting } \\
\text { musculoskeletal disorder } \\
\text { risk? An analysis of } \\
\text { workplace predictors over } 4 \\
\text { years. International } \\
\text { Archives of Occupational } \\
\text { and Environmental Health, } \\
\text { 89(7), 1127-1136. }\end{array}$ & Mixed/Other & PWA & Age & 734 & -.080 \\
\hline \multirow{2}{*}{$\begin{array}{l}\text { Oakman, J., \& Wells, Y. } \\
\text { (2016). Working longer: } \\
\text { What is the relationship } \\
\text { between person-- } \\
\text { environment fit and }\end{array}$} & \multirow[t]{2}{*}{ White Collar } & \multirow[t]{2}{*}{ WAI } & Job Demands & 332 & -.150 \\
\hline & & & $\begin{array}{l}\text { Job } \\
\text { Control }\end{array}$ & 332 & .320 \\
\hline
\end{tabular}




\begin{tabular}{|c|c|c|c|c|c|}
\hline \multirow[t]{4}{*}{$\begin{array}{l}\text { retirement intentions? Asia } \\
\text { Pacific Journal of Human } \\
\text { Resources, 54(2), 207-229. }\end{array}$} & & & $\begin{array}{l}\text { Job } \\
\text { Resources } \\
\text { (Other) }\end{array}$ & 332 & .180 \\
\hline & & & Age & 332 & -.070 \\
\hline & & & $\begin{array}{l}\text { Job } \\
\text { Satisfaction }\end{array}$ & 332 & .360 \\
\hline & & & $\begin{array}{l}\text { Retirement } \\
\text { Intentions }\end{array}$ & 332 & -.160 \\
\hline $\begin{array}{l}\text { Oberlinner, C., Yong, M., } \\
\text { Nasterlack, M., Pluto, R. } \\
\text { P., \& Lang, S. (2015). } \\
\text { Combined effect of back } \\
\text { pain and stress on work } \\
\text { ability. Occupational } \\
\text { Medicine, 65(2), 147-153. }\end{array}$ & Mixed/Other & WAI & $\begin{array}{l}\text { Objective } \\
\text { Health } \\
\text { Indicator }\end{array}$ & 653 & .218 \\
\hline \multirow{5}{*}{$\begin{array}{l}\text { Ohta, M., Eguchi, Y., } \\
\text { Inoue, T., Honda, T., } \\
\text { Morita, Y., Konno, Y., \& } \\
\text { Kumashiro, M. (2015). } \\
\text { Effects of bench step } \\
\text { exercise intervention on } \\
\text { work ability in terms of } \\
\text { cardiovascular risk factors } \\
\text { and oxidative stress: a } \\
\text { randomized controlled } \\
\text { study. International } \\
\text { Journal of Occupational } \\
\text { Safety and Ergonomics, } \\
\text { 21(2), 141-149. }\end{array}$} & \multirow[t]{5}{*}{ Blue Collar } & \multirow[t]{5}{*}{ WAI } & $\begin{array}{l}\text { Physical } \\
\text { Health }\end{array}$ & 12 & .741 \\
\hline & & & BMI & 12 & -.520 \\
\hline & & & $\begin{array}{l}\text { Objective } \\
\text { Health } \\
\text { Indicator }\end{array}$ & 12 & .143 \\
\hline & & & $\begin{array}{l}\text { Physical } \\
\text { Ability }\end{array}$ & 12 & .020 \\
\hline & & & Age & 12 & -.050 \\
\hline \multirow{3}{*}{$\begin{array}{l}\text { Ohta, M., Kumashiro, M., } \\
\text { Eguchi, Y., Morita, Y., } \\
\text { Konno, Y., \& Yamato, H. } \\
\text { (2014). The relationship } \\
\text { between work ability and } \\
\text { oxidative stress in Japanese } \\
\text { workers. Ergonomics, } \\
\text { 57(8), 1265-1273. }\end{array}$} & \multirow[t]{3}{*}{ Mixed/Other } & \multirow[t]{3}{*}{ WAI } & $\begin{array}{l}\text { Physical } \\
\text { Health }\end{array}$ & 19 & .451 \\
\hline & & & $\begin{array}{l}\text { Physical } \\
\text { Ability }\end{array}$ & 19 & .220 \\
\hline & & & $\begin{array}{l}\text { Objective } \\
\text { Health } \\
\text { Indicator }\end{array}$ & 19 & .296 \\
\hline
\end{tabular}




\begin{tabular}{|c|c|c|c|c|c|}
\hline & & & BMI & 19 & -.440 \\
\hline & & & Age & 19 & .040 \\
\hline Cadiz et al. (unpublished) & Nurses/Healt & PWA & Job Control & 128 & .323 \\
\hline & & & $\begin{array}{l}\text { Supervisor } \\
\text { Support }\end{array}$ & 128 & .341 \\
\hline & & & $\begin{array}{l}\text { Justice } \\
\text { Perceptions }\end{array}$ & 128 & .225 \\
\hline & & & $\begin{array}{l}\text { Task } \\
\text { Resources }\end{array}$ & 128 & .093 \\
\hline & & & $\begin{array}{l}\text { Core Self } \\
\text { Evaluations }\end{array}$ & 128 & .551 \\
\hline & & & $\begin{array}{l}\text { SOC } \\
\text { Behaviors }\end{array}$ & 128 & .246 \\
\hline & & & $\begin{array}{l}\text { Conscientiou } \\
\text { sness }\end{array}$ & 128 & .360 \\
\hline & & & $\begin{array}{l}\text { Personal } \\
\text { Resource } \\
\text { (Other) }\end{array}$ & 128 & 166 \\
\hline & & & Age & 128 & .024 \\
\hline $\begin{array}{l}\text { Padula, R. S., Comper, M. } \\
\text { L., Moraes, S. A., Sabbagh, }\end{array}$ & Mixed/Other & WAI & $\begin{array}{l}\text { Physical } \\
\text { Ability }\end{array}$ & 37 & .110 \\
\hline $\begin{array}{l}\text { Perracini, M. R. (2013). } \\
\text { fhe work ability index and } \\
\text { functional capacity among }\end{array}$ & & & $\begin{array}{l}\text { Age (both } \\
\text { samples } \\
\text { merged) }\end{array}$ & 79 & -.019 \\
\hline $\begin{array}{l}\text { older workers. Brazilian } \\
\text { Journal of Physical } \\
\text { Therapy, 17(4), 382-391. }\end{array}$ & Mixed/Other & WAI & $\begin{array}{l}\text { Physical } \\
\text { Ability }\end{array}$ & 42 & .014 \\
\hline $\begin{array}{l}\text { Palermo, J., Fuller- } \\
\text { Tyszkiewicz, M., Walker, }\end{array}$ & Mixed/Other & PWA & $\begin{array}{l}\text { Organization } \\
\text { al Climate }\end{array}$ & 306 & .220 \\
\hline $\begin{array}{l}\text { (2013). Primary-and } \\
\text { secondary-level }\end{array}$ & & & $\begin{array}{l}\text { Core Self } \\
\text { Evaluations }\end{array}$ & 306 & .180 \\
\hline organizational predictors of & & & Coping & 306 & .180 \\
\hline
\end{tabular}




\begin{tabular}{|c|c|c|c|c|c|}
\hline \multirow{4}{*}{$\begin{array}{l}\text { work ability. Journal of } \\
\text { Occupational Health } \\
\text { Psychology, 18(2), 220- } \\
229 .\end{array}$} & & & $\begin{array}{l}\text { General } \\
\text { Health }\end{array}$ & 306 & .370 \\
\hline & & & Age & 306 & -.090 \\
\hline & & & $\begin{array}{l}\text { Perceived } \\
\text { Stress }\end{array}$ & 306 & -.330 \\
\hline & & & $\begin{array}{l}\text { Job } \\
\text { Satisfaction }\end{array}$ & 306 & .330 \\
\hline \multirow{2}{*}{$\begin{array}{l}\text { Perkiö-Mäkelä, M. M. } \\
\text { (2000). Finnish farmers' } \\
\text { self-reported morbidity, } \\
\text { work ability, and functional } \\
\text { capacity. Annals of } \\
\text { Agricultural and } \\
\text { Environmental } \\
\text { Medicine, } 7(1), 11-16 .\end{array}$} & \multirow[t]{2}{*}{ Blue Collar } & \multirow[t]{2}{*}{ PWA** } & Depression & 577 & -.377 \\
\hline & & & Age & 438 & -.328 \\
\hline $\begin{array}{l}\text { Phongamwong, C., \& } \\
\text { Deema, H. (2015). The } \\
\text { impact of multi-site } \\
\text { musculoskeletal pain on } \\
\text { work ability among health } \\
\text { care providers. Journal of } \\
\text { Occupational Medicine and } \\
\text { Toxicology, 10(1), 21-26. }\end{array}$ & Mixed/Other & WAI & $\begin{array}{l}\text { Pain/Muscul } \\
\text { oskeletal } \\
\text { Symptoms }\end{array}$ & 254 & -.233 \\
\hline $\begin{array}{l}\text { Pilger, A., Haslacher, H., } \\
\text { Ponocny-Seliger, E., } \\
\text { Perkmann, T., Böhm, K., } \\
\text { Budinsky, A., \& Winker, } \\
\text { R. (2014). Affective and } \\
\text { inflammatory responses } \\
\text { among orchestra musicians } \\
\text { in performance situation. } \\
\text { Brain, Behavior, and } \\
\text { Immunity, 37, 23-29. }\end{array}$ & Mixed/Other & WAI & Age & 48 & -.420 \\
\hline $\begin{array}{l}\text { Pit, S. W., \& Hansen, V. } \\
\text { (2014). Factors influencing } \\
\text { early retirement intentions } \\
\text { in Australian rural general }\end{array}$ & $\begin{array}{l}\text { Nurses/Healt } \\
\text { hcare }\end{array}$ & PWA* & $\begin{array}{l}\text { Retirement } \\
\text { Intentions }\end{array}$ & 91 & .064 \\
\hline
\end{tabular}




\begin{tabular}{|c|c|c|c|c|c|}
\hline $\begin{array}{l}\text { practitioners. Occupational } \\
\text { Medicine, 64(4), 297-304. }\end{array}$ & & & & & \\
\hline $\begin{array}{l}\text { Plat, M. C. J., Frings- } \\
\text { Dresen, M. H., \& Sluiter, J. } \\
\text { K. (2010). Clinimetric } \\
\text { quality of the fire fighting } \\
\text { simulation test as part of } \\
\text { the Dutch fire fighters } \\
\text { Workers' Health } \\
\text { Surveillance. BMC Health } \\
\text { Services Research, 10(1), } \\
\text { 1-9. }\end{array}$ & Mixed/Other & PWA & $\begin{array}{l}\text { Physical } \\
\text { Ability }\end{array}$ & 45 & .274 \\
\hline $\begin{array}{l}\text { Plat, M. C. J., Frings- } \\
\text { Dresen, M. H., \& Sluiter, J. } \\
\text { K. (2012). Impact of } \\
\text { chronic diseases on work } \\
\text { ability in ageing } \\
\text { firefighters. Journal of } \\
\text { Occupational Health, } \\
\text { 54(2), 158-163. }\end{array}$ & Mixed/Other & PWA** & Age & 276 & -.091 \\
\hline $\begin{array}{l}\text { Pohjonen, T. (2001). } \\
\text { Perceived work ability of }\end{array}$ & Blue Collar & PWA** & Age & 636 & -.201 \\
\hline $\begin{array}{l}\text { home care workers in } \\
\text { relation to individual and } \\
\text { work-related factors in } \\
\text { different age groups. } \\
\text { Occupational Medicine, } \\
51(3), 209-217 \text {. }\end{array}$ & Blue Collar & WAI & Age & 636 & -.253 \\
\hline $\begin{array}{l}\text { Pranjić, N., Maleš-Bilić, L., } \\
\text { Beganlić, A., \& } \\
\text { Mustajbegović, J. (2006). } \\
\text { Mobbing, stress, and work } \\
\text { ability index among } \\
\text { physicians in Bosnia and } \\
\text { Herzegovina: survey study. } \\
\text { Croatian Medical Journal, } \\
\text { 47(5), 750-758. }\end{array}$ & $\begin{array}{l}\text { Nurses/Healt } \\
\text { hcare }\end{array}$ & WAI & $\begin{array}{l}\text { Workplace } \\
\text { Mistreatment }\end{array}$ & 511 & -.543 \\
\hline
\end{tabular}




\begin{tabular}{|c|c|c|c|c|c|}
\hline \multirow{4}{*}{$\begin{array}{l}\text { Punakallio, A., Lusa, S., \& } \\
\text { Luukkonen, R. (2004). } \\
\text { Functional, postural and } \\
\text { perceived balance for } \\
\text { predicting the work ability } \\
\text { of } \\
\text { firefighters. International } \\
\text { Archives of Occupational } \\
\text { and Environmental } \\
\text { Health, } 77(7), 482-490 .\end{array}$} & \multirow[t]{4}{*}{ Mixed/Other } & \multirow[t]{2}{*}{ WAI } & $\begin{array}{l}\text { Physical } \\
\text { Ability }\end{array}$ & 81 & .169 \\
\hline & & & $\begin{array}{l}\text { Physical } \\
\text { Health }\end{array}$ & 140 & .642 \\
\hline & & \multirow[t]{2}{*}{ PWA } & $\begin{array}{l}\text { Physical } \\
\text { Ability }\end{array}$ & 81 & .183 \\
\hline & & & $\begin{array}{l}\text { Physical } \\
\text { Health }\end{array}$ & 135 & .551 \\
\hline \multirow{3}{*}{$\begin{array}{l}\text { Ramada, J. M., Serra, C., } \\
\text { Amick III, B. C., Abma, F. } \\
\text { I., Castano, J. R., Pidemunt, } \\
\text { G., \& Delclos, G. L. } \\
\text { (2014). Reliability and } \\
\text { validity of the work role } \\
\text { functioning questionnaire } \\
\text { (Spanish version). Journal } \\
\text { of Occupational } \\
\text { Rehabilitation, 24(4), 640- } \\
649 .\end{array}$} & \multirow[t]{3}{*}{ Mixed/Other } & \multirow[t]{3}{*}{ PWA } & $\begin{array}{l}\text { Physical } \\
\text { Demands }\end{array}$ & 455 & -.577 \\
\hline & & & $\begin{array}{l}\text { Mental/Emot } \\
\text { ional } \\
\text { Demands }\end{array}$ & 455 & -.535 \\
\hline & & & $\begin{array}{l}\text { Quantitative } \\
\text { Job Demands }\end{array}$ & 455 & -.626 \\
\hline $\begin{array}{l}\text { Reeuwijk, K. G., Robroek, } \\
\text { S. J., Niessen, M. A., } \\
\text { Kraaijenhagen, R. A., } \\
\text { Vergouwe, Y., \& Burdorf, } \\
\text { A. (2015). The prognostic } \\
\text { value of the work ability } \\
\text { index for sickness absence } \\
\text { among office workers. PloS } \\
\text { one, } 10(5), \text { e } 0126969 .\end{array}$ & $\begin{array}{l}\text { Mentally } \\
\text { Demanding }\end{array}$ & WAI & $\begin{array}{l}\text { Future } \\
\text { Absenteeism }\end{array}$ & 1331 & -.042 \\
\hline \multirow{2}{*}{$\begin{array}{l}\text { Riedel, N., Müller, A., \& } \\
\text { Ebener, M. (2015). } \\
\text { Applying Strategies of } \\
\text { Selection, Optimization, } \\
\text { and Compensation to } \\
\text { Maintain Work Ability_-A }\end{array}$} & \multirow[t]{2}{*}{ Mixed/Other } & \multirow[t]{2}{*}{ PWA** } & $\begin{array}{l}\text { Quantitative } \\
\text { Job Demands }\end{array}$ & 6057 & -.224 \\
\hline & & & Job Control & 6057 & .182 \\
\hline
\end{tabular}




\begin{tabular}{|c|c|c|c|c|c|}
\hline $\begin{array}{l}\text { Psychosocial Resource } \\
\text { Complementing the Job } \\
\text { Demand-Control Model? } \\
\text { Results From the } \\
\text { Representative lidA Cohort } \\
\text { Study on Work, Age, and } \\
\text { Health in Germany. } \\
\text { Journal of Occupational } \\
\text { and Environmental } \\
\text { Medicine, 57(5), 552-561. }\end{array}$ & & & $\begin{array}{l}\text { SOC } \\
\text { Behaviors }\end{array}$ & 6057 & .083 \\
\hline \multirow{4}{*}{$\begin{array}{l}\text { Riethmeister, V., Brouwer, } \\
\text { S., van der Klink, J., \& } \\
\text { Bültmann, U. (2015). } \\
\text { Work, eat and sleep: } \\
\text { towards a healthy ageing at } \\
\text { work program } \\
\text { offshore. BMC Public } \\
\text { Health, } 16(1), 134-145 .\end{array}$} & \multirow[t]{4}{*}{ Blue Collar } & \multirow[t]{4}{*}{ PWA** } & $\begin{array}{l}\text { General } \\
\text { Health }\end{array}$ & 253 & .480 \\
\hline & & & BMI & 249 & -.120 \\
\hline & & & Age & 255 & -.170 \\
\hline & & & Fatigue & 244 & -.150 \\
\hline \multirow{6}{*}{$\begin{array}{l}\text { Rineer, J. R. } \\
\text { (2016). Supporting the } \\
\text { aging workforce: The } \\
\text { impact of psychosocial } \\
\text { workplace characteristics } \\
\text { on employees work } \\
\text { ability (Order No. } \\
\text { AAI3687643). Available } \\
\text { from PsycINFO. } \\
\text { (1805782207; 2016-17339- } \\
\text { 057). Retrieved from } \\
\text { http://stats.lib.pdx.edu/prox } \\
\text { y.php?url=http://search.pro } \\
\text { quest.com.proxy.lib.pdx.ed } \\
\underline{\text { u/docview/1805782207?acc }} \\
\text { ountid=13265 }\end{array}$} & \multirow[t]{6}{*}{ Blue Collar } & \multirow[t]{6}{*}{ PWA } & $\begin{array}{l}\text { Supervisor } \\
\text { Support }\end{array}$ & 274 & .110 \\
\hline & & & $\begin{array}{l}\text { Coworker } \\
\text { Support }\end{array}$ & 274 & .290 \\
\hline & & & $\begin{array}{l}\text { Justice } \\
\text { Perceptions }\end{array}$ & 274 & .025 \\
\hline & & & $\begin{array}{l}\text { Organization } \\
\text { al Climate }\end{array}$ & 274 & .210 \\
\hline & & & $\begin{array}{l}\text { General } \\
\text { Health }\end{array}$ & 274 & .410 \\
\hline & & & Age & 274 & -.200 \\
\hline $\begin{array}{l}\text { Roelen, C. A., Heymans, } \\
\text { M. W., Twisk, J. W., van } \\
\text { der Klink, J. J., Groothoff, } \\
\text { J. W., \& van Rhenen, W. } \\
\text { (2014). Work Ability Index }\end{array}$ & Blue Collar & WAI & Age & 9530 & -.202 \\
\hline
\end{tabular}




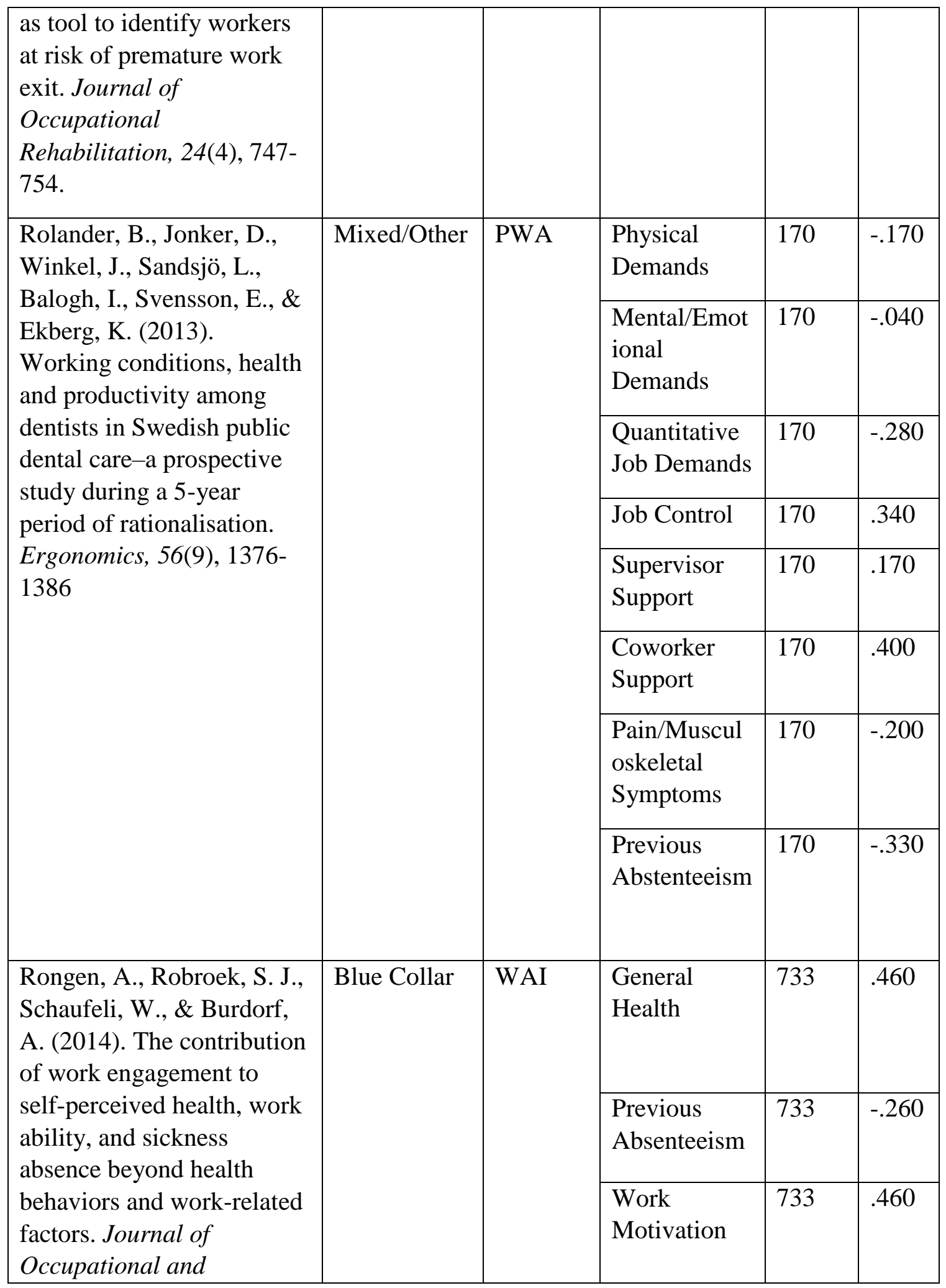




\begin{tabular}{|c|c|c|c|c|c|}
\hline $\begin{array}{l}\text { Environmental } \\
\text { Medicine, 56(8), 892-897. }\end{array}$ & & & & & \\
\hline $\begin{array}{l}\text { Rongen, A., Robroek, S. J., } \\
\text { Heijden, B. I., Schouteten, } \\
\text { R., Hasselhorn, H. M., \& } \\
\text { Burdorf, A. (2014). } \\
\text { Influence of work-related } \\
\text { characteristics and work } \\
\text { ability on changing } \\
\text { employer or leaving the } \\
\text { profession among nursing } \\
\text { staff. Journal of Nursing } \\
\text { Management, 22(8), 1065- } \\
\text { 1075. }\end{array}$ & WAI & $\begin{array}{l}\text { Nursing/ } \\
\text { Healthca } \\
\text { re }\end{array}$ & $\begin{array}{l}\text { Exit } \\
\text { Behaviors }\end{array}$ & 9,927 & -.114 \\
\hline $\begin{array}{l}\text { Rostamabadi, A., } \\
\text { Zamanian, Z., \& Sedaghat, } \\
\text { Z. (2017). Factors } \\
\text { associated with work } \\
\text { ability index (WAI) among } \\
\text { intensive care units'(ICUs') } \\
\text { nurses. Journal of } \\
\text { Occupational } \\
\text { Health, 59(2), 147-155. }\end{array}$ & $\begin{array}{l}\text { Nurses/Healt } \\
\text { hcare }\end{array}$ & WAI & $\begin{array}{l}\text { Current } \\
\text { Health Status }\end{array}$ & 214 & .538 \\
\hline \multirow{4}{*}{$\begin{array}{l}\text { Rostamabadi, A., } \\
\text { Mazloumi, A., \& } \\
\text { Foroushani, A. R. (2014). } \\
\text { Work Ability Index (WAI) } \\
\text { and its health-related } \\
\text { determinants among Iranian } \\
\text { farmers working in small } \\
\text { farm enterprises. Journal of } \\
\text { Occupational } \\
\text { Health, 56(6), 478-484. }\end{array}$} & \multirow[t]{4}{*}{ Blue Collar } & \multirow[t]{4}{*}{ WAI } & Sleep & 294 & .298 \\
\hline & & & BMI & 294 & -.061 \\
\hline & & & Smoking & 294 & -.108 \\
\hline & & & Age & 294 & -.544 \\
\hline \multirow{3}{*}{$\begin{array}{l}\text { Ruitenburg, M. M., Frings- } \\
\text { Dresen, M. H., \& Sluiter, J. } \\
\text { K. (2012). The prevalence } \\
\text { of common mental } \\
\text { disorders among hospital }\end{array}$} & \multirow[t]{3}{*}{$\begin{array}{l}\text { Nurses/Healt } \\
\text { hcare }\end{array}$} & \multirow[t]{3}{*}{ PWA** } & $\begin{array}{l}\text { Current } \\
\text { Health Status }\end{array}$ & 405 & .429 \\
\hline & & & Depression & 405 & -.548 \\
\hline & & & Anxiety & 405 & -.475 \\
\hline
\end{tabular}




\begin{tabular}{|c|c|c|c|c|c|}
\hline \multirow{4}{*}{$\begin{array}{l}\text { physicians and their } \\
\text { association with self- } \\
\text { reported work ability: a } \\
\text { cross-sectional study. BMC } \\
\text { Health Services } \\
\text { Research, } 12(1), 1-7 .\end{array}$} & & & Age & 405 & .000 \\
\hline & & & Burnout & 389 & -.527 \\
\hline & & & Fatigue & 389 & -.326 \\
\hline & & & $\begin{array}{l}\text { Perceived } \\
\text { Stress }\end{array}$ & 389 & -.584 \\
\hline \multirow{5}{*}{$\begin{array}{l}\text { Sahlin, E., Ahlborg, G., } \\
\text { Matuszczyk, J. V., \& } \\
\text { Grahn, P. (2014). Nature- } \\
\text { based stress management } \\
\text { course for individuals at } \\
\text { risk of adverse health } \\
\text { effects from work-related } \\
\text { stress - effects on stress } \\
\text { related symptoms, } \\
\text { workability and sick } \\
\text { leave. International } \\
\text { Journal of Environmental } \\
\text { Research and Public } \\
\text { Health, 11(6), 6586-6611. }\end{array}$} & \multirow[t]{5}{*}{ Mixed/Other } & \multirow[t]{5}{*}{ PWA** } & $\begin{array}{l}\text { Symptoms - } \\
\text { Non Pain } \\
\text { related }\end{array}$ & 31 & -.239 \\
\hline & & & Sleep & 31 & .021 \\
\hline & & & $\begin{array}{l}\text { Previous } \\
\text { Absenteeism }\end{array}$ & 30 & -.620 \\
\hline & & & $\begin{array}{l}\text { Pain/Muscul } \\
\text { oskeletal } \\
\text { Symptoms }\end{array}$ & 31 & -.307 \\
\hline & & & $\begin{array}{l}\text { Objective } \\
\text { Health } \\
\text { Indicator }\end{array}$ & 31 & .198 \\
\hline $\begin{array}{l}\text { Salonen, P., Arola, H., } \\
\text { Nygård, C. H., Huhtala, H., } \\
\text { \& Koivisto, A. M. (2003). } \\
\text { Factors associated with } \\
\text { premature departure from } \\
\text { working life among ageing } \\
\text { food industry } \\
\text { employees. Occupational } \\
\text { Medicine, 53(1), 65-68. }\end{array}$ & Blue Collar & WAI & Retirement & 126 & -.367 \\
\hline \multirow{4}{*}{$\begin{array}{l}\text { Saltychev, M., Laimi, K., } \\
\text { Pentti, J., Kivimäki, M., \& } \\
\text { Vahtera, J. (2014). Short- } \\
\text { and long-term changes in } \\
\text { perceived work ability after } \\
\text { interdisciplinary } \\
\text { rehabilitation of chronic } \\
\text { musculoskeletal disorders: } \\
\text { prospective cohort study }\end{array}$} & \multirow[t]{4}{*}{ Mixed/Other } & \multirow[t]{4}{*}{ PWA** } & Job Control & 548 & .214 \\
\hline & & & $\begin{array}{l}\text { General } \\
\text { Health }\end{array}$ & 765 & .146 \\
\hline & & & $\begin{array}{l}\text { Current } \\
\text { Health Status }\end{array}$ & 854 & .098 \\
\hline & & & $\begin{array}{l}\text { Previous } \\
\text { Absenteeism }\end{array}$ & 854 & -.139 \\
\hline
\end{tabular}




\begin{tabular}{|c|c|c|c|c|c|}
\hline \multirow{7}{*}{$\begin{array}{l}\text { among } 854 \text { rehabilitants. } \\
\text { Clinical Rehabilitation, } \\
\text { 28(6), 592-603. }\end{array}$} & & & $\begin{array}{l}\text { Mental } \\
\text { Health }\end{array}$ & 848 & .266 \\
\hline & & & Anxiety & 561 & -.303 \\
\hline & & & BMI & 841 & -.120 \\
\hline & & & Exercise & 846 & .088 \\
\hline & & & Drinking & 850 & -.006 \\
\hline & & & Smoking & 825 & -.072 \\
\hline & & & $\begin{array}{l}\text { Turnover } \\
\text { Intentions }\end{array}$ & 831 & -.238 \\
\hline \multirow{3}{*}{$\begin{array}{l}\text { Sampaio, R. F., Coelho, C. } \\
\text { M., Barbosa, F. B., } \\
\text { Mancini, M. C., \& Parreira, } \\
\text { V. F. (2009). Work ability } \\
\text { and stress in a bus } \\
\text { transportation company in } \\
\text { Belo Horizonte, } \\
\text { Brazil. Ciência \& Saúde } \\
\text { Coletiva, 14(1), 287-296. }\end{array}$} & \multirow[t]{3}{*}{ Blue Collar } & \multirow[t]{3}{*}{ WAI } & $\begin{array}{l}\text { Quantitative } \\
\text { Job Demands }\end{array}$ & 108 & -.334 \\
\hline & & & Age & 108 & -.158 \\
\hline & & & Job Control & 108 & .034 \\
\hline $\begin{array}{l}\text { Sauni, R., Toivio, P., } \\
\text { Pääkkönen, R., Malmström, } \\
\text { J., \& Uitti, J. (2015). Work } \\
\text { disability after diagnosis of } \\
\text { hand-arm vibration } \\
\text { syndrome. International } \\
\text { Archives of Occupational } \\
\text { and Environmental } \\
\text { Health, 88(8), 1061-1068. }\end{array}$ & Blue Collar & PWA** & $\begin{array}{l}\text { Current } \\
\text { Health Status }\end{array}$ & 102 & .379 \\
\hline $\begin{array}{l}\text { Schouten, L. S., Joling, C. } \\
\text { I., van der Gulden, J. W., } \\
\text { Heymans, M. W., } \\
\text { Bültmann, U., \& Roelen, C. } \\
\text { A. (2015). Screening } \\
\text { manual and office workers } \\
\text { for risk of long-term } \\
\text { sickness absence: cut-off } \\
\text { points for the Work Ability }\end{array}$ & Mixed/Other & WAI & $\begin{array}{l}\text { Future } \\
\text { Absenteeism }\end{array}$ & 981 & -.065 \\
\hline
\end{tabular}




\begin{tabular}{|c|c|c|c|c|c|}
\hline $\begin{array}{l}\text { Index. Scandinavian } \\
\text { Journal of Work, } \\
\text { Environment \& Health, } \\
\text { 41(1), 36-42. }\end{array}$ & & & & & \\
\hline \multirow{6}{*}{$\begin{array}{l}\text { Schulz, H., Zacher, H., \& } \\
\text { Lippke, S. (2017). The } \\
\text { importance of team health } \\
\text { climate for health-related } \\
\text { outcomes of white-collar } \\
\text { workers. Frontiers in } \\
\text { Psychology, 8(74),1-14. }\end{array}$} & \multirow[t]{6}{*}{ White Collar } & \multirow[t]{6}{*}{ PWA } & Job Demands & 6449 & -.470 \\
\hline & & & Job Control & 6449 & .240 \\
\hline & & & $\begin{array}{l}\text { General } \\
\text { Health }\end{array}$ & 6449 & .640 \\
\hline & & & $\begin{array}{l}\text { Symptoms - } \\
\text { Non Pain } \\
\text { related }\end{array}$ & 6449 & -.560 \\
\hline & & & Depression & 6449 & -.530 \\
\hline & & & Age & 6449 & -.300 \\
\hline \multirow{7}{*}{$\begin{array}{l}\text { Seibt, R., Spitzer, S., } \\
\text { Blank, M., \& Scheuch, K. } \\
\text { (2009). Predictors of work } \\
\text { ability in occupations with } \\
\text { psychological stress. } \\
\text { Journal of Public Health, } \\
\text { 17(1), 9-18. }\end{array}$} & \multirow[t]{7}{*}{ White Collar } & \multirow[t]{7}{*}{ WAI } & $\begin{array}{l}\text { Justice } \\
\text { Perceptions }\end{array}$ & 160 & .330 \\
\hline & & & $\begin{array}{l}\text { Cognitive/M } \\
\text { ental Ability }\end{array}$ & 160 & .180 \\
\hline & & & $\begin{array}{l}\text { General } \\
\text { Health }\end{array}$ & 160 & .540 \\
\hline & & & $\begin{array}{l}\text { Physical } \\
\text { Health }\end{array}$ & 160 & .155 \\
\hline & & & $\begin{array}{l}\text { Physical } \\
\text { Ability }\end{array}$ & 160 & .210 \\
\hline & & & BMI & 160 & -.160 \\
\hline & & & Burnout & 160 & -.540 \\
\hline \multirow{2}{*}{$\begin{array}{l}\text { Sell, L., Lund, H. L., } \\
\text { Holtermann, A., \& } \\
\text { Søgaard, K. (2014). The } \\
\text { interactions between pain, } \\
\text { pain-related fear of } \\
\text { movement and } \\
\text { productivity. Occupational } \\
\text { Medicine, 64(5), 376-381. }\end{array}$} & \multirow[t]{2}{*}{$\begin{array}{l}\text { Blue } \\
\text { ColNlar }\end{array}$} & \multirow[t]{2}{*}{ WAI } & $\begin{array}{l}\text { Pain/Muscul } \\
\text { oskeletal } \\
\text { Symptoms }\end{array}$ & 309 & -.447 \\
\hline & & & $\begin{array}{l}\text { Job } \\
\text { Performance }\end{array}$ & 309 & .240 \\
\hline
\end{tabular}




\begin{tabular}{|c|c|c|c|c|c|}
\hline $\begin{array}{l}\text { Shiri, R., Kaila-Kangas, L., } \\
\text { Ahola, K., Kivekäs, T., } \\
\text { Viikari-Juntura, E., } \\
\text { Heliövaara, M., \& Leino- } \\
\text { Arjas, P. (2013). The } \\
\text { relation of co-occurring } \\
\text { musculoskeletal pain and } \\
\text { depressive symptoms with } \\
\text { work ability. Journal of } \\
\text { Occupational and } \\
\text { Environmental Medicine, } \\
\text { 55(11), 1281-1285. }\end{array}$ & Mixed/Other & PWA & $\begin{array}{l}\text { Retirement } \\
\text { Intentions }\end{array}$ & 4,009 & -.185 \\
\hline \multirow{6}{*}{$\begin{array}{l}\text { Sluiter, J. K., \& Frings- } \\
\text { Dresen, M. H. (2008). } \\
\text { Quality of life and illness } \\
\text { perception in working and } \\
\text { sick-listed chronic RSI } \\
\text { patients. International } \\
\text { Archives of Occupational } \\
\text { and Environmental } \\
\text { Health, 81(4), 495-501. }\end{array}$} & \multirow[t]{6}{*}{ Mixed/Other } & \multirow[t]{6}{*}{ PWA** } & $\begin{array}{l}\text { General } \\
\text { Health }\end{array}$ & 1121 & .610 \\
\hline & & & $\begin{array}{l}\text { Current } \\
\text { Health Status }\end{array}$ & 1121 & .618 \\
\hline & & & $\begin{array}{l}\text { Mental } \\
\text { Health }\end{array}$ & 1121 & .301 \\
\hline & & & $\begin{array}{l}\text { Physical } \\
\text { Health }\end{array}$ & 1121 & .571 \\
\hline & & & $\begin{array}{l}\text { Pain/Muscul } \\
\text { oskeletal } \\
\text { Symptoms }\end{array}$ & 1121 & -.411 \\
\hline & & & Fatigue & 1121 & -.400 \\
\hline \multirow{2}{*}{$\begin{array}{l}\text { Smolander, J., Sörensen, } \\
\text { L., Pekkonen, M., \& Alén, } \\
\text { M. (2009). Muscle } \\
\text { performance, work ability } \\
\text { and physical functioning in } \\
\text { middle-aged } \\
\text { men. Occupational } \\
\text { Medicine, 60(1), 78-80.† } \\
\text { Merged with Sorensen et } \\
\text { al., } 2007 \text { (Same sample) }\end{array}$} & \multirow[t]{2}{*}{ Mixed/Other } & \multirow[t]{2}{*}{ WAI } & $\begin{array}{l}\text { Physical } \\
\text { Ability }\end{array}$ & 43 & .228 \\
\hline & & & $\begin{array}{l}\text { Physical } \\
\text { Health }\end{array}$ & 51 & .630 \\
\hline $\begin{array}{l}\text { Sörensen, L., Smolander, } \\
\text { J., Louhevaara, V., }\end{array}$ & Mixed/Other & WAI & $\begin{array}{l}\text { Physical } \\
\text { Ability }\end{array}$ & 89 & .164 \\
\hline
\end{tabular}




\begin{tabular}{|c|c|c|c|c|c|}
\hline $\begin{array}{l}\text { Korhonen, O., \& Oja, P. } \\
\text { (2000). Physical activity, } \\
\text { fitness and body } \\
\text { composition of Finnish } \\
\text { police officers: a } 15 \text {-year } \\
\text { follow-up } \\
\text { study. Occupational } \\
\text { Medicine, 50(1), 3-10. }\end{array}$ & & & $\begin{array}{l}\text { Physical } \\
\text { Activity }\end{array}$ & 89 & -.060 \\
\hline \multirow{7}{*}{$\begin{array}{l}\text { Sörensen, L. E., Pekkonen, } \\
\text { M. M., Männikkö, K. H., } \\
\text { Louhevaara, V. A., } \\
\text { Smolander, J., \& Alén, M. } \\
\text { J. (2008). Associations } \\
\text { between work ability, } \\
\text { health-related quality of } \\
\text { life, physical activity and } \\
\text { fitness among middle-aged } \\
\text { men. Applied Ergonomics, } \\
\text { 39(6), 786-791. }\end{array}$} & \multirow[t]{7}{*}{ Blue Collar } & \multirow[t]{7}{*}{ WAI } & $\begin{array}{l}\text { General } \\
\text { Health }\end{array}$ & 196 & .580 \\
\hline & & & $\begin{array}{l}\text { Mental } \\
\text { Health }\end{array}$ & 196 & .471 \\
\hline & & & $\begin{array}{l}\text { Pain/Muscul } \\
\text { oskeletal } \\
\text { Symptoms }\end{array}$ & 196 & -.520 \\
\hline & & & $\begin{array}{l}\text { Physical } \\
\text { Ability }\end{array}$ & 196 & .100 \\
\hline & & & $\begin{array}{l}\text { Physical } \\
\text { Health }\end{array}$ & 196 & .530 \\
\hline & & & Exercise & 196 & .000 \\
\hline & & & Fatigue & 196 & -.650 \\
\hline $\begin{array}{l}\text { Sörensen, L., Honkalehto, } \\
\text { S., Kallinen, M., Pekkonen, } \\
\text { M., Louhevaara, V., } \\
\text { Smolander, J., \& Alén, M. } \\
\text { (2007). Are } \\
\text { cardiorespiratory fitness } \\
\text { and walking performance } \\
\text { associated with self- } \\
\text { reported quality of life and } \\
\text { work ability?. International } \\
\text { Journal of Occupational } \\
\text { Medicine and } \\
\text { Environmental Health, } \\
\text { 20(3), 257-264. }\end{array}$ & $\begin{array}{l}\text { See } \\
\text { Smolander et } \\
\text { al., } 2009\end{array}$ & & & & \\
\hline
\end{tabular}




\begin{tabular}{|c|c|c|c|c|c|}
\hline \multirow{3}{*}{$\begin{array}{l}\text { Sormunen, E., Remes, J., } \\
\text { Hassi, J., Pienimaki, T., \& } \\
\text { Rintamaki, H., (2009). } \\
\text { Factors associated with } \\
\text { self-estimated work ability } \\
\text { and musculoskeletal } \\
\text { symptoms among male and } \\
\text { female workers in cooled } \\
\text { food-processing } \\
\text { facilities. Industrial } \\
\text { Health, 47(3), 271-282. }\end{array}$} & \multirow[t]{3}{*}{ Blue Collar } & \multirow[t]{3}{*}{$\mathrm{PWA}^{* *}$} & $\begin{array}{l}\text { Previous } \\
\text { Absenteeism }\end{array}$ & 1117 & -.341 \\
\hline & & & $\begin{array}{l}\text { Pain/Muscul } \\
\text { oskeletal } \\
\text { Symptoms }\end{array}$ & 1117 & .295 \\
\hline & & & Exercise & 1117 & .162 \\
\hline \multirow{2}{*}{$\begin{array}{l}\text { Sugimura, H., \& Thériault, } \\
\text { G. (2010). Impact of } \\
\text { supervisor support on work } \\
\text { ability in an IT } \\
\text { company. Occupational } \\
\text { Medicine, 60(6), 451-457. }\end{array}$} & White Collar & PWA* & $\begin{array}{l}\text { Supervisor } \\
\text { Support }\end{array}$ & 1157 & .228 \\
\hline & White Collar & WAI & $\begin{array}{l}\text { Supervisor } \\
\text { Support }\end{array}$ & 1157 & .310 \\
\hline $\begin{array}{l}\text { Stordeur, S., \& D'Hoore, } \\
\text { W. (2007). Organizational } \\
\text { configuration of hospitals } \\
\text { succeeding in attracting and } \\
\text { retaining nurses. Journal of } \\
\text { Advanced Nursing, 57(1), } \\
45-58 .\end{array}$ & $\begin{array}{l}\text { Nursing/Hea } \\
\text { lthcare }\end{array}$ & WAI & Turnover & 1175 & -.266 \\
\hline $\begin{array}{l}\text { Sun, J., Buys, N., \& Wang, } \\
\text { X. (2013). Depression in } \\
\text { employees in privately } \\
\text { owned enterprises in China: } \\
\text { is it related to work } \\
\text { environment and work } \\
\text { ability?. International } \\
\text { Journal of Environmental } \\
\text { Research and Public } \\
\text { Health, 10(4), 1152-1167. }\end{array}$ & Mixed/Other & PWA* & Depression & 4800 & -.244 \\
\hline $\begin{array}{l}\text { Szlachta, E., Gawlik- } \\
\text { Chmiel, B., \& Kallus, K. } \\
\text { W. (2012). Do the long- } \\
\text { term unemployed regard } \\
\text { themselves as able to }\end{array}$ & Mixed/Other & PWA** & Age & 530 & -.343 \\
\hline
\end{tabular}




\begin{tabular}{|c|c|c|c|c|c|}
\hline $\begin{array}{l}\text { work?. Journal of Public } \\
\text { Health, 20(5), 505-511. }\end{array}$ & & & & & \\
\hline \multirow{3}{*}{$\begin{array}{l}\text { Tavakoli-Fard, N., } \\
\text { Mortazavi, S. A., } \\
\text { Kuhpayehzadeh, J., \& } \\
\text { Nojomi, M. (2016). Quality } \\
\text { of life, Work ability and } \\
\text { other important indicators } \\
\text { of women's occupational } \\
\text { health. International } \\
\text { Journal of Occupational } \\
\text { Medicine and } \\
\text { Environmental } \\
\text { Health, 29(1), 77-84. }\end{array}$} & \multirow[t]{3}{*}{ Blue Collar } & \multirow[t]{3}{*}{ WAI } & $\begin{array}{l}\text { Mental } \\
\text { Health }\end{array}$ & 328 & .369 \\
\hline & & & $\begin{array}{l}\text { Physical } \\
\text { Health }\end{array}$ & 328 & .422 \\
\hline & & & Age & 328 & -.029 \\
\hline \multirow{6}{*}{$\begin{array}{l}\text { Torp, S., Nielsen, R. A., } \\
\text { Gudbergsson, S. B., \& } \\
\text { Dahl, A. A. (2012). } \\
\text { Worksite adjustments and } \\
\text { work ability among } \\
\text { employed cancer } \\
\text { survivors. Supportive Care } \\
\text { in Cancer, 20(9), 2149- } \\
2156 .\end{array}$} & \multirow[t]{6}{*}{ Mixed/Other } & \multirow[t]{6}{*}{ PWA** } & $\begin{array}{l}\text { Quantitative } \\
\text { Job Demands }\end{array}$ & 653 & -.140 \\
\hline & & & Job Control & 653 & .080 \\
\hline & & & $\begin{array}{l}\text { Supervisor } \\
\text { Support }\end{array}$ & 653 & .090 \\
\hline & & & $\begin{array}{l}\text { Coworker } \\
\text { Support }\end{array}$ & 653 & .150 \\
\hline & & & $\begin{array}{l}\text { Current } \\
\text { Health Status }\end{array}$ & 653 & .225 \\
\hline & & & Age & 653 & .000 \\
\hline $\begin{array}{l}\text { Tuomi, K., Huuhtanen, P., } \\
\text { Nykyri, E., \& Ilmarinen, J. } \\
\text { (2001). Promotion of work } \\
\text { ability, the quality of work } \\
\text { and } \\
\text { retirement. Occupational } \\
\text { Medicine, 51(5), 318-324. }\end{array}$ & Mixed/Other & WAI & $\begin{array}{l}\text { Job } \\
\text { Performance }\end{array}$ & 1016 & .169 \\
\hline $\begin{array}{l}\text { van de Vijfeijke, H., } \\
\text { Leijten, F. R., Ybema, J. F., } \\
\text { van den Heuvel, S. G., }\end{array}$ & Mixed/Other & PWA** & Coping & 8824 & .075 \\
\hline
\end{tabular}




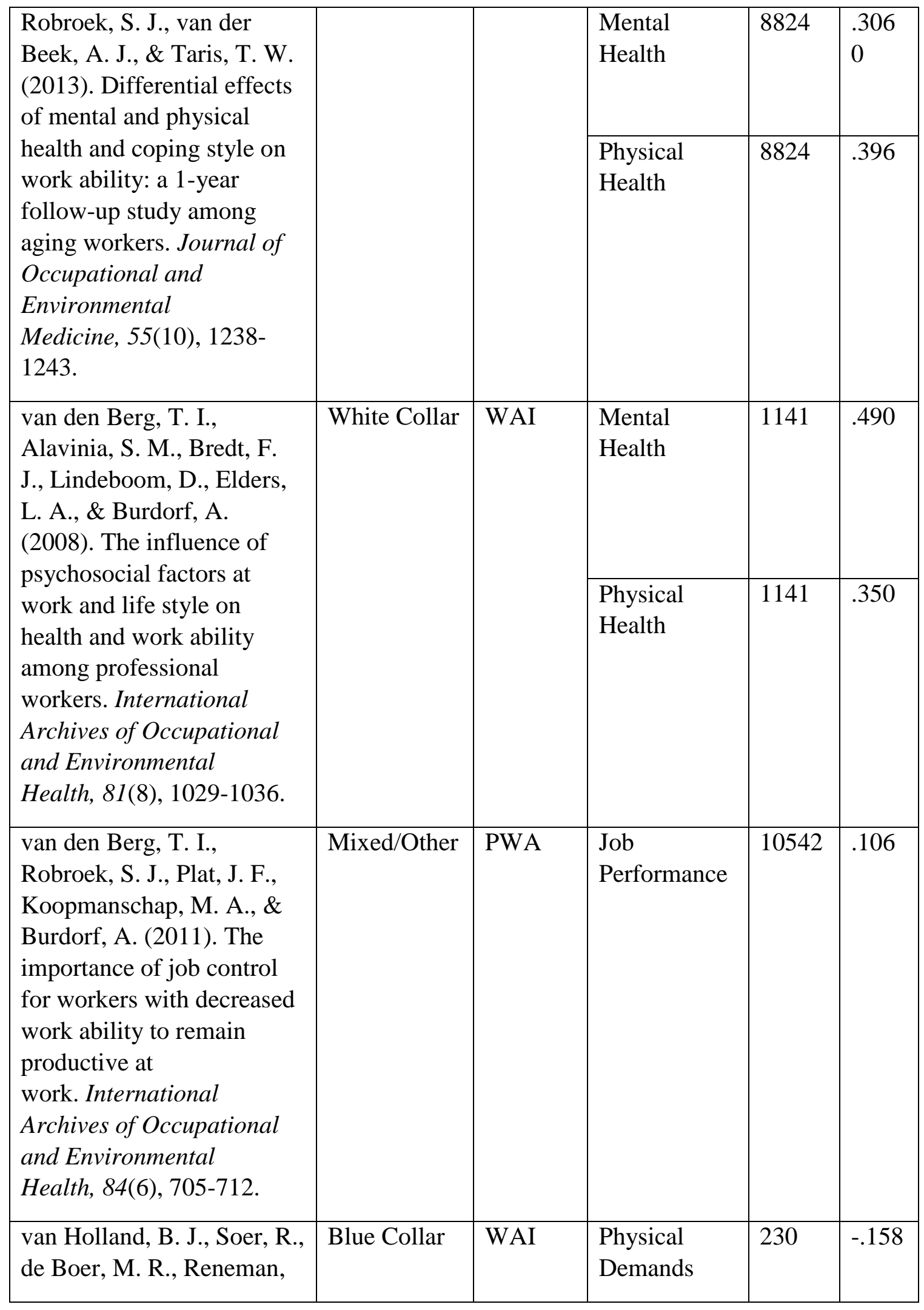




\begin{tabular}{|c|c|c|c|c|c|}
\hline \multirow{9}{*}{$\begin{array}{l}\text { M. F., \& Brouwer, S. } \\
\text { (2015). Workers' health } \\
\text { surveillance in the meat } \\
\text { processing industry: work } \\
\text { and health indicators } \\
\text { associated with work } \\
\text { ability. Journal of } \\
\text { Occupational } \\
\text { Rehabilitation, 25(3), 618- } \\
\text { 626. }\end{array}$} & & & $\begin{array}{l}\text { Mental/Emot } \\
\text { ional } \\
\text { Demands }\end{array}$ & 230 & -.163 \\
\hline & & & $\begin{array}{l}\text { Objective } \\
\text { Health } \\
\text { Indicator }\end{array}$ & 230 & -.006 \\
\hline & & & $\begin{array}{l}\text { Physical } \\
\text { Health }\end{array}$ & 230 & -.006 \\
\hline & & & $\begin{array}{l}\text { Physical } \\
\text { Ability }\end{array}$ & 230 & .203 \\
\hline & & & Smoking & 230 & -.016 \\
\hline & & & Drinking & 230 & -.120 \\
\hline & & & $\begin{array}{l}\text { Health } \\
\text { Behaviors }\end{array}$ & 230 & .023 \\
\hline & & & Age & 230 & -.014 \\
\hline & & & Fatigue & 230 & -.153 \\
\hline $\begin{array}{l}\text { van Muijen, P., Duijts, S. } \\
\text { F., Bonefaas-Groenewoud, } \\
\text { K., van der Beek, A. J., \& } \\
\text { Anema, J. R. (2014). } \\
\text { Factors associated with } \\
\text { work disability in } \\
\text { employed cancer survivors } \\
\text { at 24-month sick } \\
\text { leave. BMC Cancer, 14(1), } \\
\text { 236-246. }\end{array}$ & Mixed/Other & PWA* & $\begin{array}{l}\text { Future } \\
\text { Disability } \\
\text { Status }\end{array}$ & 253 & -.332 \\
\hline \multirow{4}{*}{$\begin{array}{l}\text { Vasconcelos, S. P., Fischer, } \\
\text { F. M., Reis, A. O. A., \& } \\
\text { Moreno, C. R. D. C. } \\
\text { (2011). Factors associated } \\
\text { with work ability and } \\
\text { perception of fatigue } \\
\text { among nursing personnel } \\
\text { from Amazonia. Revista } \\
\text { Brasileira de }\end{array}$} & \multirow{4}{*}{$\begin{array}{l}\text { Nurses/Healt } \\
\text { hcare }\end{array}$} & \multirow[t]{4}{*}{ WAI } & Job Demands & 271 & -.157 \\
\hline & & & $\begin{array}{l}\text { General } \\
\text { Health }\end{array}$ & 272 & .196 \\
\hline & & & Fatigue & 272 & -.467 \\
\hline & & & Age & 272 & -.057 \\
\hline
\end{tabular}




\begin{tabular}{|c|c|c|c|c|c|}
\hline $\begin{array}{l}\text { Epidemiologia, 14(4), 688- } \\
697 .\end{array}$ & & & & & \\
\hline \multirow{4}{*}{$\begin{array}{l}\text { Vastamäki, J., Moser, K., \& } \\
\text { Paul, K. I. (2009). How } \\
\text { stable is sense of } \\
\text { coherence? Changes } \\
\text { following an intervention } \\
\text { for unemployed } \\
\text { individuals. Scandinavian } \\
\text { Journal of Psychology, } \\
\text { 50(2), 161-171. }\end{array}$} & \multirow[t]{4}{*}{ Mixed/Other } & \multirow[t]{4}{*}{ PWA* } & $\begin{array}{l}\text { Positive } \\
\text { Affect }\end{array}$ & 74 & .280 \\
\hline & & & $\begin{array}{l}\text { Current } \\
\text { Health Status }\end{array}$ & 69 & .280 \\
\hline & & & Age & 74 & -.040 \\
\hline & & & $\begin{array}{l}\text { Perceived } \\
\text { Stress }\end{array}$ & 74 & -.370 \\
\hline \multirow{2}{*}{$\begin{array}{l}\text { Vastamäki, J., Wolff, H. } \\
\text { G., Paul, K. I., \& Moser, K. } \\
\text { (2014). Sense of Coherence } \\
\text { Mediates the Effects of } \\
\text { Low Work Ability on } \\
\text { Mental Distress During } \\
\text { Unemployment. Journal of } \\
\text { Workplace Behavioral } \\
\text { Health, 29(4), 317-332. }\end{array}$} & \multirow[t]{2}{*}{ Mixed/Other } & \multirow[t]{2}{*}{ WAI } & $\begin{array}{l}\text { Mental } \\
\text { Health }\end{array}$ & 98 & .508 \\
\hline & & & Age & 98 & .300 \\
\hline \multirow{2}{*}{$\begin{array}{l}\text { Vedovato, T. G., \& } \\
\text { Monteiro, I. (2014). Health } \\
\text { conditions and factors } \\
\text { related to the work ability } \\
\text { of teachers. Industrial } \\
\text { Health, 52(2), 121-128. }\end{array}$} & \multirow[t]{2}{*}{ White Collar } & \multirow[t]{2}{*}{ WAI } & $\begin{array}{l}\text { General } \\
\text { Health }\end{array}$ & 153 & .542 \\
\hline & & & Age & 258 & .011 \\
\hline \multirow{4}{*}{$\begin{array}{l}\text { Verhoef, J. A., Miedema, } \\
\text { H. S., Bramsen, I., \& } \\
\text { Roebroeck, M. E. (2012). } \\
\text { Using the work limitations } \\
\text { questionnaire in patients } \\
\text { with a chronic condition in } \\
\text { the Netherlands. Journal of } \\
\text { Occupational and } \\
\text { Environmental } \\
\text { Medicine, 54(10), 1293- } \\
1299 .\end{array}$} & \multirow[t]{4}{*}{ Mixed/Other } & \multirow[t]{4}{*}{ WAI } & $\begin{array}{l}\text { Physical } \\
\text { Demands }\end{array}$ & 125 & -.280 \\
\hline & & & $\begin{array}{l}\text { Mental/Emot } \\
\text { ional } \\
\text { Demands }\end{array}$ & 125 & -.640 \\
\hline & & & $\begin{array}{l}\text { Quantitative } \\
\text { Job Demands }\end{array}$ & 125 & -.650 \\
\hline & & & Job Demands & 125 & -.725 \\
\hline
\end{tabular}




\begin{tabular}{|c|c|c|c|c|c|}
\hline $\begin{array}{l}\text { Vindholmen, S., Høigaard, } \\
\text { R., Espnes, G. A., \& Seiler, } \\
\text { S. (2014). Return to work } \\
\text { after vocational } \\
\text { rehabilitation: does } \\
\text { mindfulness matter?. } \\
\text { Psychology Research and } \\
\text { Behavior Management, 7, } \\
77-88 .\end{array}$ & Mixed/Other & PWA & $\begin{array}{l}\text { Personal } \\
\text { Resources } \\
\text { (Other) }\end{array}$ & 80 & .190 \\
\hline \multirow{9}{*}{$\begin{array}{l}\text { Viotti, S., Guidetti, G., } \\
\text { Loera, B., Martini, M., } \\
\text { Sottimano, I., \& Converso, } \\
\text { D. (2017). Stress, work } \\
\text { ability, and an aging } \\
\text { workforce: A study among } \\
\text { women aged } 50 \text { and } \\
\text { over. International Journal } \\
\text { of Stress Management, 24, } \\
98-121 .\end{array}$} & \multirow[t]{9}{*}{ White Collar } & \multirow[t]{9}{*}{ PWA } & $\begin{array}{l}\text { Physical } \\
\text { Demands }\end{array}$ & 202 & -.290 \\
\hline & & & $\begin{array}{l}\text { Mental/Emot } \\
\text { ional } \\
\text { Demands }\end{array}$ & 202 & -.216 \\
\hline & & & Job Demands & 202 & -.380 \\
\hline & & & Job Control & 202 & .340 \\
\hline & & & $\begin{array}{l}\text { Supervisor } \\
\text { Support }\end{array}$ & 202 & .260 \\
\hline & & & $\begin{array}{l}\text { Coworker } \\
\text { Support }\end{array}$ & 202 & .250 \\
\hline & & & $\begin{array}{l}\text { Task } \\
\text { Resources }\end{array}$ & 202 & .361 \\
\hline & & & Age & 202 & -.090 \\
\hline & & & Burnout & 202 & -.630 \\
\hline \multirow{5}{*}{$\begin{array}{l}\text { von Bonsdorff, M. B., } \\
\text { Seitsamo, J., Ilmarinen, J., } \\
\text { Nygård, C. H., von } \\
\text { Bonsdorff, M. E., \& } \\
\text { Rantanen, T. (2011). Work } \\
\text { ability in midlife as a } \\
\text { predictor of mortality and } \\
\text { disability in later life: a 28- } \\
\text { year prospective follow-up } \\
\text { study. Canadian Medical }\end{array}$} & \multirow[t]{5}{*}{ Blue Collar } & \multirow[t]{5}{*}{ PWA** } & $\begin{array}{l}\text { Current } \\
\text { Health Status }\end{array}$ & 944 & .420 \\
\hline & & & $\begin{array}{l}\text { Pain/Muscul } \\
\text { oskeletal } \\
\text { Symptoms }\end{array}$ & 944 & -.468 \\
\hline & & & BMI & 944 & -.079 \\
\hline & & & Exercise & 944 & .211 \\
\hline & & & Drinking & 944 & -.106 \\
\hline
\end{tabular}




\begin{tabular}{|c|c|c|c|c|c|}
\hline Association Journal, & & & Smoking & 577 & -.147 \\
\hline $\begin{array}{l}105(4), 25 J-242 . \\
(\mathrm{FLAMF}) \div\end{array}$ & & & Age & 944 & -.132 \\
\hline & & & $\begin{array}{l}\text { Future } \\
\text { Disability } \\
\text { Status }\end{array}$ & 307 & -.214 \\
\hline & Blue Collar & PWA** & $\begin{array}{l}\text { Current } \\
\text { Health Status }\end{array}$ & 816 & .285 \\
\hline & & & $\begin{array}{l}\text { Pain/Muscul } \\
\text { oskeletal } \\
\text { Symptoms }\end{array}$ & 816 & -.489 \\
\hline & & & BMI & 816 & -.048 \\
\hline & & & Exercise & 816 & .192 \\
\hline & & & Drinking & 816 & .193 \\
\hline & & & Smoking & 739 & -.061 \\
\hline & & & Age & 816 & -.137 \\
\hline & & & $\begin{array}{l}\text { Future } \\
\text { Disability } \\
\text { Status }\end{array}$ & 407 & -.294 \\
\hline & White Collar & PWA** & $\begin{array}{l}\text { Current } \\
\text { Health Status }\end{array}$ & 388 & .431 \\
\hline & & & $\begin{array}{l}\text { Pain/Muscul } \\
\text { oskeletal } \\
\text { Symptoms }\end{array}$ & 388 & -.476 \\
\hline & & & BMI & 388 & -.112 \\
\hline & & & Exercise & 388 & .253 \\
\hline & & & Drinking & 388 & -.133 \\
\hline & & & Smoking & 225 & -.330 \\
\hline & & & Age & 388 & -.215 \\
\hline
\end{tabular}




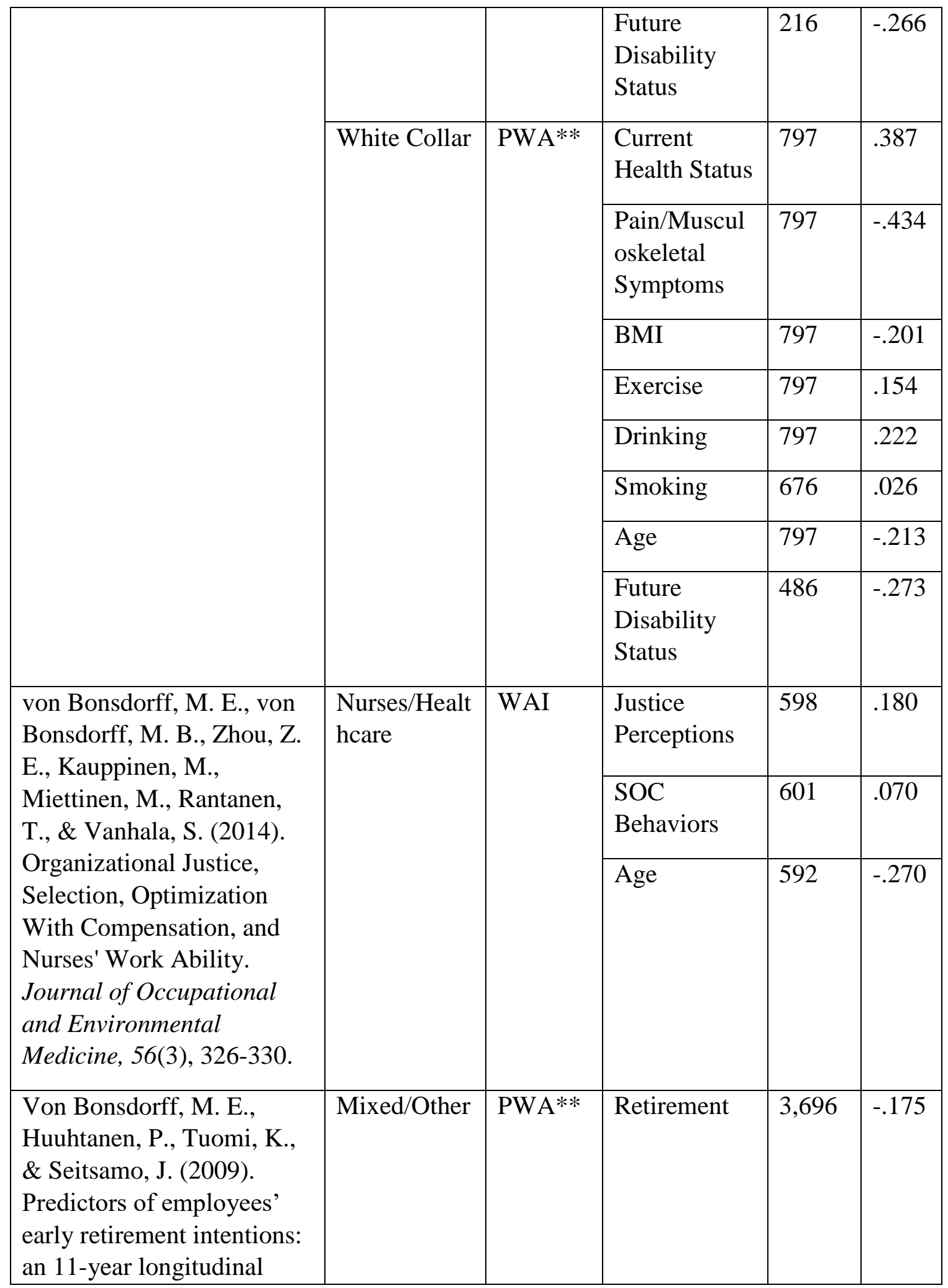




\begin{tabular}{|c|c|c|c|c|c|}
\hline $\begin{array}{l}\text { study. Occupational } \\
\text { Medicine, } 60(2), 94-100 .\end{array}$ & & & & & \\
\hline $\begin{array}{l}\text { von Bonsdorff, M. E., } \\
\text { Vanhala, S., Seitsamo, J., } \\
\text { Janhonen, M., \& Husman, } \\
\text { P. (2010). Employee well- } \\
\text { being, early-retirement } \\
\text { intentions, and company } \\
\text { performance. Journal of } \\
\text { Occupational and } \\
\text { Environmental } \\
\text { Medicine, 52(12), 1255- } \\
\text { 1261. }\end{array}$ & Mixed/Other & WAI & $\begin{array}{l}\text { Retirement } \\
\text { Intentions }\end{array}$ & 457 & -.345 \\
\hline \multirow{8}{*}{$\begin{array}{l}\text { Wagenaar, A. F., Kompier, } \\
\text { M. A., Houtman, I. L., van } \\
\text { den Bossche, S. N., \& } \\
\text { Taris, T. W. (2015). Who } \\
\text { gets fired, who gets re- } \\
\text { hired: the role of workers' } \\
\text { contract, age, health, work } \\
\text { ability, performance, work } \\
\text { satisfaction and employee } \\
\text { investments. International } \\
\text { Archives of Occupational } \\
\text { and Environmental } \\
\text { Health, 88(3), 321-334. }\end{array}$} & \multirow[t]{8}{*}{ Mixed/Other } & \multirow[t]{8}{*}{ PWA } & Reward & 2407 & .085 \\
\hline & & & $\begin{array}{l}\text { Job } \\
\text { Resources } \\
\text { (Other) }\end{array}$ & 2407 & .065 \\
\hline & & & $\begin{array}{l}\text { General } \\
\text { Health }\end{array}$ & 2644 & .265 \\
\hline & & & $\begin{array}{l}\text { Previous } \\
\text { Absenteeism }\end{array}$ & 2644 & -.085 \\
\hline & & & $\begin{array}{l}\text { Job } \\
\text { Performance }\end{array}$ & 2,407 & .232 \\
\hline & & & Age & 2644 & -.045 \\
\hline & & & Burnout & 2,407 & -.320 \\
\hline & & & $\begin{array}{l}\text { Job } \\
\text { Satisfaction }\end{array}$ & 2,407 & .225 \\
\hline $\begin{array}{l}\text { WåHlin-Norgren, C., } \\
\text { Ekberg, K., \& Öberg, B. } \\
\text { (2011). Is an expert } \\
\text { diagnosis enough for } \\
\text { assessment of sick leave for }\end{array}$ & Mixed/Other & WAI & $\begin{array}{l}\text { Current } \\
\text { Health Status }\end{array}$ & 207 & .635 \\
\hline
\end{tabular}




\begin{tabular}{|c|c|c|c|c|c|}
\hline $\begin{array}{l}\text { employees with } \\
\text { musculoskeletal and mental } \\
\text { disorders?. Disability and } \\
\text { Rehabilitation, 33(13-14), } \\
\text { 1147-1156. }\end{array}$ & & & & & \\
\hline \multirow{2}{*}{$\begin{array}{l}\text { Walker, E. J., Jackson, C. } \\
\text { A., Egan, H. H., \& Tonkin, } \\
\text { M. (2015). Workability and } \\
\text { mental wellbeing among } \\
\text { therapeutic prison } \\
\text { officers. Occupational } \\
\text { Medicine, 65(7), 549-551. }\end{array}$} & \multirow[t]{2}{*}{ Mixed/Other } & \multirow[t]{2}{*}{ PWA } & Sleep & 41 & .526 \\
\hline & & & $\begin{array}{l}\text { Mental } \\
\text { Health }\end{array}$ & 41 & .467 \\
\hline $\begin{array}{l}\text { Walsh, I. A., Oishi, J., \& } \\
\text { Coury, H. J. (2008). } \\
\text { Clinical and functional } \\
\text { aspects of work-related } \\
\text { musculoskeletal disorders } \\
\text { among active } \\
\text { workers. Revista de Saude } \\
\text { Publica, 42(1), 108-116. }\end{array}$ & Blue Collar & WAI & $\begin{array}{l}\text { Physical } \\
\text { Health }\end{array}$ & 134 & .705 \\
\hline \multirow{3}{*}{$\begin{array}{l}\text { Weigl, M., Mueller, A., } \\
\text { Hornung, S., Zacher, H., \& } \\
\text { Angerer, P. (2013). The } \\
\text { moderating effects of job } \\
\text { control and selection, } \\
\text { optimization, and } \\
\text { compensation strategies on } \\
\text { the age-work ability } \\
\text { relationship. Journal of } \\
\text { Organizational Behavior, } \\
\text { 34(5), 607-628. }\end{array}$} & \multirow[t]{3}{*}{$\begin{array}{l}\text { Nurses/Healt } \\
\text { hcare }\end{array}$} & \multirow[t]{3}{*}{ PWA } & Job Control & 173 & .200 \\
\hline & & & $\begin{array}{l}\text { SOC } \\
\text { Behaviors }\end{array}$ & 173 & .050 \\
\hline & & & Age & 173 & -.140 \\
\hline \multirow{2}{*}{$\begin{array}{l}\text { Weng, L. C., Huang, H. L., } \\
\text { Wang, Y. W., Lee, W. C., } \\
\text { Chen, K. H., \& Yang, T. Y. } \\
\text { (2014). The effect of self- } \\
\text { efficacy, depression and } \\
\text { symptom distress on } \\
\text { employment status and }\end{array}$} & \multirow[t]{2}{*}{ Mixed/Other } & \multirow[t]{2}{*}{ PWA } & $\begin{array}{l}\text { Core Self } \\
\text { Evaluations }\end{array}$ & 106 & .490 \\
\hline & & & $\begin{array}{l}\text { Symptoms - } \\
\text { Non Pain } \\
\text { related }\end{array}$ & 106 & -.340 \\
\hline
\end{tabular}




\begin{tabular}{|c|c|c|c|c|c|}
\hline $\begin{array}{l}\text { leisure activities of liver } \\
\text { transplant recipients. } \\
\text { Journal of Advanced } \\
\text { Nursing, } 70(7), 1573-1583 \text {. }\end{array}$ & & & Depression & 106 & -.340 \\
\hline $\begin{array}{l}\text { Wilke, C., Ashton, P., Elis, } \\
\text { T., Biallas, B., \& Froböse, } \\
\text { I. (2015). Analysis of work } \\
\text { ability and work-related } \\
\text { physical activity of } \\
\text { employees in a medium- } \\
\text { sized business. BMC } \\
\text { Research Notes, } 8(1), 803- \\
808 \text {. }\end{array}$ & Mixed/Other & WAI & Age & 148 & -.120 \\
\hline \multirow{5}{*}{$\begin{array}{l}\text { Yong, M., Nasterlack, M., } \\
\text { Pluto, R. P., Elmerich, K., } \\
\text { Karl, D., \& Knauth, P. } \\
\text { (2010). Is health, measured } \\
\text { by work ability index, } \\
\text { affected by 12-hour } \\
\text { rotating shift } \\
\text { schedules?. Chronobiology } \\
\text { International, 27(5), 1135- } \\
\text { 1148. }\end{array}$} & \multirow[t]{5}{*}{ Mixed/Other } & \multirow[t]{5}{*}{ WAI } & BMI & 924 & -062 \\
\hline & & & Exercise & 824 & .035 \\
\hline & & & Smoking & 666 & -.086 \\
\hline & & & Drinking & 917 & .048 \\
\hline & & & Age & 924 & -.078 \\
\hline \multirow[t]{7}{*}{ Zacher et al. (unpublished) } & \multirow[t]{7}{*}{ Mixed/Other } & \multirow[t]{7}{*}{ PWA } & $\begin{array}{l}\text { Core Self } \\
\text { Evaluations }\end{array}$ & 316 & .520 \\
\hline & & & $\begin{array}{l}\text { SOC } \\
\text { Behaviors }\end{array}$ & 316 & .181 \\
\hline & & & $\begin{array}{l}\text { Physical } \\
\text { Health }\end{array}$ & 316 & .480 \\
\hline & & & Age & 316 & .150 \\
\hline & & & Burnout & 316 & -.420 \\
\hline & & & Motivation & 316 & .370 \\
\hline & & & $\begin{array}{l}\text { Job } \\
\text { Performance }\end{array}$ & 316 & .480 \\
\hline
\end{tabular}


Note. $\dagger$ Indicates the sample from this study was used in multiple studies. Correlates from these samples were cross-checked to be sure that the sample and correlate were not duplicated in the study database. *Perceived work ability was measured using both the single item "current WA compared to lifetime best" and another perceived work ability item. **Indicates perceived WA was only assessed via the single item, "current WA compared to lifetime best." 